Portland State University

PDXScholar

Summer 1-1-2012

\title{
Short-Term Plasticity at the Schaffer Collateral: A \\ New Model with Implications for Hippocampal \\ Processing
}

Andrew Hamilton Toland

Portland State University

Follow this and additional works at: https://pdxscholar.library.pdx.edu/open_access_etds

Part of the Musculoskeletal, Neural, and Ocular Physiology Commons, Neurology Commons, and the Other Mathematics Commons

Let us know how access to this document benefits you.

\section{Recommended Citation}

Toland, Andrew Hamilton, "Short-Term Plasticity at the Schaffer Collateral: A New Model with Implications for Hippocampal Processing" (2012). Dissertations and Theses. Paper 756.

https://doi.org/10.15760/etd.756

This Dissertation is brought to you for free and open access. It has been accepted for inclusion in Dissertations and Theses by an authorized administrator of PDXScholar. Please contact us if we can make this document more accessible: pdxscholar@pdx.edu. 


\title{
Short-Term Plasticity at the Schaffer Collateral: A New Model with Implications for Hippocampal Processing
}

$$
\text { by }
$$

Andrew Hamilton Toland

A dissertation submitted in partial fulfillment of the requirements for the degree of

\author{
Doctor of Philosophy \\ in \\ Systems Science
}

Dissertation Committee:

George G. Lendaris, Chair

Patrick Roberts

Mathew Frerking

Dacian Daescu

Gerardo Lafferriere

Portland State University

(C) 2012 


\begin{abstract}
A new mathematical model of short-term synaptic plasticity (STP) at the Schaffer collateral is introduced. Like other models of STP, the new model relates short-term synaptic plasticity to an interaction between facilitative and depressive dynamic influences. Unlike previous models, the new model successfully simulates facilitative and depressive dynamics within the framework of the synaptic vesicle cycle. The novelty of the model lies in the description of a competitive interaction between calciumsensitive proteins for binding sites on the vesicle release machinery.

By attributing specific molecular causes to observable presynaptic effects, the new model of STP can predict the effects of specific alterations to the presynaptic neurotrans mitter release mechanism. This understanding will guide further experiments into presynaptic functionality, and may contribute insights into the development of pharmaceuticals that target illnesses manifesting aberrant synaptic dynamics, such as Fragile$\mathrm{X}$ syndrome and schizophrenia.

The new model of STP will also add realism to brain circuit models that simulate cognitive processes such as attention and memory. The hippocampal processing loop is an example of a brain circuit involved in memory formation. The hippocampus filters and organizes large amounts of spatio-temporal data in real time according to contextual significance. The role of synaptic dynamics in the hippocampal system is specu-
\end{abstract}


lated to help keep the system close to a region of instability that increases encoding capacity and discriminating capability. In particular, synaptic dynamics at the Schaffer collateral are proposed to coordinate the output of the highly dynamic CA3 region of the hippocampus with the phase-code in the CA1 that modulates communication between the hippocampus and the neo-cortex. 


\section{Dedication}

This dissertation is dedicated to my wife, Rebecca, and children, Wes and Fiona. 


\section{Acknowledgements}

I would like to express gratitude to my advisors and mentors over the years during which I pursued the doctoral degree. First, to George Lendaris for introducing me to neural networks and computational intelligence. George's pursuit of excellence has motivated me, and many others, to overcome our own expectations and realize our potential. I am honored to be one of George's last students. During my time in George's lab, I was fortunate to meet a number of extraordinary people. Among them, Roberto Santiago, whose analysis of fixed-weight adaptive neural networks inspired the system-level model of the hippocampus in this dissertation. Roberto introduced me to Pat Roberts, who later became my mentor in computational neuroscience, and helped me to appreciate the dynamical behavior and computational power of single neurons. Pat championed my eclectic skill set and convinced Matt Frerking that I would be a good candidate to work on his synaptic dynamics project. Both Pat and Matt are world-class neuroscientists (computational and experimental, repectively), as well as exceptional human beings, and as such, have helped me to relax, consider all things in perspective, and above all maintain professionalism and intellectual honesty. In Matt's lab I had the great opportunity to work with a handful of remarkable people who will remain lifelong inspirations, especially Emily Johnson, who introduced me to the concept of mental schema.

The flexibility of the Systems Science graduate program at Portland State University 
allowed me to develop an interdisciplinary curriculum that encompassed mathematics, engineering, and biology coursework. The professors from whom I took classes at PSU were encouraging at every step, and will remain for me examples of excellence in teaching and analytical thinking. In particular, I will always refer to the professionalism and rigor of professors Dacian Daescu and Gerardo Lafferriere in the math department, James McNames in the engineering department, and Randy Zelick in the biology department. The Systems Science professors, including George Lendaris, Martin Zwick and Wayne Wakeland, constitute a delightful blend of pragmatic and holistic thinking.

There are also a number of professionals in the broader Portland community without whom this dissertation may not have materialized. In particular, I want to recognize my primary care physician, Max Tenscher, who, through investigative work, diagnosed my unusual blood condition, and probably saved my life, as well as this dissertation. Also, thanks goes to Robert Tatsumi, who surgically reconstructed my lower back, allowing me to sit comfortably again for the first time in about fifteen years.

My mother and father deserve special mention here as well. Because of them, I have enjoyed opportunities to indulge my intellectual curiosity, and the fortune of meeting intellectuals from the artistic, scientific, engineering, religious, and medical professions. When I was 14, my mother encouraged and supported my interest in designing loudspeakers. This interest has subsequently provided a remarkably constant reference for acoustical, electro-mechanical, and psycho-physical phenomena. Loudspeakers 
encompass a surprising amount of intellectual territory, and provide a tactile example for many concepts that apply to signal-processing and filter theory. Furthermore, my father's involvement in the mineralogical community provided numerous opportunities to visit places and people, and experience the display of nature's creativity in the myriad forms and colors of geological formations and crystallized minerals. The sense of wonder for the magnitude and scale of natural phenomenon I owe to my father.

Finally, I don't know how to express my gratitude to my wife for tirelessly supporting me through the dark and the bright times. Without her I may have given up hope on any number of occasions. 


\section{Contents}

$\begin{array}{lr}\text { Abstract } & \text { i } \\ \text { Dedication } & \text { ii } \\ \text { Acknowledgements } & \text { iv } \\ \text { List of Tables } & \mathbf{x i} \\ \text { List of Figures } & \mathbf{x i i} \\ \text { Preface } & \mathbf{x v} \\ \text { Chapter 1. Neural Systems } & 1\end{array}$

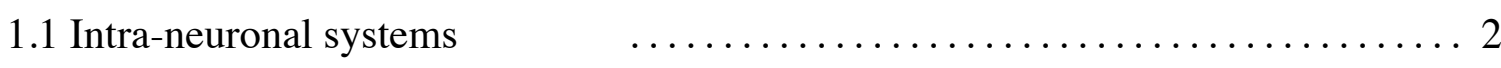

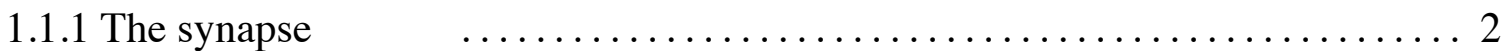

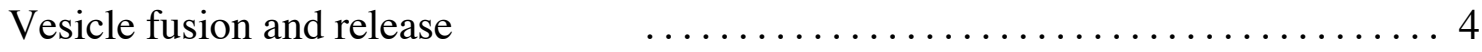

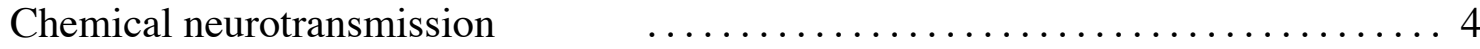

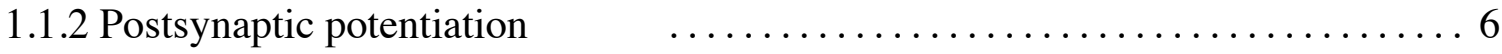

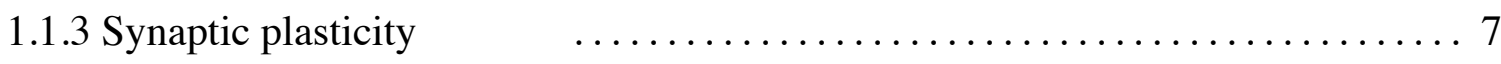

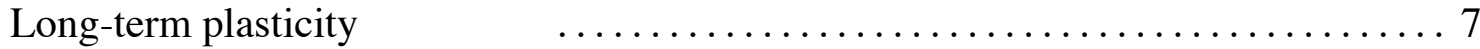

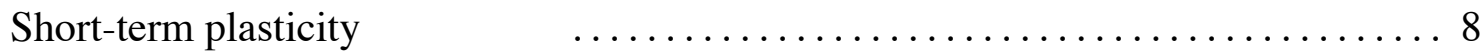

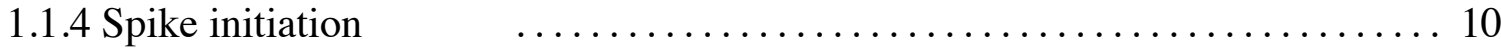

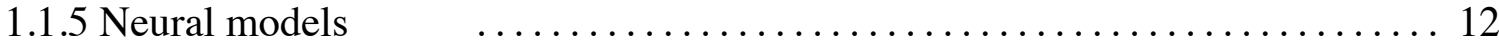

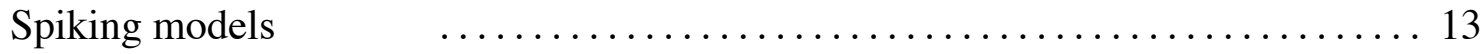

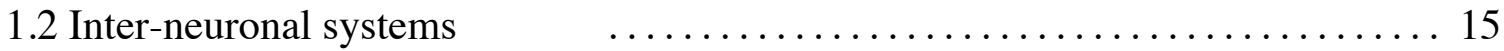

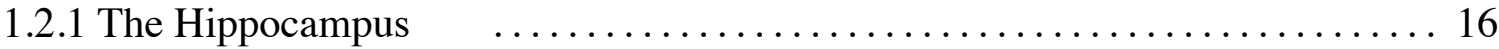

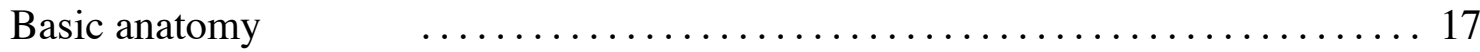

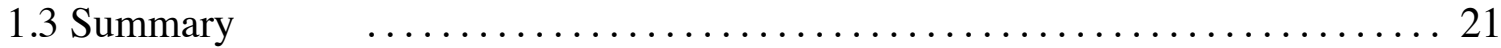

Chapter 2. Modeling STP at the Schaffer collateral 22

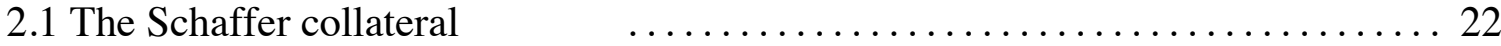

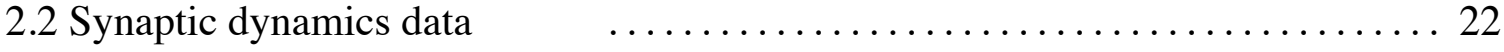

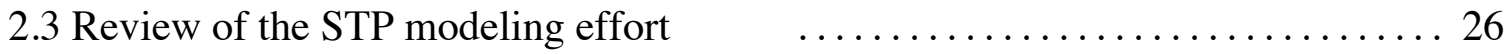

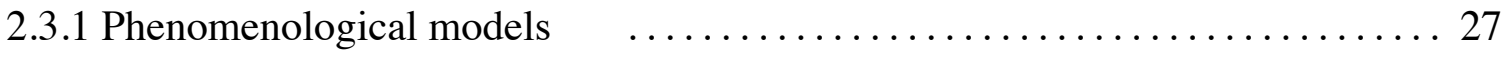

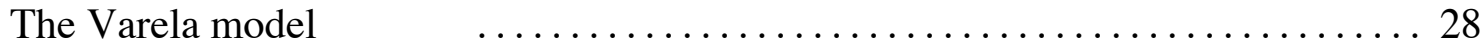




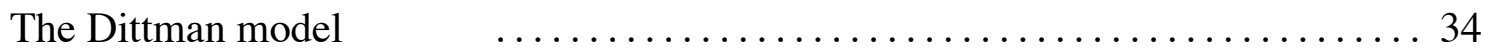

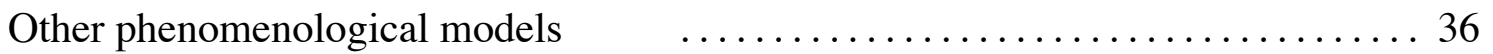

Summary of the phenomenological modeling approach $\quad \ldots \ldots \ldots \ldots \ldots \ldots .41$

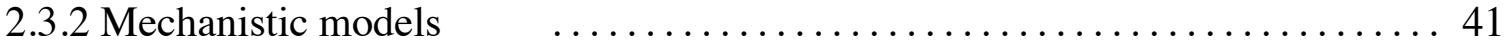

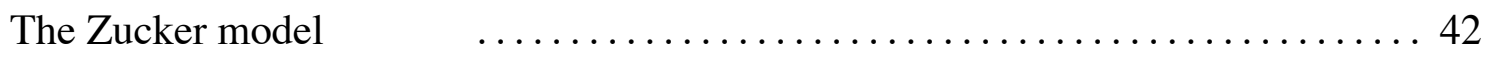

Summary of the mechanistic modeling approach $\quad \ldots \ldots \ldots \ldots \ldots \ldots \ldots 43$

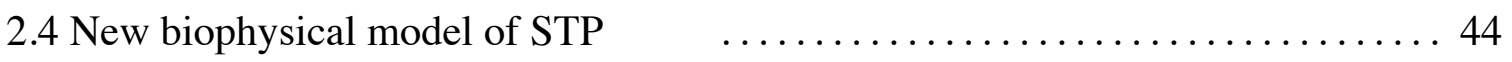

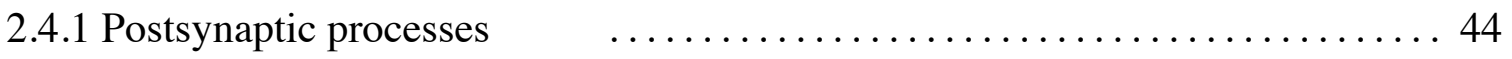

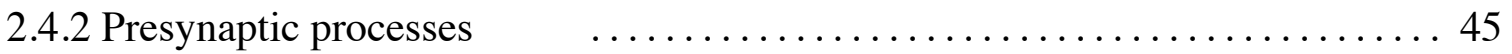

2.4.3 Release rates, vesicle pools, and calcium sensors $\quad \ldots \ldots \ldots \ldots \ldots \ldots .47$

2.4.4 Extracellular calcium influx and decay $\quad \ldots \ldots \ldots \ldots \ldots \ldots \ldots \ldots \ldots \ldots \ldots \ldots \ldots$

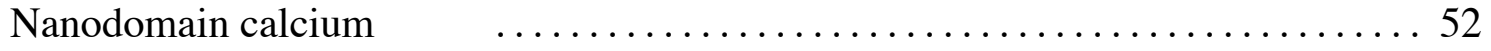

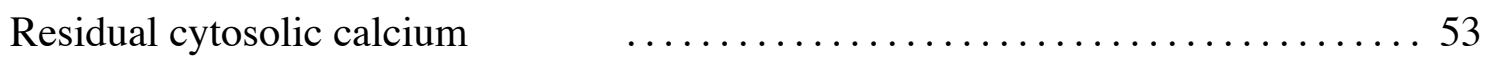

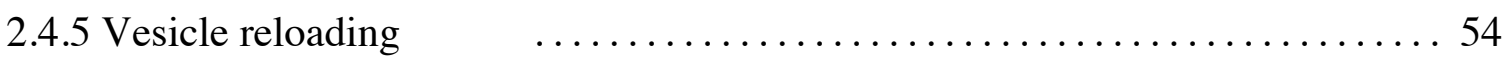

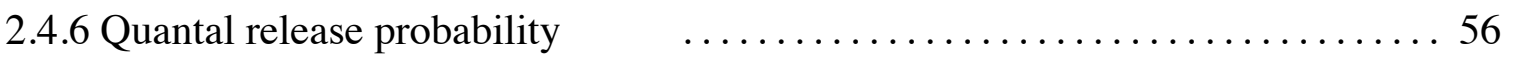

2.4.7 Determination of vesicle release energy $\quad \ldots \ldots \ldots \ldots \ldots \ldots \ldots \ldots \ldots$

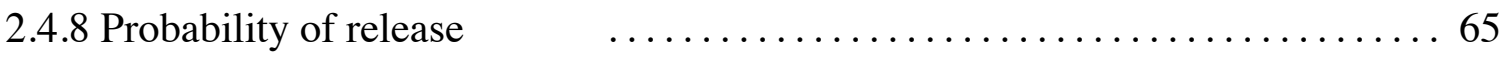

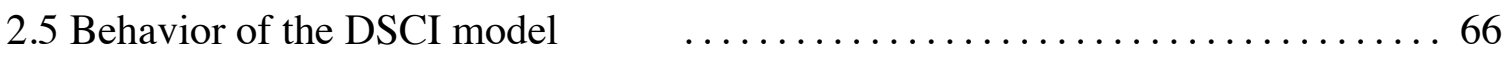

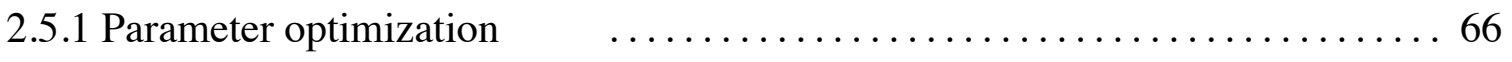

2.5.2 The DSCI model explains the Frerking data $\quad \ldots \ldots \ldots \ldots \ldots \ldots \ldots \ldots \ldots \ldots \ldots \ldots \ldots$

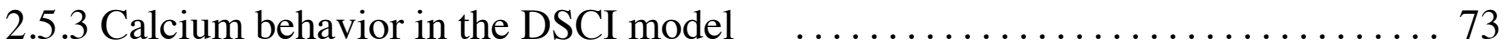

Calcium dependency of release probability $\quad \ldots \ldots \ldots \ldots \ldots \ldots \ldots \ldots \ldots$

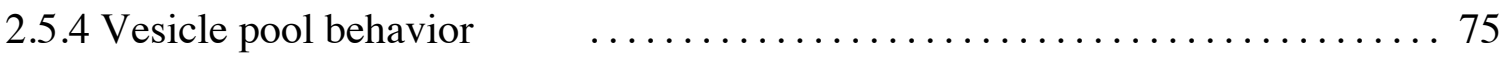

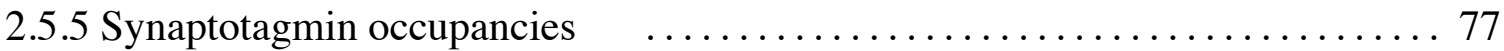

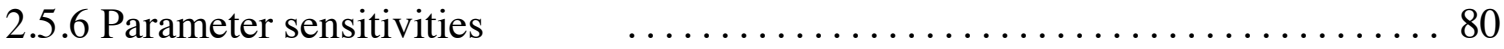

2.5.7 Pharmacological verification (potential) $\quad \ldots \ldots \ldots \ldots \ldots \ldots \ldots \ldots \ldots \ldots$

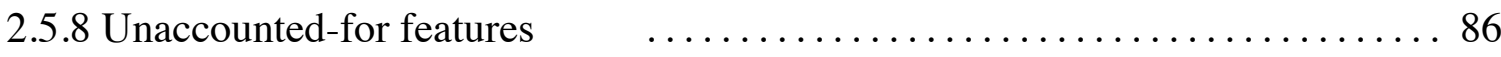

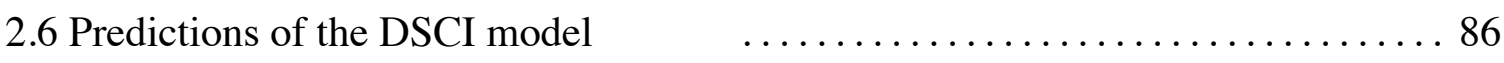

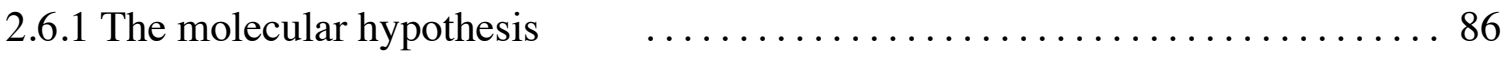

2.6.2 The distinction between vesicle pools $\quad \ldots \ldots \ldots \ldots \ldots \ldots \ldots \ldots \ldots$

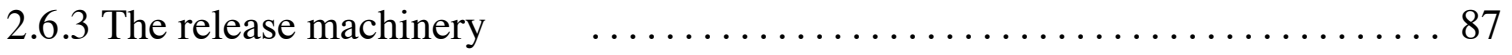

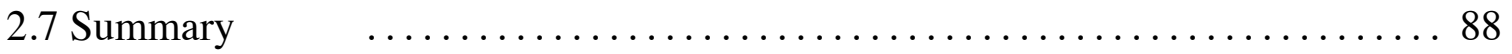

Chapter 3. Characterization of STP at the Schaffer collateral 89 
3.1 Preliminary considerations $\quad \ldots \ldots \ldots \ldots \ldots \ldots \ldots \ldots \ldots \ldots \ldots . \ldots \ldots$

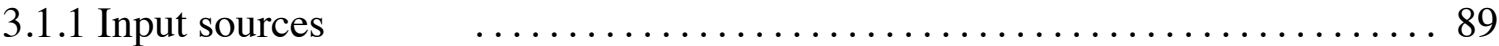

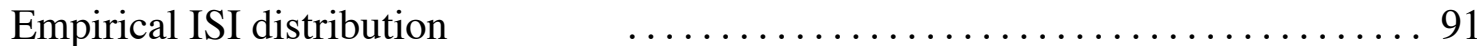

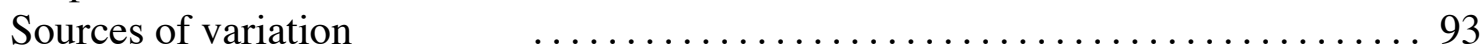

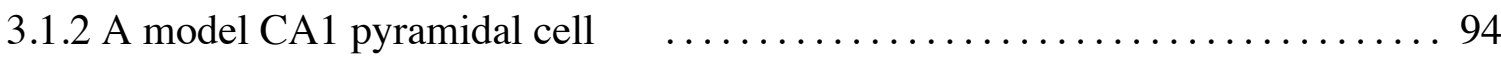

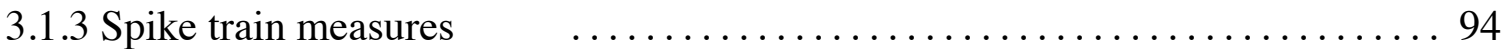

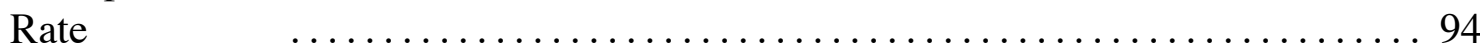

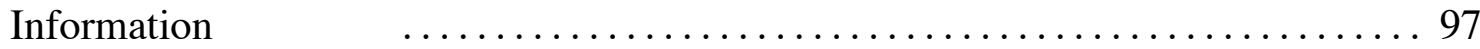

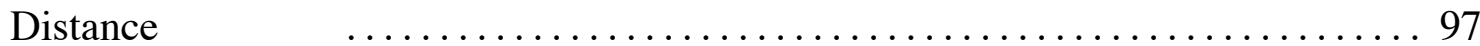

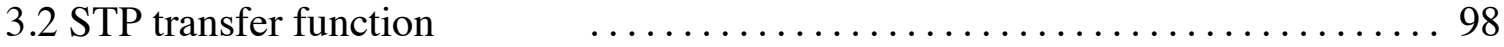

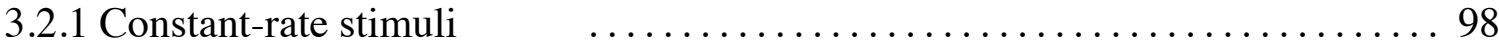

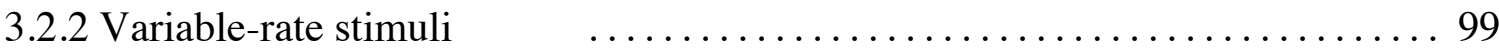

3.3 Effect of STP on Schaffer collateral processing $\quad \ldots \ldots \ldots \ldots \ldots \ldots \ldots$

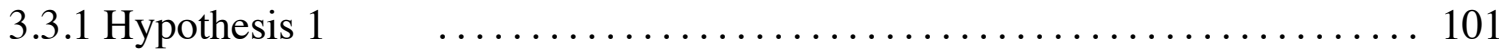

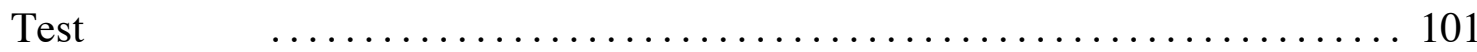

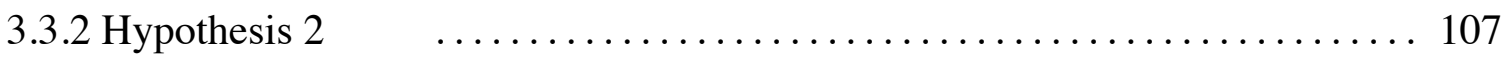

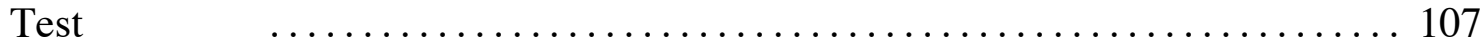

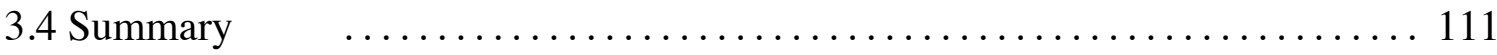

Chapter 4. Interpretation of STP at the Schaffer collateral 112

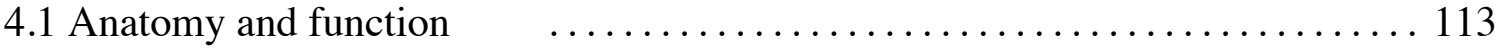

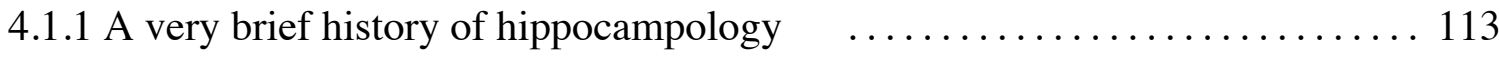

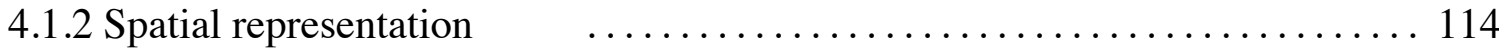

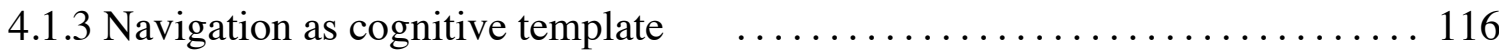

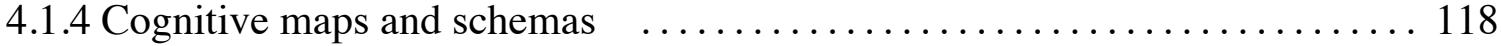

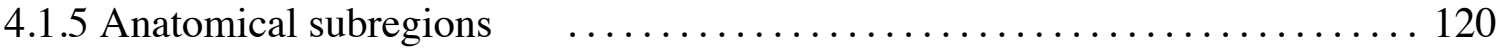

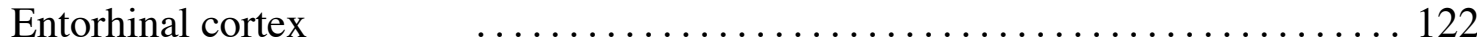

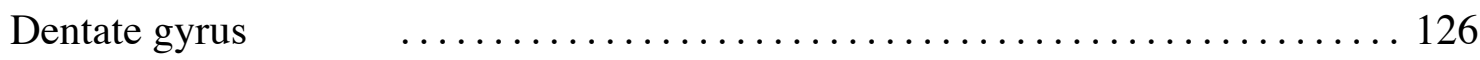

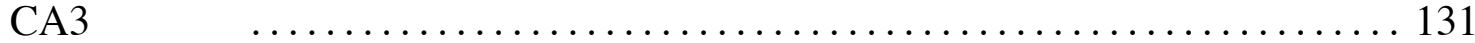

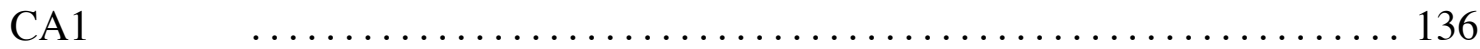

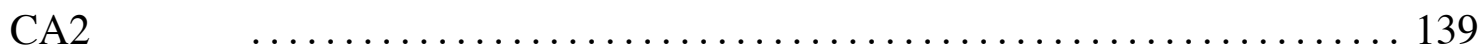

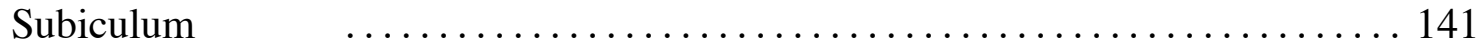

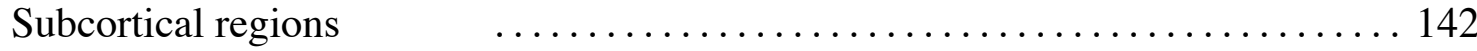

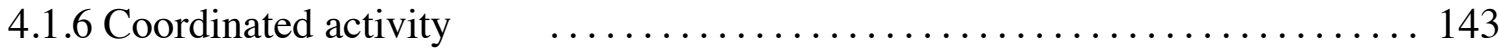

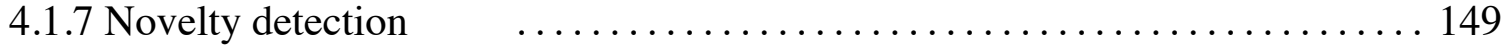




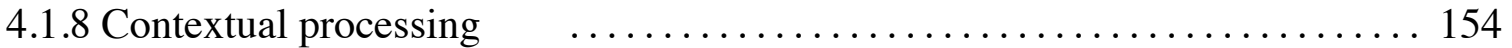

The context discerning multifunction network $\quad \ldots \ldots \ldots \ldots \ldots \ldots \ldots \ldots \ldots$

4.2 What does this have to do with STP at the Schaffer collateral? $\quad \ldots . .159$

4.3 Conclusion $\quad$.............................................. 169

$\begin{array}{ll}\text { References } & 172\end{array}$

Appendix A: DSCI model equations $\quad 204$

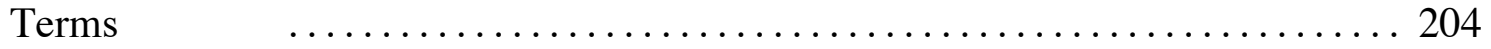

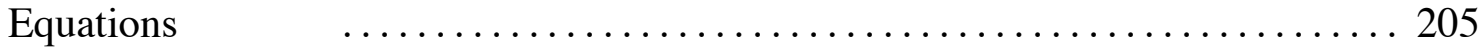

Appendix B: The Hill equation $\quad 209$

Appendix C: Optimization procedure $\quad 213$

Appendix D: CA1 pyramidal cell model $\quad 217$

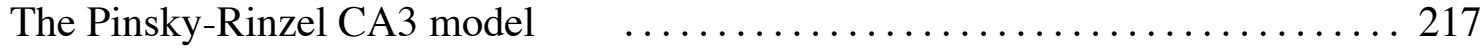

Parameter values published in [Pinsky and Rinzel, 1994] $\quad \ldots \ldots \ldots \ldots . \ldots 220$

Parameter values used to recover $\mathrm{CA} 3$ behavior $\quad \ldots \ldots \ldots \ldots \ldots \ldots \ldots 222$

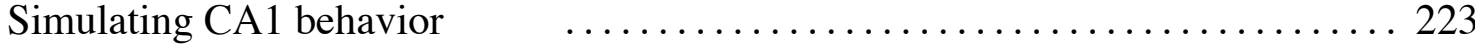

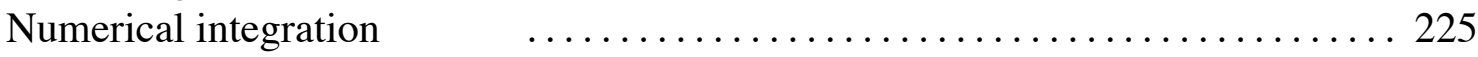

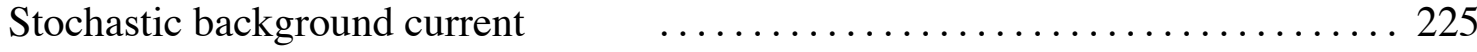

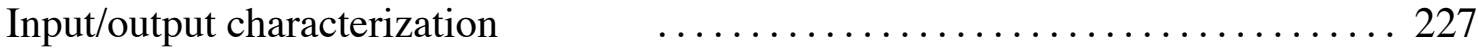




\section{List of Tables}

2.1 Parameters of the 2F3D model trained on ordered data 32

2.2 Parameters of the 2F3D model trained on complex data 33

2.3 The DSCI model parameter ranges 68

2.4 Optimized parameters for the DSCI model 69 


\section{List of Figures}

1.1 Illustration of presynaptic vesicle fusiona and recycling 3

1.2 Equivalent circuit representation of a section of neural membrane 14

1.3 Cut-away view of the human Limbic system 18

1.4 The hippocampal formation sits atop the cortical hierarchy 20

1.5 Block and schematic diagrams of hippocampal and neocortical connections 20

1.6 Schematics from Fig. 1.5 mapped onto rat brain anatomy 21

2.1 Normalized synaptic gain from ordered 24 train stimulation of the Schaffer collateral

2.2 Normalized synaptic gain from complex train stimulation of the Schaffer collateral

2.3 A descriptive 2 F3D model trained on ordered data 31

2.4 The model shown in Fig. 2.3 applied to complex data 32

2.5 A descriptive 2 F3D model trained on complex data 33

2.6 The model shown in Fig. 2.5 applied to ordered data 34

$\begin{array}{lll}2.7 & \text { DSCI model correlations to all data } & 70\end{array}$

2.8 DSCI model fit to training data from data set \#1 71

2.9 DSCI model fit to testing data from data set \#1 71

2.10DSCI model fit to training data from data set \#2 72

2.11DSCI model fit to testing data from data set \#2 72

2.12Residual calcium at spike times in data set \#1 73

2.13Calcium dependency of vesicle release probability in the DSCI model 75

2.14Vesicle pool populations at spike times in data set \#1 76

2.15Synaptotagmin isoform complements at spike times in data set \#1 77

$2.16 P_{\text {rel }}$ vs. (Syt7:Syt1) ratio at spike times in data set \#1 78 
$2.17 P_{\text {ves }}$ vs. (Syt7:Syt1) ratio at spike times in data set \#1 79

2.18Parameter sensitivities $\quad 81$

2.19Parameter sensitivities $\quad 82$

2.20Parameter sensitivities $\quad 83$

2.21 Parameter sensitivities $\quad 84$

2.22Parameter sensitivities $\quad 85$

3.1 Histogram of the spike times for a sample 90 input having 75 spike trains in three segments

3.2 Rastor plot of the spike times for a sample 91 input having 75 spike trains in three segments

3.3 Contour plot of the [Frerking et al, 2005] empirical ISI distribution 92

3.4 CA1 model output spike rate vs. time 95 response (STP - nonSTP) for DSCI model \#1

3.5 CA1 model output spike rate vs. time 96 response (STP - nonSTP) for DSCI model \#2

3.6 Input rates for responses shown in Figs. 3.4 and 3.5 96

3.7 Gain response of DSCI STP to constant-ISI inputs 99

3.8 ISI-dependent DSCI STP gain for variable-ISI stimuli at $1 \mathrm{~Hz}$ mean rate 100

3.9 Responsive CA3 ISI distribution at $1 \mathrm{~Hz}$ mean rate 102

3.10Approximate non-responsive CA3 ISI distribution at $1 \mathrm{~Hz}$ mean rate 102

3.11Difference of distributions shown in Figs. 3.9 and 3.10

3.12Gain and phase of filter corresponding 105 to the group delay fit to DSCI STP gain

3.13Gain and phase of filter corresponding to the gain fit to DSCI STP gain 106

3.14DSCI STP gain for different values of ISI $(t-2)$

3.15Slopes and offsets of linear fits of the test statistic defined in Eqn.(3.6) 110

3.16Same as in Fig .3.15, except that background noise was modulated at $25 \mathrm{~Hz} \quad 110$

$\begin{array}{ll}4.1 \text { Basic cortic-hippocampal connections } & 121\end{array}$ 
4.2 Jones and McHugh' s proposed hippocampal encoding model 146

4.3 Jones and McHugh' s proposed hippocampal consolidation model 146

4.4 Jones and McHugh's proposed hippocampal recall model 147

4.5 Hasselmo and Eichenbaum's proposed 158 functional organization of the hippocampus and EC

4.6 Architecture of the context discerning multifunction network 158

4.7 Diagram of a proposal for interpreting hippocampal processing 165 


\section{Preface}

Computation in the nervous system relies upon efficient communication between neurons throughout the brain and body. The large distances and tight packing between neurons requires a communication system that can rapidly transmit signals with minimal loss and effective isolation. Evolution has met this challenge by discretizing neuronal signals into electrical impulses that self-propagate along insulated lines. The nervous system has co-evolved a sophisticated encoding and decoding system that manages to work around the loss of resolution imposed by the signal discretization. A longstanding goal in neuroscience has been to decipher the spiking code, thereby allowing a record of neural computation to be reconstructed from observable spike patterns. If achieved, this would enhance our understanding of how the brain performs computations that enable the remarkable integration of sensory and motor functions, as well as the many processes that we take for granted, but that still elude our understanding [Abbott, 2008].

The physiological processes that are engaged in spike encoding are largely understood, and are characterized by systems of differential equations. Spike decoding, on the other hand, has yet to be characterized to the same level of biophysical detail, primarily because of the difficulty in observing the cellular processes involved. However, experimental advances have begun to clarify the mechanisms responsible for spike decoding, making it possible to begin to accurately model the process. 
Although details of spike decoding are only now starting to be understood, the process has long been known to involve neurotransmitter diffusion across the synaptic junctions between neurons. Both the release and uptake of neurotransmitters are tightly regulated processes that systematically alter the synaptic gain. It appears that these systematic variations in synaptic gain are the phenomena that enable the decoding of information in spike trains [Abbott and Regehr, 2004; Whitlock et al, 2006; Durstewitz, 2009; Rotman et al, 2011]. The release and uptake of neurotransmitters can be modeled as separate, but interacting, presynaptic and postsynaptic systems.

In the postsynaptic cell, neurotransmitter reception regulates synaptic gain on long time scales through changes referred to as "long-term plasticity" (LTP) [Lisman, 2009]. The dynamics of neurotransmitter reception are characterized by various kinetic models, and can be approximated by simple functions [Destexhe et al, 1995]. Progress has been made toward describing the modulation of neurotransmitter reception through the coincidence of electrical and chemical activity in the presynaptic and postsynaptic cells [Pawlak et al, 2010].

Variations in the probability of presynaptic neurotransmitter release, referred to as "short term plasticity" (STP), are a form of dynamic gain that does not permanently change synaptic efficacy [Zucker and Regehr, 2002]. In recent years, new details of the presynaptic processes responsible for neurotransmitter release have been reported [Sudhof and Rizo, 2011], but these details have not yet been successfully incorporated into a model of presynaptic spike decoding. This dissertation presents a new model of 
STP that synthesizes the current understanding of neurotransmitter release as it applies to spike-synchronous synaptic gain. The model we refer to as the "dual sensor competitive interaction" (DSCI) model.

The DSCI model of short term plasticity is used to address questions about presynaptic information processing, including whether short term plasticity filters spike patterns that convey behaviorally relevant signal expression in the brain (specifically in the hippocampus). In addition, this dissertation examines the effect of STP on the inputoutput function of a model excitatory neuron. Finally, this dissertation speculates upon the system-level role of STP in the hippocampus, using contextual organization as a paradigm for episodic memory formation.

It is anticipated that the DSCI model will advance the project of deciphering the neural communication code, enabling advances in neural prosthetics and elucidating how pathologies such as alzheimers, epilepsy, schizophrenia, and fragile-X syndrome disrupt neural processing. Furthermore, the DSCI model could be unified with postsynaptic models of neurotransmitter dynamics to examine the interactions between STP and LTP, thereby fully addressing questions about spike decoding in memory formation and learning. 
C H A P T E R 1

\section{Neural Systems}

The brain is a powerful, adaptable, and redundant computational engine [Hopfield, 1999; Haykin, 1999]. These characteristics of the brain are achieved through the distribution of computations across vast networks of neurons [Kanerva, 1993; Chklovskii et al, 2004; Nelson and Turrigiano, 2008]. The distribution of processing in the neural networks of the brain is recognizable on at least three scales. At the highest level, distributed processing in the brain involves the coordinated interaction between regional systems that perform operations such as multimodal sensory binding, action planning, recollection, and recognition. Consciouness emerges from the collective behavior of the regional systems. Within a region, networks of microcircuits with specialized neurons perform lower-level operations, such as constructing unimodal sensory representations from elemental configurations. Finally, computation is distributed across the cellular processes of individual neurons in the networks of the microcircuits. Section 1.1 introduces the general intra-neuronal level of processing, followed in section 1.2 by the regional and inter-neuronal levels of processing specific to the systems discussed in this dissertation. 


\subsection{Intra-neuronal systems}

Computation at the scale of a single neuron is distributed across three main cellular structures: the synapse, the dendrite, and the soma/axon. Synapses transform presynaptic spikes into postsynaptic potentials (PSPs), the dendrite integrates and filters PSPs from its array of synapses, and the cell body (soma) generates spikes as a function of the dendritic signal. Spikes are then transmitted by the axon to other cells. These three subsystems are introduced to provide a context for the model of short term synaptic plasticity (STP) presented in chapter two.

\subsubsection{The synapse}

Synapses are specialized zones between nerve cells wherein signals can be reliably transmitted through the extra-cellular cytoplasm. Synaptic signals are transmitted across a narrow cleft between the presynaptic and postsynaptic terminals via chemical neurotransmitters that are exocytosed from tiny vesicles in discrete amounts, or quanta. The amplitude of the postsynaptic response, $R$, is given by the relation, $R=n p q$, where $n$ is the number of quantal release sites, $p$ is the vesicle release probability, and $q$ is the amplitude of the postsynaptic potential produced by a quanta of neurotransmitter [Zador, 2001; Stevens, 2003].

The probability of neurotransmitter release is regulated by a "release mechanism" that consists of a suite of specialized proteins that manipulate the electrostatic energy barrier associated with lipidic membrane vesicle exocytosis [Rizo and Rosunmund, 2008; Sudhof and Rizo, 2011]. Fig. 1.1 illustrates the main features of the synaptic 
vescicle cycle, whereby neurotransmitter is packed, stored, and released.

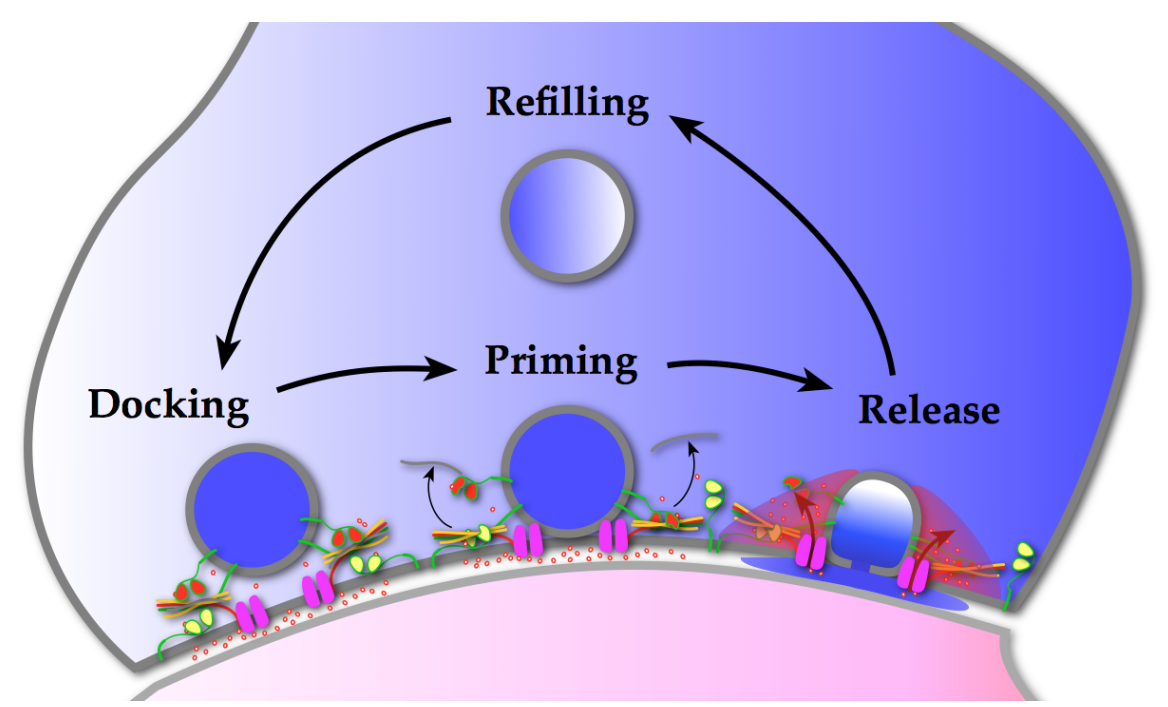

Figure 1.1. Illustration of presynaptic vesicle fusion and recycling.

The synapse is somewhat paradoxical in that it is a tightly regulated, yet probabilistic system [Goda and Sudhof, 1997; Brunger, 2006; Branco and Staras, 2009]. The probabilistic nature of neurotransmitter release might be partly explained by the inherent difficulty of controlling the process of lipid membrane fusion [Jahn, et al, 2003]. However, a certain amount of noise can be advantageous to some computations [Mayor and Gerstner, 2004; Averbeck et al, 2006; Rolls and Deco, 2010], so it is also possible that probabilistic release could be an evolutionarily conserved feature of neurotransmission. At the core of the dual sensor competetive interaction (DSCI) model, lies the proposition that synapses take advantage of the inherent stochasticity in the subcellular environment to fine tune neural information processing. 


\section{Vesicle fusion and release}

Presynaptic vesicle fusion is synchronized to the incoming action potentials by a group of proteins called the SNARE (soluble N-ethylmaleimide-sensitive fusion protein attachment receptor) complex, that hold neurotransmitter-containing vesicles in close proximity to the presynaptic plasma membrane at the active zone [Brunger, 2006]. At low calcium concentrations (as exists in the cell prior to an action potential), the SNARE complex is primed, but membrane fusion is prevented by a high-affinity SNARE-binding clamp (either complexin [Brunger et al, 2009] and/or apo-synaptotagmin [Chicka and Chapman, 2009]). At high calcium concentrations (as elicited by an action potential), the clamp is relieved by a low-affinity SNARE-binding trigger protein (Ca-synaptotagmin) that catalyzes membrane fusion within $500 \mu \mathrm{sec}$ of calcium entry, thereby synchronizing neurotransmitter release with action potentials [Yang et al, 2010]. The DSCI model characterizes STP as the competitive interaction between two isoforms of synaptotagmin that compete for access to binding sites on the SNARE complex.

\section{Chemical neurotransmission}

Neurotransmitters are mostly amino acids or monoamines, but there are also neuroactive peptides that can be released in conjunction with the small molecule neurotransmitters. Two neurotransmitters, glutamate and $\gamma$-aminobutyric acid (GABA), respectively account for most of the excitation and inhibition in the brain. Other neurotransmitters are used for various purposes, such as acetylcholine for activating muscles, and 
dopamine for signaling reward. Neurons can be characterized by the neurotransmitters that they release (Dale's principle), and accordingly there are various systems in the brain that generate specific neurotransmitters for signaling or modulation. The specialized neurotransmitters, including dopamine, serotonin, norepinephrin, acetylcholine, and several others, are produced in specific brain regions that innervate many other parts of the brain. Some specialized neurotransmitters modulate activity at synapses by remaining in the synaptic cleft for an extended period of time instead of getting absorbed or broken down.

Chemical synapses appear to have been adapted from more primitive structures [Sakarya et al, 2007]. The postsynaptic process has a number of conserved proteins that originally functioned as chemosensors in sea sponges [Miller, 2009]. Presynaptic systems that involve vesicle exocytosis likely co-evolved with chemosensors [Frerking, personal communication]. Both processes are ancient and fundamental to information processing important for organism survival. As evidenced by a general increase in synaptic complexity that trends with phylogenetic complexity [Emes et al, 2008], the chemical synapse likely provided life with the material for functional nervous system elaboration that an electrical synapses, though faster and more efficient, might not have. One of the functional elaborations of chemical synapses is the fine tuning of synaptic efficacy on arbitrary time scales. 


\subsubsection{Postsynaptic potentiation}

The postsynaptic response to neurotransmitters is determined by specific receptors, which in turn initiate events that culminate in postsynaptic activity, including the generation of postsynaptic potentials (PSPs). PSPs are passive voltage pulses with smaller amplitude and longer temporal extension than spikes, and can be either excitatory (EPSP) or inhibitory (IPSP), depending on which ion channels are engaged in response to the specific neurotransmitter. EPSPs raise, or depolarize the post-synaptic membrane from the non-zero resting potential, while IPSPs decrease, or hyperpolarize the membrane potential.

The passive propagation of the postsynaptic potential is described by the cable equation: $\lambda^{2} \partial_{x, x} V=\tau \partial_{t} V+V$, where $\lambda$ is the length constant of the dendrite, and $\tau$ is the membrane time constant. The electrical properties and geometry of the dendritic arbor determine the passive spatial and temporal filtering of the dendrite. Dendritic arbors can be approximated with finite "compartments" having simple geometrical shapes.

The number, size, location and kinetics of the synaptic inputs on the dendritic arbor influence the state of the PSP, and therefore contribute to the computational function of the neuron. For example, distal dendritic inputs are typically more attenuated than proximal inputs, unless augmented by active propagation [Magee, 2000]. However, if coincident with stronger signals, weaker inputs can be associatively enhanced. The size of the synapse also determines the impedance of the dendritic input. For instance, smaller synapses have higher impedance, and couple less effectively to the dendrite. 
Finally, receptor kinetics vary by type. For instance, $N$-Methyl-D-aspartate (NMDA) receptors generate a weaker peak signal than Alpha-amino-3-hydroy-5-methyl-4 isoxazole proprionic acid (AMPA) receptors, but the NMDA response is about five times longer than the AMPA response [Liaw et al, 2000]. AMPA receptors are the primary receptor of excitatory signals in the brain, and NMDA receptors are thought to mediate long term plasticity (LTP) by regulating AMPA receptor expression [Kandel, Schwartz, and Jessel, 1991].

\subsubsection{Synaptic plasticity}

Changes in synaptic efficacy are categorized into long-term and short-term processes. Long-term plasticity is thought to be responsible for persistant learning and memory [Siegelbaum and Kandel, 1991], while short-term plasticity most likely effects the characteristics of signal transmission between neurons [Abbott and Regehr, 2004].

\section{Long-term plasticity}

The sensitivity of the postsynaptic terminal to neurotransmitter is determined by the type, number and conductance of receptors, which can vary by activity-dependent processes that are generally referred to as long term plasticity (LTP). LTP has two temporal phases: early (E-LTP) and late (L-LTP), that are separated in time by about an hour after the triggering activity [Nguyen and Kandel, 1997]. Early LTP can be induced by strong membrane depolarization resulting from high frequency spike trains or coordinated presynaptic and postsynaptic activity [ibid]. The most likely receptor- 
based explanation for E-LTP involves an increase in the conductance of AMPA receptors, initiated by a rise in calcium concentration in the postsynaptic terminal resulting from an increase in the calcium conductance of NMDA receptors due to coincidental presynaptic and postsynaptic spiking [Graupner, 2010]. Higher AMPA receptor conductance subsequently increases the postsynaptic response to neurotransmitter release. Late-phase LTP partly results from an increase in the number of AMPA receptors, which requires the induction of protein synthesis pathways by transcription factors [ibid]. Neuromodulators can enhance the effect of late LTP; dopamine in particular may be responsible for the coupling of early to late LTP [Pawlak et al, 2010].

Coincidental presynaptic and postsynaptic spiking activity is sufficient to trigger early LTP, suggesting that the process instantiates the Hebbian theory of synaptic modulation [Lisman et al, 2011], referred to as spike-timing-dependent plasticity (STDP), that implicates coincidental presynaptic and postsynaptic firing [Markram et al, 1997]. The postsynaptic spike back-propagates up the dendrite from the soma and affects molecularly tagged synapses. The relative timing of presynaptic and postsynaptic spikes determines the direction of the plasticity: presynaptic spiking prior to postsynaptic spiking (within about 20ms) results in LTP, while postsynaptic spiking prior to presynaptic spiking results in LTD [Roberts and Leen, 2010].

\section{Short-term plasticity}

Short term plasticity (STP) has not received as much attention as LTP, especially in artificial neural networks, despite the fact that STP can alter the gain of the signal as 
much as LTP, albeit on shorter time scales [Koch, 1999]. Perhaps the reason for the lack of attention in the artifical neural network community is that not much is known about what STP does. However, studies that have included STP in neural network models have shown an increase in the functionality of the neural network models [Amit and Mongillo, 2003; Buonomano, 2000; Fortune and Rose, 2001; Klyachko and Stevens, 2006; Maass and Markram, 2002; Mejias and Torres, 2007; Natschlager et al, 2001; Silberberg et al, 2004; Torres et al, 2007].

Proposals for the function of STP include a mechanism to provide spike frequency adaptation [Koch, 1999], sensitivity to changing patterns, and rapid switching among stored patterns [Pantic et al, 2002; Cortes et al, 2004; Marro et al, 2007; Torres et al, 2005; Cortes et al, 2006]. Also, STP has been proposed to improve associativity [Marro et al, 1998], as a means of gain control [Varela et al, 1997], coincidence detection [Senn et al, 1998], and decorrelation [Goldman et al, 2002]. STP has also been proposed to allow a single axon to encode information as a spatio-temporal pattern across its terminals, rather than a single sequence of spikes across all terminals [Liaw and Berger, 1999]. More recently, a model for transforming temporal into spatial patterns in a decision making process using STP has been proposed [Deco et al, 2010]. Another recent and intriguing proposal, is that STP optimally estimates the presynaptic membrane potential from the spike train history [Pfister et al, 2010].

All of the proposed functions of STP share in common an activity-dependent alteration of synaptic gain. Generally, activity-dependent gain adjustments can be under- 
stood in terms of nonlinear filtering [Maass and Sontag, 2000]. The fact that STP can instantiate an arbitrary nonlinear filter may imply that synaptic dynamics can extract relevant details from a noise-corrupted spatio-temporal signal. It may also be the case that specific signal properties are used for transmitting information in different operating regimes of the brain and that filtering is a necessary component in the detection of these signals. There may also be a specific alteration of features, such as sharpening or edge detection that are facilitated by synaptic dynamics.

Additionally, the characteristics of STP are tuned to specific brain regions [Dittman et al, 2000]. For example, climbing fiber synaptic dynamics are characterized by pure depression, while parallel fiber synapses are characterized by pure facilitation (climbing fibers and parallel fibers are located in the cerebellum). Schaffer collateral synapses are characterized by a mixture of facilitation and depression. The first inference to draw from the regional specificity of short term dynamics is that STP is almost certainly not an epiphenomenon of synaptic transmission. Regional specificity could also imply that there are particular computational requirements at synapses in different regions in the brain. For neural modeling, the presence of specific forms of STP throughout the brain means that information processing in the brain will not be completely understood until STP is included in the models.

\subsubsection{Spike initiation}

The soma is the locus of decision-making in the neuron. The instantaneous amplitude of the PSP at the soma affects the dynamical state of the neuron, driving it through 
transitions in behavioral regimes. Spiking is a transition, or bifurcation, that occurs due to the interaction of nonlinear ion conductances in the membrane of the neuron [Izhekevich, 2006]. A bifurcation is a qualitative change in behavior of a dynamical system. Active ion channels of several ionic species establish an electrochemical dynam ical system capable of bifurcating in numerous ways, for instance from a stable equilibrium state to a limit cycle attractor [ibid].

The essential condition required for spiking is the presence of at least one amplifying ionic current, and one resonant ionic current. The amplifying current creates a positive feedback loop; the resonant current creates a negative feedback loop [Izhekevich, 2006]. Spiking occurs if the positive feedback loop is faster than the negative feedback loop. In the Hodgkin-Huxley $(\mathrm{HH})$ model of action potential generation, an inward sodium conductance controls the amplifying current, and a slower outward potassium conductance controls the resonant current.

The time variance in the conductances is caused by voltage-dependent gating mechanisms in the ion channels. In the original $\mathrm{HH}$ model, the behavior of the gates is described with sigmoidal functions. For the sodium current, there are activation and inactivation gates, the behavior of which is described by opposing sigmoids that are respectively zero at both voltage extremes, and have different kinetics. There is a voltage range, or activation window, in which the product of the sigmoids is nonzero. As the voltage at the membrane increases into the range of the activation window, the sodium channels begin opening, further depolarizing the membrane, thus starting the 
positive feedback cycle. The positive feedback cycle terminates as the voltage approaches the other side of the activation window, effectively closing the sodium channels by engaging an inactivation mechanism.

The narrow shape of the spike is generated through an interaction with an outward potassium current that also turns on as the membrane depolarizes. The potassium current turns on at about the same time as the sodium current, but the potassium channel dynamics are slower, so the potassium current arrives at its maximum value later than the sodium current. The width of the spike is thus restricted to about 1 to $5 \mathrm{~ms}$. Following spike generation, there is a period of refractoriness during which the inactivation gates on the sodium channels slowly reopen over a period of about $10 \mathrm{~ms}$. Regeneration of the hyperpolarized state is performed against the concentration gradient of the ion species, so active pumps are employed to maintain the ion gradient over a long time scale.

\subsubsection{Neural models}

There are two general classes of models used to describe neuronal systems: spiking and non-spiking. Non-spiking models describe the output of a neuron as an analog signal intended to correspond to the rate of spikes emerging from a cell or group of cells. The activation function of most non-spiking neural network models is sigmoidal, simulating a typical neuronal input/output rate function. Non-spiking artificial neural network models are an efficient and powerful abstraction from the biological details of information transmission. The utility of non-spiking neural network models lies in the 
analytical and computational efficiency of the abstraction of the connectionism of neural networks. Artificial neural networks are mentioned, but not used in this study.

\section{Spiking models}

Spiking models are used when some degree of biological realism is required. The amount of realism depends on the aspects of the system being studied, and can range from rudimentary, as for example with the integrate and fire models, to quite precise, as with multi-compartmental models that closely mimic specific dendritic morphologies and channel densities. The spiking model used in this dissertation is a two-compartment conductance model (described in Appendix A).

Spiking models have a variety of forms. A large family of spiking models, including the integrate-and-fire, the leaky integrate-and-fire, and the Hodgkin-Huxley conductance model are variations on the law of capacitance, $q=C V$, expressed in differential form: $C \frac{d}{d t} V(t)=I-\sum_{i=1}^{N} I_{i}(t, V)$ [Dayan and Abbott, 2001]. The sum of the capacitive current, $C \frac{d}{d t} V(t)$, and ionic currents, $\sum_{i=1}^{N} I_{i}(t, V)$, (describing the flow of any number of ion species across the membrane), equals the total current, $I$, obeying Kirchoff's current law. Conductance models consider the neuronal membrane in terms of an equivalent electrical circuit, as illustrated in Fig. 1.2. 


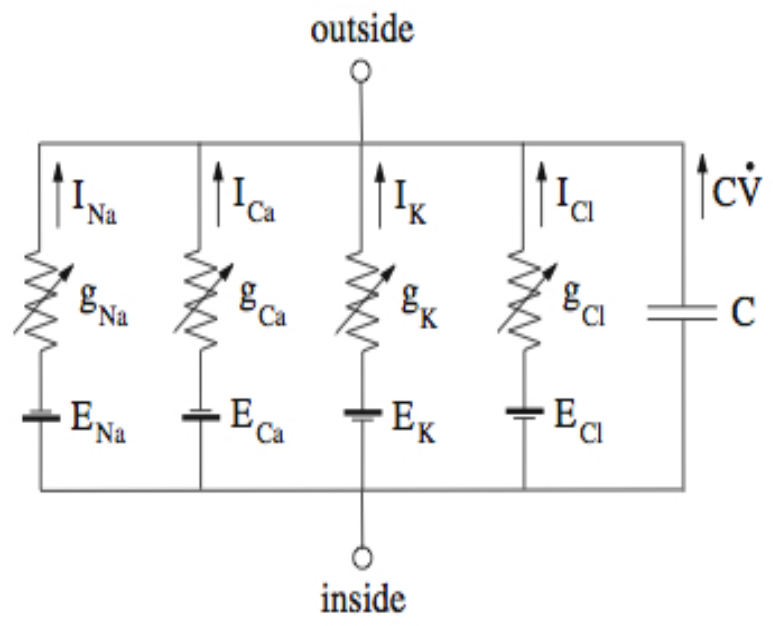

Figure 1.2. Equivalent circuit representation of a section of neural membrane as a sum of currents composed of variable resitors in tandem with batteries. Other than the channels, the membrane is non-conductive, so it has a capacitance. The variable conductances have voltage-dependent behavior that results in an impulsive current called an action potential. Adapted from: [Izhekevich, 2006]

The ionic currents, $I_{\text {ion }}$, on a patch of neuron membrane are described by the probabil ities that the channels are open or closed based on time and voltage-dependent activatio $\mathrm{n}$ and inactivation variables, $m$ and $h$ :

$$
I_{\text {ion }}=\bar{g} m^{a} h^{b}\left(V-E_{\text {ion }}\right), \quad m, h \in[0,1] .
$$

$E_{\mathrm{ion}}$ is the reversal potential of the ion channel current, the voltage at which the concentration and electrical potential gradients of the ionic species are in balance. The force driving the ionic current is given by $\left(V-E_{\text {ion }}\right)$. The ion channel has a maximal conductance value given by $\bar{g}$. The kinetics of the activation and inactivation variables are described by first order O.D.E.s. (showing only the activation function here),

$$
\frac{d m}{d t}=\frac{m_{\infty}(V)-m}{\tau_{m}(V)} .
$$


The voltage dependence of the activation and inactivation variables is expressed in the activation and inactivation functions, $m_{\infty}(V)$ and $h_{\infty}(V)$, and in the time constant functions, $\tau_{m}(v)$ and $\tau_{h}(V)$. The activation and inactivation functions are usually sigmoidal:

$$
m_{\infty}(V)=\frac{1}{1+\exp \left\{\left(V_{1 / 2}-V\right) / k\right\}},
$$

and the voltage-dependent time constants are usually unimodal:

$$
\tau_{m}(V)=c_{\text {base }}+c_{\text {amp }} \exp \left\{\frac{-\left(V_{\max }-V\right)^{2}}{\sigma^{2}}\right\} .
$$

The conductance model described above for the ionic currents is in general a kinetic Markov model that can be applied to most any ionic channel, including neurotransmitter receptors [Destexhe et al, 2001]. The kinetics of AMPA, NMDA and GABA receptors are best described by multi-state kinetics that account for the processes of activation, inactivation, desensitization, saturation and priming. AMPA and $\mathrm{GABA}_{B}$ receptor kinetics can be simplified to a two-state model having a single term defining the open state probability, along with a voltage-dependent gating mechanism in the case of NMDA receptors, which have a magnesium block. $\mathrm{GABA}_{A}$ receptor kinetics require a four-state model due to a ligand-gated second messenger system. Presynaptic neurotransmitter release can also be described with kinetic models, but have not yet included mechanisms that successfully account for short term plasticity.

\subsection{Inter-neuronal systems}

Considered from the systems perspective, the brain is a network of networks, having regional and subregional specificity of nodes and connections [Macaluso and Driver, 
2005; Philips and Singer, 1997]. Generally, signals from sensory or regulatory systems are abstracted, through convergence and/or divergence, with transformation and/or association, as they are relayed through network nodes throughout specific processing regions [Haykin, 1999]. The system maintains a representation of the input signals consistent with feedback received from effector systems that change the state of the inputs in a controlled manner. The state representation is a function both of the inputs, and of the history of the representation [Meyer, 2012]. The representation may be influenced by recent and remote history, due to memory systems that retain information across several time scales.

One of the primary memory systems is the hippocampal formation. The hippocampus in particular operates by abstracting features from signals that originate in cortical association regions and subcortical limbic and brainstem regions, thereby allowing cortical/subcortical associational processing that facilitates efficient memory storage and recall [Rolls, 2010]. Since hippocampal anatomy is pertinent to understanding the Schaffer collateral in a broader context, the anatomy of the hippocampus is introduced in this section, and then is examined in more detail, along with some aspects of the physiology, in chapter three.

\subsubsection{The Hippocampus}

The DSCI model of STP was developed using data recorded from the hippocampus. The hippocampus is ideal for studying synaptic plasticity because the tissue is convenient to surgically isolate, the neurons are readily excitable, and the synapses are very 
plastic. The laminar organization of the collateral axons in the hippocampus makes it possible to stimulate large numbers of axons simultaneously, resulting in a large field Excitatory Post Synaptic Potential signal, or fEPSP, that reflects the bulk synaptic efficacy. Also, chemical antagonists of the postsynaptic receptors implicated in hippocampal LTP are available, so that STP can be isolated from LTP pharmacologically. Beyond these practicalities, the hippocampus is interesting from a systems perspective because of the central position that the hippocampus has in spatial awareness and in memory encoding and recall. Unique and robust neural signatures of behavioral correlates have been found in the hippocampus, making it possible to directly observe behavioral effects of circuit modifications, and thus to test specific hypotheses of hippocampal function.

\section{Basic anatomy}

The hippocampal formation is a cortical supramodal association area in the medial temporal lobe that receives higher-order input directly from the limbic association cortex [Cscicsvari et al, 2000]. The hippocampus is cortical tissue, but is part of the limbic system, which borders the neocortex (Latin, limbus: border or rim), and is highly connected with the prefrontal cortex [Kandel, Schwartz, and Jessell, 1991]. The limbic system is known to be associated with aspects of emotion, including the management of endorphins, the "fight or flight" neurotransmitters, and dopamine, the "reward" neurotransmitter. The limbic system also modulates the activity of the autonomic nervous system. 


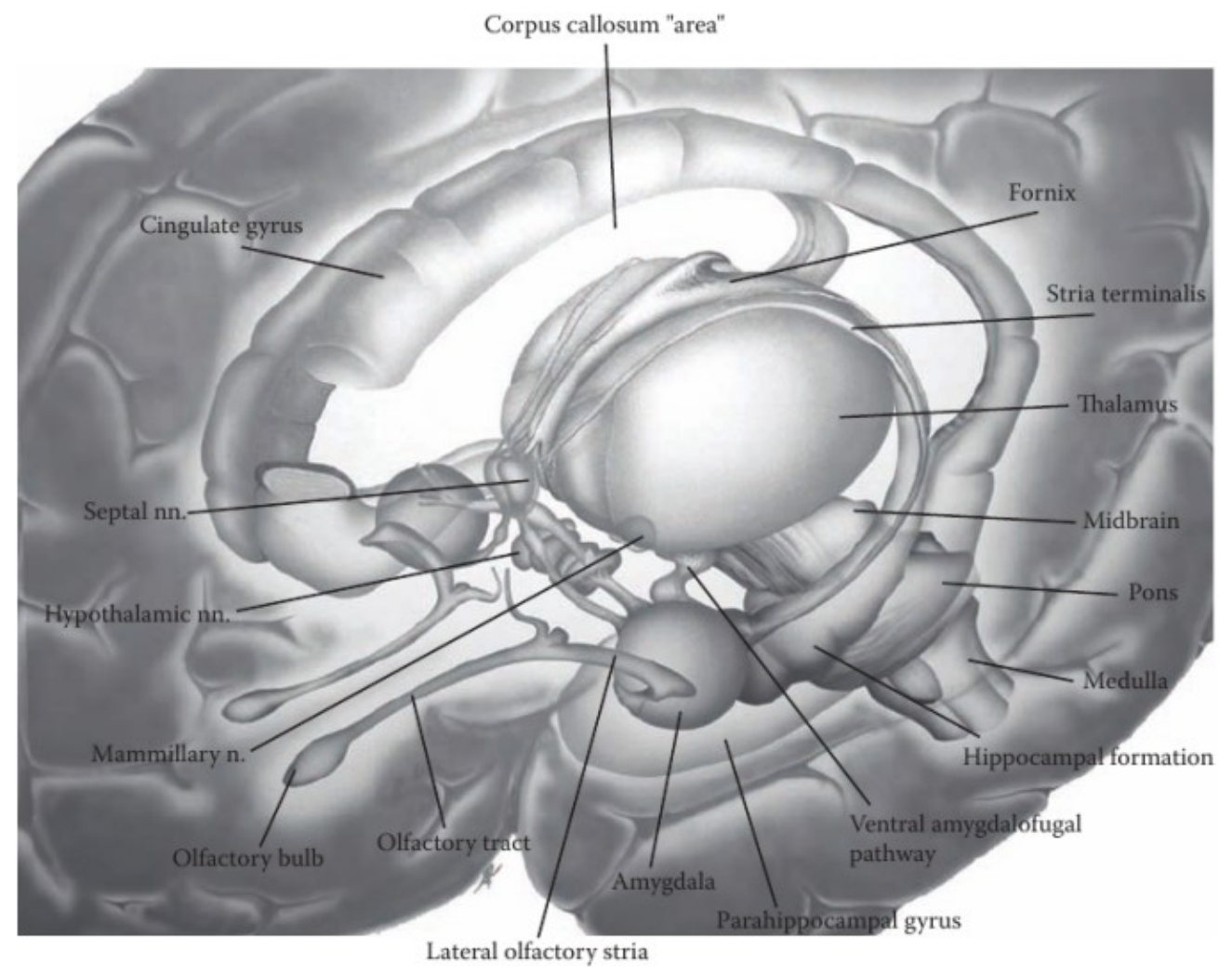

Figure 1.3. Cut-away illustration of the human Limbic system. Figure credit: [scribd.com]

The hippocampus receives highly processed, but mostly uncorrelated, input from all sensory modalities [Rolls and Treves, 1998], and is thought to interact with the neocortex in the process of consolidating and recalling long-term episodic memories [Shimamura, 2003; Anderson et al, 2007]. Figure 1.4 illustrates the bi-directional signaling pathways that originate in the sensory regions of the neocortex and converge on the hippocampus. The hippocampus proper is differentiated unambiguously into the dentate gyrus (DG) and cornu ammonis (CA) subregions that have been shown to play 
distinct roles in the encoding of consciously retrievable long-term declarative memories that are stored in the neocortex [Anderson et al, 2007; Stark, 2007].

Neocortical signals enter the hippocampus from the entorhinal cortex, layer two (ECII), and pass, via the perforant path (PP), to the dentate gyrus (DG), as well as directly to the Cornu Ammonis, area three (CA3). The DG projects to CA3, and from CA3 to CA1, completing a trisynaptic loop. The trisynaptic loop is complemented by two parallel circuits: a monosynaptic loop that originates in EC, layer three (ECIII) and terminates in CA1 via the temporoammonic pathway (TA), and a disynaptic loop that projects from both $\mathrm{EC} 2$ and $\mathrm{EC} 3$ onto $\mathrm{CA} 2$, and then from $\mathrm{CA} 2$, to $\mathrm{CA} 1$ [Chevaleyre and Siegalbaum, 2010]. The CA1 output projects to the subiculum, before terminating in the entorhinal cortex, layer five (ECV). The monosynaptic and trisynaptic loops are shown in schematic in Fig. 1.5 and mapped onto the rat hippocampal anatomy in Fig. 1.6. These parallel pathways operate in tandem to enable memory encoding and recall, but their exact interaction in these processes are still not known. 


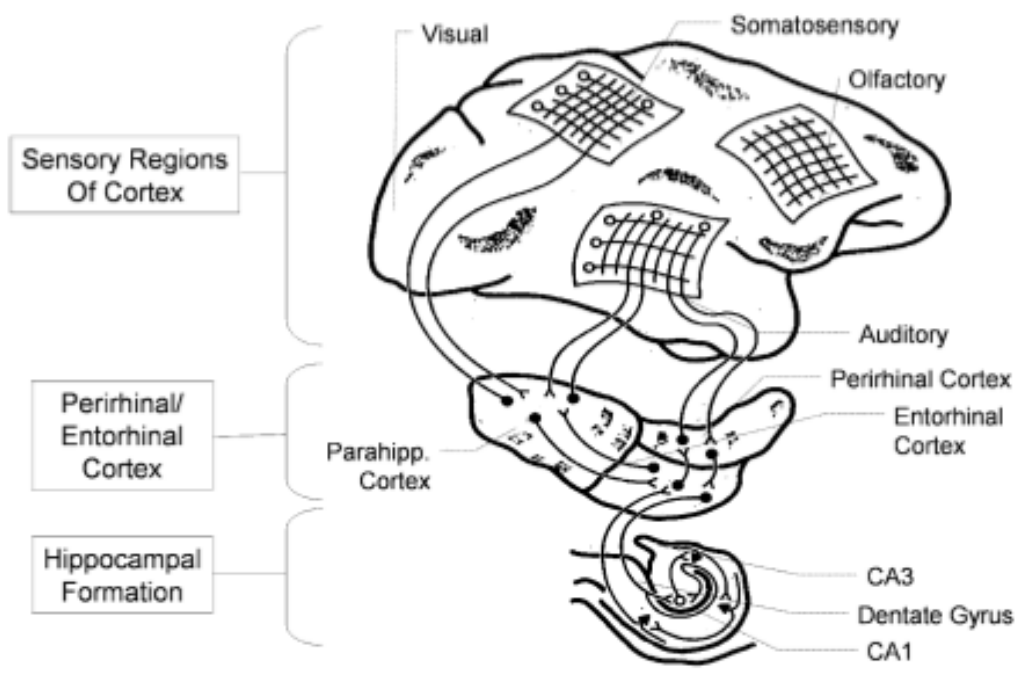

Figure 1.4. The hippocampal formation sits atop the cortical hierarchy and receives abstracted information from the sensory and associational cortices. Figure credit:

[Sweatt, 2004]
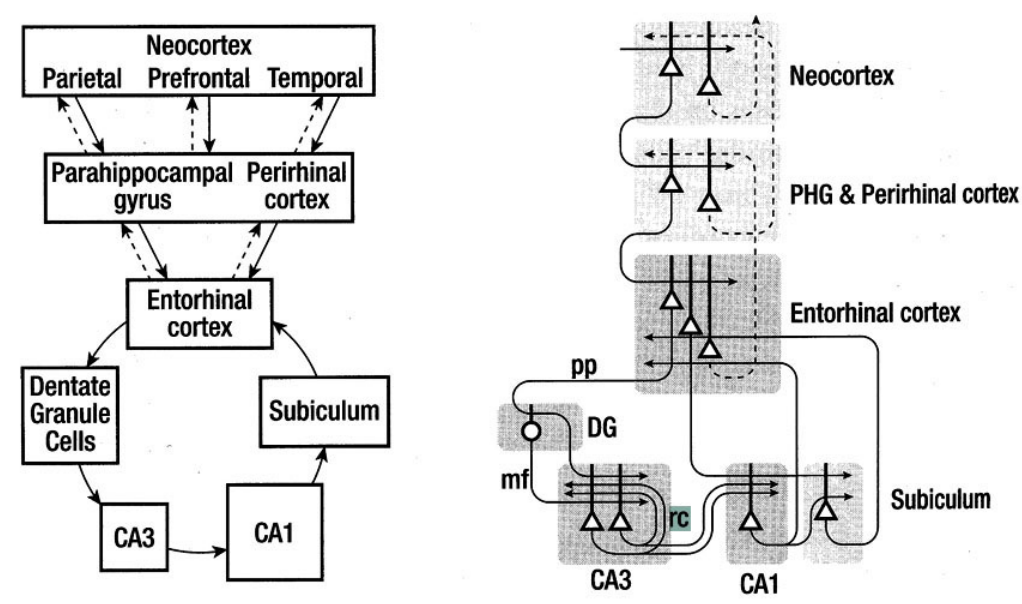

Figure 1.5. Block and schematic diagrams of hippocampal and neocortical connections. The tri-synaptic hippocampal processing loop is shown in the drawing on left (the $\mathrm{CA} 1 \rightarrow$ Subiculum connection is not counted among the synapses in the trisynaptic loop). Figure credit: [Rolls, 2007] 


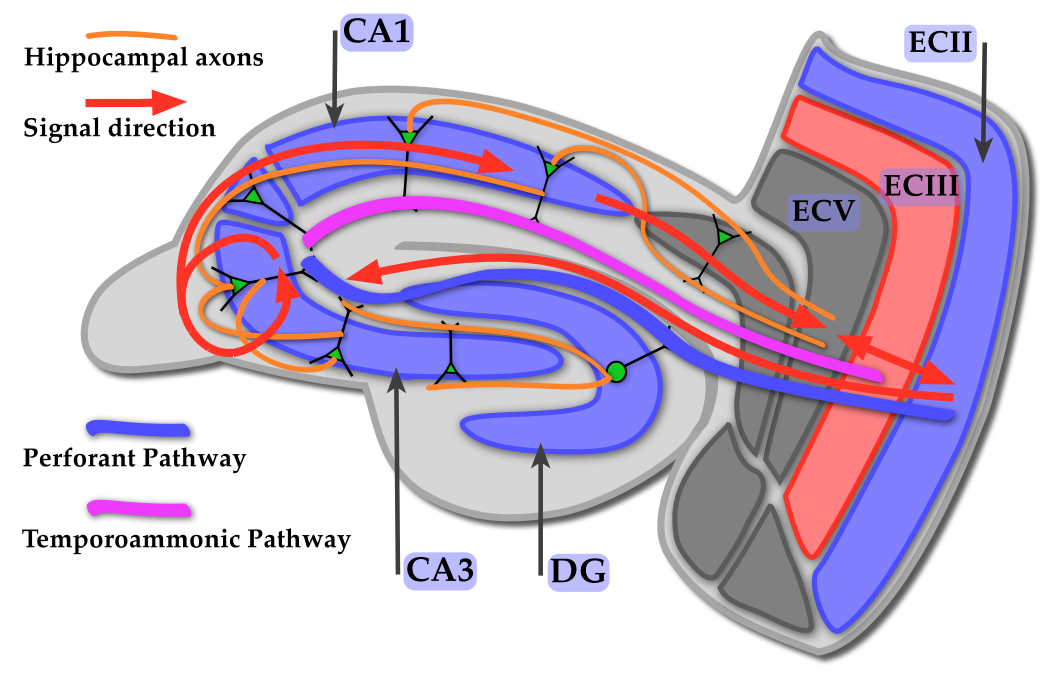

Figure 1.6. The schematics from Fig. 1.5 mapped onto the anatomy of the rat brain (minus the connections between EC and the rest of the neocortex). The monosynaptic $(E C I I I \rightarrow C A 1)$ and trisynaptic $(E C I I \rightarrow D G \rightarrow C A 3 \rightarrow C A 1)$ circuits shown. Only the principal cells are depicted.

\subsection{Summary}

The mechanisms and functions of STP in hippocampal processing at the Schaffer collateral are still a mystery. We would like to better understand the principles of synaptic dynamics, and how they contribute to the processing of signals that convey the code for episodic memories between the CA3 and the CA1.

Chapter two is a discussion of the efforts taken to describe synaptic dynamics, including the most successful published phenomenological and mechanistic models, followed by a description of the DSCI model of STP. 
C H A P T E R 2

\section{Modeling STP at the Schaffer collateral synapse}

\subsection{The Schaffer collateral}

The Schaffer collateral is a tract of collateralized axons that projects from region CA3 in the hippocampus and terminates on region CA1. The signals carried on the Schaffer collateral are crucial for a number of cognitive operations, and are finely tuned to provide appropriate stimulation of the CA1 [Ahmed and Mehta, 2009]. Several systems in the CA region, including an assortment of interneurons, several forms of plasticity, and at least two interacting timing mechanisms, exist to keep the CA region balanced between excitation and inhibition [Klausberger and Somogyi, 2008; Nelson and Turrigiano, 2008]. The deleterious effects of overstimulation in the hippocampus indicate the importance of keeping CA activity from becoming too excitatory [Onodera et al, 1986; Stief et al, 2007]. On the other hand, there must be a reason to keep the balance of activity close to such a sensitive regime, and from becoming overly inhibited [Atallah and Scanziani, 2009].

\subsection{Synaptic dynamics data}

For purpose of collecting data to fit and validate the STP model, measurements of synaptic dynamics at the Schaffer collateral synapses were made by recording the $f$ EPSP generated in response to axonal stimulation with $100 \mu$ s pulses delivered by a 
dipole electrode. The $f$ EPSP provides a signal of the synaptic activity from a large number of synapses, effectively averaging out much of the noise in comparison to single cell recordings, but at the expense of averaging out synapse-to-synapse variability. However, since the synapse-to-synapse variability would have to be averaged out anyway if whole-cell recordings were used, the $f \mathrm{EPSP}$ is a much more efficient measure ment [Frerking and Roberts, personal communication].

The slope of the $f$ EPSP signal effectively approximates the synaptic current through the law of capacitance, $C \frac{d V}{d t}=I$, providing a measure that is proportional to the presynaptic release probability, as inferred from the current. Since the $f$ EPSP signal becomes progressively contaminated by population spikes from the CA1 cells excited by the post synaptic potentials, the magnitude of the $f$ EPSP is determined by measuring the initial slope of the $f$ EPSP, where the signal is cleanest. The initial slope is also most characteristic of the synaptic current that is synchronous with the stimulation.

Traditionally, synaptic dynamics have been elicited with short trains of stimuli applied at constant intervals. However, constant-rate input trains do not sample the possible combinations of inter-stimulus intervals (ISIs) that are engaged by in vivo firing patterns. The complicated history-dependency of the response requires a broader range of ISIs to adequately sample the synaptic dynamics. Therefore, randomized input trains are more appropriate for sampling synaptic dynamics [Zador and Dobrunz, 1997]. Physiologically realistic ISIs can be drawn from distributions developed from recordings of spiking trains from active animals [Frerking et al, 2005]. Alternatively, 
actual spike trains can be used.

The input signals used for the development of the DSCI model consisted of eight constant-rate "ordered" ISI trains and two variable-rate "complex" ISI trains. The ordered trains each consisted of 40 stimuli at physiologically relevant frequencies, including, 0.2, 0.5, 1, 2, 5, 10, 20, and 50Hz (see Fig. 2.1). Each ordered train was preceded by three pulses at $0.1 \mathrm{~Hz}$ to establish a baseline level of synaptic gain. These trains were followed by a single stimulus after a 10 second interval, to capture the rebound activity, or post-tetanic potentiation (PTP). The complex ISI trains were recorded in vivo from rat CA3 pyramidal cells during a hippocampus-dependent task (Fig. 2.2). These trains consisted of about 60 stimuli with an average frequency of about $1 \mathrm{~Hz}$, preceded by 30 stimuli at a constant rate of $1 \mathrm{~Hz}$ to establish a physiologically relevant basal level of activity.
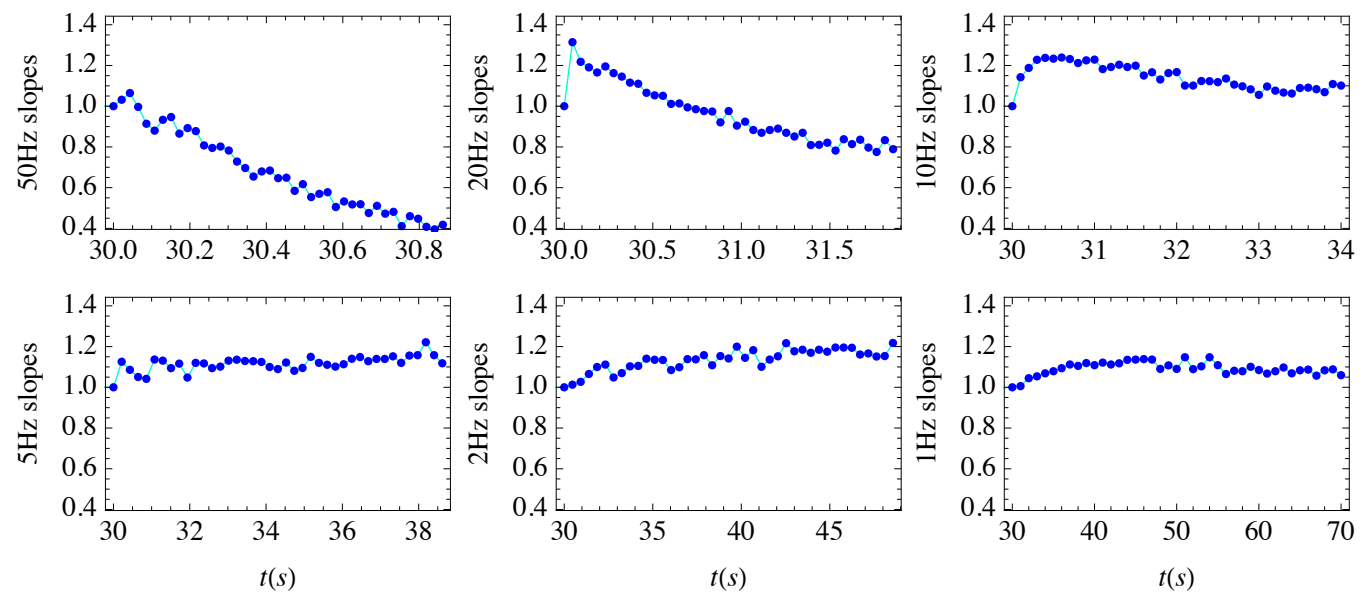

Figure 2.1. Normalized synaptic gain resulting from ordered train stimulation applied to the Schaffer collateral of a juvenile rat. The term "slopes" refers to the technique of measuring the initial slope of the fEPSP to derive the gain (see text). Note that the ranges on the gain axis are not equal in all of the panels. 

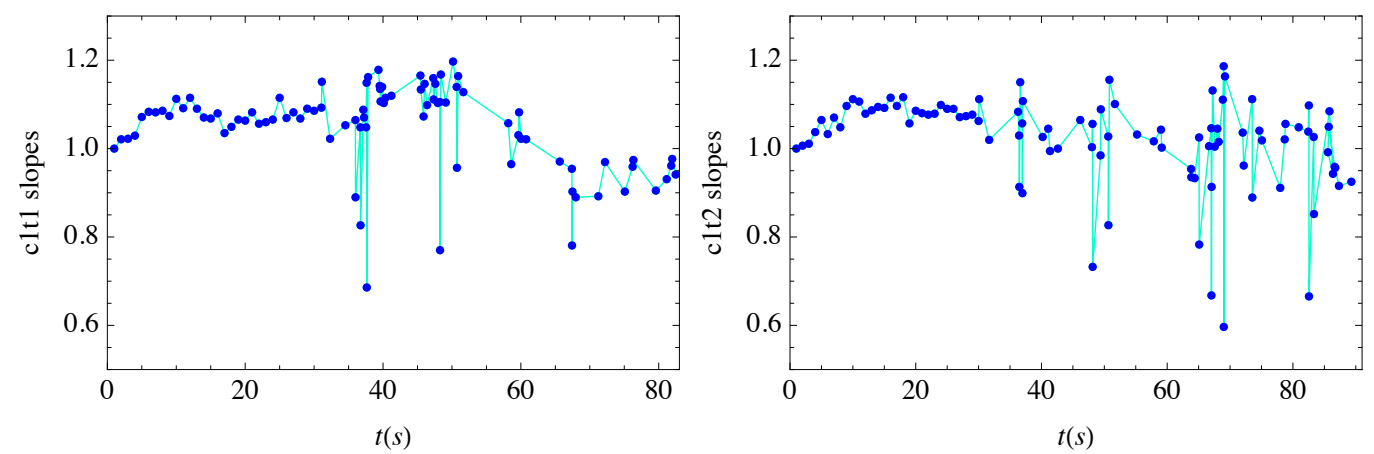

Figure 2.2. Normalized synaptic gain resulting from "complex" natural train stimulation applied to the Schaffer collateral of a juvenile rat.

All data was collected from juvenile rat hippocampal slices and prepared according to [Ohliger-Frerking et al, 2003]. In the slices, the Schaffer collateral axons were severed between areas CA3 and CA1 to remove the source of activity from the recurrent CA3 network. A bipolar stimulating electrode was placed directly in the Schaffer collateral on the CA1 side of the lesion to stimulate the axons. Two pharmacological agents were applied to the slices. The NMDA receptor antagonist, (2R)-amino-5phosphonovaleric acid (AP5), was applied to block NMDA receptors, effectively eliminating LTP and ensuring that only STP would be elicited during the stimulus train. Secondly, picrotoxin, a GABA antagonist, was used to block GABA receptors, thereby preventing potential contamination of the measured signal by IPSPs from interneurons. The recordings were made at $34^{\circ} \mathrm{C}$. Two complete sets of recordings, each including the constant rate and complex trains, were obtained under equivalent conditions, in unique experiments performed on different days. Each set of recordings was obtained in a single slice. Data collection was performed by Matt Frerking in 
2007. The data set will hereafter be referred to as the "Frerking data."

The synaptic gain was normalized to the first three points in the $0.1 \mathrm{~Hz}$ run-up to the constant-rate trains, and to the first point in the complex trains. The data shows the characteristic combination of facilitation and depression seen in Schaffer collateral STP (see Figs. 2.1 and 2.2). Repeated presentations of the same train yielded $r^{2}$ values between 0.8 to 0.85 . This value of correlation represents the upper limit of any expected fit, and higher values would indicate over-fitting by describing noise in addition to the data.

\subsection{Review of the STP modeling effort}

Most models of short term synaptic plasticity combine various independent facilitative and depressive processes to phenomenologically reproduce the range of dynamic behaviors observed in synapses throughout the brain [Varela et al, 1997]. The independent terms in these models are typically first-order O.D.E.s with instantaneous forcing terms. None of the published phenomenological models have yet shown a characterization of combined facilitory and depressive synaptic dynamics that generalizes to arbitrary ISIs. The main difficulty seems to be in capturing use-dependency across all conditions. Simpler phenomenological models don't track vesicle usage explicitly, but instead represent use-dependency through the interaction of the facilitative and depressive terms. In particular, these models have trouble simultaneously describing normal operating conditions, where vesicle reloading can keep up with vesicle depletion, and the condition in which the synapses are operating in a partially depleted state. Some 
variations on the phenomenological models attempt to include use-dependency explicitly by introducing variables that mimic vesicle refractory and release-ready states, but these models do not generalize particularly well either [Tsodyks et al, 1998; Dittman et al, 2000].

Another class of STP model characterizes the observed short term dynamics with a series of response surfaces derived via the Volterra series expansion [Song et al, 2009]. While the Volterra series expansion for synaptic dynamics is in principle complete, it is also cumbersome, since the history dependency extends potentially dozens of spikes back in time. The complete characterization of synaptic dynamics by Volterra series requires the determination of as many nonlinear kernels as there are points in the history. Since it's not practical to go beyond about five kernels, this method is seldom used. Furthermore, it shares with other phenomenological approaches an indirect connection between experimentally observable manipulations and model parameters. The Volterra series expansion does however provide a formalism that has been used to prove the general nonlinear filtering properties of synaptic dynamics [Maass and Son$\operatorname{tag}, 2000]$.

\subsubsection{Phenomenological models}

Despite the lack of predictive power, the phenomenological approach is relatively simple, so it has utility in large-scale computational models. Generalization of a phenomenological model for descriptive purposes would imply that the form of the model would suffice to simulate the behavior of the synapses in the same conditions used to 
develop the model. There would not, however, be any direct connections between model parameters and physiological parameters. With this understanding, the phenomenological approach was applied to the Frerking data.

\section{The Varela model}

The simplest phenomenological model [Varela et al, 1997], was applied first. In the Varela model, the gain of the $f$ EPSP is simulated by the product of independent facilitative $(F)$, and depressive $(D)$ terms,

$$
G_{n, m}(t, \alpha)=\prod_{i=1}^{n} F_{i}\left(t, \alpha_{i}\right) \prod_{j=1}^{m} D_{j}\left(t, \alpha_{j}\right),
$$

where $n$ is the number of facilitory terms, $m$ is the number of depressive terms, $G_{n, m}(t, \alpha)$ is the simulation at time $t$ from the model having $n$ facilitory and $m$ depressive terms, and $\alpha$ is the vector of model parameters. Each $\alpha_{i}$ and $\alpha_{j}$ has two components, an update value and a time constant, for example, $\alpha_{i}=\left\{\Delta_{\mathrm{up}, F_{i}}, \tau_{F_{i}}\right\}$. The $\alpha$ vector is the concatenation of all $\alpha_{i}$ and $\alpha_{j}$. The meaning of the parameters is explained in Eqns.(2.3) and (2.4). The parameters in $\alpha$ were randomly initialized from uniform distributions in sets of 1000. Variations of Eqn. (2.1) were applied to the fEPSP data. All possible combinations of $\{n \mid n \in \mathbb{Z}, 1 \leq n \leq 5\}$ and $\{m \mid m \in \mathbb{Z}, 1 \leq m \leq 5\}$ were tried, using sequential quadratic programming to optimize the fit of the model to the data. The criteria function for the optimization routine was the sum of the 2-norm of the difference between the model and the data: 


$$
\underset{\alpha \in \mathbb{R}^{2(n+m)}}{\min (f(t, \alpha))}=\sum_{j=1}^{M} \sum_{i=1}^{N_{j}}\left\|\left(G_{n, m}\left(t_{i}, \alpha\right)-\hat{G}_{j}\left(t_{i}\right)\right)\right\|
$$

where $M$ is the number of individual data sets, $N_{j}$ the number of points in the $j^{\text {th }}$ data set, and $\hat{G}_{j}\left(t_{i}\right)$ the data value in set $j$ at $t_{i}$.

The terms in the Varela model are described by first order O.D.E.s with instantaneous forcing functions applied at the spike arrival times. For example, the facilitory terms are described by:

$$
\frac{d}{d t} F_{i}(t)=\frac{1-F_{i}(t)}{\tau_{F_{i}}}+\Delta_{\mathrm{up}, F_{i}} \sum_{k=1}^{N} \delta\left(t-t_{k}\right), \quad \Delta_{\mathrm{up}, F_{i}} \in\left[0, \Delta_{\max , i}\right]
$$

where $\tau_{F_{i}}$ is the decay time constant of the $i^{\text {th }}$ facilitory term, $\delta\left(t-t_{k}\right)$ is the Dirac delta function applied at the $k^{\text {th }}$ spike arrival, and $\Delta_{\mathrm{up}, F_{i}}$ is the update applied at each arriving spike. Equation (2.3) has a convenient solution in terms of the Heaviside function, $\Theta\left(t-t_{k}\right)$

$$
F_{i}(t)=1+\Delta_{\text {up }, F_{i}} \sum_{k=1}^{N} e^{\frac{-\left(t-t_{k}\right)}{\tau_{F_{i}}}} \Theta\left(t-t_{k}\right)
$$

Thus, for facilitation, all the responses can be evaluated simultaneously. If there is an imposed limit on the amount of facilitation that can accumulate, then the updates must be individually determined with each spike arrival. The depressive terms are similar, but the updating is multiplicative:

$$
D_{j}(t)=\prod_{k=1}^{N}\left(1-\Delta_{\mathrm{up}, D_{j}} e^{\frac{-\left(t-t_{k}\right)}{\tau_{D_{j}}}} \Theta\left(t-t_{k}\right)\right), \quad \Delta_{\mathrm{up}, D_{j}} \in[-1,0] .
$$


It was found that the Varela model could adequately describe the ordered or the complex data separately, but not with the same parameters, thus failing to generalize. The discrepancy between the models was most apparent at the shortest ISIs, where the complex trains show large instantaneous depression, while the ordered trains show gradual depression following some amount of rapid facilitation. Models that were fit to the ordered trains facilitated on most of the short ISIs in the complex data. No combination of parameters, models, or training regimes, including simultaneous training on ordered and complex data, could overcome this discrepancy. Therefore it was determined that the Varela model can describe a specific activity regime, but cannot generalize to arbitrary ISI trains.

Figures 2.3-2.6 illustrate the behavior of a Varela model with two facilitative and three depressive terms, which was the minimal configuration found to adequately describe either the ordered or the complex Frerking data. Figure 2.3 shows the success of the model in fitting the ordered data. Figure 2.4 shows the failure of the same model in predicting the complex data. Table 2.1 lists the parameters of the model. Figure 2.5 shows the success of the same form of model (2F3D) fitted to the complex data, and Fig. 2.6 shows the failure of the same model to predict the ordered data. Table 2.2 lists the parameters from this model. 

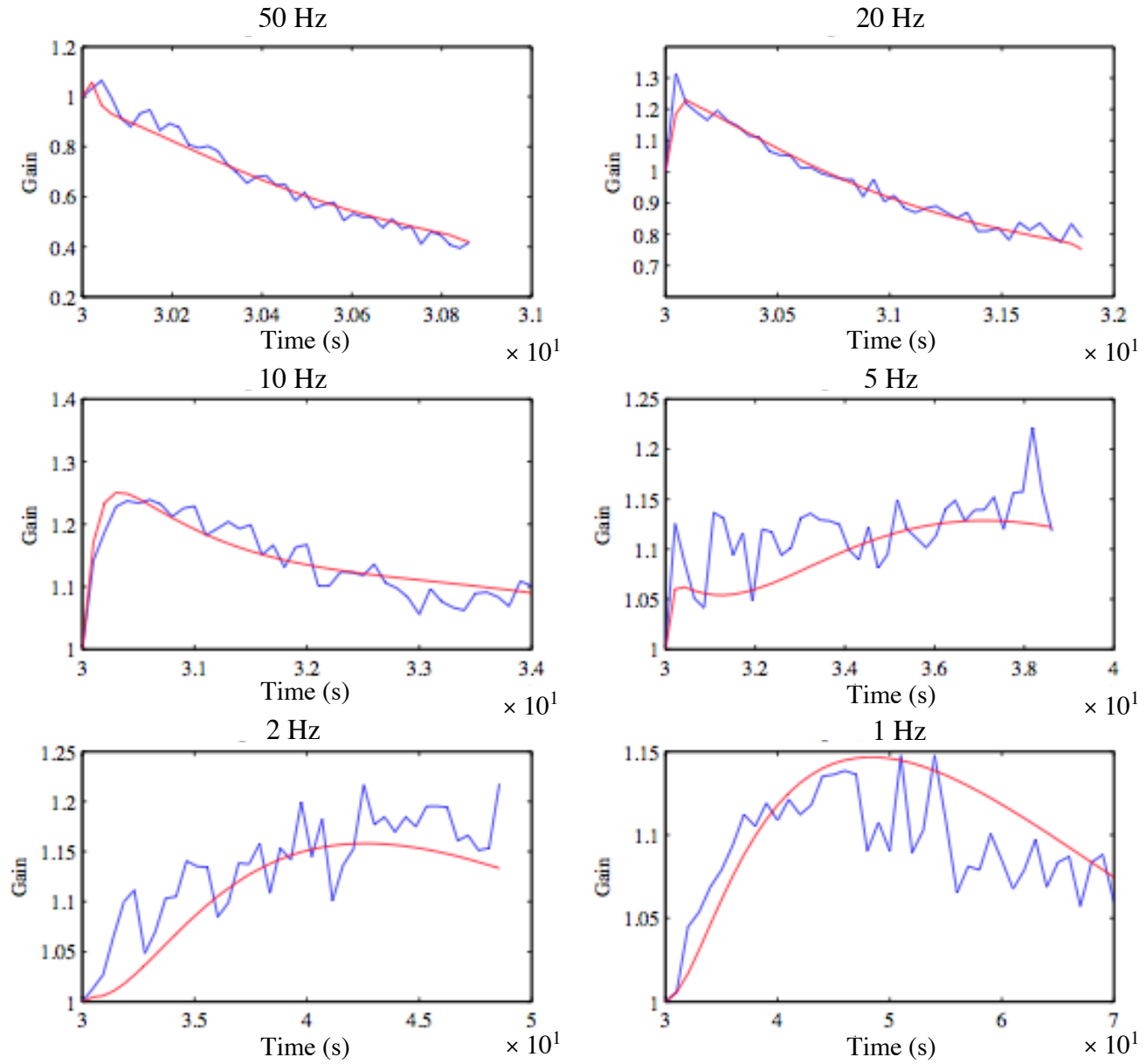

Figure 2.3. A descriptive first-order model having two facilitory and three depressive terms (2F3D) trained on ordered data (Table 2.1). 

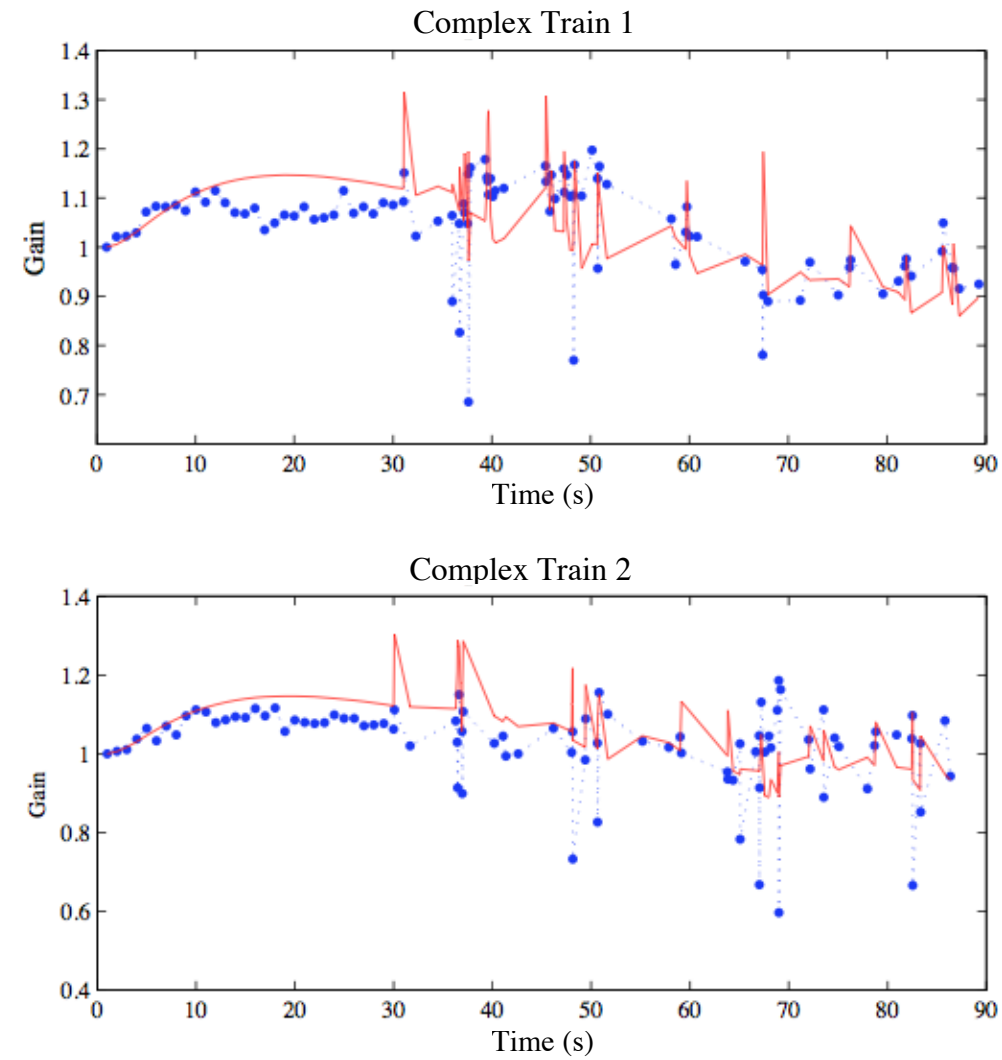

Figure 2.4. The model shown in Fig. 2.3 applied to the complex data (Table 2.1). The failure most clearly occurs at the shortest ISIs, where the model predicts a strong facilitation, but the data shows a strong depression.

Table 2.1. Parameters of the 2F3D model trained on ordered data (Figs. 2.3, 2.4).

\begin{tabular}{|c|c|c|}
\hline & $\tau(\mathrm{ms})$ & update \\
\hline $\mathrm{F}_{1}$ & 77 & 1.7487 \\
\hline $\mathrm{F}_{2}$ & 6701 & 0.0886 \\
\hline $\mathrm{D}_{1}$ & 35 & 0.1543 \\
\hline $\mathrm{D}_{2}$ & 3644 & 0.9394 \\
\hline $\mathrm{D}_{3}$ & 33284 & 0.9923 \\
\hline
\end{tabular}



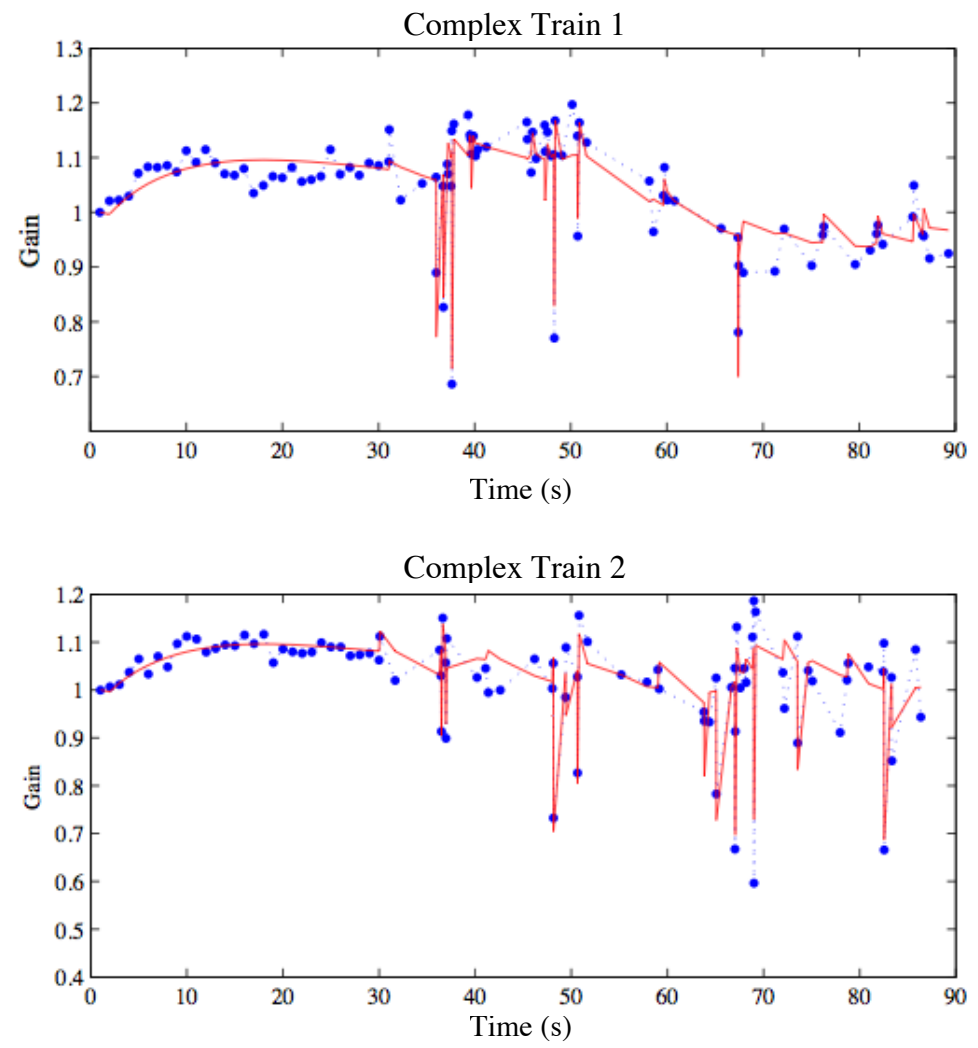

Figure 2.5. A descriptive first-order model having 2 facilitory and 3 depressive terms (2F3D) trained on complex data (Table 2.2). Here the model appears to capture the use-dependent depression.

Table 2.2. Parameters of the 2F3D model trained on complex data (Figs. 2.5, 2.6).

\begin{tabular}{|c|c|c|}
\hline & $\tau(\mathrm{ms})$ & update \\
\hline $\mathrm{F}_{1}$ & 75 & 2.3542 \\
\hline $\mathrm{F}_{2}$ & 12778 & 0.0269 \\
\hline $\mathrm{D}_{1}$ & 92 & 0.1037 \\
\hline $\mathrm{D}_{2}$ & 3287 & 0.997 \\
\hline $\mathrm{D}_{3}$ & 43971 & 0.9917 \\
\hline
\end{tabular}



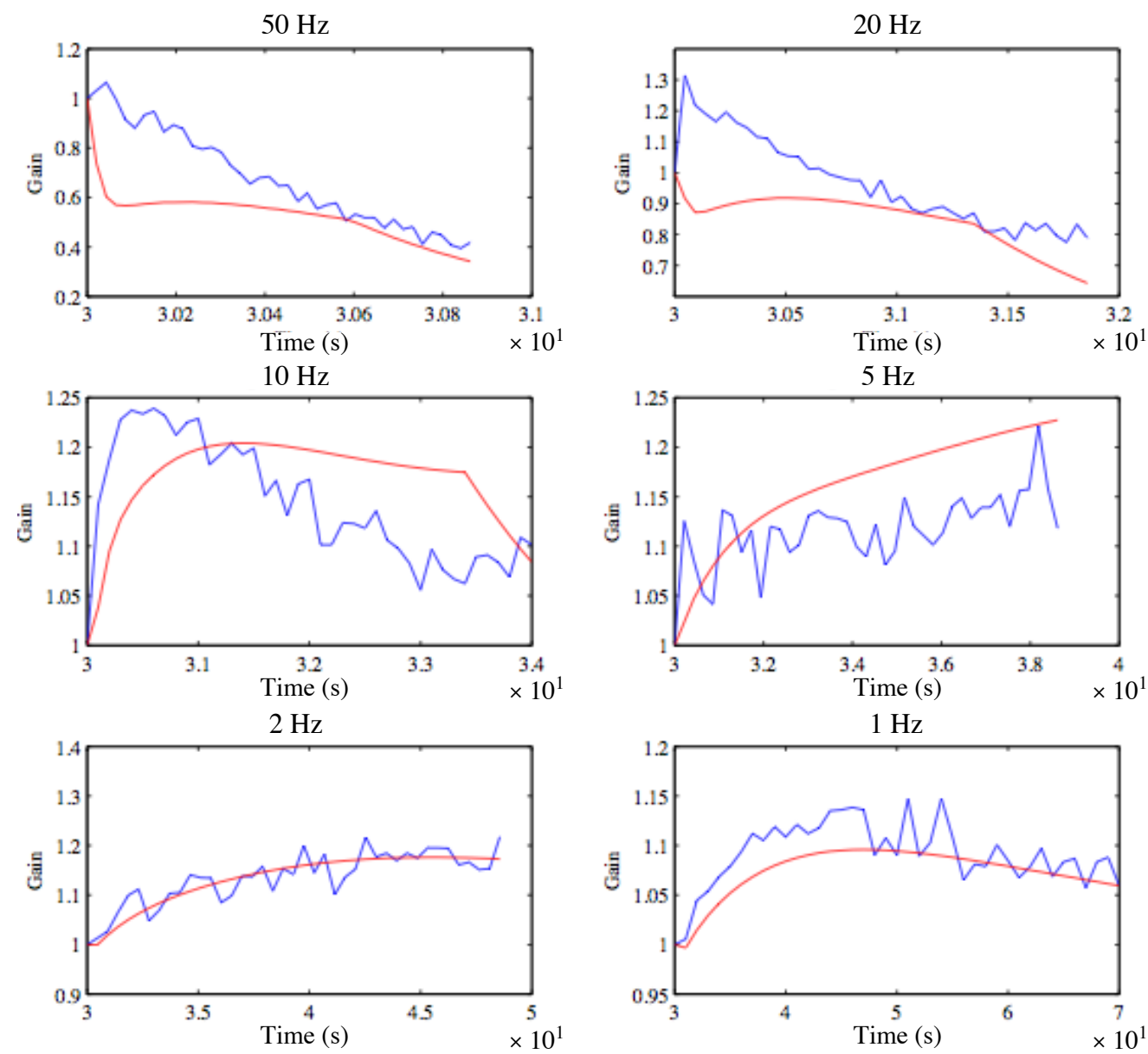

Figure 2.6. The model shown in Fig. 2.5 applied to ordered data (Table 2.2). The model fails to predict high-frequency behavior.

\section{The Dittman model}

Phenomenological models that attempt to describe use-dependency, as exemplified by Dittman [Dittman et al, 2000] and extended by Dobrunz [Sun et al, 2005] have the general form, 


$$
G(t)=\alpha N_{T} F(t) D(t)
$$

where $\alpha=$ mean $f$ EPSC amplitude, $N_{T}=$ number of release sites, and $F(t) D(t)=$ probability of release $\in[0,1]$. The most prevalent process in the presynaptic terminal is the influx and reuptake of calcium. The facilitory and depressive terms in the Dittman model indirectly model the residual calcium concentration by considering the concentration of undetermined calcium-bound molecules, $\mathrm{CaX}_{F}$ and $\mathrm{CaX}_{D}$. Since these proposed molecules do not directly represent calcium levels, and are not attributed to any specific process, the model amounts to essentially a reprisal of the Varela model, except that the $F$ and $D$ terms are scaled to the interval $[0,1]$ and the $D$ term is described by a nonlinear ODE that accelerates recovery. The rescaling allows the expression for $G(t)$ in Eqn. (2.6) to be compared to the form of Eqn. (2.1). Both of the CaX dynamic equations are first-order:

$$
\frac{d C a X}{d t}=\frac{-C a X(t)}{\tau}+\Delta \delta\left(t-t_{0}\right)
$$

The rescaling of $\mathrm{CaX}_{F}$ to arrive at $F$ is given by:

$$
F(t)=F_{1}+\frac{1-F_{1}}{1+K_{F} / \operatorname{CaX}_{F}(t)},
$$

where $K_{F}$ is the dissociation constant of the calcium-bound molecule, $\mathrm{CaX}_{F}$. The $D$ term is modified to include $\mathrm{CaX}_{D}(t)$ dependent recovery,

$$
\frac{d D(t)}{d t}=(1-D(t)) k_{\mathrm{recov}}\left(\operatorname{CaX}_{D}(t)\right)-D(t) F(t) \Delta_{D} \delta\left(t-t_{0}\right),
$$

where $k_{\text {recov }}\left(\mathrm{CaX}_{D}\right)$ is given by: 


$$
k_{\text {recov }}\left(\operatorname{CaX}_{D}(t)\right)=k_{0}+\frac{k_{\max }-k_{0}}{1+K_{D} / \operatorname{CaX}_{D}(t)} .
$$

The success of the Dittman model at fitting the Frerking data set was on par with a 1F2D Varela model. The discrepancy between the short ISI responses in the complex trains and the short ISIs in the ordered trains persisted.

\section{Other phenomenological models}

Variations and elaborations of the semi-mechanistic use-dependent models exist. The more successful such models include the Markram [Tsodyks et al, 1998], Dobrunz [Sun et al, 2005], Klyachko [Kandaswamy et al, 2010], and McRae [Lee (CCJ) et al, 2009] models.

The Markram model [Tsodyks et al, 1998] tracks the fractional states of the synapse in the cycle of vesicle activity that switches from release-ready, $x(t)$, to releasing, $y(t)$, to refractory, $z(t)$, then to release-ready again:

$$
\begin{gathered}
\frac{d x}{d t}=\frac{z(t)}{\tau_{\text {rec }}}-P(t) x(t) \delta\left(t-t_{\mathrm{ap}}\right) \\
\frac{d y}{d t}=P(t) x(t) \delta\left(t-t_{\mathrm{ap}}\right)-\frac{y(t)}{\tau_{\mathrm{in}}} \\
\frac{d z}{d t}=\frac{y(t)}{\tau_{\text {in }}}-\frac{z(t)}{\tau_{\mathrm{rec}}},
\end{gathered}
$$

where $P(t)$ is the average release probability, $\tau_{\text {in }}$ is the time constant of entry into the refractory state, $\tau_{\text {rec }}$ is the time constant of recovery to the release-ready state, and $t_{\text {ap }}$ is the time of occurrence of action potentials. This model was not applied to the Frerking data, and its published results do not generalize well [Tsodyks and Markram, 1997]. 
The Dobrunz model [Sun et al, 2005] includes Dittman's pseudo-calcium dependency and Markram's tracking of the states of the synapse, but describes the release probability in terms of the average number of readily releasable (RRP) vesicles, $n(t)$ :

$$
P(t)=1-(1-\alpha(t))^{n(t)},
$$

where $\alpha(t)$ is the average individual vesicle release probability. The individual vesicle release probability depends on the equilibrium occupancy of the release site by a calcium-bound molecule, $\mathrm{CaX}_{F}$ :

$$
\alpha(t)=\alpha_{1}+\frac{1-\alpha_{1}}{1+K_{F} / \mathrm{CaX}_{F}(t)} .
$$

The size of the readily releasable pool (RRP), $n(t)$, decreases after each spike by an amount equal to the average release (the release probability per active synapse multiplied by the fraction of active synapses). The empty sites are refilled to the maximum size, $n_{T}$, at a constant rate, $R$, and $n(t)$ is given by:

$$
\frac{d n}{d t}=-P(t) x(t) \delta\left(t-t_{\mathrm{ap}}\right)+\left(n_{T}-n(t)\right) R .
$$

The final conceptual contribution made by the Dobrunz model is an indirect calcium dependency on the recovery rate of synapses to the release-ready state, $\tau_{\text {rec }}$, in the $x(t)$ equation in Eqns.(2.11):

$$
\frac{1}{\tau_{\text {rec }}}=k_{\text {rec }}=k_{0}+\frac{k_{\max }-k_{0}}{1+K_{D} / \operatorname{CaX}_{D}(t)} .
$$


Despite these reasonable changes to the framework laid by Dittman and Markram, the Dobrunz model also has trouble producing a unified description of more than one set of constant ISI data [Sun et al, 2005]. The time constant for the $\mathrm{CaX}_{F}$ equation has to be adjusted in order to describe the experimental data having 2, 5, and more (steady state) constant rate stimuli.

The Klyachko model [Kandaswamy et al, 2010] relates three instantaneously updating and exponentially decaying facilitative terms, $\varphi_{1}, \varphi_{2}$, and $\alpha$, to the vesicle release probability, $\pi$, through Dodge-Rahamimoff relations [Dodge and Rahamimoff, 1967]:

$$
\pi=\lambda\left(\frac{\varphi_{1}}{1+\eta_{1} \varphi_{1}}+1\right)\left(\frac{\varphi_{2}}{1+\eta_{2} \varphi_{2}}+1\right)\left(\frac{\alpha}{1+\mu \alpha}+1\right),
$$

where $\lambda, \eta$, and $\mu$ are constants. Depression was described as a two-pool vesicle depletion process involving vesicle release from a finite readily releasable pool, and refilling from a larger recycling pool, and an infinite reserve pool. The recycling pool was also refilled from the reserve pool. Thus, there were three refilling rates that were kept constant, rather than given a calcium-dependent acceleration. Overall synaptic release was defined as: $P_{\text {syn }}=1-(1-\pi)^{n}$, where $n$ is the number of vesicles in the readily releasable pool, which was tracked according to the two-pool depletion model. The initial number of vesicle was set to 8 in the readily releasable pool, and to 17 in the recycling pool. The facilitative parameters and vesicle pool refilling rates were obtained by fitting to data from constant-rate trains.

The Klyachko model is remarkable because it fit natural trains quite well $\left(r^{2}=0.774\right)$ using the parameters obtained from fitting to constant-rate data. However, 
the published results didn't include any data that had gain values less than one.

The McRae model [Lee (CCJ) et al, 2009] comes the closest to a mechanistic model among the phenomenological models mentioned here. Calcium entry and decay is modeled as a first order process:

$$
\tau_{\mathrm{Ca}} \frac{d C a(t)}{d t}=\left(-C a+C a_{0}\right)+K_{\mathrm{Ca}} I_{\mathrm{Ca}} \approx\left(-C a+C a_{0}\right)+K_{\mathrm{Ca}} \sum_{k=1}^{M} \delta\left(t-t_{k}\right),
$$

where $K_{\mathrm{Ca}}$ is a constant gain value that converts calcium current into the unit of concentration, and $\delta(\cdot)$ is the Dirac delta function. The rate of neurotransmitter release, $N T(t)$, is then related to the number of releasable vesicles, $N_{r e l}$, the probability of release, $P_{r e l}$, and the calcium current, $I_{\mathrm{Ca}}$, by:

$$
\frac{d N T(t)}{d t}=n N_{r e l} P_{r e l} I_{\mathrm{Ca}}=n N_{\text {total }} R_{\text {rel }} P_{\text {rel }} I_{\mathrm{Ca}},
$$

where $n$ is a stoichiometric coefficient, $R_{r e l}$ is the ratio of releasable vesicles, and $N_{\text {total }}$ is the sum of empty and releasable vesicles. During stimulation, $R_{r e l}$ depends on the difference between the rate of recovery, $k_{\text {recov }}$, and the rate of exocytosis, $P_{r e l} I_{\mathrm{Ca}}$ :

$$
\frac{d R_{r e l}(t)}{d t}=k_{\text {recov }}\left(1-R_{r e l}\right)-P_{r e l} I_{\mathrm{Ca}} R_{r e l} .
$$

The McRae model then relates $P_{r e l}$ to the calcium concentration through the Hill equation (see Appendix B):

$$
P_{\text {rel }}=P_{\text {rel,max }} \frac{C a^{n_{\text {Hill }}}}{C a^{n_{\text {Hill }}}+K_{\text {rel, }, 1 / 2}^{n_{\text {Hill }}}},
$$

where $n_{\text {Hill }}$ is the cooperativity of the reaction, and $K_{r e l, 1 / 2}$ is the calcium concentration at half max occupancy. The value of $n_{\text {Hill }}$ was set to four. The McRae model also 
includes a similar term describing asynchronous release, that has a lower cooperativity. The asynchronous term is added to the synchronous release term. Finally, the McRae model includes a calcium dependent increase to the rate of recovery, $k_{\text {recov }}$ :

$$
k_{\text {recov }}=k_{\text {recov }, 0}+\left(k_{\text {recov }, \max }-k_{\text {recov }, 0}\right) \frac{C a}{C a+K_{\text {recov }, 1 / 2}} .
$$

The McRae model was shown to describe both facilitative and depressive processes that occurred independently in parallel fiber and calyx of Held synapses, and concurrently in neocortical pyramidal cell synapses. However, the model was not demonstrated on non-constant trains. Nevertheless, the McRae model provides valuable insights into describing calcium-dependent processes, including the non-constant recovery rate of releasable vesicles.

The Markram, Dobrunz, Klyachko, and McRae models were not applied to the Frerking data. However, the Klyachko paper, had an intriguing suggestion for the formulation of vesicle release in terms of the energetic barrier to vesicle fusion in the presence of calcium, $U_{\mathrm{Ca}}$, through the vesicle fusion rate, $\rho$, and the duration of calcium action, $\tau_{\mathrm{Ca}}$, according to the relation, $\pi=\rho \tau_{\mathrm{Ca}}=\gamma \exp \left(-U_{\mathrm{Ca}} / k T\right)$, where $k$ is the Boltzmann constant, $T$ the temperature, and $\gamma$ a proportionality constant. The energy barrier was suggested to be reduced in the presence of calcium due to the action of synaptotagmin. While these suggestions were not implemented in the Klyachko model, they did inspire the treatment of vesicle fusion in the DSCI model. 


\section{Summary of the phenomenological modeling approach}

The form of the phenomenological model roughly indicates the number and type of processes (facilitative, $F$, or depressive, $D$ ) that are required to explain the Frerking STP data, as well as the primary behavior that remains to be explained by a mechanistic model, namely the rapidly acting and rapidly recovering use-dependent depression seen in the complex data. Although the exponential processes probably indicate the rough form and number of processes required, there need not be a direct correlation between $F$ and $D$ processes and synaptic mechanisms.

The limited success of the phenomenological models suggests that another modeling approach be taken. The mechanistic approach is advantageous because model parameters can be bounded according to experimental observations, and the direct correspondence between parameters and mechanisms allows comparison between experimental manipulation and modeling simulations. The correspondence between parameters and observables means that a mechanistic model should have predictive power under conditions where synaptic processes are altered, which could guide experiments.

\subsubsection{Mechanistic models}

There has been really only one notable attempt to simultaneously explain facilitive and depressive synaptic dynamics mechanistically [Pan and Zucker, 2009]. In their paper, Pan and Zucker describe mechanisms that have been unable to describe both behaviors in previous models, including heterogeneity of active zone parameters (size, number and density) [Bradacs et al, 1997; King et al, 1996], "metabolic activity" 
[Nguyen and Kandel, 1997], calcium entry or local concentration during an action potential [Msghina et al, 1997], and the size of the readily releasable pool of vesicles [Millar et al, 2002].

\section{The Zucker model}

The Zucker model describes short-term time-resolved neurotransmitter quantal release dynamics of both phasic and tonic crayfish neuromescular junctions (NMJs). Phasic NMJs display depressive behavior, while tonic NMJs display facilitative behavior. Since presynaptic processes are largely similar between crayfish neuromuscular junctions and vertebrate central synapses [Atwood and Karunanithi, 2002; Zucker et al, 1999], the types of mechanisms that would be needed in order to describe mammalian synapses should be similar to, or at least include, the processes described by Zucker.

The Zucker model uses a sequential two-pool vesicle depletion model with calciumdependent mobilization of vesicles from a lower release probability pool to a higher release probability pool. The influx of calcium is based on a Hodgekin/Huxley model of invertebrate P/Q calcium channels [Llinas, 1999]. Spatial diffusion of calcium ions is realistically modeled to simulate positional priming of vesicles near calcium channels. Fast and slow calcium buffers are included in the model. A 12-state kinetic model of synaptotagmin calcium binding with distinct calcium binding domains (C2A and C2B) is included. Finally, release site refractoriness (the short period of time following vesicle fusion during which subsequent fusion events do not or cannot occur) is modeled. 
The Zucker model has 28 parameters, most of which were constrained by physiologically observed values, but several of which were allowed to vary during fitting. Among those allowed to vary were the sizes of the two primed vesicle pools. In order to account for the behavior seen at tonic synapses, the estimation of the size of the primed vesicle pool had to be much less than a single vesicle, which may be an unrealistic result. However, the amount of facilitation seen at crayish tonic NMJs is huge ( $1000 \%$, so the initial value of releasable vesicles might have to very small.

The Zucker model was able to describe several features of synaptic behavior, including the recovery of transmission following readily releasable vesicle depletion, the effect of endogenous and exogenous calcium buffers (including fura-2 and diazo-2), and to a limited extent, the kinetics of transmitter release and synaptic plasticity. The main problem with the Zucker model is that it doesn't simultaneously reproduce the behavior of the phasic and the tonic synapses using the same set of parameter values. While these synapses are distinct in the crayfish, the lack of a simultaneous fit indicates that the interaction of facilitative and depressive effects probably eludes the Zucker model, despite the attention to detail. Zucker also did not publish results of his model with variable interval stimulus trains, so the generalization of the model is unknown. The Zucker model was not applied to the Frerking data.

\section{Summary of the mechanistic modeling approach}

The complexity of the Zucker model prevents it from being useful in a computational setting, where processing efficiency is required. For this purpose, an efficient 
model is needed that comes from a simpler perspective. This means making some decisions about the importance of modeling the diffusion of calcium, for instance, or including a complete description of synaptotagmin calcium-binding kinetics.

The following section describes several mechanisms that are operative at Schaffer collateral (SC) presynaptic terminals, and introduces a new biophysical model of the SC short term dynamics based upon those mechanisms.

\subsection{New biophysical model of STP}

The presynaptic mechanisms approximated in the dual sensor competitive interaction (DSCI) model of STP are introduced here, along with supporting evidence for the mechanisms. The model equations are compiled in Appendix B.

\subsubsection{Postsynaptic processes}

Postsynaptic processes are not thought to exhibit influence over short term synaptic plasticity in the hippocampus [Atluri and Regehr, 1996; Hashimoto and Kano, 1998; Dobrunz and Stevens, 1999; Hanse and Gustafson, 2001; Zucker and Regehr, 2002]. Nevertheless, postsynaptic processes, including receptor saturation and desensitization [Xu-Friedman and Regehr, 2004], and retrograde signaling [Davis and Murphey, 1994] could, in principle, influence synaptic dynamics. Receptor saturation and desensitization would both operate as depressive factors [Trussel et al, 1993], whereas retrograde signaling could be either facilitative or depressive [Akopian and Walsh, 2002; Wilson and Nicoll, 2002]. Postsynaptic receptors in the CA1 have been found not to desensi- 
tize under paired pulse facilitation [Hjelmstad et al, 1999], although intervals only as short as $30 \mathrm{msec}$ were reported. Despite this caveat, the DSCI model of STP does not include postsynaptic saturation or desensitization. Nor does the DSCI model include any dependence on the state of postsynaptic LTP, which has been shown to effect presynaptic release probability, possibly through retrograde signaling [Liebold and Bendels, 2009; Regehr et al, 2009].

\subsubsection{Presynaptic processes}

Of the myriad presynaptic processes that affect neurotransmitter release [Stevens, 2003; Sudhof, 2004; Catterall and Few, 2008; Davies and Zamponi, 2008; Rizo and Rosenmund, 2008], the DSCI model invokes only a subset, and mainly those directly involving the release machinery. The DSCI model rests upon the following observations and assumptions:

1) Observation: Synchronous exocytosis depends cooperatively on calcium through a catalytic intermediary identified as synaptotagmin [Rusakov, 2006].

2) Observation: Synaptotagmin (specifically isoform syt1 in the hippocampus [Xue et al, 2010]) can trigger neurotransmitter release at high calcium concentration levels, but suppress release at low levels.

3) Assumption: Intermediate states of the kinetics that cooperatively bind calcium are short-lived enough to approach equilibrium on the time scale of the changes in calcium concentration that occur between spikes [Chapman, 2008; Lee (CCJ) et al, 2009]. 
4) Observation: Synaptotagmin contributes energy to exocytosis through a calciumdependent interaction with a membrane-bound release mechanism, identified as the SNARE complex [Stewart et al, 2000; Jahn et al, 2003; Gaffaney et al, 2008].

5) Observation: At least two isoforms of synaptotagmin, each having unique calcium-binding kinetics, and each contributing a unique amount of energy to exocytosis, compete for one binding site at each SNARE complex [Bhalla et al, 2005; Sun et al, 2007].

6) Observation: There are at least three SNARE complexes associated with each releasable vesicle [Hua and Scheller, 2001; Mohrmann et al, 2010].

7) Observation: Releasable vesicles exist in two distinct populations defined by the rates at which vesicles are replaced, and potentially, but not necessarily, by vesicle proximity to calcium channels [Sudhof, 2004].

8) Assumption: There is a single type of voltage-gated calcium channel (this assumption is unequivocally wrong [Jarvis and Zamponi, 2005], but justifiable because calcium entry kinetics are modeled as instantaneous processes, and no calcium channels blockers were applied during data collection).

9) Assumption: Vesicular release is uniquantal [Hanse and Gustafson, 2001; Stevens, 2003; Nadkarni et al, 2010].

10) Assumption: Residual calcium concentration can be described as homogenous within a single compartment [Neher and Augustine, 1992; Tank et al, 1995; Lee (CCJ) et al, 2009]. 
11) Assumption: The number of vesicles in the readily-releasable pool does not exceed the resting population value [Lee (CCJ) et al, 2009].

12) Assumption: There is no trafficking between willing and reluctant pools (probably not true: see [Lee (JS) et al, 2012]).

The novelty of the DSCI model lies in the description of simultaneous facilitative and depressive components of STP based on a competitive interaction between two unique calcium sensors. The DSCI model is noteworthy because the simultaneous description of short-term facilitation and depression in complex trains has eluded mechanistic models of STP [Lee (CCJ) et al, 2009; Pan and Zucker, 2009], and because the DSCI model unifies both processes using an emerging paradigm of neurotransmitter release involving dual calcium sensors [Sun et al, 2007]. Due to the conserved nature of the calcium sensors, the model may have applicability at other CNS synapses. The model is also extensible, so that multiple types of calcium channels, mobility between vesicle pools, and other molecular contributions to vesicle priming could be included.

\subsubsection{Release rates, vesicle pools, and calcium sensors}

Presynaptic processes that affect synaptic dynamics ultimately act upon the rates of exocytosis, endocytosis, and refilling of neurotransmitter-containing presynaptic vesicles [Sudhof, 2004; Lee (CCJ) et al, 2009]. However, attributing specific mechanisms to any of these vesicle cycling processes is complicated by the observation of simultane- 
ous heterogenous neurotransmitter (NT) release rates [Walter et al, 2011]. To simplify matters, neurotransmitter release rates can be attributed to vesicle release rates through the existence of unique populations of vesicles at the active zone and through variations in the molecular composition of the release mechanism [ibid]. The interaction between these components of vesicle release is still unknown though, so both components are included in the DSCI model, and introduced here, starting with the identity and composition of the vesicle pools.

Vesicles that are docked at the active zone in the presynaptic terminal exist in readily-releasable and reserve states, or "pools", as determined by release rate (which is directly proportional to release probability) [Sudhof, 2004]. In the hippocampus, the readily-releasable pool (RRP) has on average between 2 and 12 vesicles [Harris and Sultan, 1995]. At the larger calyx of Held synapse, located in the auditory brainstem, the readily releasable pool of vesicles can be further divided into immediately-releasing, and reluctantly-releasing vesicles [Sakaba and Neher, 2001]. Immediatelyreleasable vesicles (referred to here as "willing" vesicles) are able to exocytose synchronously with the spike-induced membrane depolarization. The willing pool is small and depletes quickly during repeated stimulation, but can be rapidly replenished from docked and undocked populations of vesicles [Hosoi et al, 2007] (although see [Gabriel et al, 2011]). The reluctant vesicle pool requires greater stimulation for synchronous release, but paradoxically is responsible for asynchronous release that occurs at residual calcium concentrations [Sakaba, 2006]. 
There are two hypotheses concerning the composition of docked vesicles. The older, generally accepted view, known as positional priming, characterizes the readilyreleasable pool in terms of vesicle proximity to voltage-gated calcium channels (VGCCs) [Neher and Sakaba, 2008]. According to the positional priming hypothesis, voltage-gated calcium channels, and the vesicle release machinery (the SNARE complexes), are dissociated from each other in the active zone. The positional priming hypothesis predicts that reluctant vesicles are converted into willing vesicles by a process that moves the reluctant vesicles closer to the calcium channels (or vice-versa).

Support for the positional priming hypothesis comes from the observation that a mutation in synaptotagmin that disrupts the binding of synaptotagmin with the SNARE complex, also disrupts the synchrony between action potentials and NT release, while destabilizing (reducing) the RRP, yet leaving the calcium sensitivity of exocytosis intact [Young and Neher, 2009]. Evidence also exists for an actin-dependent process that dynamically resizes the reluctant and willing pools while conserving the total RRP population [Lee (JS) et al, 2012].

The DSCI model approximates positional priming by distinguishing willing and reluctant vesicle pools according to their degree of exposure to the maximum calcium concentration near the VGCCs. Willing vesicles experience the full concentration, while reluctant vesicles see a fixed fractional amount of the full concentration (see section 2.4.4). The numbers of readily releasable vesicles in the willing and reluctant pools at rest (in the absence of spikes) are given as fractions, $\phi_{n}$ : $\left(0 \leq \phi_{n} \leq 1\right)$, of the 
total number, $n_{\text {rest }}$, of readily releasable vesicles in the presynaptic terminal at rest: $n_{\text {rest,willing }}=n_{\text {rest }} \times \phi_{n}$, and $n_{\text {rest,reluctant }}=n_{\text {rest }} \times\left(1-\phi_{n}\right)$. Vesicles are not exchanged between pools in the DSCI model, so $\phi_{n}$ is constant. The maximum number of vesicles is limited to the resting value.

According to another hypotheses concerning the composition of docked vesicles, the SNARE complex is tethered to one or two VGCCs, forming an excitosome [Atlas, 2010]. This hypothesis, which has been called the molecular priming hypothesis [Lee (JS) et al, 2012], incorporates the other component of presynaptic vesicle release that can be interpreted in terms of NT release rate, namely, variations in the composition of the release mechanism. In the molecular priming hypothesis, docked vesicles are bound to excitosomes in a calcium-independent process, but the willing vesicles (referred to as "releasable" vesicles in [Atlas, 2010]) undergo an additional calciumdependent priming process that puts them in a state that is more energetically favorable for exocytosis. The vesicle priming process is attributed to a conformational change in specific isoforms of synaptotagmin (syt 1 or syt 2 ), initiated by calcium at one of the two C2 calcium-binding domains (the C2A domain [Shin et al, 2009]). Neurotransmitter release is triggered when the VGCCs open, allowing an influx of calcium that can bind to the remaining $\mathrm{C} 2$ domain (the $\mathrm{C} 2 \mathrm{~B}$ domain), and cause a rapid conformational change that pushes membrane fusion over the final energy barrier. The C2A domain binds three calcium ions, and the C2B domain binds two [Kochubey et al, 2011]. 
Support for the molecular priming hypothesis comes from models of the calcium nanodomain, indicating that the vesicles must be within about $10 \mathrm{~nm}$ of the calcium channels to experience calcium concentrations high enough to engage the release mechanism [Stanley, 1997]. Also, there are subunits of the VGCCs that bind with high affinity to SNARE components [Kim and Catterall, 1997; Cohen and Atlas, 2004], and that if are knocked out, obliterate evoked release [Cohen et al, 2007]. Some results, such as the actin-dependent process discovered by Lee et al [Lee (JS) et al, 2012] can also be interpreted to support the molecular priming hypothesis.

The molecular priming hypothesis is incorporated in the DSCI model by defining individual vesicle release probabilities in terms of the unique calcium binding kinetics of two synaptotagmin isoforms (see section 2.4.7 on the determination of $E_{\text {SNARE }}$ ).

\subsubsection{Extracellular calcium influx and decay}

Divalent calcium cations initiate and regulate many processes in presynaptic terminals [Rusakov, 2006; Neher and Sakaba, 2008; Catterall and Few, 2008; Davies and Zamponi, 2008]. Calcium enters the presynaptic terminal through voltage-gated calcium channels (VGCCs) that are opened by membrane depolarization induced by action potentials. For the duration of the depolarization event ( $\sim 1 \mathrm{~ms})$, VGCCs pump $\mathrm{Ca}^{2+}$ rapidly into the cell, thereby building transient domains of $\left[\mathrm{Ca}^{2+}\right]$, called nanodomains, within about $50-100 \mathrm{~nm}$ of the calcium channels, that can be $25-200 \mu \mathrm{M}$ higher than the average $\left[\mathrm{Ca}^{2+}\right]$ in the bouton [Naraghi and Neher, 1997; Augustine et 
al, 2003]. The steep drop-off in calcium concentration from the nanodomains has also been attributed to rapid calcium-binding processes [Augustine, 2001].

The use of the subscript $\mu$ in the DSCI equations to denote nanodomain $\left[\mathrm{Ca}^{2+}\right]$ is a holdover from the terminology used to describe microdomains, which are transient $\left[\mathrm{Ca}^{2+}\right]$ domains resolved on the scale of the active zone.

\section{Nanodomain calcium}

Calcium influx in response to presynaptic membrane depolarization is approximated in the DSCI model by an instantaneous impulse, $\Delta_{\mathrm{Ca}, \mu}$. This is an acceptable approximation since calcium transients reach peak values within about $500 \mu$ s [Meinrenken et al, 2003], which is less than the width of most spikes, and shorter than any ISI produced by hippocampal pyramidal neurons. The nanodomain calcium concentration, upon the arrival of the $i^{\text {th }}$ spike, is given by:

$$
\left[\mathrm{Ca}^{2+}\right]_{\mu, i}=\left[\mathrm{Ca}^{2+}\right]_{\mathrm{res}, i}+\Delta_{\mathrm{Ca}, \mu},
$$

where $\left[\mathrm{Ca}^{2+}\right]_{\mathrm{res}, i}$ is the mean residual cytosolic calcium concentration evaluated at the time of the $i^{\text {th }}$ spike. Spatially, this expression neglects variations in calcium concentration on the microdomain scale, which would play into the positional priming hypothesis. The DSCI approximates positional priming by limiting the exposure of the reluctantly-releasing vesicle pool to a fixed fraction, $\phi_{\mathrm{Ca}, \mu}$, of the full nanodomain calcium concentration: 


$$
\left[\mathrm{Ca}^{2+}\right]_{\mu, i, \text { reluctant }}=\phi_{\mathrm{Ca}, \mu}\left[\mathrm{Ca}^{2+}\right]_{\mu, i} .
$$

\section{Residual cytosolic calcium}

After the voltage-gated calcium channels close, the nanodomain calcium diffuses into the bulk cytoplasm, where the calcium is buffered, removed, and sequestered [Billups and Forsythe, 2002; Tank et al, 1995]. The residual calcium concentration in the cytoplasm can reach values in the low $\mu \mathrm{M}$ range during repetitive stimulation [Kochubey et al, 2011]. The decay kinetics of the residual calcium concentration can be adequately approximated with a single compartment [Neher and Augustine, 1992], using a bi-exponential function [Koester and Sakmann, 2000]. Furthermore, calcium diffusion from nanodomains occurs rapidly enough to justify an instantaneous approximation of the updates to the two components of the residual calcium concentration [Roberts, 1994; Sinha et al, 1997]. Thus, the DSCI model describes the update and decay of the residual calcium concentration at the time of the $i^{t h}$ spike by:

$$
\left[\mathrm{Ca}^{2+}\right]_{\mathrm{res}, i}=\left[\mathrm{Ca}^{2+}\right]_{\mathrm{res}, 0}+\sum_{k=1}^{i} \Theta\left(t-t_{k-1}\right)\left(\Delta_{\mathrm{Ca}, \text { int }} e^{\frac{-\left(t-t_{k-1}\right)}{\tau_{\mathrm{Caint}}}}+\Delta_{\text {Ca, slow }} e^{\frac{-\left(t-t_{k-1}\right)}{\tau_{\mathrm{Ca}, \mathrm{alow}}}}\right),
$$

where $\Theta(\cdot)$ is the Heaviside function, the subscript, "Ca,int", denotes an intermediate time scale, and the subscript, "Ca,slow", denotes a slow time scale. Decay time constants were determined by Koester and Sakmann to be $552 \mathrm{msec}$ and $40 \mathrm{msec}$. The biexponential expression is the sum of the solutions of two first order linear O.D.E.s, each describing an independent calcium decay process: 


$$
\begin{gathered}
\frac{d}{d t}\left[\mathrm{Ca}^{2+}\right]_{\mathrm{res}, \text { int }}=-\frac{\left[\mathrm{Ca}^{2+}\right]_{\mathrm{res}, \text { int }}}{\tau_{\mathrm{Ca}, \text { int }}}+\Delta_{\mathrm{Ca}, \text { int }} \delta\left(t-t_{\mathrm{spike}}\right) \\
\frac{d}{d t}\left[\mathrm{Ca}^{2+}\right]_{\text {res,slow }}=-\frac{\left[\mathrm{Ca}^{2+}\right]_{\mathrm{res}, \text { int }}}{\tau_{\mathrm{Ca} \text {,slow }}}+\Delta_{\mathrm{Ca}, \text { slow }} \delta\left(t-t_{\mathrm{spike}}\right),
\end{gathered}
$$

where $\delta$ is the Dirac delta function, $\left[\mathrm{Ca}^{2+}\right]_{\text {res }}$ is a function of time, and $t_{\text {spike }}$ is the time of spike arrival at the synaptic bouton.

\subsubsection{Vesicle reloading}

Both the willing and reluctant vesicle pool refilling rates can be described by first order kinetics, however the willing pool refill rate experiences an activity-dependent acceleration [Otsu et al, 2004]. Otsu et al found that the willing pool recovered from depletion with a time constant between about 425 and $840 \mathrm{~ms}$, while the reluctant pool recovered with a time constant between about 15 and 27 seconds. The acceleration of the willing pool refilling rate may be due to recruitment from the reluctant pool [Lee (JS) et al, 2012], or other processes [Sakaba and Neher, 2001]. Recovery has been proposed to be a nonlinearly calcium-dependent process [Dittman, 2000; Hosoi et al, 2007].

The DSCI model replenishes the reluctant vesicle pool at the arrival of the $i^{\text {th }}$ spike by an amount, $n_{\text {refill,reluctant }, i}$, according to:

$$
n_{\text {reluctant }, i}=n_{\text {reluctant }, i-1}+n_{\text {refill,reluctant }, i}
$$

where

$$
n_{\text {refill,reluctant }, i}=\min \left(n_{\text {rest, reluctant }}-n_{\text {reluctant }, i-1}, n_{\text {rest, reluctant }} \eta_{\text {reluctant }, i}\right),
$$


and

$$
\eta_{\text {reluctant }, i}=1-e^{\frac{-\left(t_{i}-t_{i-1}\right)}{\tau_{\text {slow }}}}
$$

Equation (2.29) is the solution of, $\tau_{\text {slow }} \frac{d \eta_{\text {reluctant }}(t)}{d t}=1-\eta_{\text {reluctant }}(t)$, evaluated at $\Delta \mathrm{t}=t_{i}-t_{i-1}$, with $\eta_{\text {reluctant }}(0)=0$. As $\Delta \mathrm{t} \rightarrow 0, \eta_{\text {reluctant }} \rightarrow 0$, and as $\Delta \mathrm{t} \rightarrow \infty, \eta_{\text {reluctant }} \rightarrow 1$. The physical interpretation is that endocytosis, vesicle refilling, and reluctant pool replacement all happen at a rate proportional to the vacancy in the reluctant pool [Trommershauser et al, 2003].

Similarly to the reluctant pool, the willing vesicle pool is updated at the arrival of the $i^{\text {th }}$ spike according to,

$$
n_{\text {willing }, i}=n_{\text {willing }, i-1}+n_{\text {refill,willing }, i}
$$

where

$$
n_{\text {refill,willing }, i}=\min \left(n_{\text {rest,willing }}-n_{\text {willing }, i-1}, n_{\text {rest,willing }} \eta_{\text {willing }, i}\right) \text {. }
$$

The acceleration of the willing pool refilling rate (Eqn. 2.34) can be written as the product of several terms, the first of which is the fractional value:

$$
\tilde{\eta}_{\text {willing }, i}=1-e^{\frac{-\left(t_{i}-t_{i-1}\right)}{\tau_{\text {tast }}}},
$$

obtained from the solution of $\tau_{\text {fast }} \frac{d \tilde{\eta}_{\text {willing }}}{d t}=1-\tilde{\eta}_{\text {willing }}$, evaluated at $\Delta t=t_{i}-t_{i-1}$, with $\tilde{\eta}_{\text {willing }}(0)=0$. The value of $\tilde{\eta}_{\text {willing }, i}$ is weighted by a factor proportional to $\Delta t$ and the residual calcium concentration by the Michaelis-Menton expression:

$$
k_{\text {refill,fast }, i}=\operatorname{Amp}_{\text {refill,fast }} e^{\frac{-\left(t_{i}-t_{i-1}\right)}{\tau_{k, \text { refill }}}}\left(\frac{\left[\mathrm{Ca}^{2+}\right]_{\mathrm{res}, i}}{\left[\mathrm{Ca}^{2+}\right]_{\mathrm{res}, i}+\mathrm{Kd}_{\mathrm{refill}, \mathrm{fast}}}\right),
$$


where Amp refill,fast is a constant, and $\mathrm{Kd}$ is the dissociation constant. The calciumdependent term in Eqn. (2.33) describes the fractional saturation of an unidentified calcium sensor that catalyzes the willing pool acceleration, and that has calcium binding kinetics that greatly exceed the rate at which residual calcium concentraion changes. The exponential term is the solution to the equation, $\tau_{k, \text { refill }} \frac{d}{d t} \tilde{k}(t)=-\tilde{k}(t)$. One possible interpretation of the willing pool reloading process is that a calcium-dependent mechanism provides a transient increase in the willing pool accessibility to resources from the reserve pool. Another interpretation is that reluctant vesicles are briefly converted to willing status by way of another calcium-sensing mechanism, however, this interpretation is incompatible with the DSCI model, since vesicle trafficking between pools is not described.

The final form of the fractional update to the willing pool is the weighted average of $\tilde{\eta}_{\text {willing }, i}$ with $\eta_{\text {reluctant }, i}$ :

$$
\eta_{\text {willing }, i}=\frac{k_{\text {refill,fast }, i} \tilde{\eta}_{\text {willing }, i}+\eta_{\text {reluctant }, i}}{k_{\text {refill,fast }, i}+1} .
$$

In the limit of long ISIs, $k_{\text {refill,fast }} \rightarrow 0$, so $\eta_{\text {willing }} \rightarrow \eta_{\text {reluctant }}$, which decays exponentially at the rate of $\tau_{\text {slow. }}$. However, for ISIs on the same order of magnitude as $\tau_{k \text {,refill }}$, the willing pool receives additional calcium-dependent reloading at the higher rate of $\tau_{\text {fast }}$.

\subsubsection{Quantal release probability}

Vesicle release depends on the probability of the release mechanism overcoming the energy required for lipid bi-layer membrane fusion [Jahn et al, 2003]. In synapses, the 
energy of membrane fusion has been estimated at about $40 k_{B} T$, where $k_{B}$ is the Boltzmann constant and $T$ is the absolute temperature [ $\mathrm{Li}$ et al, 2007]. $P_{\text {ves }}$ can be described in terms of the energy of fusion, $E_{\text {fusion }}$, through the statistical relation:

$$
P_{\text {ves }}\left(E_{\text {fusion }}\right)=\frac{1}{Z} e^{-\beta E_{\text {fusion }}},
$$

where $\beta=\frac{1}{k_{B} T}$. The term $e^{-\beta E_{\text {fusion }}}$ is the Boltzmann factor for the state of vesicle fusion, and $Z$ is the partition function (the sum over accessible states). Although intermediate states exist, synaptic membrane vesicle fusion can be simplified as a two-state system (either the vesicle fuses with the membrane, or it does not). For a two-state system, with a ground state energy, $E_{0}=0$, the partition function is given by:

$$
Z=\sum_{i=0}^{1} e^{-\beta E_{i}}=1+e^{-\beta E_{1}} .
$$

Without assistance, the probability of membrane-vesicle fusion is essentially zero since $E_{1}=E_{\text {fusion }}$. The SNARE complex effectively contributes an energy, $E_{\mathrm{SNARE}}$, towards overcoming the membrane fusion energy barrier. Thus the probability of vesicle fusion can be written in terms of the difference in energies, $E_{\text {diff }}=E_{\text {SNARE }}-E_{\text {fusion }}$ :

$$
P_{\mathrm{ves}}\left(E_{\mathrm{diff}}\right)=\frac{e^{-\beta E_{\mathrm{diff}}}}{1+e^{-\beta E_{\mathrm{diff}}}} .
$$

Note that when $E_{\text {diff }}=0$, this probability is $1 / 2$. However, the probability essentially goes to one when $E_{\mathrm{SNARE}}$ exceeds $E_{\text {fusion }}$ by a few $k_{B} T$. 


\subsubsection{Determination of vesicle release energy}

The distinction between the willing and reluctant vesicles will be left out of the notation in the following equations, although the processes described happen at both vesicle populations (see Appendix A for a complete description).

Contributions to $E_{\mathrm{SNARE}}$ come from the SNARE complexes associated with a vesicle, the number of which, $N_{\mathrm{SNARE}}$, has been determined to be at least three [Hua and Scheller, 2001; Domanska et al, 2009; Mohrmann et al, 2010]. In the DSCI model, $N_{\text {SNARE }}$ is fixed to a value of four per vesicle, with no variation in number between vesicle pools. The energetic contribution to $E_{\mathrm{SNARE}}$ also depends on the isoform of synaptotagmin bound to the SNARE complex [Zhang et al, 2010]. The presence of multiple isoforms of synaptotagmin suggests the possibility of an interaction between calcium sensors [Bhalla et al, 2005]. The DSCI model interprets this interaction as a competion for binding sites on the SNARE complexes.

Synaptotagmin 1 (syt1) is the primary calcium sensor in the rat CA1 [Berton et al, 1997], and has been well established to play the majority role in calcium-dependent vesicle exocytosis [Geppert et al, 1994; Yoshihara and Littleton, 2002]. Although the identity of a secondary calcium sensor has not yet been definitively determined, the existence of a secondary sensor has been established [Geppert et al, 1994; Kochubey and Schneggenburger, 2011]. The secondary sensor has been found to contribute to paired-pulse facilitation (and PPF was enhanced in syt1-null mutants, perhaps due to a reduction in the RRP) [Saraswati et al, 2007]. Another isoform of synaptotagmin is a natural candidate for the secondary sensor. 
After synaptotagmin isoforms 1 and 2, isoform 7 (syt7) is the most abundant brainspecific synaptotagmin isoform [Sugita et al, 2001]. Syt7 is expressed in the rat hippocampus [ibid], but whereas syt 1 and syt 2 are localized to the vesicle membrane, syt 7 is expressed in the plasma membrane, particularly at the active zone. Syt7 has been found to be involved in asynchronous release in CNS neurons [Wen et al, 2010 (although, see Saraswati et al, 2007; Maximov et al, 2008)]. In chromaffin cells, syt7 has been positively identified as the secondary calcium sensor [Schonn et al, 2008].

Synaptotagmin isoforms 2, 3, and 4 are also expressed in adult rat hippocampii, although not as strongly as syt1 [Berton et al, 1997]. Syt4 has been found to affect STP, particularly as a negative regulator of vesicle release [Ferguson et al, 2004], where it appears to be involved in fusion pore dilation [Zhang et al, 2010]. Syt7 has also been implicated in fusion pore stabilization and dilation [Segovia et al, 2010].

How would an asynchronous process contribute to the dynamics of synchronous release? One interpretation is that asynchronous release is a minor contributor to synchronous release, and that the asynchronous contribution increases during sustained activity as the willing pool is depleted [Maximov and Sudhof, 2005; Sun et al, 2007]. Indeed, specific knockdown of syt1 impairs, without completely abolishing, synchronous transmission, in what is observed to be a high-pass filtering of spike rate [Xu et al, 2012; Buzsaki, 2012]. In response to syt1 knockdown, single spikes fail to elicit responses, while high-frequency trains $(10 \mathrm{~Hz}$ to $50 \mathrm{~Hz})$, such as occur during bursts and gamma oscillations, alter the kinetics, but only slightly reduce the overall magni- 
tude of synaptic transmission. At very high frequencies (up to $200 \mathrm{~Hz}$ ), as occurs during sharp waves, the response is hardly impaired, except for a several millisecond delay (provided that there are at least 5 spikes in the stimulus). Xu et al attributed the altered behavior of transmission to an ancillary calcium sensor that is normally clamped in the presence of syt1.

At very low calcium concentrations, or essentially in the absence of calcium, the vesicle release machinery is clamped, preventing membrane fusion, while at higher calcium concentrations, vesicle release is promoted [Kochubey and Schneggenburger, 2011]. Synaptotagmin's actions have been explained either as inhibiting membrane fusion (i.e., acting as the clamp) [Popov and Poo, 1993], or as promoting membrane fusion [Walter et al, 2011]. However, synaptotagmin could in principle act as both clamp and promotor, depending on the calcium concentration [Kochubey et al, 2011].

The vesicle priming stage may also involve the displacement of complexin, another protein associated with the SNARE complex that plays a role in priming and clamping the RRP vesicles [Tang et al, 2006; Maximov et al, 2009; Yang et al, 2010]. The complexin clamp is not included in the DSCI model. However, the behavior of the less effective synaptotagmin isoform might be interpreted as an isoform-specific interaction with complexin, since deletions of either complexin or syt1 disinhibit the secondary calcium sensor [Yang et al, 2010]. In the DSCI model, the secondary calcium sensor is attributed to an isoform of synaptotagmin that competes more effectively for the synaptotagmin insertion site in the SNARE complex at lower calcium concentrations, yet 
delivers less energy to overcoming the membrane fusion barrier in high calcium concen. trations. The DSCI model also assumes that the SNARE complex is initially in the complexin-primed and clamped state.

In the DSCI model, the syt1 isoform does not saturate the SNARE binding sites due to differences in calcium-dependent kinetics (affinity and cooperativity) at the binding sites [Geppert, et al, 1994; Hui et al, 2005; Wen et al, 2010]. Syt7 has a greater binding affinity for calcium than does syt1 [Bhalla et al, 2005], so that at lower calcium concentrations, as occur in the residual cytosolic volume, syt7 outcompetes syt1 for available calcium, whereas during the rapid influx of calcium through VGCCs, syt1 can become fully calcium bound, and subsequently compete more effectively for positions on the SNARE complex. At synapses, however, syt1 and syt7 may not be "redundant", since syt 1 is a vesicle-bound protein, while syt7 is a plasma membrane-bound protein [Sugita et al, 2010]. Syt7 is not the only high-affinity syt isoform (others include 3, 5 and 10), so competition may exist between syt1 and another syt isoform.

In the absence of competition between syt isoforms, the fractional occupancy, $\Phi$, of the isoform sytX, at a SNARE complex (as a function of residual calcium concentration) is approximated by the Hill equation (assuming equilibrium kinetics, i.e., that the reactant concentrations change on a slower time scale than the processes of binding and unbinding - see Appendix B for a derivation of the Hill equation):

$$
\Phi_{\text {sytX,SNARE }}\left(\left[\mathrm{Ca}^{2+}\right]\right)=\frac{\left[\mathrm{Ca}^{2+}\right]^{n_{\text {Ca,sytX,SNARE }}}}{K_{\mathrm{Ca}, \text { SytX,SNANARE }}^{n_{\text {Ca,StXRE }}}+\left[\mathrm{Ca}^{2+}\right]^{n_{\text {Ca,sytX,SNARE }}}},
$$


where $n_{\text {Ca,sytX,SNARE }}$ is the calcium cooperativity of sytX-SNARE binding, and $K_{\mathrm{Ca}, \text {,ytXX,SNARE }}^{n_{\mathrm{C}, \mathrm{SN} \text {. }}}$ is the calcium concentration at half-maximal occupancy, or the microscopic dissociation constant (and since all binding sites are assumed identical, $K_{\text {Ca,sytX,SNARE }}$ is equal to the apparent dissociation constant). The affinity of calcium binding to syt1 is about $20 \mu \mathrm{M}$ [Sheng et al, 1996; Sugita et al, 2010], and the cooperativity is between 2 and 5 [Neher and Sakaba, 2008]. The kinetics of the sytX-SNARE binding process, assuming that any intermediate forms are very short-lived, can be written as:

$$
\mathrm{SytX}+n \mathrm{Ca}^{2+}+\mathrm{SNARE} \underset{k_{\text {off }}}{\stackrel{k_{\text {on }}}{\rightleftharpoons}} \mathrm{SytXCaSNARE}
$$

which is equivalent to:

$$
\frac{d}{d t} \operatorname{SytXCaSNARE}=k_{\mathrm{on}} \operatorname{SytX} \times \operatorname{SNARE}\left[\mathrm{Ca}^{2+}\right]^{n}-k_{\text {off }} \text { SytXCaSNARE. }
$$

Since $[\mathrm{SytX}]$ can be assumed constant, this simplifies to:

$$
\frac{d}{d t} \operatorname{SytXCaSNARE}=\hat{k}_{\text {on }} \operatorname{SNARE}\left[\mathrm{Ca}^{2+}\right]^{n}-k_{\text {off }} \text { SytXCaSNARE, }
$$

where $\hat{k}_{\text {on }}=k_{\text {on }}$ SytX. Since the number of SNARE complexes is constant, the SNARE concentration is equal to $N_{\mathrm{SNARE}}-\mathrm{SytXCaSNARE}$. This substitution yields:

$$
\begin{gathered}
\frac{d}{d t} \text { SytXCaSNARE }= \\
\hat{k}_{\text {on }}\left(N_{\text {SNARE }}-\text { SytXCaSNARE }\right)\left[\mathrm{Ca}^{2+}\right]^{n}-k_{\text {off }} \text { SytXCaSNARE, }
\end{gathered}
$$

or, after simplification, 


$$
\frac{d}{d t} \operatorname{SytXCaSNARE}=\hat{\hat{k}}_{\mathrm{on}}\left[\mathrm{Ca}^{2+}\right]^{n}-\left(\hat{\hat{k}}_{\mathrm{on}}\left[\mathrm{Ca}^{2+}\right]^{n}+k_{\text {off }}\right) \text { SytXCaSNARE, }
$$

where $\hat{\hat{k}}_{\text {on }}=\hat{k}_{\text {on }} N_{\text {SNARE }}$. The fractional occupancy of SytX on a SNARE complex, $\Phi_{\text {sytX,SNARE }}$, is converted to a probability of occupancy through the linear transformation:

$$
\begin{gathered}
p_{\text {sytX,SNARE }}\left(\left[\mathrm{Ca}^{2+}\right]\right)= \\
p_{\text {sytX,SNARE,min }}+\left(p_{\text {sytX,SNARE,max }}-p_{\text {sytX,SNARE,min }}\right) \Phi_{\text {sytX,SNARE }}\left(\left[\mathrm{Ca}^{2+}\right]\right),
\end{gathered}
$$

where $p_{\text {sytX,SNARE,max }}$ and $p_{\text {sytX,SNARE,min }}$ are the maximum and minimum probabilities of sytX occupancy at SNARE sites as a function of residual calcium concentration. A differential form of $p_{\text {sytX,SNARE }}$ can be found by substitution:

$$
\begin{aligned}
& p_{\text {sytX,SNARE }}= \\
& p_{\text {sytX,SNARE,min }}+\left(p_{\text {sytX,SNARE,max }}-p_{\text {sytX,SNARE,min }}\right) \frac{\text { SytXCaSNARE }}{N_{\text {SNARE }}} \\
& \frac{d}{d t} p_{\text {SytX,SNARE }}=\frac{\left(p_{\text {sytX,SNARE,max }}-p_{\text {sytX,SNARE,min }}\right)}{N_{\text {SNARE }}} \frac{d}{d t} \text { SytXCaSNARE } \\
& \frac{d}{d t} p_{\mathrm{SytX}, \mathrm{SNARE}}=\frac{\left(p_{\mathrm{SytX}, \mathrm{SNARE}, \mathrm{max}}-p_{\mathrm{SytX}, \mathrm{SNARE}, \mathrm{min}}\right)}{N_{\mathrm{SNARE}}}[ \\
& \left.\hat{\hat{k}}_{\text {on }}\left[\mathrm{Ca}^{2+}\right]_{\text {res }}^{n_{\text {Ca,syX }, \text { SNARE }}}-\left(\hat{\hat{k}}_{\text {on }}\left[\mathrm{Ca}^{2+}\right]_{\text {res }}^{n_{\text {Ca,syt } X \text { SNARE }}}+k_{\text {off }}\right) \text { SytXCaSNARE }\right] .
\end{aligned}
$$

The calcium-dependent probability that competition will occur at a binding site, given the individual calcium-dependent probabilities of syt occupancy, is calculated as the probability of the events occurring simultaneously:

$$
p_{\text {comp }}\left(\left[\mathrm{Ca}^{2+}\right]\right)=p_{\text {syt } 1, \mathrm{SNARE}}\left(\left[\mathrm{Ca}^{2+}\right]\right) p_{\text {syt7, SNARE }}\left(\left[\mathrm{Ca}^{2+}\right]\right) .
$$


Since willing and reluctant vesicles are treated independently, probabilities of competition have to be considered for both vesicle types. To get the binding probabilities of each syt isoform (which can add to values less than one), the probability of competition is subtracted from the probability of occupancy for each syt isoform. The probability that each isoform will win a competition is then added back in, resulting in:

$$
\hat{p}_{\text {sytX,SNARE }}=p_{\text {sytX,SNARE }}-p_{\text {comp }}+p_{\text {sytX,comp }},
$$

where the $\left[\mathrm{Ca}^{2+}\right]$ notation has been dropped. The probability that syt 1 will win a compe. tition is given by:

$$
p_{\mathrm{syt} 1, \mathrm{comp}}=\frac{p_{\mathrm{comp}}}{\frac{p_{\mathrm{syt}, \mathrm{SNARE}}}{p_{\mathrm{syt} 1, \mathrm{SNARE}}}+1},
$$

and similarly for $p_{\text {syt7,comp }}$. The number of syt isoforms at each vesicle type is given by:

$$
N_{\text {sytX }}=\hat{p}_{\text {sytX,SNARE }} N_{\text {SNARE }} \text {. }
$$

To calculate $E_{\mathrm{SNARE}}$, the calcium-dependent kinetics of SNARE-mediated vesicle fusion for each syt isoform must be considered. The concentration of nanodomain calcium is different for the two vesicle types: for willing vesicles, the calcium concentra tion during a spike is the full nanodomain concentration, whereas for the reluctant vesicles, the calcium concentration during a spike is approximated by a fixed fraction, $\phi_{\mathrm{Ca}, \mu}$, of the full nanodomain concentration. Given the nanodomain calcium concentration, the fraction of syt-bound SNARE complexes that trigger membrane fusion as a function of calcium (for each syt isoform), $\Phi_{\text {sytX,willing }}\left(\left[\mathrm{Ca}^{2+}\right]_{\mu}\right)$, can be approximated by the Hill equation (again assuming equilibrium kinetics): 


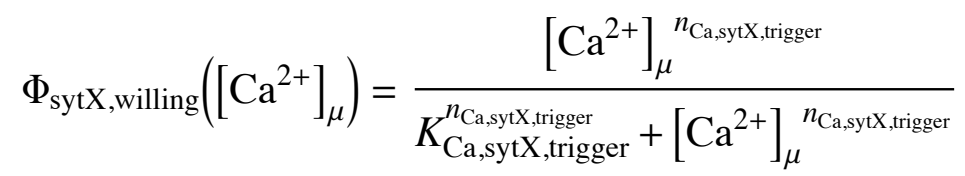

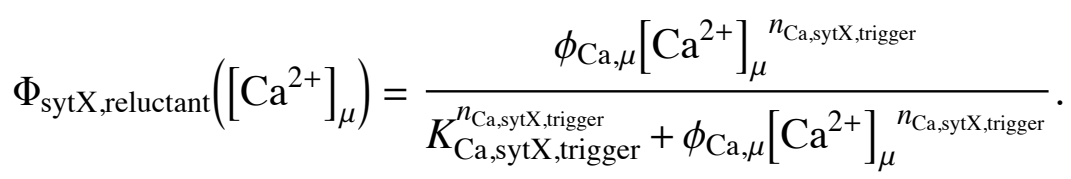

The assumption of equilibrium kinetics may be more questionable during this process, though, since some intermediate states of the SNARE complex have rate constants in the msec range [Pan and Zucker, 2009].

The fractional conformational change can then be multiplied by $N_{\text {sytX }}$ for each vesicle type, converting it to a number:

$$
\hat{N}_{\text {sytX }}\left(\left[\mathrm{Ca}^{2+}\right]_{\mu}\right)=N_{\text {sytX }} \Phi_{\text {sytX }}\left(\left[\mathrm{Ca}^{2+}\right]_{\mu}\right) .
$$

Finally, the energetic contribution to membrane fusion for each vesicle type can be determined. For the willing vesicles,

$$
E_{\text {SNARE }}\left(\left[\mathrm{Ca}^{2+}\right]_{\mu}\right)=E_{\text {syt } 1} \hat{N}_{\text {syt } 1}\left(\left[\mathrm{Ca}^{2+}\right]_{\mu}\right)+E_{\text {syt } 7} \hat{N}_{\text {syt } 7}\left(\left[\mathrm{Ca}^{2+}\right]_{\mu}\right),
$$

and similarly for the reluctant vesicles.

\subsubsection{Probability of release}

Vesicle release is assumed to be uniquantal. The probability of quantal release is conveniently written in terms of the probability of the failure of a vesicle to exocytose: $1-P_{\text {ves }}$. For $n$ vesicles in the primed state, the probability that none of the vesicles release a quantum of neurotransmitter is $\left(1-P_{\text {ves }}\right)^{n}$. Thus, the probability that at least one vesicle will release is $1-\left(1-P_{\mathrm{ves}}\right)^{n}$. Given two types of vesicles, the overall 
release probability is one minus the probability of both types failing to release:

$$
P_{\text {release }}=1-\left(1-P_{\text {ves, willing }}\right)^{n_{\text {ves, willing }}}\left(1-P_{\text {ves, reluctant }}\right)^{n_{\text {ves, reluctant }}} .
$$

Several pieces of experimental evidence suggest this release probability at excitatory CA1 synapses is on average between about 0.2 and 0.4 at rest [Hjelmstad et al, 1997].

\subsection{Behavior of the DSCI model}

The behavior of the DSCI model of STP is documented in this section. First, values for the model parameters are reported. Then the release probability behavior of the model is compared to the Frerking data. The residual calcium, the vesicle pool populations, and the occupancy of syt isoforms on the SNARE complexes, are also shown.

Deviations from the data are also examined. First, the errors are shown vs. stimuli. Then the response of the errors to perturbations in each parameter are shown (also vs. stimuli).

\subsubsection{Parameter optimization}

The model parameters, their estimated bounds, and sources for the estimations, are shown in Table 2.3. The bounds in the parameter estimations are due to measurement uncertainties and differences between sample preparations and experimental techniques. The influence of a change in any given parameter is difficult to predict due to the nonlinear interactions with other parameters. However, the problem of finding exact parameter values can be treated as a constrained optimization problem. Appendix C discusses the optimization methods that were applied to the problem of determining 
exact parameter values.

The results of parameter optimization are listed in Table 2.4. Data set \#1 is the original Frerking data shown in Figs. 2.1 and 2.2. Data set \#2 is another set of data collected by Matt Frerking in March, 2007. The same set of ISIs was used in the collection of data set \#2. The reponse characteristics of data set \#2 are different than data set \#1. Most notably, the high frequency response in data set \#2 shows a larger facilitation with a corresponding lower depression. Also, the large depressive deflections seen in the complex trains in data set \#1 are absent in data set \#2. The parameters fit to data set \#1 do not describe data set \#2. However, by allowing only the calcium and vesicle refillling parameters to vary, a reasonable fit to data set \#2 was obtained, indicating the generalizability of the model. Table 2.4 summarizes the optimized parameters for both data sets. In both tables, red shading indicates fixed parameters. In Table 2.4, orange shading indicates parameters that were fixed after fitting to data set \#1, and yellow shading indicates parameters that reached a bound during fitting. 
Table 2.3. The DSCI model parameter ranges (see text).

\begin{tabular}{|c|c|c|c|c|}
\hline Parameter & Min & $\operatorname{Max}$ & Units & Source \\
\hline$\left[\mathrm{Ca}^{2+}\right]_{\mathrm{res}, 0}$ & 0.0316228 & 0.1 & $\mu \mathrm{M}$ & $\begin{array}{c}\text { [Sinha et al,1997; Yao et } \\
\text { al,2009;Nadkarni et al,2010] }\end{array}$ \\
\hline$\Delta_{\mathrm{Ca}, \mu}$ & 30 & 160 & $\mu \mathrm{M}$ & [Augustine et al,2003] \\
\hline$\Delta_{\mathrm{Ca}, \text { int }}$ & 0.05 & 1.25 & $\mu \mathrm{M}$ & [Helmchen et al,1997;Muller et al,2007] \\
\hline$\Delta_{\text {Ca,slow }}$ & 0.005 & 0.015 & $\mu \mathrm{M}$ & [Helmchen et al,1997;Muller et al,2007] \\
\hline$\overline{\tau_{\mathrm{Ca}, \text { int }}}$ & 0.025 & 0.1 & $\mathrm{~s}$ & [Helmchen et al,1997;Muller et al,2007] \\
\hline$\tau_{\text {Ca,slow }}$ & 1 & 10 & $\mathrm{~s}$ & [Helmchen et al,1997;Muller et al,2007] \\
\hline$n_{\text {rest }}$ & 2 & 10 & $\mathbb{R}$ & [Harris and Sultan,1995] \\
\hline$\phi_{n}$ & 0.1 & 0.9 & fraction & [Lee (JS) et al,2012] \\
\hline$\tau_{\text {fast }}$ & 0.45 & 5 & $\mathrm{~s}$ & [Otsu et al,2004] \\
\hline$\tau_{\text {slow }}$ & 5 & 25 & $\mathrm{~s}$ & [Otsu et al,2004] \\
\hline$\tau_{k, \text { refill }}$ & 1 & 20 & $\mathrm{~s}$ & {[]} \\
\hline $\mathrm{Amp}_{\text {refill,fast }}$ & 0.1 & 15 & $\mathbb{R}$ & [] \\
\hline $\mathrm{Kd}_{\text {refill,fast }}$ & 0.01 & 1 & $\mu \mathrm{M}$ & [] \\
\hline$\phi_{\mathrm{Ca}, \mu}$ & 0.05 & 1 & fraction & {[]} \\
\hline$p_{\text {syt } 1, \text { SNARE,min,willing }}$ & 0 & 1 & probability & {[]} \\
\hline$p_{\text {syt1,SNARE,min,reluctant }}$ & 0 & 1 & probability & {[]} \\
\hline$p_{\text {syt7,SNARE,min }}$ & 0.5 & 1 & probability & {[]} \\
\hline$p_{\text {syt1,SNARE,max,willing }}$ & 1 & 1 & probability & {[]} \\
\hline$p_{\text {syt1,SNARE,max,reluctant }}$ & 1 & 1 & probability & {[]} \\
\hline$p_{\text {syt7,SNARE,max }}$ & 1 & 1 & probability & {[]} \\
\hline$K_{\text {Ca,syt } 1, \text { SNARE }}$ & 0.01 & 0.5 & $\mu \mathrm{M}$ & [Bhalla et al,2005;Sun et al,2007] \\
\hline$K_{\mathrm{Ca}, \text { syt7,SNARE }}$ & 0.005 & 0.2 & $\mu \mathrm{M}$ & [Bhalla et al,2005;Sun et al,2007] \\
\hline$K_{\mathrm{Ca}, \text { syt1, trigger }}$ & 20 & 20 & $\mu \mathrm{M}$ & [Sun et al,2007] \\
\hline$K_{\mathrm{Ca}, \text { syt7, trigger }}$ & 0 & 5 & $\mu \mathrm{M}$ & [Sun et al,2007] \\
\hline$\overline{E_{\mathrm{syt} 1}}$ & 9 & 15 & $\mathrm{k}_{\mathrm{B}} \mathrm{T}$ & [] \\
\hline$E_{\text {syt7 }}$ & 7 & 15 & $\mathrm{k}_{\mathrm{B}} \mathrm{T}$ & [] \\
\hline$E_{\text {fusion }}$ & 40 & 40 & $\mathrm{k}_{\mathrm{B}} \mathrm{T}$ & [Li et al,2007] \\
\hline$N_{\text {SNARE }}$ & 4 & 4 & $\mathbb{Z}$ & $\begin{array}{c}\text { [Hua and Scheller,2001; } \\
\text { Mohrmann et al,2010] }\end{array}$ \\
\hline$n_{\text {Ca,syt1,trigger }}$ & 2 & 4.5 & $\mathbb{R}$ & [Sun et al,2007] \\
\hline$n_{\mathrm{Ca}, \text { syt7,trigger }}$ & 0.5 & 4 & $\mathbb{R}$ & [Sun et al,2007] \\
\hline$n_{\text {Ca,syt } 1, \text { SNARE }}$ & 1 & 3 & $\mathbb{R}$ & [Sun et al,2007] \\
\hline$n_{\text {Ca,syt7,SNARE }}$ & 1 & 3 & $\mathbb{R}$ & [Sun et al,2007] \\
\hline
\end{tabular}


Table 2.4. Optimized parameters for the DSCI model (see text).

\begin{tabular}{|c|c|c|c|c|c|}
\hline Parameter & Data Set 1 & Data Set 2 & Min & Max & Units \\
\hline$\left[\mathrm{Ca}^{2+}\right]_{\mathrm{res}, 0}$ & 0.0960727 & 0.1 & 0.0316228 & 0.1 & $\mu \mathrm{M}$ \\
\hline$\Delta_{\mathrm{Ca}, \mu}$ & 124.102 & 105.061 & 30 & 160 & $\mu \mathrm{M}$ \\
\hline$\Delta_{\mathrm{Ca} \text {,int }}$ & 1.1043 & 1.0692 & 0.05 & 1.25 & $\mu \mathrm{M}$ \\
\hline$\Delta_{\text {Ca,slow }}$ & 0.0057 & 0.015 & 0.005 & 0.015 & $\mu \mathrm{M}$ \\
\hline$\tau_{\mathrm{Ca}, \text { int }}$ & 0.025 & 0.025 & 0.025 & 0.1 & $\mathrm{~S}$ \\
\hline$\tau_{\text {Ca,slow }}$ & 5.7719 & 3.7538 & 1 & 10 & $\mathrm{~S}$ \\
\hline$n_{\text {rest }}$ & 4.7765 & 2 & 2 & 10 & $\mathbb{R}$ \\
\hline$\phi_{n}$ & 0.7314 & 0.7323 & 0.1 & 0.9 & fraction \\
\hline$\tau_{\text {fast }}$ & 0.45 & 0.45 & 0.45 & 5 & $\mathrm{~S}$ \\
\hline$\tau_{\text {slow }}$ & 25 & 25 & 5 & 25 & $\mathrm{~s}$ \\
\hline$\tau_{k, \text { refill }}$ & 4.6449 & 3.9099 & 1 & 20 & $\mathrm{~s}$ \\
\hline $\mathrm{Amp}_{\text {refill,fast }}$ & 15 & 15 & 0.1 & 15 & $\mathbb{R}$ \\
\hline $\mathrm{Kd}_{\text {refill,fast }}$ & 0.0089 & 0.01 & 0.01 & 1 & $\mu \mathrm{M}$ \\
\hline$\phi_{\mathrm{Ca}, \mu}$ & 1 & 1 & 0.05 & 1 & fraction \\
\hline$p_{\text {syt1,SNARE,min,willing }}$ & 0.8075 & 0.8075 & 0 & 1 & probability \\
\hline$p_{\text {syt1,SNARE,min,reluctant }}$ & 0.8075 & 0.8075 & 0 & 1 & probability \\
\hline$p_{\text {syt } 7, \text { SNARE,min }}$ & 0.8075 & 0.8075 & 0.5 & 1 & probability \\
\hline$p_{\text {syt } 1, \text { SNARE,max,willing }}$ & 1 & 1 & 1 & 1 & probability \\
\hline$p_{\text {syt } 1, \text { SNARE,max,reluctant }}$ & 1 & 1 & 1 & 1 & probability \\
\hline$p_{\text {syt7,SNARE, } \max }$ & 1 & 1 & 1 & 1 & probability \\
\hline$K_{\mathrm{Ca} \text {,syt } 1 \text {,SNARE }}$ & 0.0336 & 0.0556 & 0.01 & 0.5 & $\mu \mathrm{M}$ \\
\hline$K_{\mathrm{Ca} \text {,syt7,SNARE }}$ & 0.0336 & 0.0556 & 0.005 & 0.2 & $\mu \mathrm{M}$ \\
\hline$K_{\mathrm{Ca} \text {,syt1,trigger }}$ & 20 & 20 & 20 & 20 & $\mu \mathrm{M}$ \\
\hline$K_{\mathrm{Ca} \text {,syt7,trigger }}$ & 3.7803 & 3.7803 & 0 & 5 & $\mu \mathrm{M}$ \\
\hline$E_{\text {syt1 }}$ & 11.49 & 11.49 & 9 & 15 & $\mathrm{k}_{\mathrm{B}} \mathrm{T}$ \\
\hline$E_{\text {syt7 }}$ & 8.2249 & 8.2249 & 7 & 15 & $\mathrm{k}_{\mathrm{B}} \mathrm{T}$ \\
\hline$E_{\text {fusion }}$ & 40 & 40 & 40 & 40 & $\mathrm{k}_{\mathrm{B}} \mathrm{T}$ \\
\hline$N_{\text {SNARE }}$ & 4 & 4 & 4 & 4 & $\mathbb{Z}$ \\
\hline$n_{\mathrm{Ca} \text {,syt } 1 \text {,trigger }}$ & 2.1887 & 2.1887 & 2 & 4.5 & $\mathbb{R}$ \\
\hline$n_{\mathrm{Ca}, \text { syt } 7 \text {,trigger }}$ & 0.6882 & 0.6882 & 0.5 & 4 & $\mathbb{R}$ \\
\hline$n_{\mathrm{Ca}, \mathrm{syt} 1, \mathrm{SNARE}}$ & 1.5609 & 1.5609 & 1 & 3 & $\mathbb{R}$ \\
\hline$n_{\mathrm{Ca}, \text { syt7,SNARE }}$ & 2.5654 & 2.5654 & 1 & 3 & $\mathbb{R}$ \\
\hline
\end{tabular}




\subsubsection{The DSCI model explains the Frerking data}

The fits of the DSCI model to the Frerking data, corresponding to the parameters listed in Table 2.4, are shown in Figs. 2.8-2.11. For each data set, fits to the training and testing data are shown. Goodness of fit is indicated by the squared correlation coefficient, or coefficient of determination, which quantifies the explanation of variance. The total combined-data coefficient of determination of the DSCI model for data set \#1 is $r^{2}=0.81$. The total combined-data coefficient of determination of the DSCI model for data set $\# 2$ is $r^{2}=0.34$. Scatter plots of both data sets are shown in Fig. 2.7. One cause of the lower $r^{2}$ for data set $\# 2$ is an anomolous signal in the $2 \mathrm{~Hz}$ data (Fig. 2.9E). Without the $2 \mathrm{~Hz}$ ordered data, the $r^{2}$ for data set $\# 2$ is 0.45 .
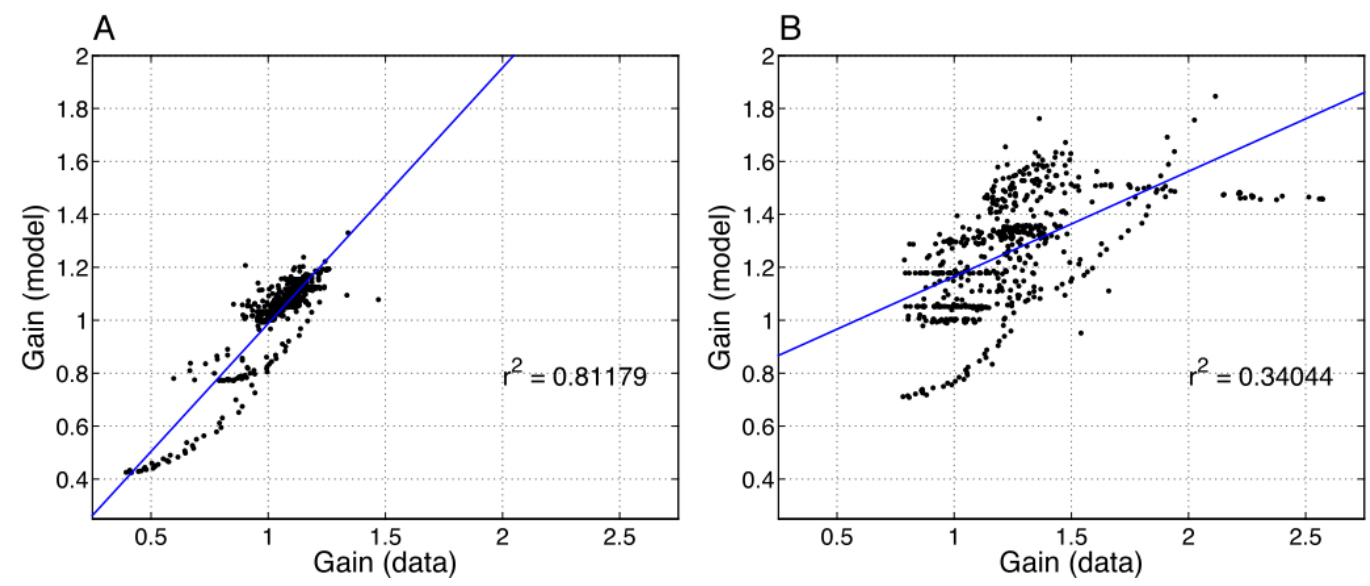

Figure 2.7. DSCI model correlations to all data. (A) data set \#1. (B) data set \#2. 
A

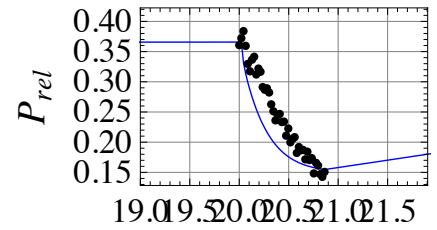

$\mathrm{C}$

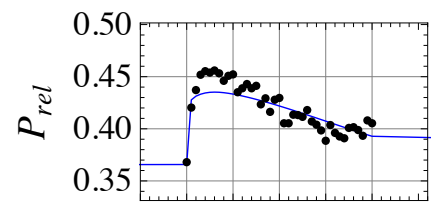

19202122232425

E

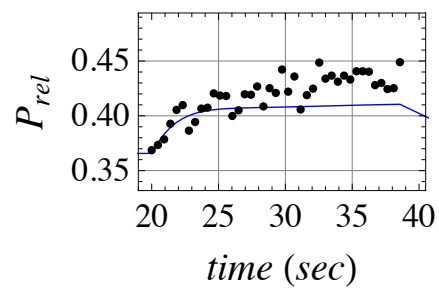

B

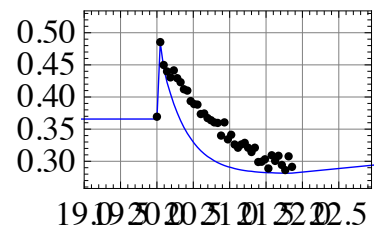

D

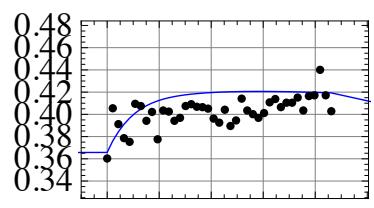

$\begin{array}{llllll}20 & 22 & 24 & 26 & 28 & 30\end{array}$

$\mathrm{F}$

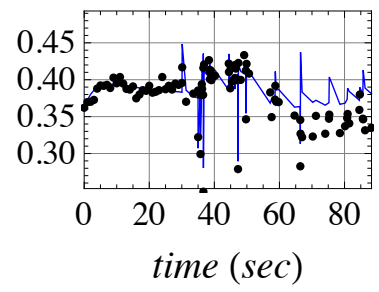

Figure 2.8. DSCI model fit to training data from data set \#1. Blue line is model, black points are data. A) $50 \mathrm{~Hz}$, B) $20 \mathrm{~Hz}$, C) $10 \mathrm{~Hz}$, D) $5 \mathrm{~Hz}$, E) $2 \mathrm{~Hz}$, F) complex train \#1.

A

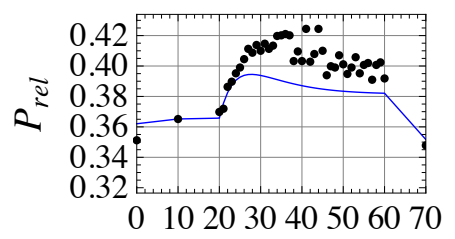

$\mathrm{C}$

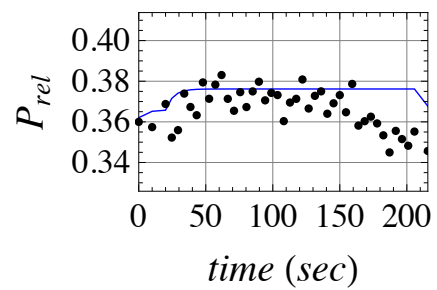

B

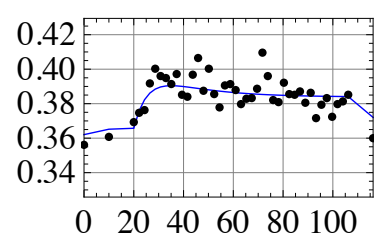

D

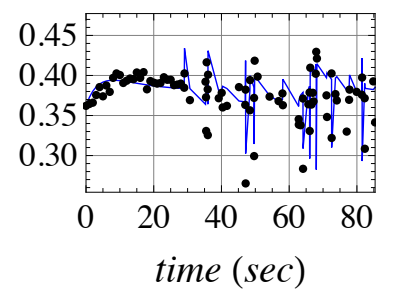

Figure 2.9. DSCI model fit to testing data from data set \#1. Blue line is model, black points are data. A) $1 \mathrm{~Hz}, \mathrm{~B}) 0.5 \mathrm{~Hz}, \mathrm{C}) 0.2 \mathrm{~Hz}, \mathrm{D})$ complex train \#2. 
A

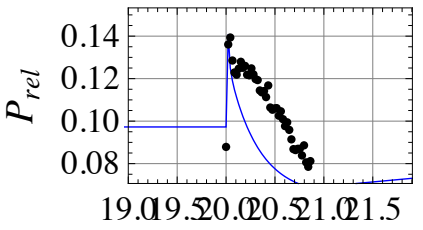

$\mathrm{C}$

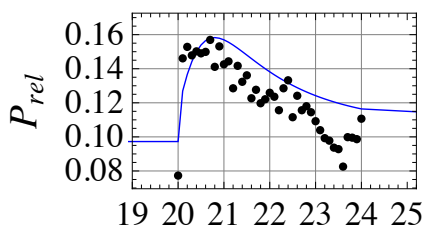

$\mathrm{E}$

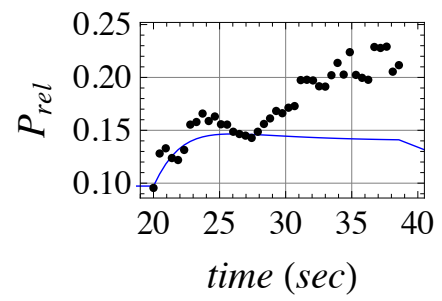

B

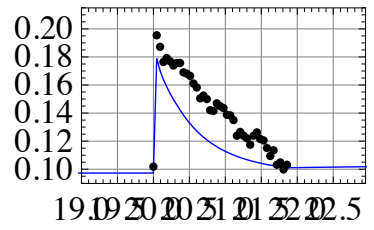

$\mathrm{D}$

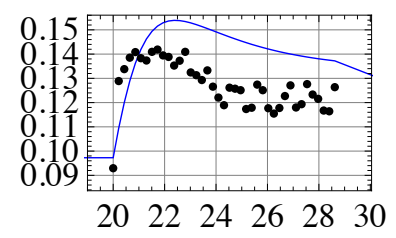

$\mathrm{F}$

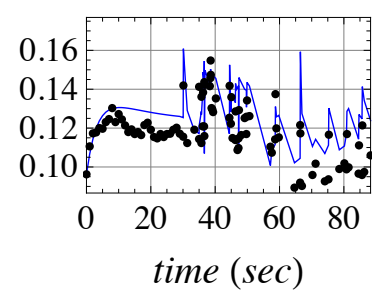

Figure 2.10. DSCl model fit to training data from data set \#2. Blue line is model, black points are data. A) $50 \mathrm{~Hz}$, B) $20 \mathrm{~Hz}$, C) $10 \mathrm{~Hz}$, D) $5 \mathrm{~Hz}$, E) $2 \mathrm{~Hz}$, F) complex train $\# 1$.

A

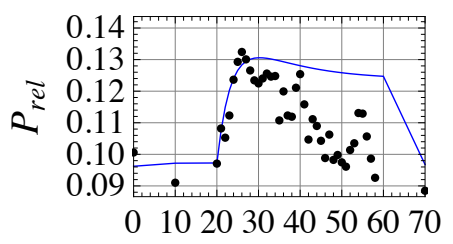

$\mathrm{C}$

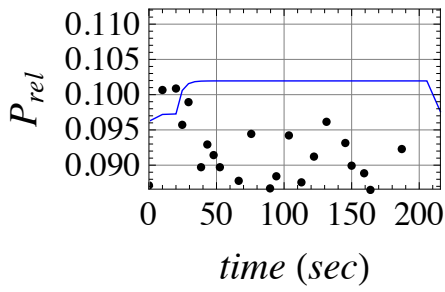

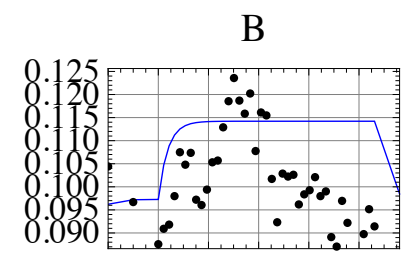

$\begin{array}{llllll}0 & 20 & 40 & 60 & 80 & 100\end{array}$

D

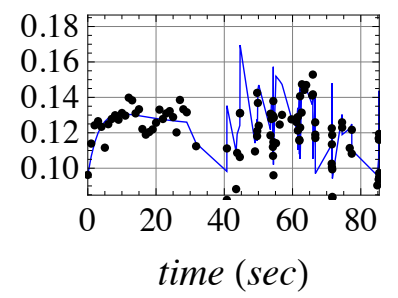

Figure 2.11. DSCI model fit to testing data from data set \#2. Blue line is model, black points are data. A) $1 \mathrm{~Hz}, B) 0.5 \mathrm{~Hz}, \mathrm{C}) 0.2 \mathrm{~Hz}, \mathrm{D})$ complex train \#2. 


\subsubsection{Calcium behavior in the DSCI model}

Residual calcium is recorded at the stimulation times, and builds to the expected level of $\sim 1 \mu \mathrm{M}$ during high-frequency stimulation (Fig. 2.12) [Tank et al, 1995; Helmchen et al, 1997].

A
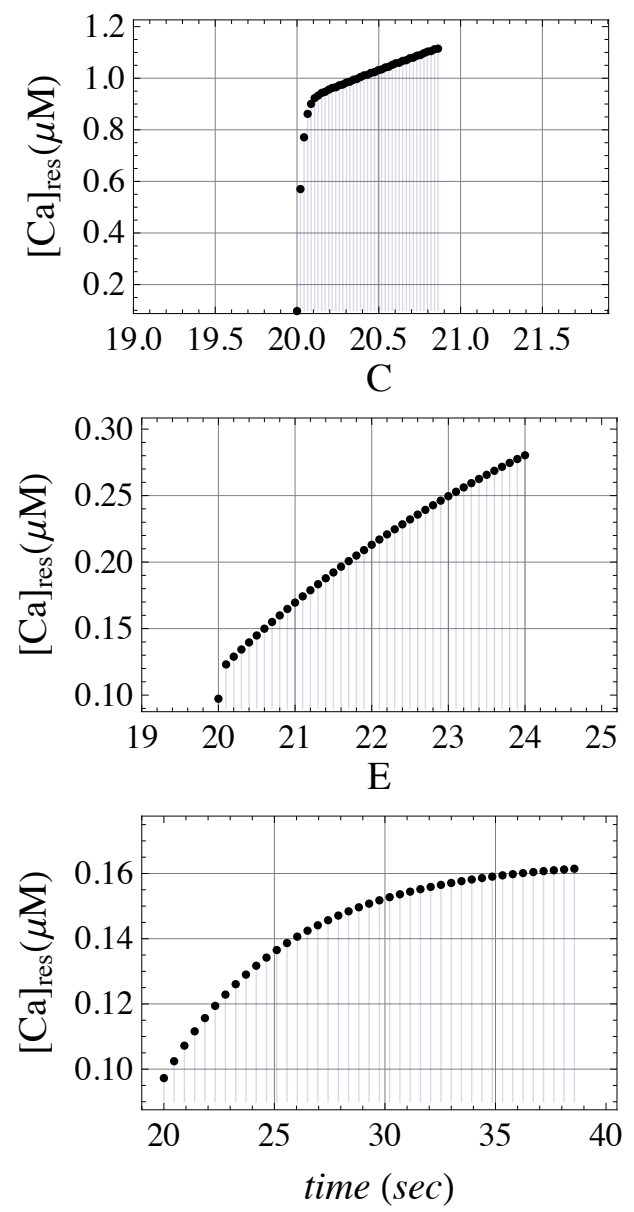

B

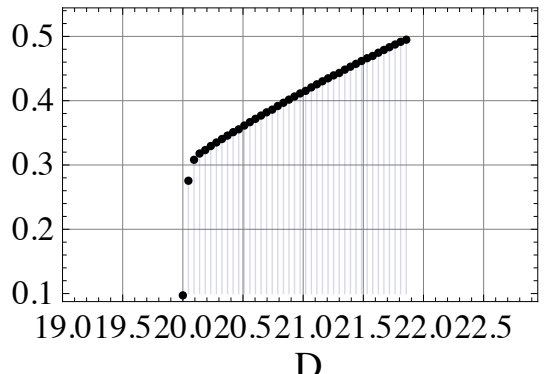

$\mathrm{D}$
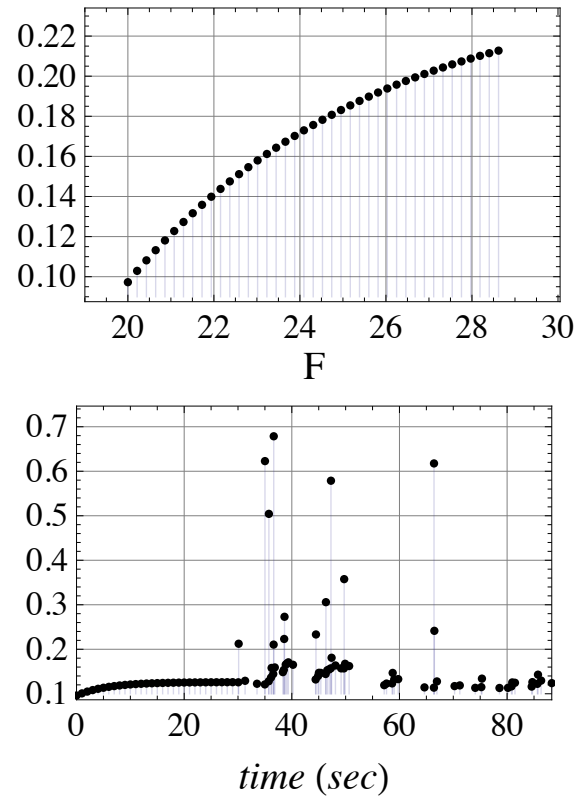

Figure 2.12. Residual calcium at spike times in data set $\# 1$. A) $50 \mathrm{~Hz}, B) 20 \mathrm{~Hz}$, C) $10 \mathrm{~Hz}$, D) $5 \mathrm{~Hz}$, E) $2 \mathrm{~Hz}, \mathrm{~F}$ ) complex train \#1. 


\section{Calcium dependency of release probability}

In receptor-ligand binding kinetics, the cooperativity of the reaction is approximated by the slope of the log-log relationship of the ligand concentration to the ratio of the fraction, $\theta /(1-\theta)$, of occupied to unoccupied binding sites. This relation is attained by a reformulation of the Hill equation (also see Appendix B):

$$
\begin{gathered}
\theta=\frac{[L]^{n}}{K_{d}+[L]^{n}} \\
\frac{\theta}{1-\theta}=\frac{[L]^{n}}{K_{d}} \\
\log \left(\frac{\theta}{1-\theta}\right)=n \log ([L])-\log \left(K_{d}\right)
\end{gathered}
$$

In the approximation of synaptic vesicle release to a receptor-ligand binding reaction, the release rate, or release probability, is equated with the left-hand side of Eqn. (2.59), and the slope, $n$, is interpreted to be the number of $\mathrm{Ca}^{2+}$ ions that cooperatively bind to each SNARE complex in the release mechanism. Although the relationship seen in Fig. 2.13 is not linear, the log-log relationship of calcium to release rate has been observed to similarly bend over at high calcium concentration [Sun et al, 2007]. 


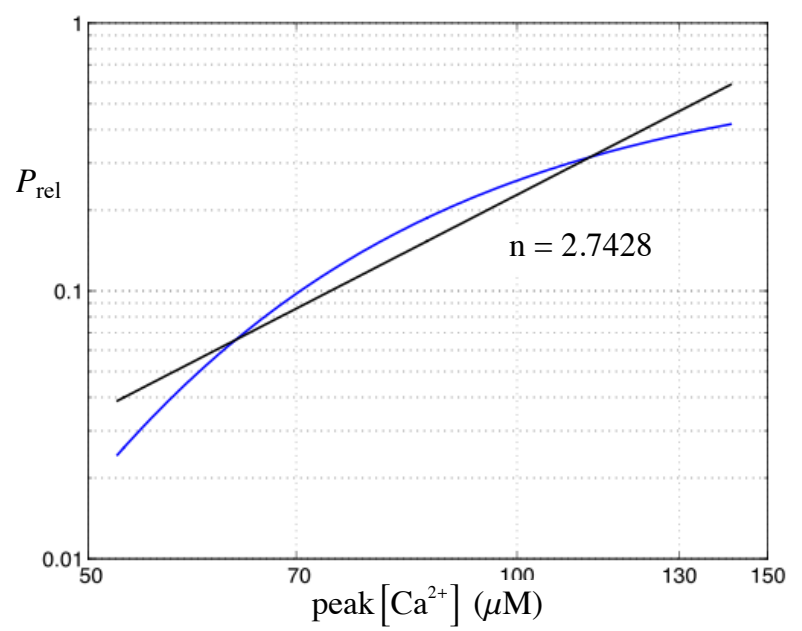

Figure 2.13. Calcium dependency of vesicle release probability in the $\mathrm{DSCl}$ model. Mean slope is interpreted to be an approximation of the cooperativity of peak release.

\subsubsection{Vesicle pool behavior}

The kinetics of the willing and reluctant vesicle pools are shown in Fig. 2.14. Because the two vesicle pools experience the same calcium signal, the ratio of syt 1 to syt7 is the same for both vesicle pools, and therefore the individual vesicle release probabilities are the same for the two pools. The only difference in contribution to total release probability from the two pools is the number of vesicles (Eqn. 2.56). 
A
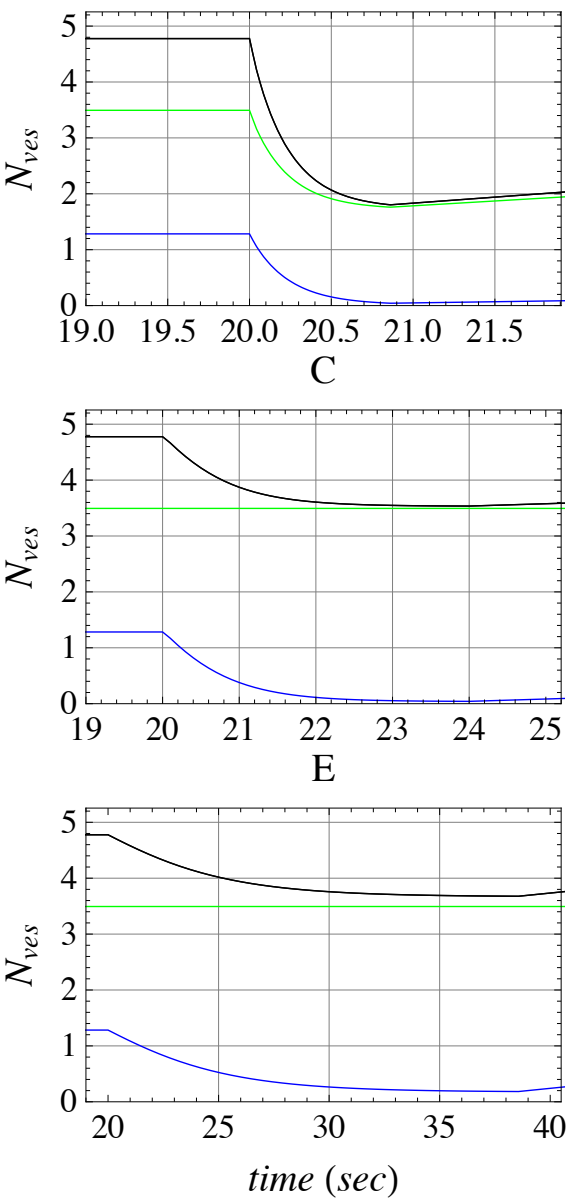

$\mathrm{B}$
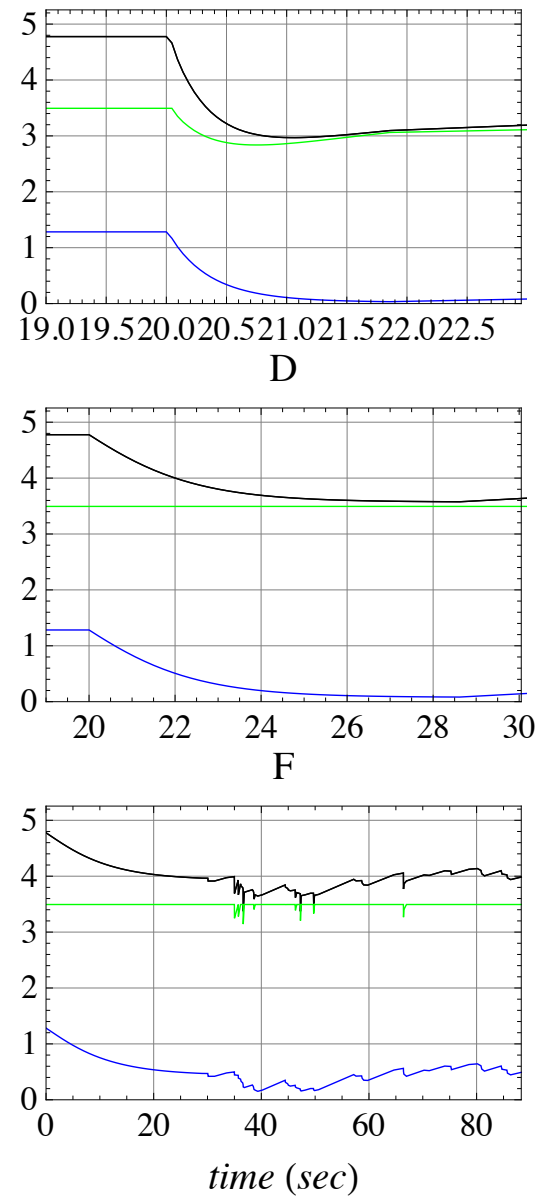

Figure 2.14. Vesicle pool populations at spike time in data set \#1. Green line: willing vesicles. Blue line: reluctant vesicles. Black line: all vesicles. A) $50 \mathrm{~Hz}, \mathrm{~B}) 20 \mathrm{~Hz}, \mathrm{C}$ ) $10 \mathrm{~Hz}$, D) $5 \mathrm{~Hz}$, E) $2 \mathrm{~Hz}, \mathrm{~F}$ ) complex train \#1. 


\subsubsection{Synaptotagmin occupancies}

The ISI-dependency of synaptotagmin isoform fraction on SNARE complexes is shown in Fig. 2.15.

A
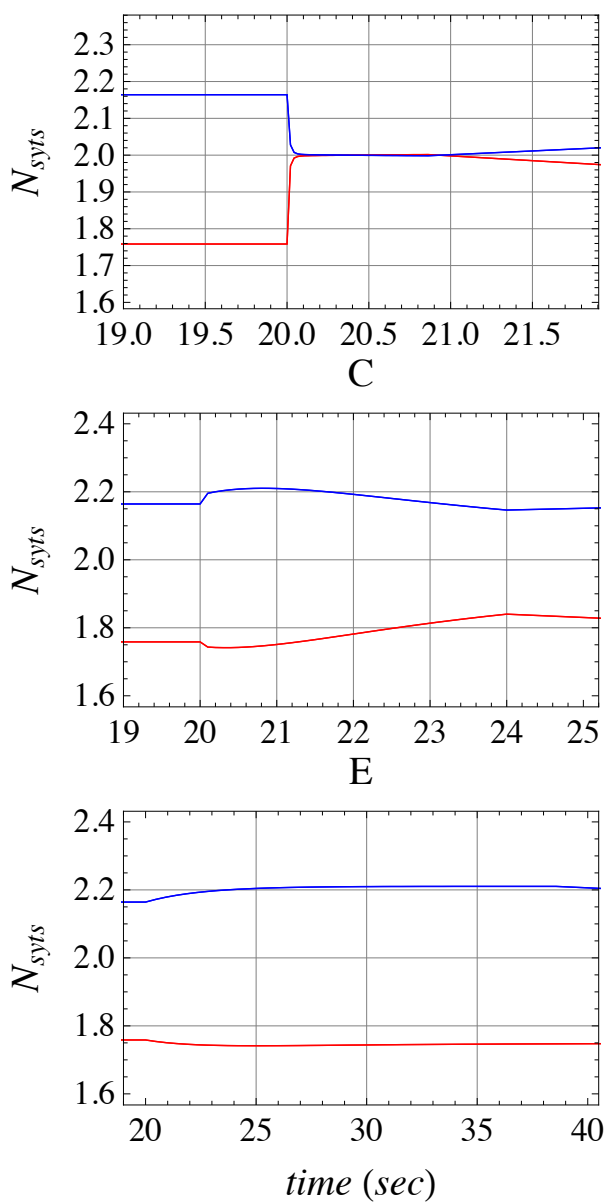

B
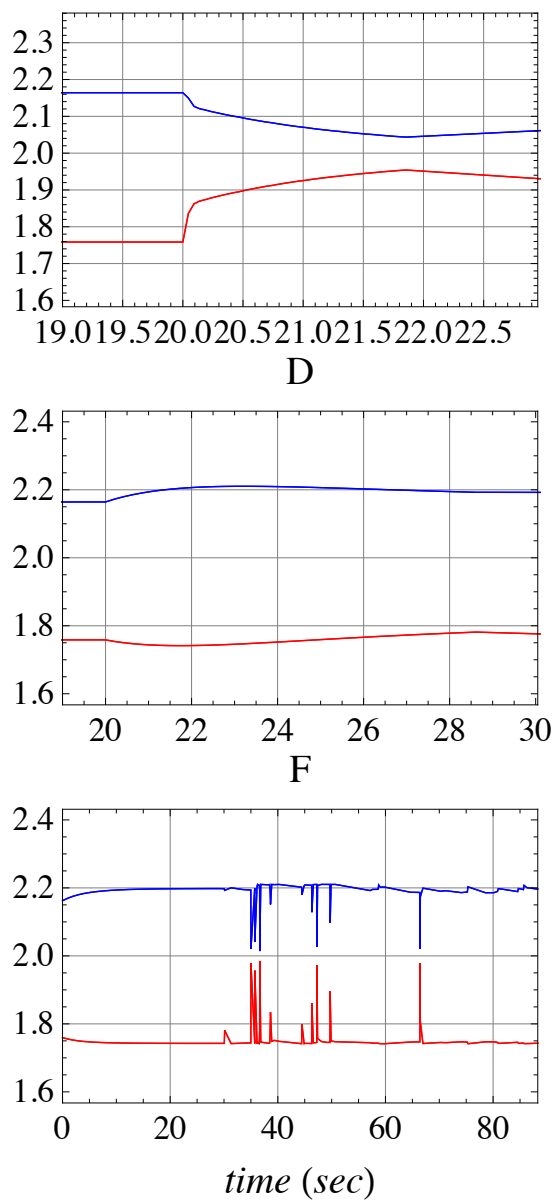

Figure 2.15. Synaptotagmin isoform complements at spike times in data set \#1. Red line: Syt7. Blue line: Syt1. A) $50 \mathrm{~Hz}$, B) $20 \mathrm{~Hz}$, C) $10 \mathrm{~Hz}$, D) $5 \mathrm{~Hz}$, E) $2 \mathrm{~Hz}$, F) complex train \#1. 
A
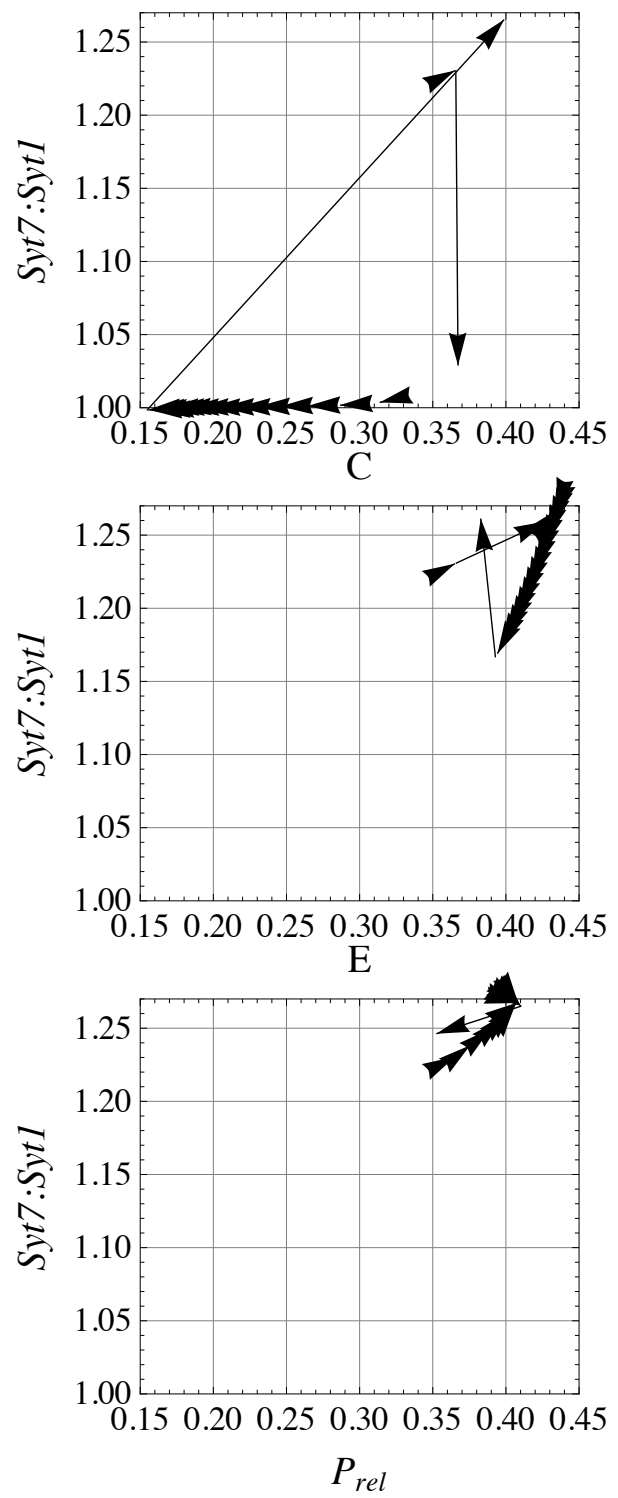

B
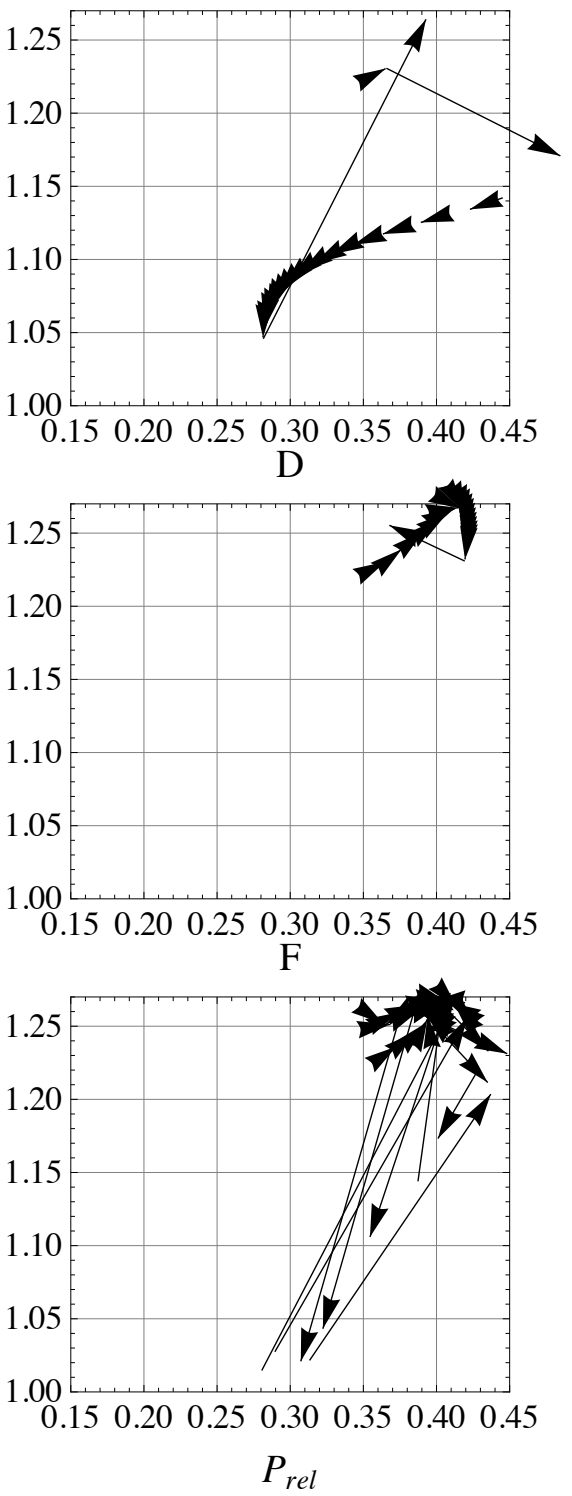

Figure 2.16. $P_{\text {rel }}$ vs. (Syt7 : Syt1) ratio at spike times in data set \#1. Arrows represent direction of time. A) $50 \mathrm{~Hz}$, B) $20 \mathrm{~Hz}$, C) $10 \mathrm{~Hz}$, D) $5 \mathrm{~Hz}$, E) $2 \mathrm{~Hz}$, F) complex train \#1. 

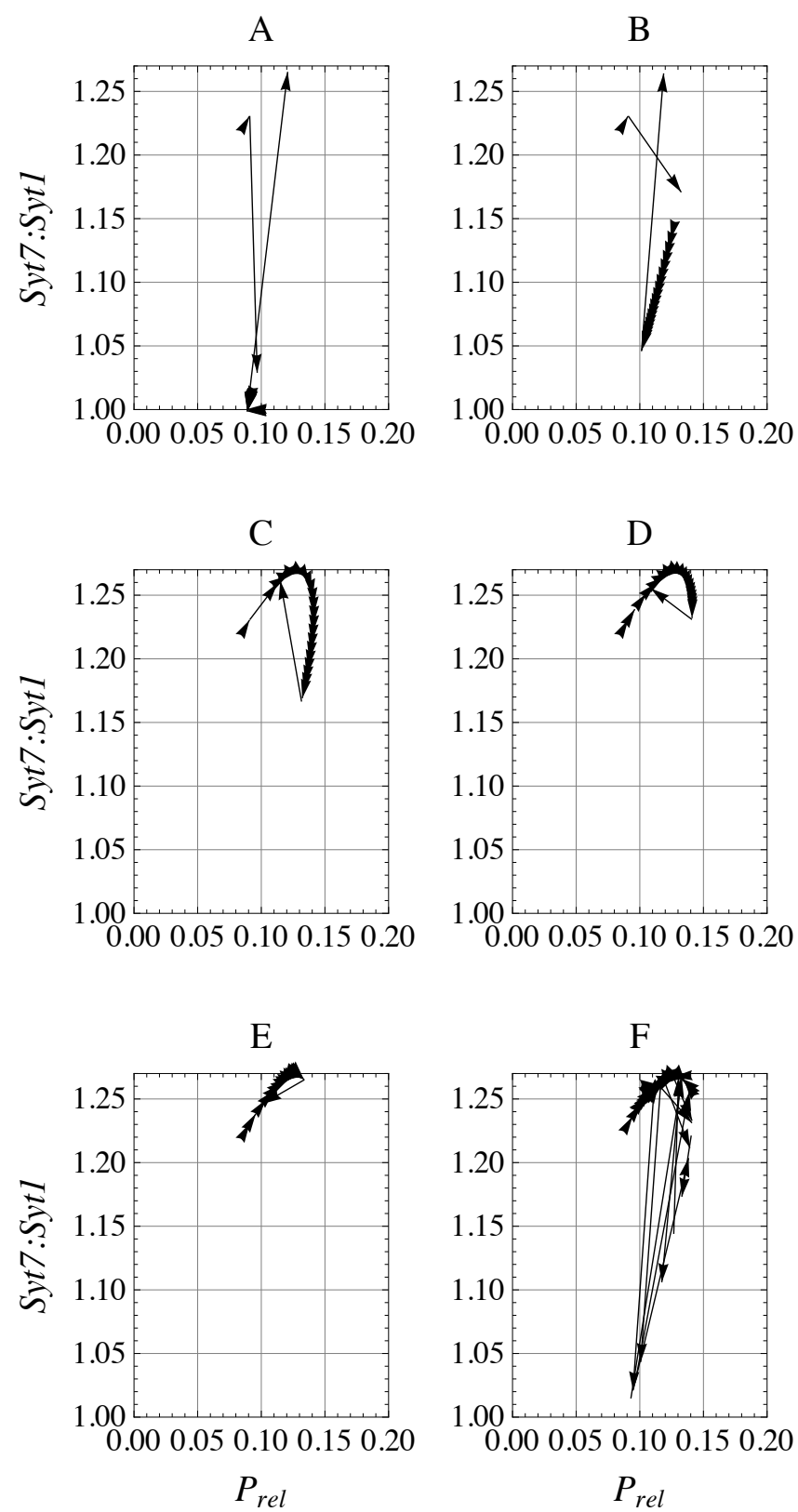

Figure 2.17. $P_{\text {ves }}$ vs. (Syt7: Syt1) ratio at spike times in data set \#1. Arrows represent direction of time. A) $50 \mathrm{~Hz}$, B) $20 \mathrm{~Hz}$, C) $10 \mathrm{~Hz}$, D) $5 \mathrm{~Hz}$, E) $2 \mathrm{~Hz}$, F) complex train $\# 1$. 


\subsubsection{Parameter sensitivities}

To assess the sensitivity of the model to changes in the parameters, the parameters were adjusted individually by $\pm 1 / 2 \%$ of their respective ranges, and the resulting changes behavior of the DSCI model observed. Variations from an unperturbed baseline release probability are shown Figs. 2.18-2.22. The parameters fitted to data set \#1 were used. ISIs from the training data were concatenated, including five of the constantrate trains (the $2 \mathrm{~Hz}, 5 \mathrm{~Hz}, 10 \mathrm{~Hz}, 20 \mathrm{~Hz}$, and $50 \mathrm{~Hz}$ trains) and one of the complex trains (\#1). The ISI information is removed, leaving only stimulus index of the concatenated stimulus trains. The divisions between stimulus trains are readily apparent in the figures. Red points are the response to $+1 / 2 \%$ relative changes in the parameters; black points are responses to $-1 / 2 \%$ relative changes. 

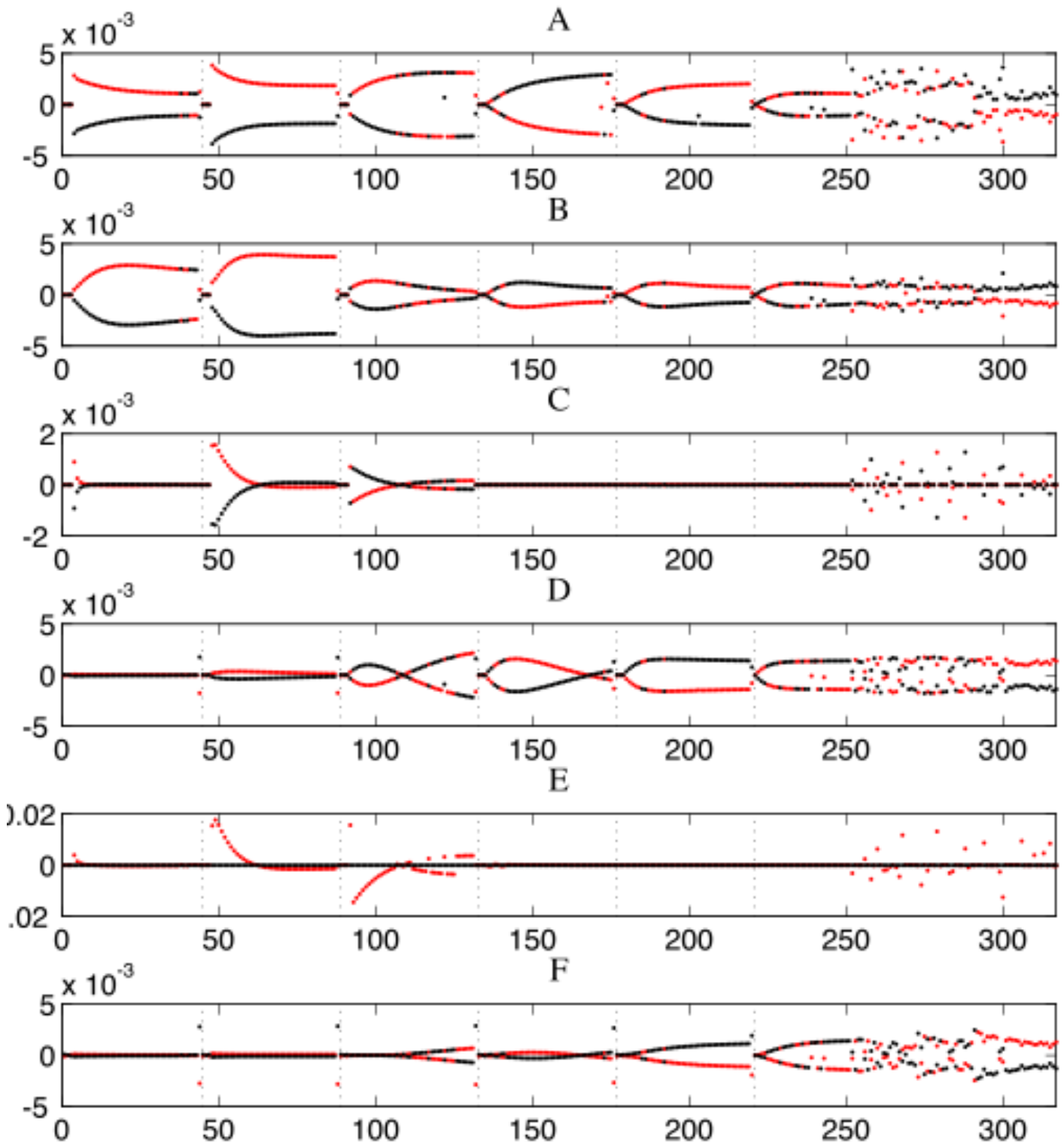

Figure 2.18. Parameter sensitivities $( \pm 1 / 2 \%)$. A) $\log \left[\mathrm{Ca}^{2+}\right]_{\mathrm{res}, 0}$. B) $\Delta_{\mathrm{Ca}, \mu}$. C) $\Delta_{\mathrm{Ca}, \text { int }}$. D)

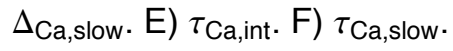



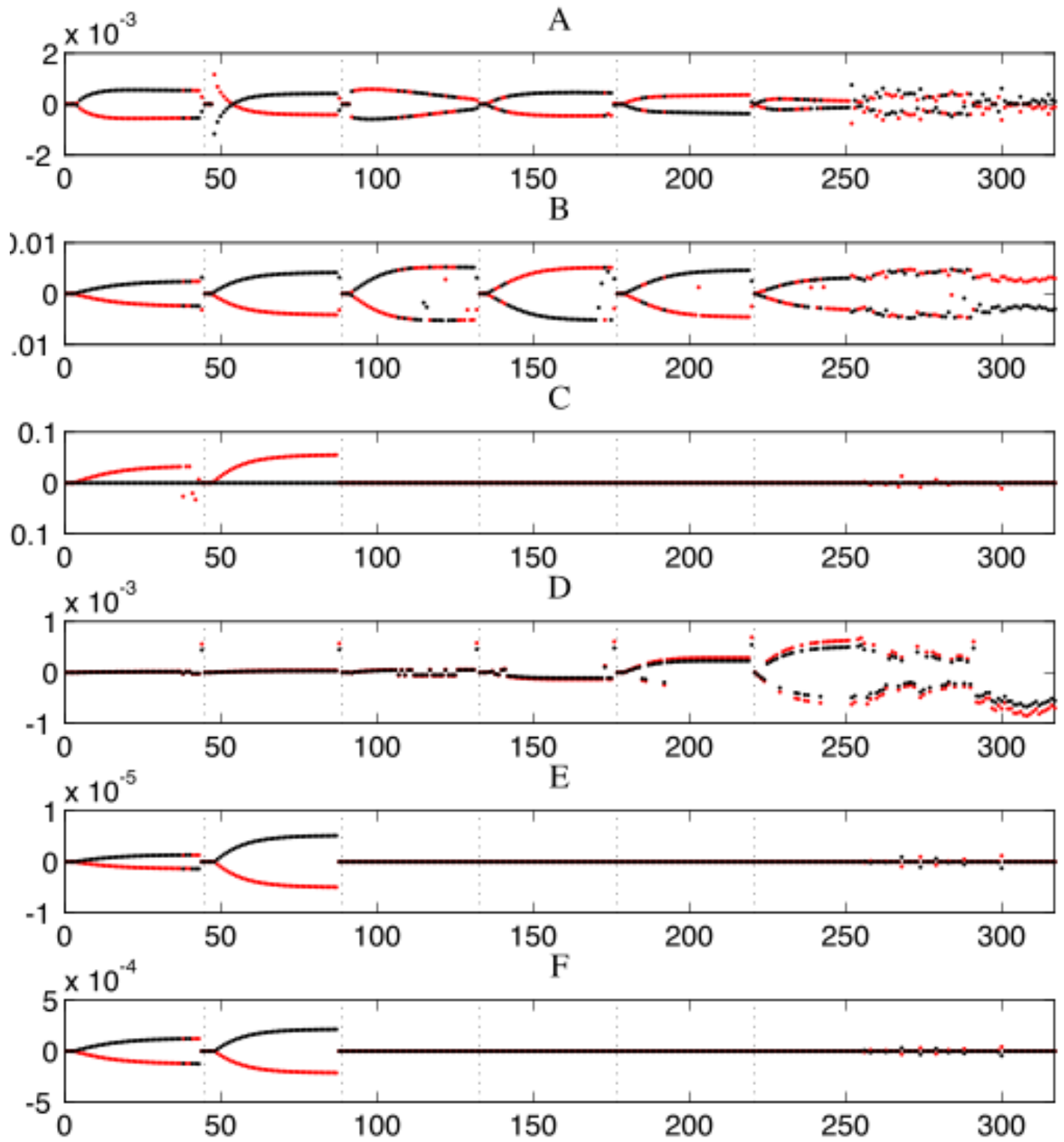

Figure 2.19. Parameter sensitivities $( \pm 1 / 2 \%)$. A) $n_{\text {rest. B) }} \phi_{n}$. C) $\tau_{\text {fast }}$. D) $\tau_{\text {slow. E) }}$ $\tau_{k \text {,refill }}$ F) Amp $_{\text {refill,fast }}$ 

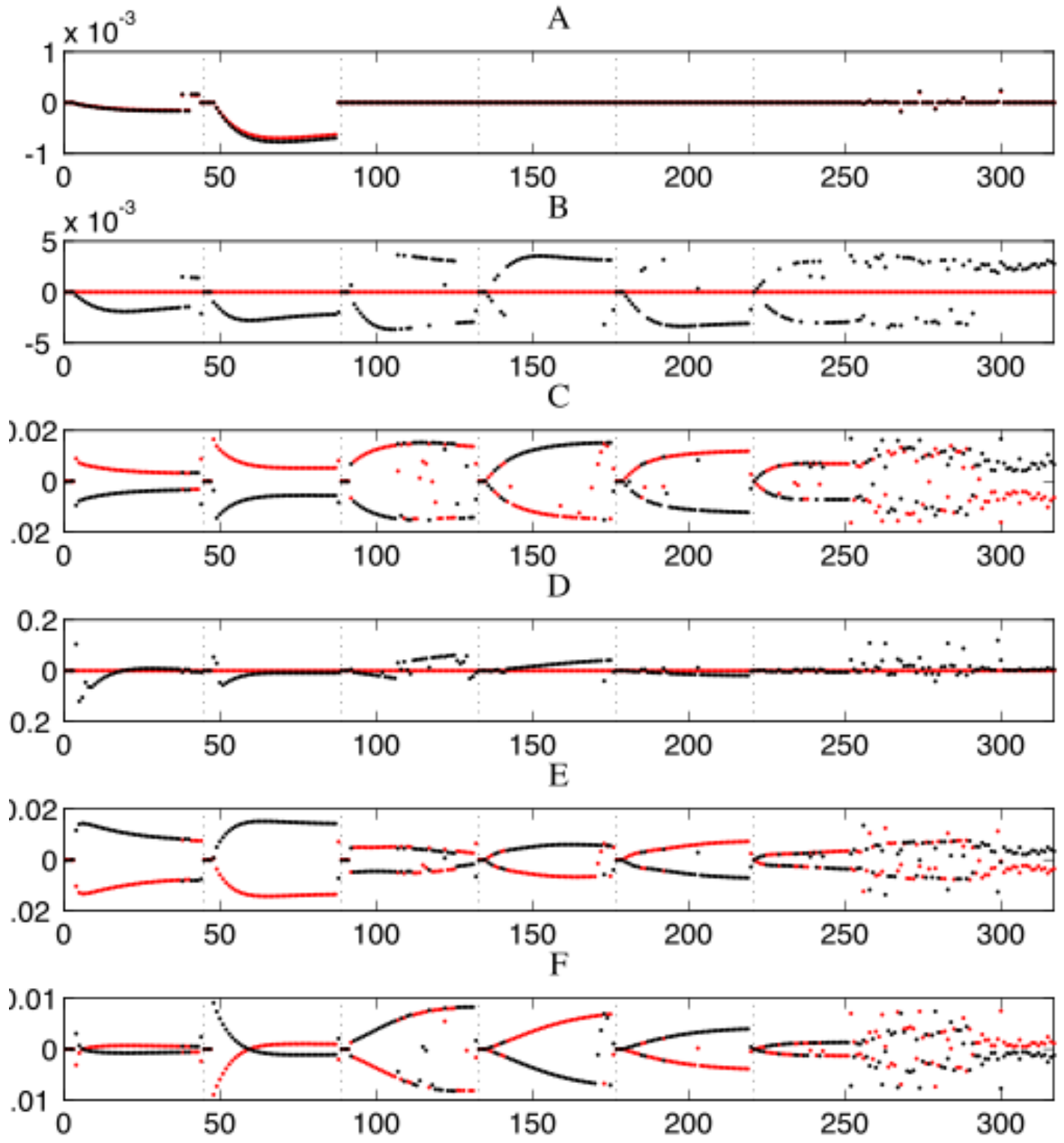

Figure 2.20. Parameter sensitivities ( $\pm 1 / 2 \%)$. A) $\mathrm{Kd}_{\text {refill,fast. }}$ B) $\phi_{\mathrm{Ca}, \mu} . \quad$ C)

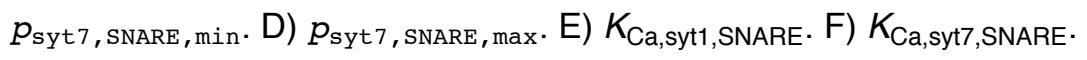



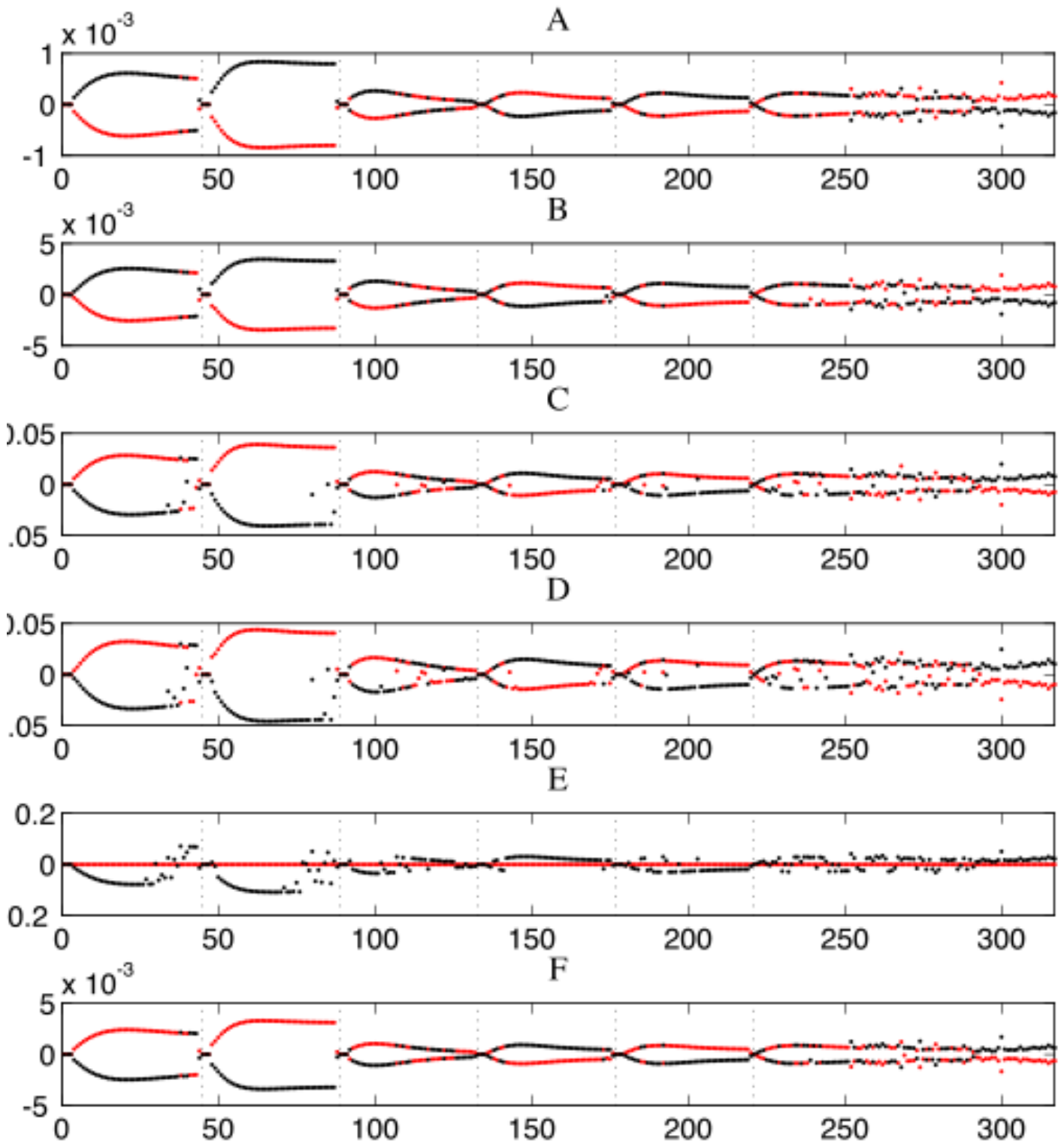

Figure 2.21. Parameter sensitivities $( \pm 1 / 2 \%)$. A) $K_{\text {Ca,syt1,trigger }}$ B) $K_{\text {Ca,syt7,trigger }}$ C) $E_{\text {syt. D) }} E_{\text {syt7 }}$. E) $N_{\text {SNARE. }}$ F) $n_{\text {Ca, syt1,trigger }}$. 
A
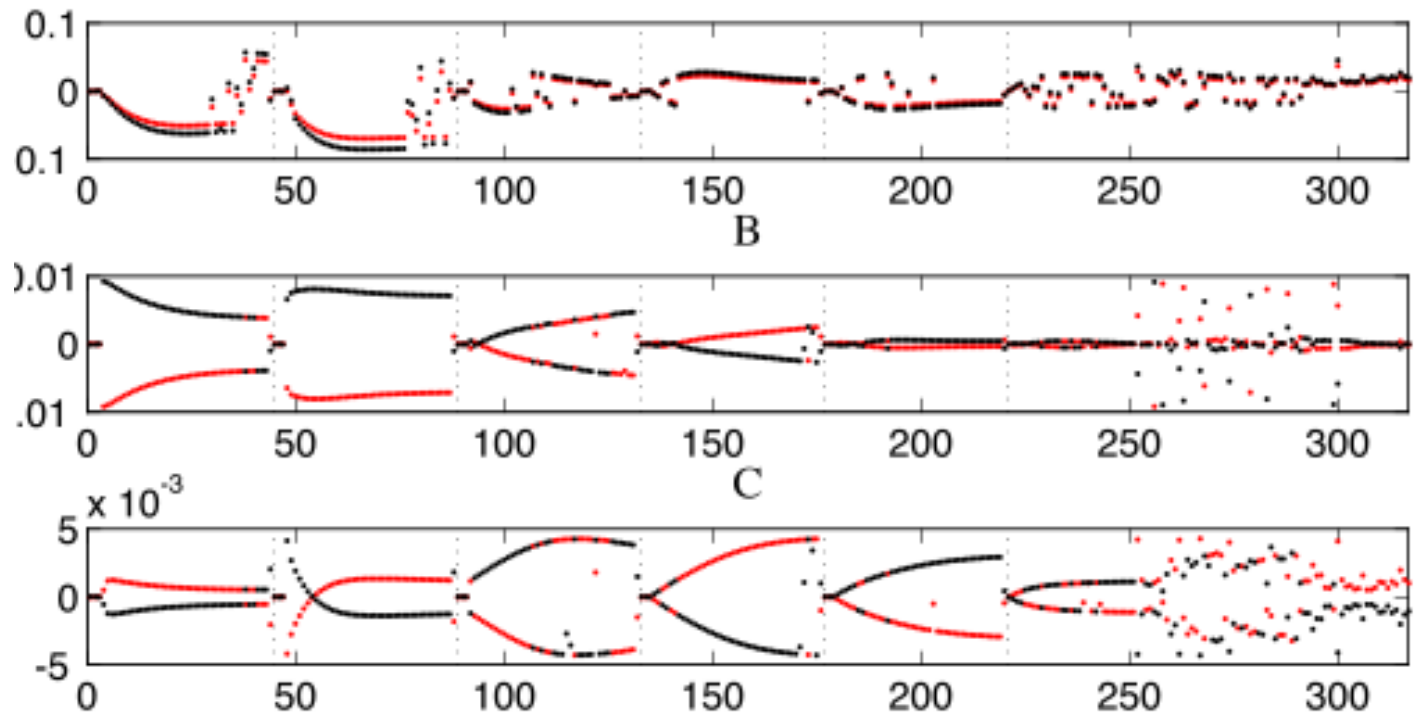

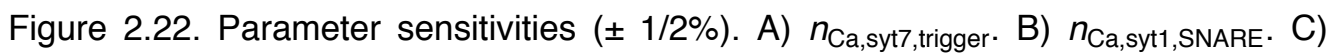
$n_{\text {Ca,syt7,SNARE. }}$

\subsubsection{Pharmacological verification (potential)}

Pharmacological treatments corresponding to individual parameters in the DSCI model could be applied to evaluate the predictive power of the model. An example is 4aminopyridine (4-AP), a potassium channel blocker and calcium channel activator [Wu et al, 2009; Hjelmstad, et al, 1997]. By blocking the potassium channel, 4-AP allows the sodium channel to dominate the membrane potential, increasing the width of the action potential. In combination with the direct activation of calcium channels, this allows the cytosolic calcium concentration to saturate the buffer and sensor mechanisms. The DSCI model could be used to predict the behavior of synaptic dynamics in response to a saturating calcium signal by increasing the three calcium update parame- 
ters, $\Delta_{\mathrm{Ca}, \mu}, \Delta_{\mathrm{Ca}, \text { int }}$ and $\Delta_{\mathrm{Ca} \text {,slow }}$. As a first approximation, these three parameters could be increased by an amount that is linearly proportional to the increased spike width.

\subsubsection{Unaccounted-for features}

The DSCI model seems less able to describe some of the lower-frequency facilitative behavior, especially in the constant-rate trains. The DSCI model also does not attempt to describe post-tetanic potentiation (PTP), so it is not surprising that there is large error at these points. The DSCI model cannot completely describe the late-train depression seen in the complex train used during training (see Fig. 2.7.F, last 30 points). Finally, the model cannot accurately track the rate of depression seen in the high-frequency constant rate data (Fig. 2.7.A and $B$ ).

\subsection{Predictions of the DSCI model}

What does the DSCI model indicate about the phenomena lurking behind the assump tions used to formulate the model?

\subsubsection{The molecular hypothesis}

By eliminating the difference in peak calcium concentration experienced by willing and reluctant vesicles (by driving $\phi_{\mathrm{Ca}, \mu} \rightarrow 0$ ), the model supports the molecular hypothesis, or the idea that vesicle proximity to calcium channels doesn't fully explain synaptic dynamics [Wolfel et al, 2007]. It is important to bear in mind that the molecular hypothesis is not a model of STP, so the DSCI model is unique from the molecular 
hypothesis in describing STP processes.

\subsubsection{The distinction between vesicle pools}

The ratio of willing to reluctant vesicles, $\phi_{n}$, changed remarkably little in the fit to the second data set, even though the total number of vesicles changed by a large amount. The fit is more sensitive to the fraction of willing to reluctant vesicles than to the number of vesicles, but probably because the gain is normalized. The difference in refilling rates between the vesicles pools was also exploited by the model. The optimiza tion of the error function essentially made the refilling rates as different as the bounds would allow. It might seem that the fit was trying to get rid of the reluctant pool. However, the fit retained a remnant of the reluctant pool to contribute to depression via vesicle depletion. The reluctant vesicle depletion doesn't account for the late-train depression seen in the first of the two complex data trains, however.

\subsubsection{The release machinery}

Due to the difference between the contributions to the energy of membrane fusion between the primary and secondary calcium sensors (syt1 and syt7) in the DSCI model, the DSCI model can't match the syt1 knock-down results of [Xu et al, 2012]. Even though the results of $\mathrm{Xu}$ et al include asynchronous release, the synchronous charge transfer still exceeds what the current DSCI parameters would predict by at least an order of magnitude. The discrepancy between the DSCI model and the results of syt1 knock-down indicate another constraint in the parameters that should be 
included in the optimization stage.

\subsection{Summary}

A mechanistic model of STP has been introduced that displays enough generality to describe concurrent facilitative and depressive synaptic dynamics. This is the first mechanistic model of STP capable of describing both facilitation and depression simultaneously, and should prove to be a useful tool for exploring hypotheses about synaptic functionality.

The new model of STP is based on contemporaneous observations of the proteins responsible for synaptic vesicle exocytosis. In particular, the understanding that there are multiple calcium-sensing release-triggering proteins, provided the inspiration for a description of neurotransmitter release probability based on the competitive interaction between calcium sensors for binding-site occupancy on the release machinery. The calcium-dependent efficiency of the release machinery to stimulate membrane fusion has been described in terms of the binding affinity of the calcium sensors to the release machinery and the calcium affinity of the release-triggering conformational change of the calcium sensors.

Insight into the neurotransmitter release process provided by the new model of STP may result in the ability to ascribe characteristics of synaptic dynamics to the constituency of calcium sensors at the synaptic active zone. This understanding could result in the development of pharmaceuticals that are targeted to correct cognitive deficits caused by alterations to the dynamic properties of synaptic transmission. 
C H A P T E R 3

\section{Characterizing STP at the Schaffer collateral synapse}

Given the physiologically realistic DSCI model of the synaptic dynamics at the Schaffer collateral, the nature of the processing at the Schaffer collateral is examined in this chapter. Two hypotheses designed to elucidate the function of STP at the SC are presented and tested. The hypotheses are intended to assess whether SC synaptic dynam ics favor certain signal characteristics, and if so, how the CA1 neuronal output is affected. Statistical variation in the signals carried on the Schaffer collateral are observed in the distribution of ISIs. The simulations reported here are considered from the perspective that STP behaves like a filter tuned to expected ISI distributions. From this perspective, the characteristics of the STP filter can be understood in the context of the operational regimes of the hippocampus.

\subsection{Preliminary considerations}

\subsubsection{Input sources}

Input spike trains were generated within a statistical framework. First, spike times were drawn from a probability distribution, then additional variation, correlation, or modulation was imposed upon the spike trains. In a manner similar to [Frerking et al, 
2005], spike trains were created having end-to-end segments of arbitrary length, each segment having its own properties (mean rate, number of correlated trains, correlation coefficient, modulation, etc). Multiple spike trains, not necessarily having the same length or number of spikes were generated in this way to simulate inputs at individual synapses. With enough inputs, the average rate of the inputs on any given segment approached a constant value. Figure 3.1 shows the distribution of cumulative ISIs for 15 seconds, with 75 input trains drawn in three five-second segments at mean rates of $1 \mathrm{~Hz}, 5 \mathrm{~Hz}$ and $2 \mathrm{~Hz}$ from the empirical distribution of [Frerking et al, 2005] (see Fig. 3.3 below). Figure 3.2 shows the rastor plot for the 75 inputs.

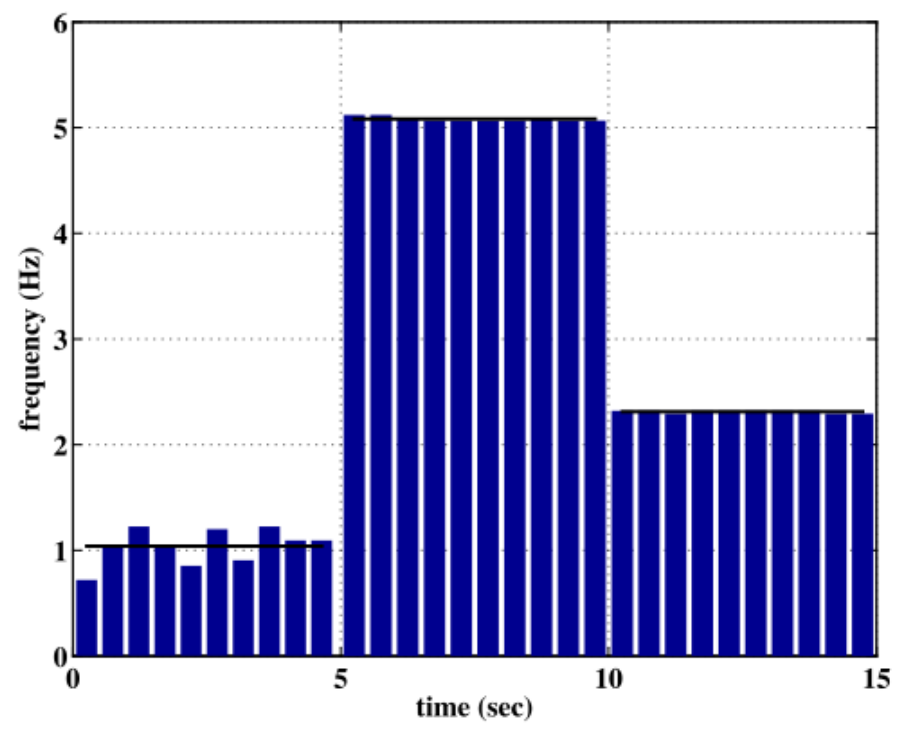

Figure 3.1. Histogram of the spike times for a sample input having 75 spike trains in three segments: $1 \mathrm{~Hz}$ for $5 \mathrm{sec}, 5 \mathrm{~Hz}$ for $5 \mathrm{sec}, 2 \mathrm{~Hz}$ for $5 \mathrm{sec}$. ISls drawn from the empirical distribution in [Frerking et al, 2005]. Compare to Fig.11B. in [ibid]. Black lines above bars show the mean frequency of inputs in each segment. 


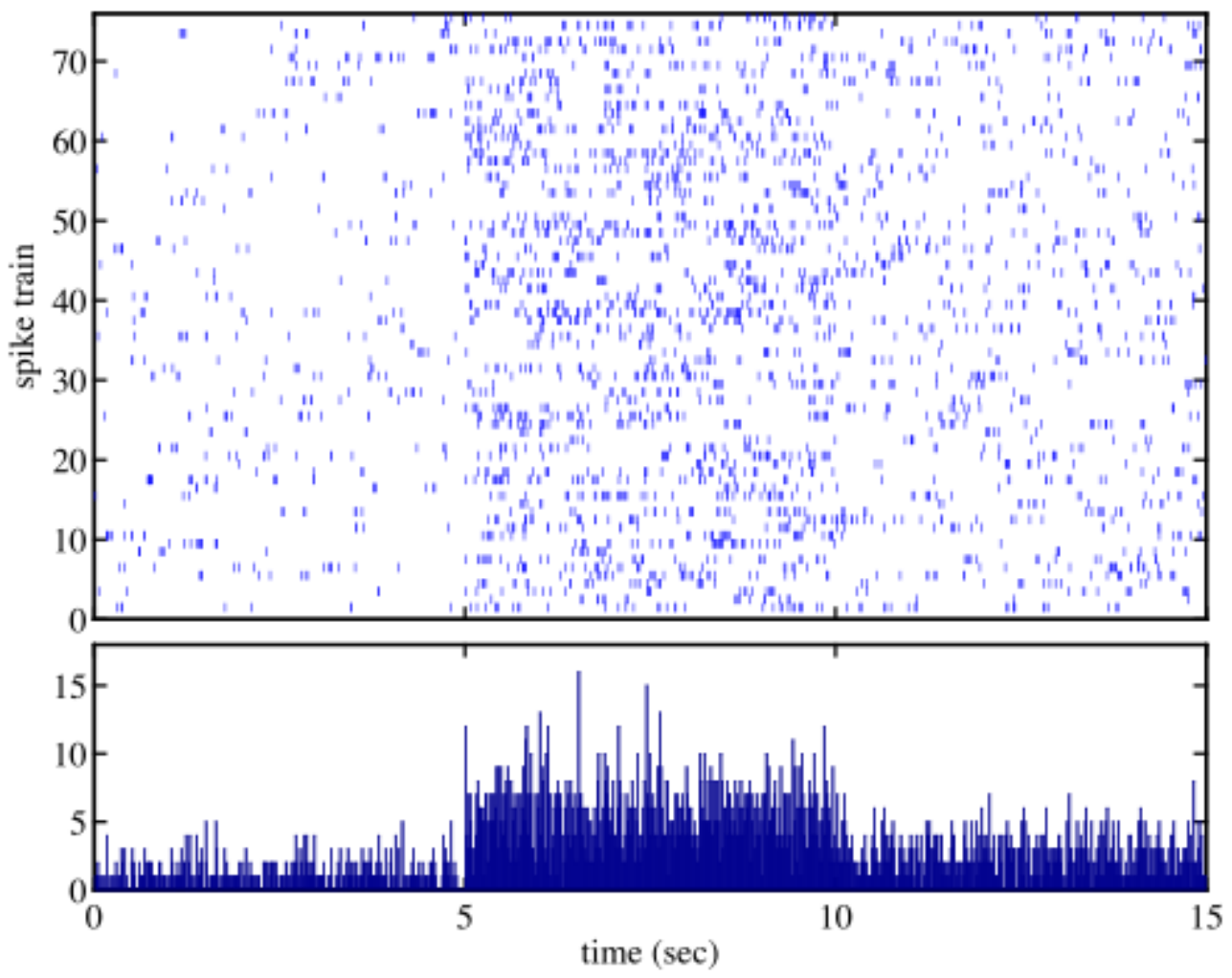

Figure 3.2. Rastor plot and histogram of the spike times for a sample input having 75 spike trains in three segments. Input scenario described in Fig. 3.1.

\section{Empirical ISI distribution}

Spiking signals were recorded from the CA3 of awake and behaving animals exposed to two different stimuli: an odor cue and a spatial cue [Frerking et al, 2005]. Nonrandom spike sequences were observed, yet it was found that the ISI probability distributions of the responses were the main factor in generating the nonrandom spike sequences. Because the majority $(\approx 90 \%)$ of the ISI variance could be described by samples drawn randomly from a probability distribution, it was proposed that an empiri- 
cal probability distribution describing the average behavior of CA3 cells would be useful for generating spike trains for studies of synaptic physiology, especially by alleviating the problem of introducing non-representative activity patterns that may arise when actual spike trains are used as stimuli [Frerking et al, 2005]. ISI probability distributions were collected having mean rates within about $0.2 \mathrm{~Hz}$ and $8 \mathrm{~Hz}$, compiled and interpolated to create the continuous function of ISI distributions vs. mean frequency [ibid] shown in Fig. 3.3.

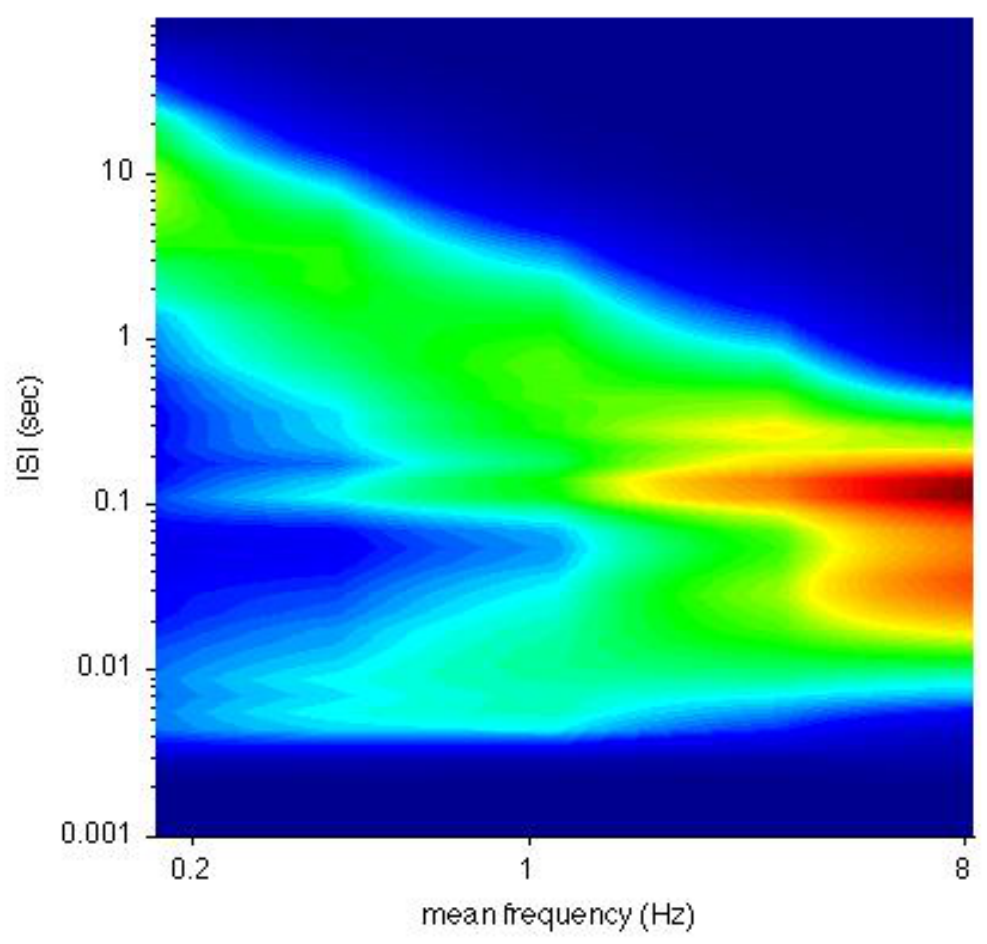

Figure 3.3. Contour plot of the [Frerking et al, 2005] empirical ISI probability distribution $($ red $=0.075$, blue $=0$ ). 


\section{Sources of variation}

Variation was introduced to the input signals in several forms. A physiological source of stochastic background synaptic currents [Destexhe et al, 2001] was added during numerical integration of the CA1 neuron (See Appendix 4 for details). Background current fluctuations were estimated to account for as much as $80 \%$ of the total conductance of a cortical pyramidal cell [Destexhe and Pare, 1999]. If the entorhinal cortex (EC) current source to the CA1 is assumed to be mostly uncorrelated, then the Destexhe current could serve as an estimation of the EC inputs. Like the EC inputs, the background current fluctuations are not sufficient to invoke spiking, but can influence subthreshold behavior, which can modulate the probability that the cell will fire. Since inputs from the EC can be modulated (for instance, by local field potential oscillations - see Ch. 4), the model of stochastic background currents was given an optional sinusoidal modulation.

The other sources of variance affected only the correlation and jitter of spikes from the CA3. The correlation of signals from the CA3 was simulated by a spike-swapping procedure [Niebur, 2007]. Using the Niebur procedure, a controlled percentage of input trains could be correlated with a reference train to a specified degree. Additionally, spike trains could optionally receive oscillatory modulation of fixed phase and frequency, representing, for instance, fast gamma modulation from the CA3. Spike trains could also be decorrelated by randomly jittering the spike times. The added random jitter was implemented with a Gaussian process (random samples drawn from a normal distribution). 


\subsubsection{A model CA1 pyramidal cell}

A two-compartment model of a CA1 pyramidal cell was used to examine the effect of STP on the output of an excitatory neuron. The CA1 model is a modification of a well-known CA3 model [Pinsky and Rinzel, 1994]. The modification attenuates the characteristic CA3 bursting behavior by reducing the conductance of the slow dendritic calcium current. The CA1 model is described in Appendix D.

\subsubsection{Spike train measures}

The "Law of Requisite Variety" [Ashby, 1956] states (paraphrasing): 'A system's response to variety in the states of its inputs is limited to the variety of the system's internal states'. The variety of a neuronal response exists (in large part) in the pattern of timing between spikes. Quantifying the variety in spike trains is the subject of this section.

\section{Rate}

The number of spikes in a period of time, $\tau$, is a measure of neuronal activity [Dayan and Abbott, 2001]. However, determining which period of time should be used is controversial [Rieke et al, 1999]. In the limit of $\tau \rightarrow 0$, the precise timing of spikes is assumed to be critical for signal information transfer, an assumption unlikely in all but the largest synapses (such as the calyx of Held). In the limit, $\tau \rightarrow \infty$, the rate of spikes is assumed to carry behavioral significance, requiring unrealistic integration times. These 
two limits represent a dichotomy within the neuroscience community, and the Truth probably lies somewhere in-between. For instance, some neural processes suggest that precise spike timing is important (for instance, spike timing dependent plasticity and phase modulation), while the overwhelming presence of noise in the response of a neuron suggests otherwise [Frerking, personal communication; Liaw and Berger, 1999]. The average spiking rate, and the spike rate vs. time may both be important for transmitting information over spike trains.

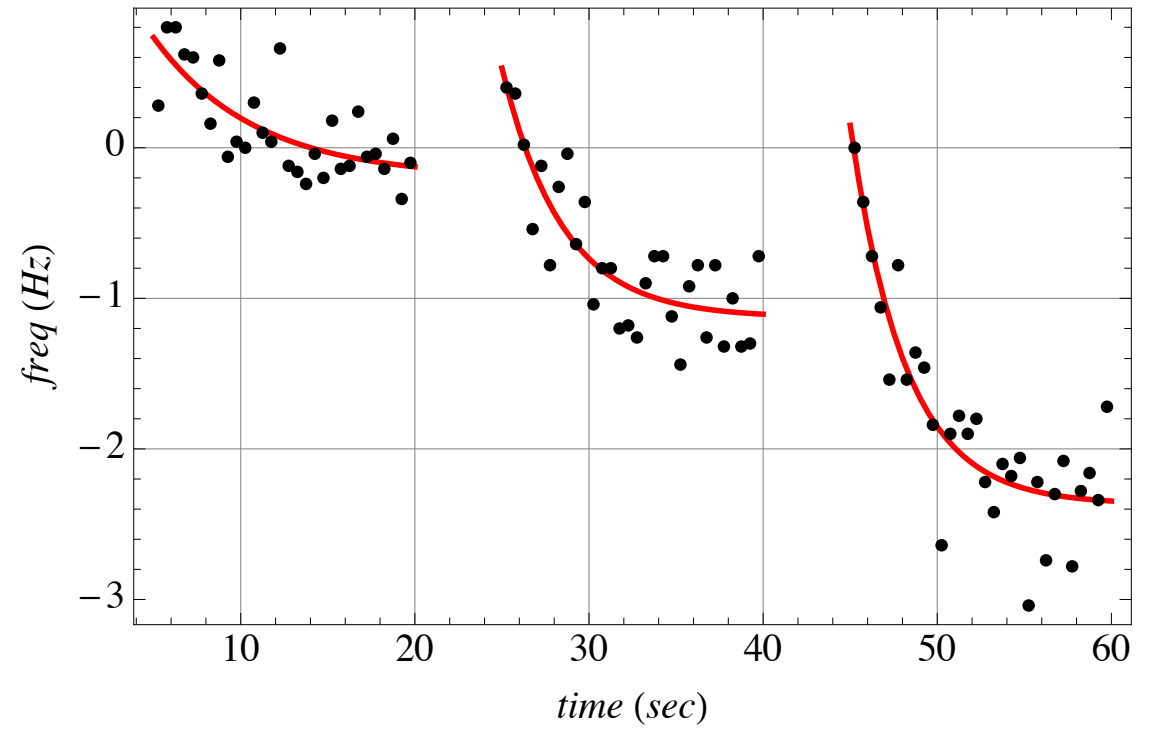

Figure 3.4. CA1 model output spike rate vs. time response (STP - nonSTP) for DSCI model \#1. Input rates shown in Fig. 3.6. All inputs drawn from empirical CA3 ISI distribution (Fig. 3.3). 


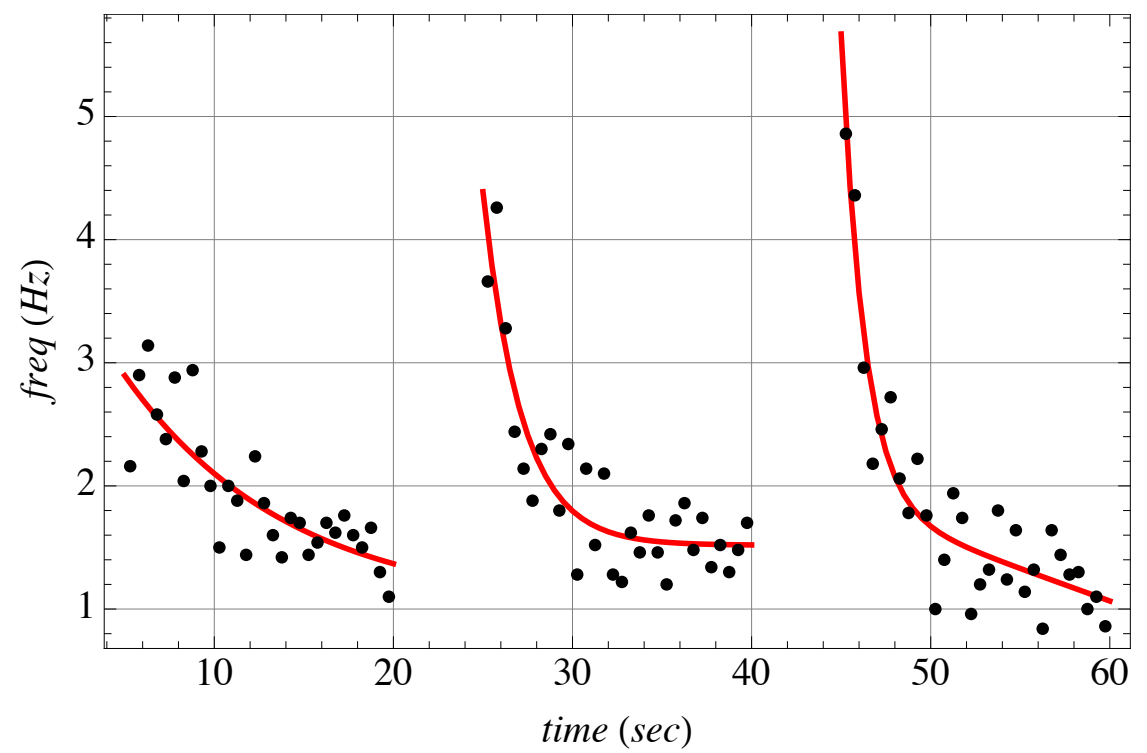

Figure 3.5. CA1 model output spike rate vs. time response (STP - nonSTP) for DSCI model \#2 (Input rates shown in Fig. 3.6). All inputs drawn from empirical CA3 ISI distribution (Fig. 3.3).

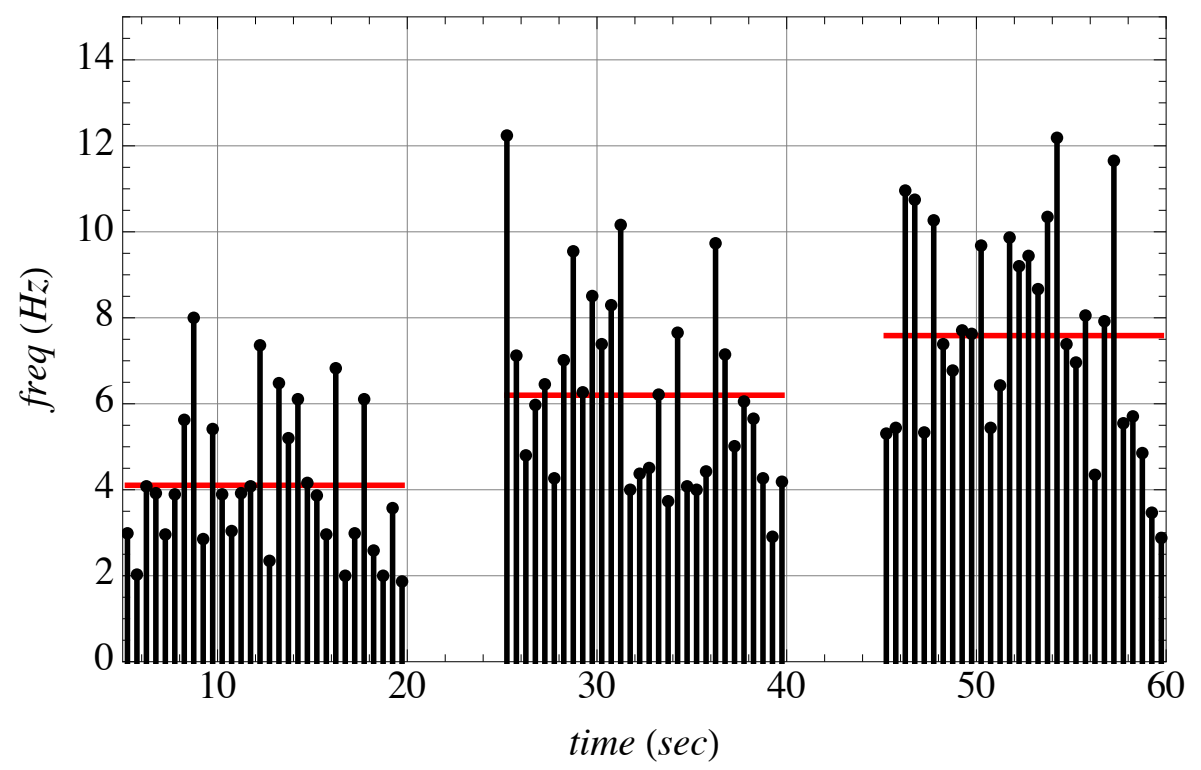

Figure 3.6. Input rates for responses shown in Figs. 3.4 and 3.5. 


\section{Information}

Entropy is related to the probability, $p_{i}$, of observing a particular state, $i$, in a sample taken randomly from a set of states that includes state $i$. Entropy, $S$, is defined as:

$$
S=-\sum_{i} p_{i} \log _{2} p_{i}, 0 \leq p_{i} \leq 1 \forall i, \text { and } \sum_{i} p_{i}=1
$$

An entropy measure for spike trains has been developed [Strong et al, 1998]. The Strong entropy considers the observable states to be the possible binary words consisting of concatenated spike states (spike or no spike) within a time window, $T$, discretized into bins of width $\Delta \tau$. For example, one of the $2^{10}$ possible binary words in a one-second window discretized into $100 \mathrm{msec}$ bins, is “0100100001", where "1" represents at least one spike in the $100 \mathrm{msec}$ bin, and "0", no spike. The entropy of interest occurs in the limit of $T \rightarrow \infty: S(\Delta \tau)=\lim _{T \rightarrow \infty} S(T, \Delta \tau) / T$. Since this limit is never achievable in practice, an approximation was developed by Strong et al, in which (presumably by the principal of ergodicity) shorter time windows can be used to obtain a reliable estimate of $S$. By averaging over multiple presentations of the same stimulus, an estimate of the variability in the response, called the "noise entropy", can be subtracted from the total entropy to obtain a value for the information transmitted by the cell.

\section{Distance}

Spike trains may be considered as points in a high-dimensional space. Naturally, some measure of distance between spike trains may then be defined. Several distance measures have been proposed for spike trains [Victor and Purpura, 1997; van Rossum, 
2001; Szucs, 1998]. The van Rossum measure is distinctive because it has a physiological interpretation. The van Rossum distance assumes that spikes influence other spikes through the postsynaptic potential (PSP) [van Rossum, 2001]. The influence of a PSP can be estimated by an exponentially decaying function that represents the activity of postsynaptic receptors, such as AMPA or NMDA, that produce an extended response to a spike. The van Rossum distance is defined by:

$$
D^{2}(f, g)_{t_{c}}=\frac{1}{t_{c}} \int_{0}^{\infty}[f(t)-g(t)]^{2} d t,
$$

where $f(t)$ and $g(t)$ are the two spike trains, convolved with a decaying exponential function:

$$
\begin{aligned}
& f(t)=\sum_{i=1}^{M} \Theta\left(t-t_{i}\right) e^{-\left(t-t_{i}\right) / t_{c}} \\
& g(t)=\sum_{i=1}^{N} \Theta\left(t-t_{i}\right) e^{-\left(t-t_{i}\right) / t_{c}} .
\end{aligned}
$$

$\Theta(\cdot)$ is the Heaviside step function, $M$ and $N$ are the number of spikes in trains $f$ and $g$, and $t_{i}$ are the spike arrival times.

\subsection{STP transfer function}

\subsubsection{Constant-rate stimuli}

The effect of the DSCI model of STP was characterized by response both to constant-rate stimuli, and to variable-rate stimuli. The gain curves of the DSCI model were characterized for both parameter vectors listed in Table 2.4. For constant-rate inputs between $0.5 \mathrm{~Hz}$ and $50 \mathrm{~Hz}$, the PPF, maximum gain, steady-state response, and maximal 
depression were determined. 1000 stimuli were applied to determine the asymptotic steady-state response. As in the constant-rate data, three stimuli at $0.1 \mathrm{~Hz}$ were presented prior to each of the input spike trains. Constant-rate results are shown in Fig. 3.7.

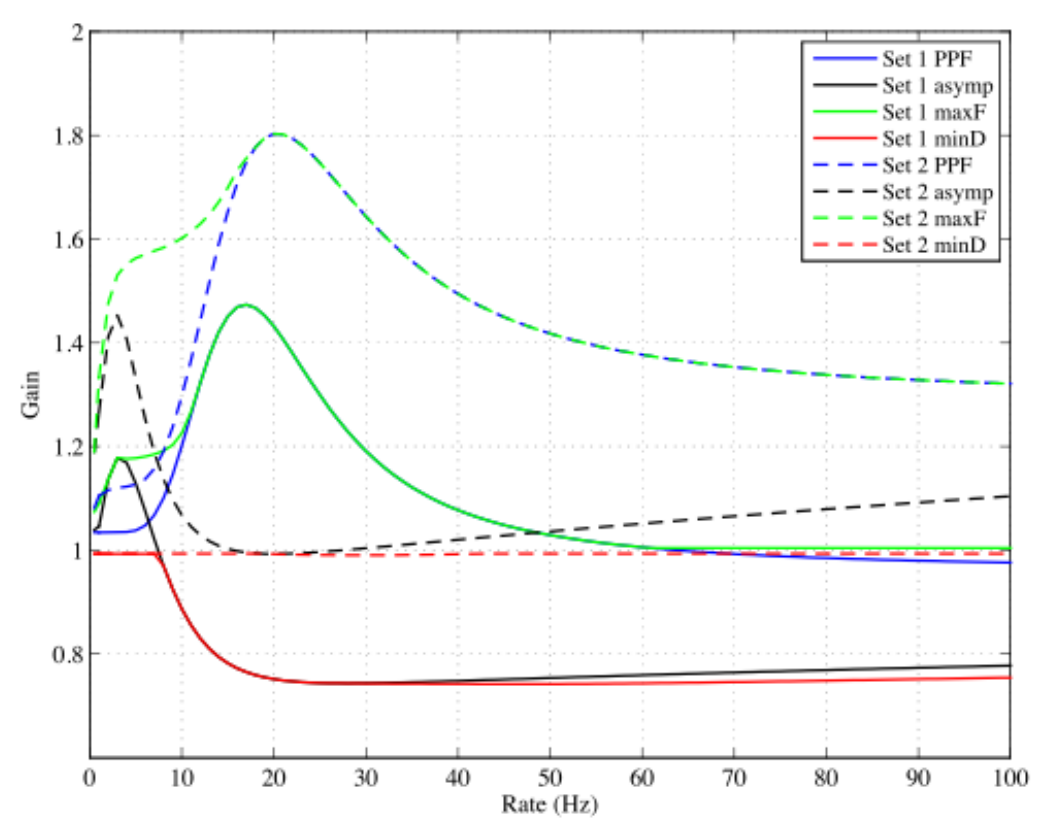

Figure 3.7. Gain response of DSCI STP to constant-ISI inputs. STP models (see text) are distinguished by solid and dashed lines.

\subsubsection{Variable-rate stimuli}

Synaptic gain vs. ISI for variable-rate stimuli are shown in Fig. 3.8. Input trains were generated from an empirical distribution of ISIs from CA3 cells that were responsive to test stimuli (see Fig. 3.3) [Frerking et al, 2005]. Responses of the two STP models to ISI trains with mean frequencies of $1 \mathrm{~Hz}$ are shown. The STP gains in Fig. 3.8 have been fit to the rescaled group delay of a six-pole filter. The choice to fit to the 
group delay was made to prove the point that the physiological meaning imparted by the spike code at the Schaffer collateral could be interpreted in more than one way. For instance, it is also possible to fit the data shown in Fig. 3.8 directly to the gain of a sixpole filter, in which case the spike code might signify the amplitude of the presynaptic signal. Section 3.3 explores the STP gain in response to variable-ISI inputs in more depth.
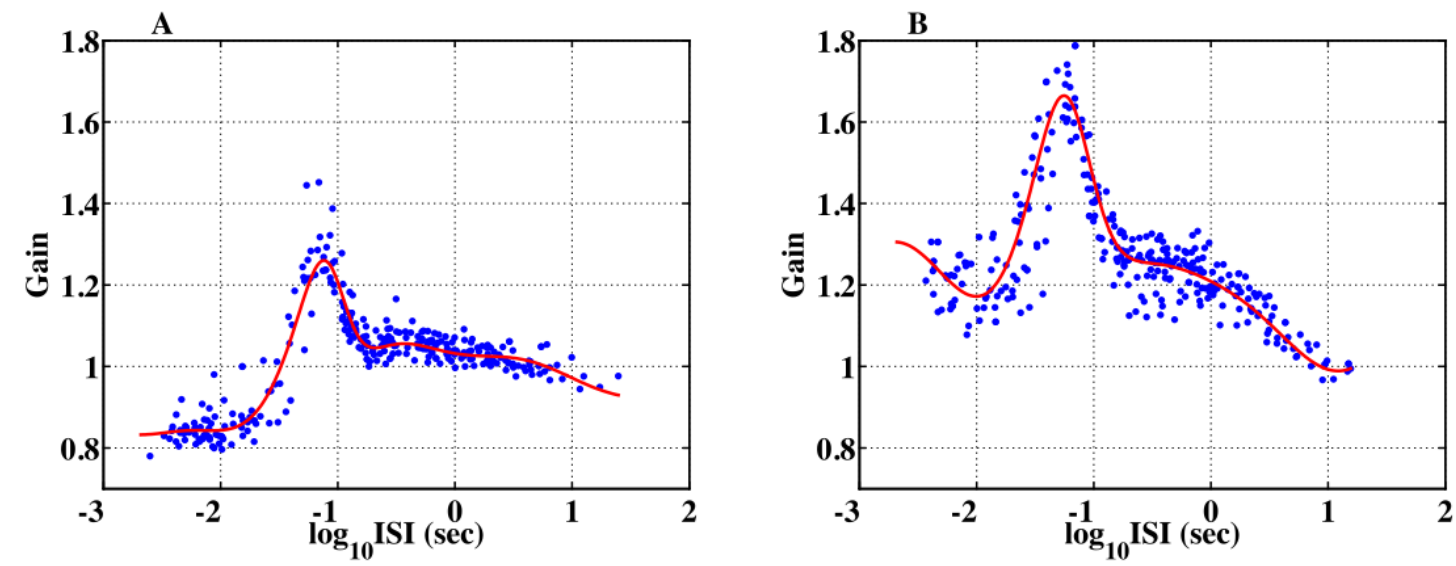

Figure 3.8. ISI-dependent DSCI STP gain for variable-ISI stimuli at $1 \mathrm{~Hz}$ mean rate. (A): Empirical CA3 distribution, DSCl model \#1. (B): Empirical CA3 distribution, DSCI model \#2.

\subsection{Effect of STP on Schaffer collateral processing}

Here, two specific hypotheses that address the central question about the effect of STP on Schaffer collateral processing, are presented and tested using the tools introduced above. 


\subsubsection{Hypothesis 1}

Input spike trains that have been generated from responsive CA3 ISI distributions

induce significantly different levels of facilitation when applied to the STP model than do spike trains generated from non-responsive CA3 ISI distributions.

\section{Test}

The difference between responsive and non-responsive CA3 spike trains is assumed to be largely reflected in the distributions of a representative sample from each (responsive and non-responsive) type of train. The empirical CA3 distribution [Frerking et al, 2005] is more representative of the responsive-cell population of ISIs (see Fig. 8 [ibid]). The non-responsive set must be approximated from the description given in [Frerking et al, 2005]. The reported difference between the two responses is a redistribution of energy from the middle part of the spectrum (centered around $5 \mathrm{~Hz}$ and extending from about $0.3 \mathrm{~Hz}$ to about $100 \mathrm{~Hz}$ ) to the extreme outer ends of the spectrum (centered at about $200 \mathrm{~Hz}$ and $0.1 \mathrm{~Hz}$ ) in the non-responsive set. To approximate the non-responsive set, the empirical CA3 distribution was sampled, then re-distributed in an approximation of Fig. $8 A_{3}$ [ibid] by adding scaled log-normal probability distributions and renormalizing. Figures 3.9-3.11 recapitulate Fig. 8, $A_{1-3}$ [ibid]. 


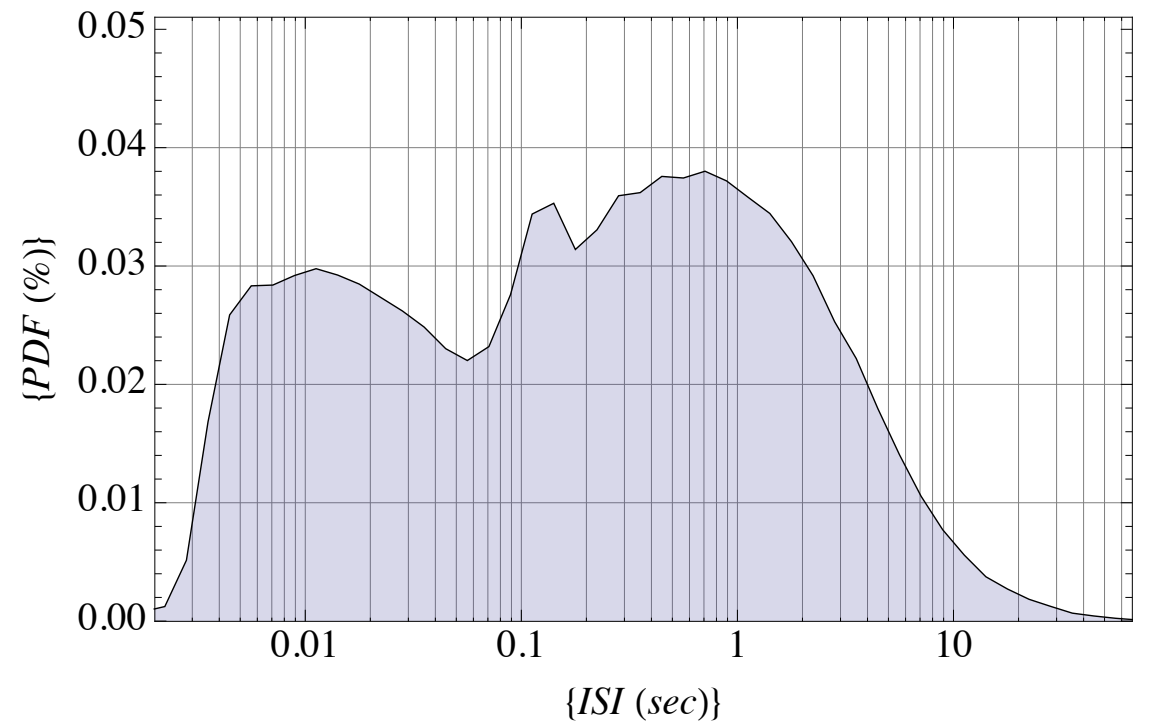

Figure 3.9. Responsive CA3 ISI distribution at $1 \mathrm{~Hz}$ mean rate (also see Fig. 3.3).

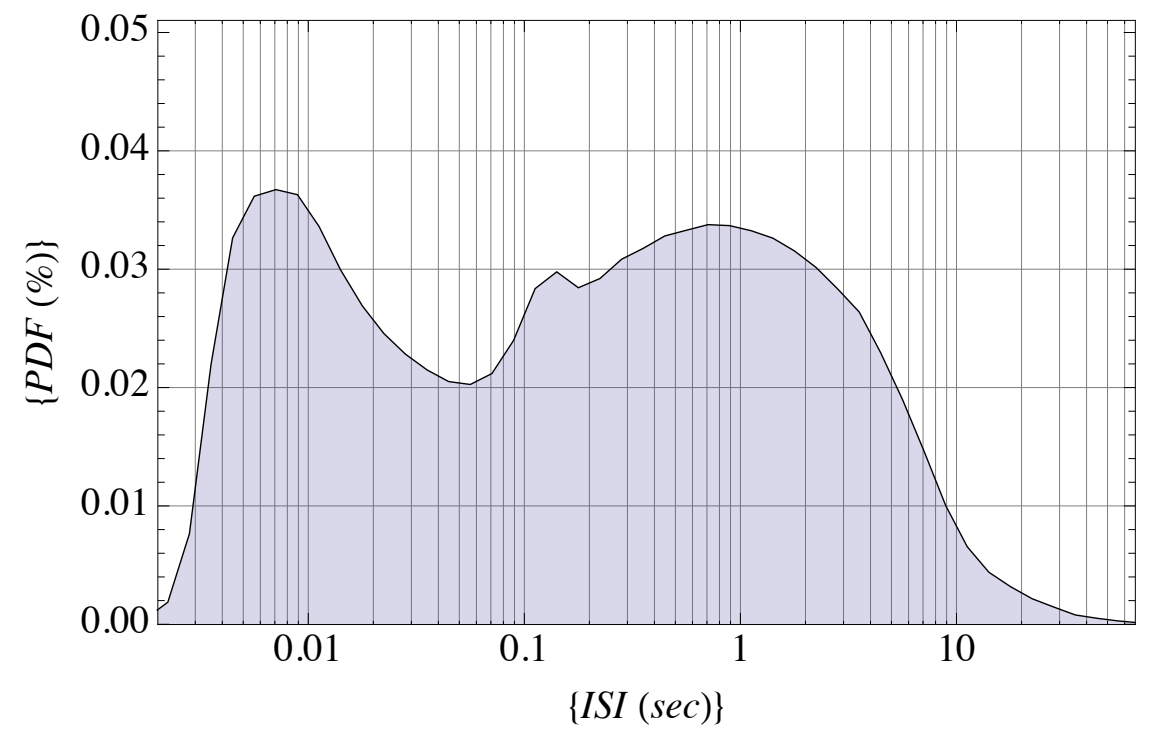

Figure 3.10. Approximate non-responsive CA3 ISI distribution at $1 \mathrm{~Hz}$ mean rate. 


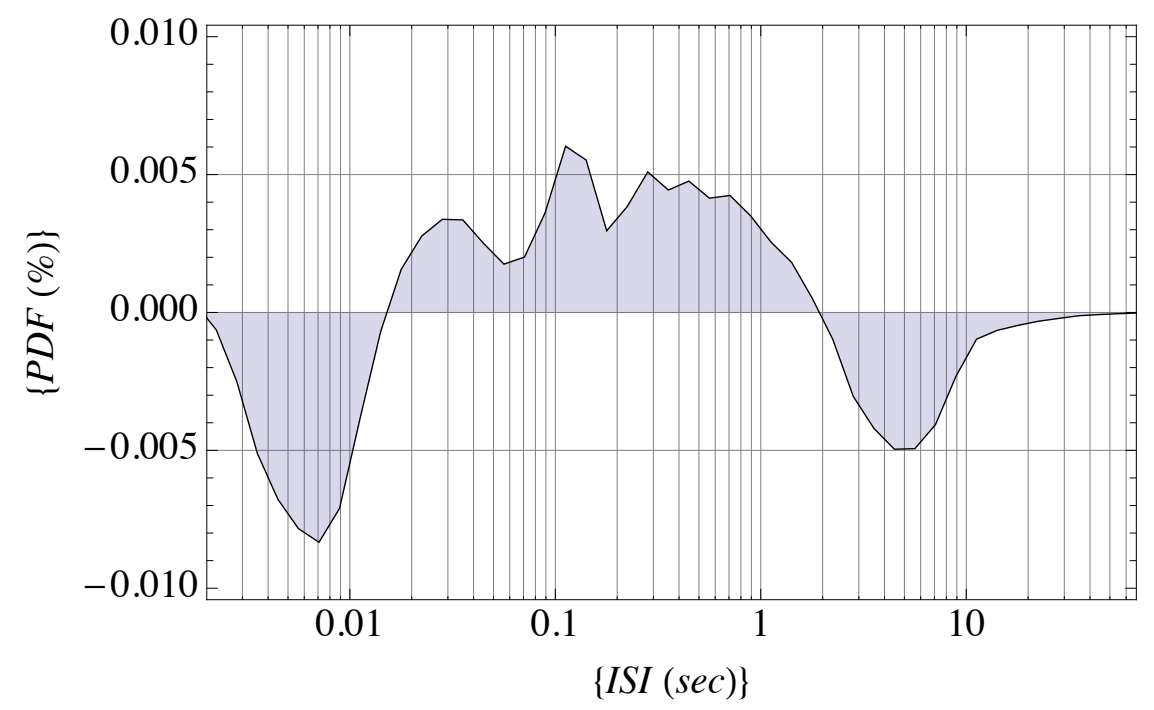

Figure 3.11. Difference of distributions shown in Figs. 3.9 and 3.10.

The meaning conveyed by the pattern of ISIs (must be) specific to the computational function of the specific microcircuit. In the CA3-CA1 circuit, one of the computational functions is to sequentially track the changes in the state of the recurrent CA3 network as the circuit reconstructs the causal expectations initiated by a set of cues from the DG and ECII. The sequential order of states is registered to the slow gamma $(20-40 \mathrm{~Hz})$ local field potential (LFP) generated by the CA3. For example, the position of the subject in an environment, as indicated by the activity of a place cell, $c_{0}$, in the CA3, is related to recalled paths through space (the activity of other place cells, $\left.c_{1}, \ldots, c_{n}\right)$ by the relative phase of the firing of $c_{0}$, with respect to theta $(5-12 \mathrm{~Hz})$ modulation of the LFP. The inital firing of place cell $c_{0}$ upon reception of the cue state late in the theta cycle precesses to earlier theta phase as the sequence of states in CA3 unfolds. From the reference point of a cell in CA1 receiving input from $c_{0}$, the meaning 
of the signal from $c_{0}$ is related to the transition of the states in CA3.

There are at least two ways to interpret the phenomenon of phase precession in terms of presynaptic filtering. Theta modulation may be considered as an envelope of the gamma signal, in which case the phase relationship of the gamma signal with respect to theta would be encoded in the group delay of gamma (the rate of change of the phase of gamma). In this case, STP might decode the signal from the CA3 in the domain of the group delay, as illustrated by the fits (red lines) in Fig. 3.8. It also possible to interpret the signal from the CA3 directly as a gain, in which case STP would behave like a filter in which the sampling rate is non-constant, while the amplitude of the samples is constant. Both alternatives were considered and found to provide equivalently good fits to the data.

Fitting the STP gain vs. ISI to filter characteristics went as follows: ISI samples were drawn from the responsive and non-responsive distributions, and 150-secondlong input spike trains were constructed from the sampled ISIs. Three hundred such 150 second-long input trains were applied to the DSCI model of STP for each distribution (responsive and non-responsive) at mean sampling frequencies of $1 \mathrm{~Hz}, 2 \mathrm{~Hz}$ and $3 \mathrm{~Hz}$. The synaptic gain values were paired to their immediately preceding ISI values. Samples from the resulting ISI $^{-1}$ vs. gain curves (see Fig. 3.8) were then fit to both the group delay and the gain of a six-pole discrete filter, the transfer function of which is given by (in the $z$-domain): 


$$
H(z)=\frac{Y(z)}{X(z)}=\frac{\beta_{0}+z^{-1} \beta_{1}+\ldots+z^{-1} \beta_{6}}{\alpha_{0}+z^{-1} \alpha_{1}+\ldots+z^{-1} \alpha_{6}},
$$

where $z=A e^{i \omega}$. The gain of the transfer function is given by the magnitude, $G(z)$, of $H(z): G(z)=|H(z)|$. The group delay is defined as the delay of the modulation envelope of a frequency component of a signal: $\tau_{g}=-\frac{d \phi(\omega)}{d \omega}$, where $\phi(\omega)$ is the phase response of the filter, defined as: $\phi(\omega)=\arg \left(H\left(e^{i \omega}\right)\right)$. An exploration of the filter charac teristics for the two interpretations indicate that the fit to the group delay directly yields a linear phase reponse. However, it is uncertain what the meaning of the gain is in this interpretation. The magnitude and phase response from the group delay fit are shown in Fig. 3.12. The filter is not minimum phase, but is stable. Figure 3.13 shows an example filter, the gain of which was used to fit the STP gain.

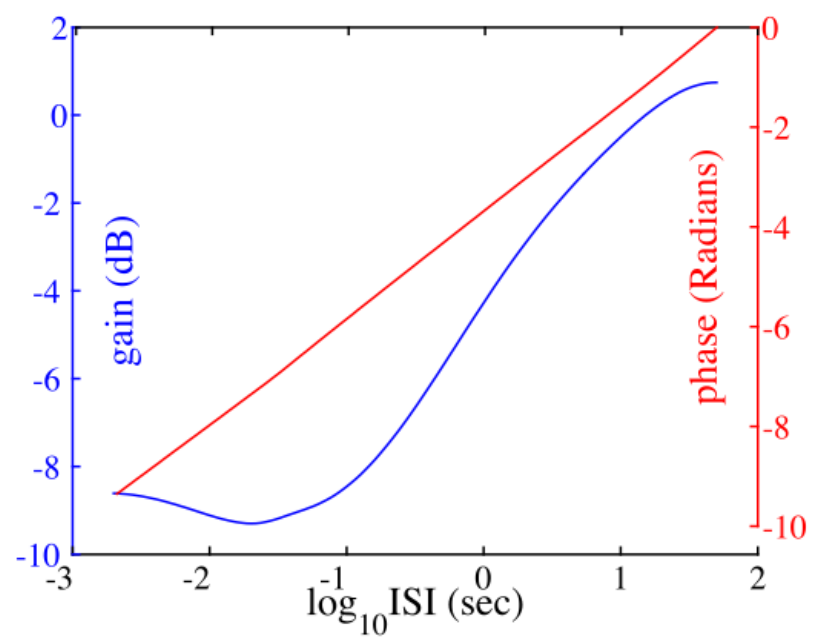

Figure 3.12. Gain and phase of filter corresponding to the group delay fit to DSCI STP gain. 


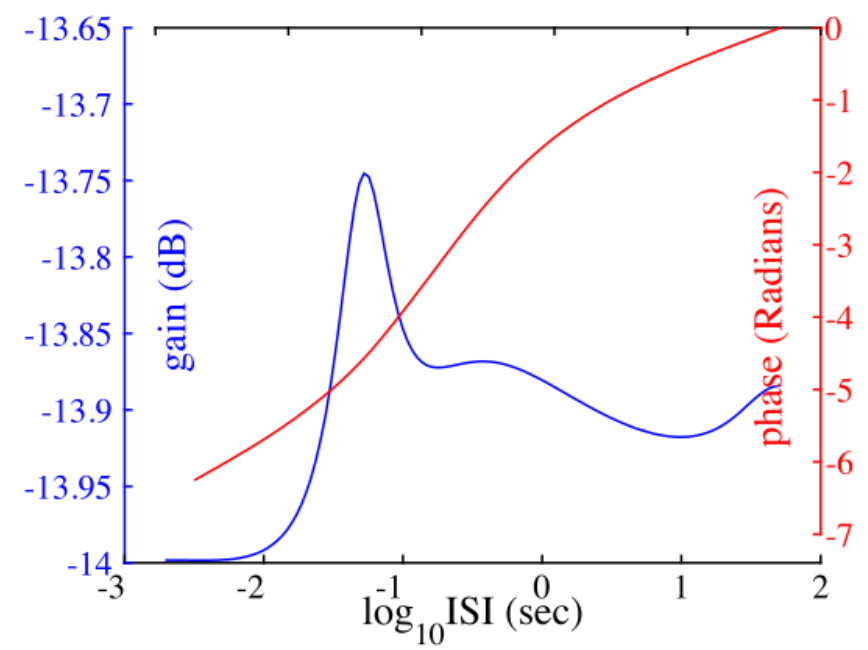

Figure 3.13. Gain and phase of filter corresponding to the gain fit to DSCI STP gain.

The gain vs. ISI ${ }^{-1}$ curves only take into account the immediately preceding ISI. However, STP gain is expected to show systematic variability as a function of more than one preceding ISI within the time window of use-dependency. For this reason, gain as a function of ISI doublets and triplets was also determined. The ISI triplet gain respnse forms a volume of data points, $y(\operatorname{ISI}(t-2), \operatorname{ISI}(t-1), \operatorname{ISI}(t-0))=f(\operatorname{ISI}(t-2), \operatorname{ISI}(t-1), \operatorname{ISI}(t-0))$. Slices through the volume can be obtained by constraining one of the dimensions to a subset of its domain. For instance, the first of the ISIs in the triplets can be held constant. Taking slices at constant values on the $\operatorname{ISI}(t-2)$ axis allows us to examine how the STP gain changes as the first ISI in a triplet changes. Six such interpolated surfaces are shown in Fig. 3.14. 
A

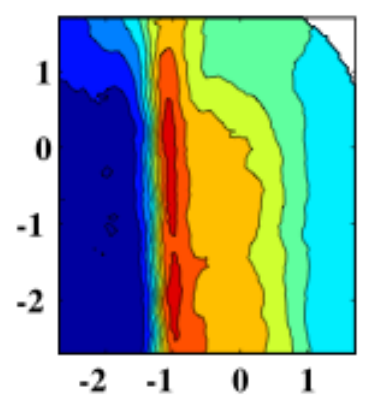

D

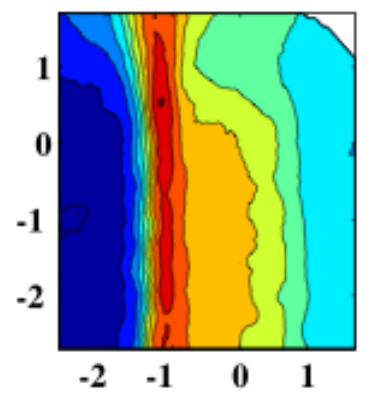

B

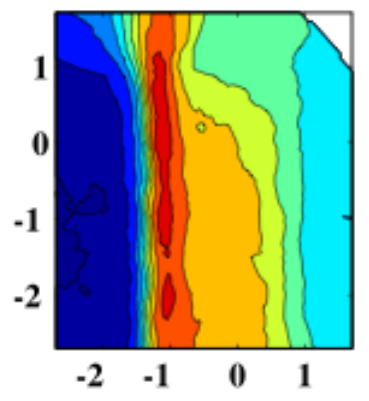

E

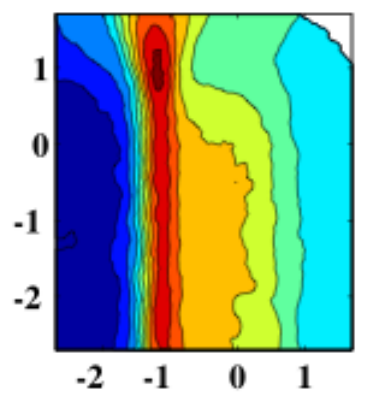

C
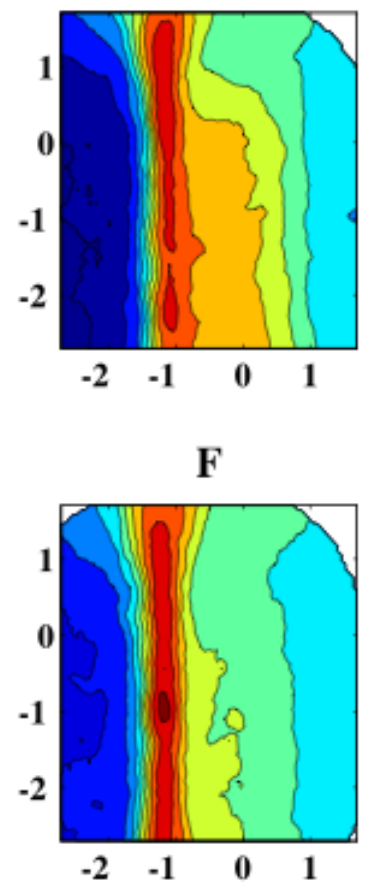

Figure 3.14. DSCI STP gain for different values of ISI $(t-2)$. A) $0.01 \mathrm{sec}, \mathrm{B}) 0.05 \mathrm{sec}$, C) $0.1 \mathrm{sec}$, D) $0.5 \mathrm{sec}$, E) $1 \mathrm{sec}$, F) $5 \mathrm{sec}$.

\subsubsection{Hypothesis 2:}

Changes in CAl model output train statistics vary non-linearly in response to linearly varying input train characteristics as a result of the inclusion of STP.

\section{Test}

Hypothesis 2 tests the idea that there are regimes in the characteristics of the inputs that are more sensitive to the effects of STP, and therefore more likely to generate outputs that are meaningful. Here "meaningful" implies the presence of a nonlinearity 
in some characteristic of the CA1 output as a function of a linear variation in some characteristic of the input. To test this hypothesis, two sets of ISI trains of similar duration (about 10 seconds) were drawn from the responsive empirical CA3 ISI distribu tion, and then interpolated in 10 discrete steps from a condition in which $80 \%$ of the 75 input trains were given no imposed correlation with complex train \#1 from the Frerking data, to the condition in which $80 \%$ of the 75 input trains were given $100 \%$ correlation with (i.e., made equal to) complex train \#1 from the Frerking data, resulting in 10 sets of inputs that varied smoothly between two extremes. These interpolated sets of input trains were applied to the CA1 pyramidal cell model with and without the STP models (models 1 and 2). Differences between the input and output trains under all three conditions (no STP, STP model 1, and STP model 2) were measured using the distance and rate metrics.

Four tests were performed at a mean sampling frequency of $1 \mathrm{~Hz}$, but with different rates of periodic modulation applied to the correlated trains. The four applied modulation rates were: $50 \mathrm{~Hz}, 75 \mathrm{~Hz}, 100 \mathrm{~Hz}$ and $125 \mathrm{~Hz}$. These rates were chosen to span the range over which gamma modulation would act. In fact, however, except for the $50 \mathrm{~Hz}$ modulation, these rates exceed the slow gamma modulation from CA3 acting upon the Schaffer collaterals. For this reason, this series of tests should be performed again with a modulation range that extends from about $20 \mathrm{~Hz}$ to about $50 \mathrm{~Hz}$. Stochastic synaptic background current was added according to [Destexhe et al, 2001] (see Appendix D). An additional separate set of four tests were performed under the same conditions, but 
also including modulation to the stochastic background noise at a rate of $25 \mathrm{~Hz}$. This rate is closer to the slow gamma from CA3. The higher frequency modulation might be interpreted as representing the fast gamma arriving on the distal dendrites from ECII, although during recall the distal inputs to CA1 may display less influence over CA1 behavior [Jones and McHough, 2011].

The mean van Rossum distance, $\langle\delta\rangle_{j}$, between the inputs trains and the output train at each interpolated step, $j$, was collected from the STP and non-STP tests. In addition, the mean rates, $\langle\theta\rangle_{j}$, of the input and output trains, for STP and non-STP tests, were recorded. For each of the four separate tests (at modulation rates $\phi_{i}$ ), 30 data points were collected, consisting of the pairs:

$$
\rho_{j}=\left\{\frac{\left\langle\theta_{\text {out }, \text { STP }}\right\rangle_{j}-\left\langle\theta_{\text {out } \left._{\text {nonSTP }}\right\rangle_{j}}\right.}{\overline{\left\langle\theta_{\text {in }}\right\rangle_{j}}},\left\langle\delta_{\text {STP }}\right\rangle_{j}-\left\langle\delta_{\text {nonSTP }_{j}}\right\rangle_{j}\right\} .
$$

The points, $\rho$, fell neatly on a straight line (not shown). The interpretation is that differences imposed upon spike trains by STP, as assessed by the van Rossum distance measure, could be explained by the differences in rate induced by STP effects. The slope and offset parameters of the linear fits were recorded for both models of STP (the parameters fit to the two data sets). Robust estimates of the error in the linear model parameters were obtained using nlinfit.m and nlparci.m in the Matlab statistics toolbox. 95\% confidence intervals for the linear model parameter estimations are shown in Figs. 3.15 and 3.16 for the two STP model tests described above. In all cases, the slopes are not significantly different between the two models of STP. However, the second model 
of STP does show offsets in the linear fits of the $\rho$ parameter that are significantly different than zero, unlike the first model of STP, implying that change in rate alone cannot explain the effect of STP in the second model.
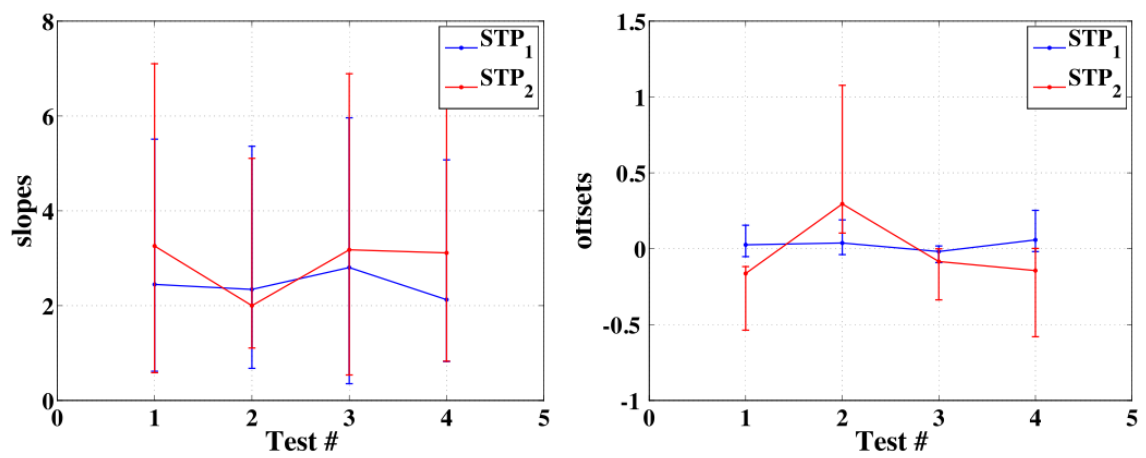

Figure 3.15. Slopes (left panel) and offsets (right panel) of linear fits of the test statistic, $\rho$, defined in Eqn.(3.6). Confidence intervals are 95\%. The morph condition in the four tests was the correlation of $80 \%$ of the 75 inputs with the complex train \#1 from the Frerking data (steps of 10 from $0 \%$ to $100 \%$ correlation). Tests 1 through 4 were performed with different rates of modulation applied to the correlation. In test 1 , the correlation was modulated at $50 \mathrm{~Hz}$, test 2 at $75 \mathrm{~Hz}$, test 3 at $100 \mathrm{~Hz}$, and test 4 at $125 \mathrm{~Hz}$. In all cases, background noise was included, but not modulated.
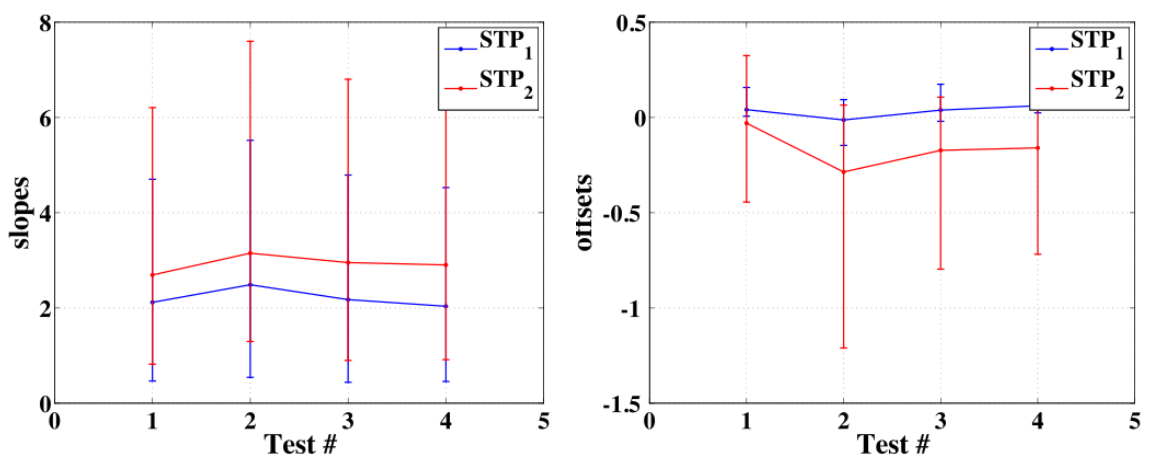

Figure 3.16. Same as Fig. 3.15, except with background noise modulated at $25 \mathrm{~Hz}$. 


\subsection{Summary}

Using the DSCI model, two distinct hypotheses about synaptic functionality were tested. The dynamic synaptic response to variable-ISI input trains drawn from a physiologically derived (empirical) ISI distribution shows a region of gain in the $10-40 \mathrm{~Hz}$ range. The physiological significance of this region of gain is explored more in chapter 4 , but in summary, this is the range of slow gamma oscillations generated in CA3 and used to convey information regarding the sequencing of events. The interpretation of the STP gain response has also been shown to potentially conform to filters operating in either the domain of group delay or of gain. Finally, STP was shown to have a linear variation in one measure of distance between input and output spike trains of a model CA1 pyramidal cell as a function of a linearly varying alteration in a characteristic of the input trains. This result could imply that, at least for the characteristic measured, STP does not preferentially increase response to characteristics of the input trains. This result does not rule out the possibility that there is alteration on shorter time scales than was observed, or to different characteristics than were tested. 


\section{Interpretation of STP the Schaffer collateral}

The goal of this chapter is to provide insight into the function of the Schaffer collateral in the context of hippocampal processing as it relates to cognition and cognitive pathologies. Behavioral, anatomical, and physiological observations of the hippocampal formation are compared with machine-learning concepts to provide a computational perspective on the operation of the hippocampus. With a broader perspective on the function of the hippocampus, our understanding of the role of STP at the Schaffer collateral may then be constrained.

The hippocampus is proposed to provide a solution to three dilemmas of storage and representation, including the balance of circuit stability with plasticity [Abraham and Robins, 2005], the maintenance of representational specificity and generalizability [Summerfield et al, 2011], and the translation between sparse and dense codes [Ahmed and Mehta, 2009; Willmore and Tolhurst, 2001]. STP at the Schaffer collateral is proposed to improve the sensitivity to certain regimes of operation by modulating the balance, timing, ratio, and/or influence of excitation and inhibition in the CA1 that is critical for the organization of associative and episodic memory [Hirase and Recce, 2011]. 


\subsection{Anatomy and function}

\subsubsection{A very brief history of hippocampology}

The modern era of hippocampal study began with the observation of patient "H.M.", who developed anterograde amnesia following surgery in which parts of his temporal lobes, including his hippocampi, were removed in order to relieve severe epileptic seizures [Scoville and Milner, 1957]. Anterograde amnesia is the inability to create new memories of events or episodes. Patient H.M. was able to remember items only for brief periods of time (some minutes) prior to distraction. H.M. also experienced retrograde amnesia extending back to approximately the period of time prior to the surgery during which he first experienced epilepsy. The case of patient H.M. motivated an era of animal studies in an attempt to understand the role of the hippocampus in amnesia and memory [Kandel, 2006].

Animal studies that took place in the 1960's failed to locate the hippocampus as the definitive site of memory consolidation. However, one finding that emerged from this period was the observation that hippocampectomized rats, while able to learn a conditioned behavior as well as normal rats, were less able to "withhold a prepotent behavioral response," that is, change behavior when conditions changed [Kimble, 1968; Anderson et al, 2007]. Hippocampectomized rats had no problem with simple Y- or Tmazes, but failed "miserably" in complex multichoice configurations (complicated mazes) [Kveim et al, 1964; Anderson et al, 2007]. 
A few things happened in the early 1970's that changed the experimental approach to hippocampal studies. First, long term memory was recognized as having different types. Endel Tulving classified memories according to their dependence on context [Tulving, 2002]. Memories that depended on context, he called episodic memories. Other memories, typically of the factual type, he termed semantic. Terry Winograd further categorized memory by grouping episodic and semantic memory types into declarative memory, in contrast to procedural memory, which refers to rehearsed skills and stimulus-response habits [Anderson et al, 2007]. The second thing to happen in the 1970 's was that behaviorists realized that their tests for animal memory were not optimal for observing hippocampal function [ibid]. As a result, more specific and appropriate object-recognition tasks were developed, allowing studies into the establishment of memory systems to gain more traction.

\subsubsection{Spatial representation}

Another major development in the 1970's was the use of implanted microelectrodes to monitor single-neuron activity in awake, behaving animals [Hirano et al, 1970; Vinogradova et al, 1970]. From the results of single electrode recordings, the cognitive map theory of the hippocampus was hypothesized [O'Keefe and Dostrovsky, 1971]. A cognitive map is a mental representation of the environment that provides navigational information. The cognitive map concept came out of the theory of expectancy, which explained experiments showing that rats could learn behaviors that were not directly reinforced (as strict behaviorism requires) [Tolman, 1948]. The theory of expectancy 
supercedes strict behaviorism by abstracting the stimulus-response paradigm to include a mental survey of surroundings that allows the flexibility to discover shortcuts or novel solutions to problems [Buckner, 2010].

According to cognitive map theory, the hippocampus supports spatial episodic memory formation and retrieval. Several electrophysiological findings supported this idea, including the discovery of place cells [O'Keefe and Dostrovsky, 1971]. Place cells are pyramidal neurons in the hippocampus that fire consistently when the test subject is in, or passes through, a localized region of an environment. The identification of spatially-sensitive receptive fields in the hippocampus resulted in a paradigm shift in the study of learning and memory [Mizumori, 2006].

Other cells that fire under specific conditions related to spatial awareness have since been discovered. Among these are head direction cells [Taube et al, 1990], border cells [Solstad et al, 2008], spatial view cells [Franzius et al, 2007], and perhaps most remarkably, grid cells [Hafting et al, 2005]. Grid cells have multiple regular firing fields that form two-dimensional triangular lattices covering an observable environment. The spacing of the lattices varies as a function of position on the dorsoventral axis of the EC, indicating that grid cells potentially convey metric information [de Almeida et al, 2009]. In lighted environments, grid cells orient to visual cues, but grid fields persist in the dark, so it is thought that the formation of grid fields is related to motion awareness (proprioception), as well as visual cues. Grid cells are not located in the hippocampus proper, but in the entorhinal cortex (EC). However, the superficial layers of the EC 
project directly to the $\mathrm{DG}$ and $\mathrm{CA}$, providing the hippocampus with its primary neocorti cal input.

The firing properties of spatially responsive cells change, or "remap", in different environments, or under varying behavioral circumstances [Colgin et al, 2008]. Remapping allows the hippocampal system to create context-specific representations of environments, which can be used to "disambiguate the behavioral contingencies" of similar stimuli that can occur in different spatial or behavioral contexts [Knierim et al, 2006]. In other words, different maps can exist for the same environment, but relating to different contingencies (i.e., contexts) [Leutgeb and Leutgeb, 2007].

\subsubsection{Navigation as cognitive template}

The prominent representation of space in the hippocampus and associated regions indicates the importance of the hippocampus for navigation [O'Keefe and Nadel, 1978]. At the same time, the hippocampus is crucially involved in the establishment, maintenance, and manipulation of episodic and declarative memories [Scoville and Milner, 1957; Buckmaster et al, 2004; Nadel and Hardt, 2010]. The interdependency of these functions may provide insight into the workings of the hippocampal region [Eichenbaum, 2004; Mizumori, 2004].

The convergence of spatial awareness with declarative memory formation in the hippocampal region might be understood as a solution to an organizational challenge. In the simplest terms, one might postulate that since the things we remember tend to be spatially located, the association of memories with positional awareness is the most 
parsimonious solution to the challenge of organizing memories. It may be harder, though, to explain how abstract declarative content that does not have a direct association to spatial awareness is nonetheless dependent upon the hippocampus. However, if we postulate that declarative memory formation employs the same organizational principles as used during the ongoing encoding of situational awareness, including route planning, then these principles might be understood as templates for organizing non-spatial associations as well.

A few examples might help make the point. For one, the phenomenon of transitive inference, or "the ability to integrate experiences that share overlapping elements and then use the consequent relations to guide novel judgments about elements that are indirectly related", has been found to involve the hippocampus [DeVito et al, 2009a; Reber et al, 2012]. Additionally, the ability to flexibly manipulate relationships between familiar items has been found to be dependent on the intact hippocampal system in primates [Buckmaster et al, 2004]. In rats, hippocampal region CA3, by supporting arbitrary associations, has been found to be required for relational encoding in episodic memory processing [Kesner et al, 2008].

Supposing then, that the navigational function of the hippocampus encompasses arbitrary associations, perhaps the scope of hippocampal function can be stated generally enough to characterize navigational processing in abstract cognitive spaces. To that end, it is proposed that the function of the hippocampus is to map attributes of objects and situations to locations on representational charts, that themselves are part 
of an atlas covering the space of known object-situation attributes. Memories of objectsituation interactions could either by located on existing charts, or on new charts, thereby forming a more complete atlas [Derdikman and Moser, 2010].

\subsubsection{Cognitive maps and schemas}

While cognitive map theory is strictly spatial, the survey of surroundings established in the hippocampus is thought to encompass "the full range of regularities present in the experience" [Eichenbaum et al, 1999]. If the hippocampus is indeed the locus of cognitive map generation, and if nonspatial information is combined with spatial information in the hippocampus, then spatial maps may represent only one aspect of the organization of cognition established in the hippocampus.

The generalization of the cognitive map is captured by the concept of the schema, which are mental models, or general cognitive structures that aid in making sense of, and remembering, new information. Schemas are "derived from an episode or prescribed activity, and are repeatable and generalizable to likewise future situations" [from http://www.nature-nurture.org/index.php/future-direction/appraisals/schemas-vsappraisals/], and represent domain-specific aspects of an individual's perceptual experiences and behavioral responses to those experiences [Piaget, 1972; Markus, 1977]. The distinction between the cognitive map and schema theories is simply that the cognitive map technically represents spatial awareness in isolation from other aspects of experience [Eichenbaum et al, 1999; Sweatt, 2004; Wood et al, 1999]. The schema theory seems to match Eichenbaum's broader interpretation of the function of place cells in 
the hippocampus.

It has been proposed and experimentally demonstrated that the hippocampus establishes schemas [Tse et al., 2007]. Tse demonstrated that hippocampally-established schemas allow for single-trial learning by first establishing a cognitive structure into which similar experiences can be rapidly added. In Tse's experiment, the schemas consisted of flavor-location paired associates (PAs). After extensive training over a period of weeks to familiarize six PAs, two new PAs were introduced, replacing two of the original six PAs, but were introduced in only one training session. The recognition of the new PAs that were introduced after the familiarization period with a fixed set of PAs was significantly greater after 24 hours, than was the recognition of the new PAs following a training period in which the training PAs were not fixed, but instead varied every day. An NMDA receptor-dependent mechanism was later identified as specifically responsible for encoding the new PAs [Bethus et al, 2010].

In a direct connection with computational intelligence theory, schemas parallel the contextual manifold concept of Lendaris and Santiago [Lendaris, 2009; Santiago, 2004]. The contextual manifold instantiates a continuous framework for the organization of existing knowledge into which new knowledge can be readily interwoven and indexed. For example, in reference to control theory, a context, as defined by Lendaris, consists of a triplet of a plant, an environment, and a criterion function, where a plant is a controllable system, an environment is a set of parameters that affect the behavior of the plant, and a criterion function is the definition of performance for the plant in the 
environment. The contextual triplet indexes into a repository, or manifold, of controllers that have been learned through prior experience [Lendaris, 2009].

The major advantage of contextual organization, especially in high-dimensional systems, is that items are located based upon some measure of similarity [Kanerva, 1993]. Such an organizational strategy simplifies the task of storing new information, and making inferences based on the likelihood that information located in nearby coordinates has more relevance than information stored in distant coordinates. Essentially, a contextualized organizational strategy performs a process known as manifold learning, whereby high-dimensional data is mapped to a lower dimensional space (an abstracted level of representation), while preserving metrical and/or topological relation. ships in the data, thereby forming a coherent basis for "reasoning about the world" [Roweis and Saul, 2000], or making informed decisions.

\subsubsection{Anatomical subregions}

At this point, the discussion turns to a review of the anatomy, physiology, and function of the hippocampus in preparation for elaborating on the proposed organizational strategy of the hippocampus and the similarity of that strategy to the contextual manifold principle.

The hippocampus is characterized by a stark division of anatomical subregions, each of which performs a unique function, influenced by the upstream (afferent) biasing from region(s) receiving some set of input features in common with the biased efferent region(s). The subregions of the hippocampus and their major connections with the 
neocortex are illustrated in Fig. 4.1.

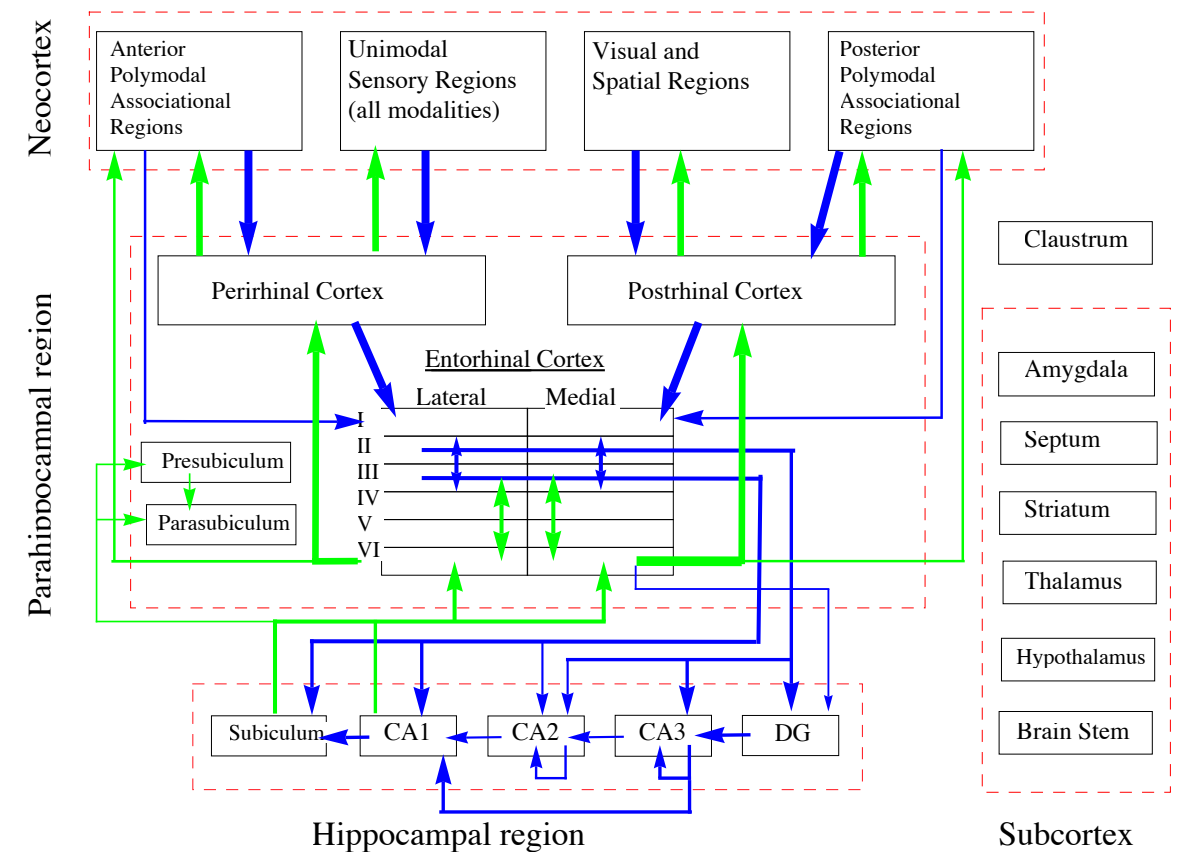

Figure 4.1. Basic cortico-hippocampal connections. Descending pathways in blue; ascending in green.

Both neocortical and subcortical projections converge on the hippocampus. The neocortical projections convey sensory representations relating to spatial and object awareness. Some areas of the neocortex connected to the hippocampus, including the orbitofrontal [Brown et al, 2010] and medial prefrontal cortex [DeVito and Eichenbaum, 2011], are implicated in sequential event anticipation, novelty detection, and flexible response selection and suppression. Subcortical projections provide global modulation related to attention, reward, timing and emotional relevance. Subcortical regions also display functions related to the organization of responses (amygdala, 
nucleus accumbens, basal ganglia, hypothalamus), and "internal adjustments related to the anticipation of action" (amygdala, hypothalamus) [Cotterill, 2001; McNaughton, 2006].

The combination of neocortical and subcortical projections suggests that enough information potentially converges on the hippocampus to represent the sensory, motivational and emotional aspects of experience. Behavioral observations tend to agree with the anatomical suggestion. The hippocampus has an observed function in the formation and retrieval of spatial [O'Keefe and Nadel, 1978; Moser et al, 2008], contextual [Nadel et al, 1985; Levy et al, 1995; Treves, 2009; McHugh and Tonegawa, 2009] and relational [Buckmaster et al, 2004; Kesner et al, 2008] aspects of episodic memories. The hippocampus is tightly connected with the amygdala, providing direct access to emotional content of memories [Phelps, 2004]. The hippocampus is also active during goal-oriented behavior and spatial navigation [O'Keefe and Nadel, 1978; McNaughton, 2006; Vinogradova, 2001]. Furthermore, hippocampal memory traces can be flexibly applied in contexts different than in which they were learned [Acsady and Kali, 2007], and learning has been observed to accelerate after contextual information is consolidated [Tse et al, 2007].

\section{Entorhinal cortex}

Almost all of the neocortical projections to the hippocampus funnel through the entorhinal cortex (EC) [Amaral and Lavenex, 2007]. The neocortical projections to the EC are typically categorized into two pathways originating in sensory and associational 
regions of the neocortex, and terminating in the lateral and medial entorhinal cortices (LEC and MEC, respectively) [Canto et al, 2008]. The two pathways process spatial and object information mostly independently, displaying a pattern of convergence to the EC, followed by largely reciprocating divergence back to the neocortex [Lavenex and Amaral, 2000], implying that inputs and outputs are kept mostly in register, thereby potentially preserving topological relationships between representations in the EC and the rest of the neocortex [Agster and Burwell, 2009]. The two pathways are associatively mixed in the hippocampus, and probably in the entorhinal cortex as well [Eichenbaum and Lipton, 2008; Canto et al, 2008].

Layers I-III of the entorhinal cortex (the superficial layers), receive most neocortical projection, while layers IV-VI (the deep layers), receive most hippocampal projections. The superficial EC layers II and III project to the hippocampus, while the deep EC layer $\mathrm{V}$ projects back to the neocortex. One of the exceptions to this rule is that layer $\mathrm{V}$ also sends some projections to the DG [Canto et al, 2008; Amaral and Lavenex, 2007], possibly implicating layer $\mathrm{V}$ in the comparison (match/mismatch) function variously ascribed to hippocampal and extra-hippocampal regions [Kumaran and Maguire, 2007; Villarreal et al, 2007; Hasselmo, 2005; Vinogradova, 2001].

Layers II and III interact strongly, displaying a pattern of divergenge from II to III, and convergence from III to II. Layer II has two types of excitatory cells: pyramidal and stellate. The pyramidal cells receive most inputs from other layers in EC, while the stellate cells send outputs to the hippocampus and broadly innervate EC layer I, possi- 
bly indicating surround inhibition, since layer I contains mostly interneurons. The apical dendrites of layer III and V pyramidal cells are narrowly constrained and terminate in layer I, indicating specificity of cortical inputs (bearing in mind the influence from layer II). Basal dendrites of EC V are clustered near the soma, also indicating input specificity from the hippocampus. Deep layer inputs to superficial layers are known to integrate position, direction and speed information [Sargolini et al, 2006], providing more specificity.

Anatomical connections in EC indicate that the deep and superficial layers interact [Beed at al, 2010; Canto et al, 2008]. Deep to superficial projections are predominant, mainly from EC V to EC III, and have been found to be spatially clustered, implying segregation of computational units. The report of clustered projections must be weighed against another report, finding that pyramidal cell axons in layers II and V both extend broadly in their respective layers (with layer $\mathrm{V}$ also innervating layer III in a convergent manner), while layer III axons tend to be more confined to a cylindrical region around the soma, and extend through all layers [Quilichini et al, 2010]. The spatial clustering of deep layer projections reported by Beed potentially indicates that deep layers perform a more selective operation, while the broader dendritic and axonal influence in the superficial layers suggests more of an associative function. However, according to Quilichini et al, EC III may be responsible for the limited spatial extent of excitability between deep and superficial layers, implicating layer III in the coupling of EC input and output representations. 
Inter-layer interaction in the $\mathrm{EC}$ has been observed in the form of "reverberant activity" lasting for more than 200ms after a single stimulus in the superficial layers [Iijima et al, 1996]. In this study, stimulation of EC layer II generated activity in the deep layers of EC before activity was observed to spread to the hippocampus. The activity in the EC was subsequently sustained in part by hippocampal outputs from CA1 and subiculum. This type of study has several caveats: the stimulation pattern is completely unlike anything that would be seen in vivo, and the resolution of the optical signal is low. Also, as in most ex-vivo hippocampal studies, the slice preparation severs the temporo-sagital connections, possibly removing important components of the circuit. Nevertheless, this study does suggest that an observable interaction occurs along the anatomical connections between the EC and hippocampus.

Inputs to the hippocampus from EC layer III, but not from layer II, have been found to be crucial for temporal association memory, and have been shown to maintain persistent activity up to several minutes, during the delay period of working memory tasks, possibly accounting for the temporal association signal [Tahvildari et al, 2007; Suh et al, 2011]. The persistence of firing of EC III cells was found to be an inherent property of the neurons [Hasselmo et al, 2010]. Layer V cells were found to share this property. Layer III cells were also found to be more excitable and have a higher mean firing rate than cells in other layers [Quilichini et al, 2010].

Grid field expression in the EC is correlated with place cell activity in the hippocampus, and is suggested to form a spatial basis for contextual representation [Gustafson 
and Daw, 2011]. Layer II stellate cells provide the main input to the DG and CA3, and express only grid fields [McNaughton et al, 2006]. The offset, spacing, and orientation of grid fields in EC layer II is independent of the size or shape of the environment, and all grid offsets (phases) are equally represented within a small region of EC [Hafting et $\mathrm{al}, 2005]$. The spacing and orientation of neighbouring grid cells is almost identical, but their grids are offset relative to each other in an apparently random manner. Cells in layers III and V express grid fields, head direction cells and the conjunction of grid fields with head direction cells, which are also sensitive to speed.

Global remapping of place fields in the hippocampus corresponds to grid reallignment in the MEC, while rate remapping in the place fields does not correspond to rate remapping in the grid fields [Fyhn et al, 2007]. Ensembles of grid cells remain coactive during global remapping in hippocampal area CA3, and the alignment of grid fields is unaltered during rate remapping in CA3. To place the characteristics of grid cells in the context of the proposed function of the hippocampus, it would appear that the active ensemble of grid cells represents the spatial basis for the current conceptual chart. Rate remapping in the hippocampus would then correspond to transformations on that chart, while global remapping represents a transition between charts, and possibly the formation of new charts.

\section{Dentate gyrus}

Many pieces of behavioral evidence correlate the dentate gyrus (DG) with learning. Some of those behavioral results are briefly summarized here before the computational 
role of the DG is discussed. The dentate gyrus seems to be most active during the early training period of tasks requiring spatial and temporal memory, as compared to CA1 and CA3 [Poirier et al, 2008], and there is evidence that spatial representations in the DG form immediately in new environments [Hill, 1978; Wilson and McNaughton, 1993; de Almeida, et al, 2009]. The DG appears to be required when similarity between place-object association is maximal, but not when place-object association similarities are low [Lee and Solivan, 2010]. Similarly, lesions of the DG impair the detection of metrical, but not topological alterations to locations of objects [Kesner, 2007]. Transgenic mice with deleted NR1 subunit of the NMDA receptor show impaired memory performance in a partial-cue, but not a full-cue environment [Bischofberger et al, 2006, citing Nakazawa et al, 2002]. Finally, loss of inhibition in aging DG/CA regions, leading to over-excitability, has been tied to "behavioral discrimination deficits", specifically, a reduced sensitivity to differences between similar objects [Yassa and Stark, 2011].

The progressive convergence of pathways from the neocortex to the hippocampus suggests that the compression from neocortex to hippocampus allows regionally dense, but globally sparse coding to flourish in the neocortex [Kaiser and Varier, 2011; Manns and Eichenbaum, 2006; McClelland and Goddard, 1996]. Dense codes have the advantage of being generalizable and fault tolerant (due to redundancy), but the output is complex (i.e., not linearly separable) [Foldiak, 2002]. The hippocampus, on the other hand, expresses an exceptionally sparse (almost local) encoding scheme, as exempli- 
fied by place cells [Ahmed and Mehta, 2009]. During navigational tasks, only a small percentage of the activity in the EC generates activity in the hippocampus [Coulter and Carlson, 2007]. Specifically, only about $2 \%$ of the principal granule cells in the DG are active during navigation [Chawla et al, 2005]. The sparsity of coding in the hippocampus indicates that the hippocampus translates the dense representation in the EC into a sparse code that facilitates the rapid formation of "multidimensional memory items" [Ascady and Kali, 2007]. The dentate gyrus is uniquely adapted to perform this sensitive translation [ibid].

Despite the sparseness of DG activity, there are about four times as many DG principal granule cells as ECII stellate cells [Wilson et al, 2006]. The divergence of the EC projections to the DG implies dimensional expansion, a technique often used in statistical data analysis to preprocess data for non-linear classification [Vapnik, 1999]. Inhibitory interneurons in the DG are thought to operate competitively with the granule cells to remove redundancy from the inputs, producing the sparse, orthogonalized, representation, as characterized by the emergence of place cells [Kesner, 2007]. The competitive mechanism in the DG has been shown to potentially operate through a selforganizing process [Rolls et al, 2006], and the DG has also been compared to the support vector machine algorithm [Baker, 2003]. The DG is one of only two regions in the brain known to generate new cells well into adulthood [Kesner, 2007; Aimone et al, 2011; Zhao et al, 2008; Deng et al, 2010], which might suggest a flexible learning process akin to the growing self-organizing map (GSOM) [Alahakoon et al, 2000], or 
the growing cell structure (GCS) [Fritzke, 1994].

Pattern separation is preserved at the input to the CA3 due to the low degree of connectivity between the DG and CA3 (about $0.005 \%$ ), carried on the so-called mossy fiber (MF) axons. The low degree of connectivity is balanced by the strength of the mossy fiber synapses. Although a large majority $(\approx 95 \%)$ of MF synapses terminate on inhibitory interneurons in the CA3, the minority of MF synapses that terminate on excitatory cells have multiple active zones and sodium channel densities more character istic of axons, rendering the synapses exceptionally reliable [Bischofberger et al, 2006]. Mossy fibers synapses also terminate proximally onto the apical dendrites of CA3 pyramidal cells, providing unusually strong excitatory coupling [Acsady and Kali, 2007]. Furthermore, MF synapses establish a narrow and precisely timed window within which perforant path (PP) inputs from ECII can contribute to the depolarization of the CA3 pyramidal cells [Urban et al, 2001; Bischofberger et al, 2006]. The temporal precision of the MF bouton has been compared to that of the calyx of Held, which is known to play a key role in temporal processing in the auditory brainstem [Bischofberger et al, 2006].

The temporal windowing function of the DG signal has been explained by the difference in the synaptic transmission between mossy-fiber boutons and filopodial synapses that branch off of the mossy fiber boutons and synapse onto DG inhibitory interneurons. The MF synapses exhibit a very large facilitation (up to $600 \%$ going from $0.05 \mathrm{~Hz}$ to the $1 \mathrm{~Hz}$ range), while the filopodial synapses show a much smaller facilitation 
(about 200\%). Granule cell activity switches the DG-CA3 influence from inhibitory to excitatory as a function of firing frequency [Bischofberger et al, 2006; Coulter et al, 2011]. High frequency granule cell bursting shifts the balance between monosynaptic excitation (DG granule cell to CA3 pyramidal cell) and disynaptic inhibition (DG granule cell to DG interneuron to CA3 pyramidal cell) towards excitation. Through this dynamic competition between excitation and inhibition, only the granule cells with the strongest activity convey excitation to the CA3. DG granule cells are also gated by their own intrinsic inexcitability, via lack of regenerative calcium conductances and a very hyperpolarized resting state [Coulter et al, 2011].

Gating of EC activity by the DG seems to perform a protective function for the highly excitable and plastic CA3 region. However, the gating might have more than a protective function. The temporal precision and nonlinearity of the mossy-fibre (MF) windowing of the CA3 pyramidal cells are indicative of a "teacher" function [Bischofberger et al, 2006; de Almeida et al, 2009]. Dendritic switching by means of a gating process could instantiate a context-switching process [Jeffery, 2011], and might suggest a relation to the information bottleneck theory of learning, as proposed in [Buesing and Maass, 2010]. Is it possible that coincidental activity of the MF and the PP could establish the conditions for a manifold-learning process in the CA3 by enforcing a topologically consistent dimensional reduction of the EC inputs?

To summarize this brief review of the DG, it appears that the spatio-temporal sensitivity of the DG facilitates the translation of densely coded signals in the EC to the 
sparse code observed in the hippocampus. The sparse output of the DG, projecting directly and solely to the CA3, disproportionately influences a small subset of CA3 principal cells. How then does the CA3 respond upon receiving the DG signal?

\section{CA3}

The Cornu Ammonis (CA) region is divided into three subregions: CA1, CA2 and CA3. The subregions are distinguished by the characteristics and composition of their neuronal populations, as well as the targets of their axons and sources of input to their dendrites [Amaral and Lavenex, 2007]. All CA subregions receive input from the entorhinal cortex, via EC layers II or III, but not all CA subregions receive the same complement of EC inputs [ibid]. CA3 receives cortical inputs mainly from ECII, while CA1 receives mainly ECIII projections. However, the majority of synapses onto CA3 pyramidal dendrites originate from CA3 pyramidal axons, in the form of recurrent connections. A degree of recurrent connectivity of $2-4 \%$ implies that any given CA3 pyramidal cell can influence any other CA3 pyramidal cell within as few as three recurrences [Rolls and Treves, 1998].

Epileptic seizures in the medial temporal lobe appear to originate in the CA3, due to the massive recurrence [Coulter et al, 2011]. The CA region is also particularly sensitive to ischemia, and it has been shown that CA1 cells can be spared from transient forebrain ischemia if the connection from CA3 is severed [Onodera et al, 1986]. However, the traits that make the CA3 region prone to seizure and ischemia are probably also important for the information processing role that the hippocampus plays. For 
example, the bursting behavior of CA3 cells [Xu and Clancy, 2008; Chevaleyre and Siegelbaum, 2010] may facilitate information transfer, even though potentially risking the induction of seizure due to the excitatory positive feedback.

The distinctive firing properties of $\mathrm{CA} 3$ cell populations support the fast acquisition (one-trial learning) of detailed memories, by maintaining a "locally continuous, globally orthogonal" representation that facilitates the rapid integration of novel sensory inputs into a previously learned framework [Rolls, 2007; Leutgeb and Leutgeb, 2007]. The CA3 has been found to begin encoding place field sequences immediately upon encountering novel configurations of familiar environmental landmarks [Kneirim et al, 2006]. This property may help explain behavioral findings showing that memory formation is more rapid if an existing and related mental schema or experience already exists [Tse et al, 2007; Wang and Morris, 2010; Bahar et al, 2011; van Kesteren et al, 2012].

In cooperation with the $\mathrm{CA} 1$, the $\mathrm{CA} 3$ has been shown to play a role in the sequential processing (temporal pattern separation/completion) of information [Jerman, et al 2006; Vago et al, 2007]. The CA3 and CA1 were both found to be required for the recall of multiple places in a sequential maze-running trial, but pharmaceutical lesions of the perforant path projection to CA1 impaired between-day learning (i.e., retrieval) [Vago et al, 2007], while CA3 lesions impaired within-day learning (encoding) [Jerman, et al 2006]. The CA3 has also been found to support arbitrary paired-associate learning [Kesner et al, 2008]. The idea is that the CA3 holds an item in memory 
until another item comes along, at which time the second item is associated with the first by means of "temporally asymmetric synaptic associativity" (i.e., spike-timing dependent plasticity) [Rolls and Kesner, 2006].

The excitatory recurrence in the $\mathrm{CA} 3$ has prompted comparison to recurrent neural networks of two main types. The first comparison is to the recurrent autoassociative network, originally suggested by David Marr [Marr, 1971], and epitomized by the Hopfield network [Hopfield, 1982]. The second CA3 comparison is to the "attractormap" network, inspired by the asymptotic behavior of recurrent neural networks [Colgin et al, 2010; Jeffery, 2011]. In both comparisons, the recurrent network displays attractor dynamics. However, in the autoassociative network, the modifiable synapses of the recurrent collaterals establish stable attractors representing input features, while in the attractor-map network, modification of the input synapses alters the dynamics of pre-existing attractors. The attractor-map network need not have stable attractors, and may even benefit from a degree of instability [Legenstein and Maass, 2007].

The anatomy of the CA3 supports the idea that it functions as an autoassociative network [Rolls and Treves, 1998]. Behavioral evidence has also emerged supporting the $\mathrm{CA} 3$ as an autoassociative network: NMDA receptors in area CA3 are required for place field stability and in order to remember the location of a goal when presented with an incomplete set of cues, implying that plasticity in CA3 synapses is required to retrieve a complete map from a partial set of cues [Nakazawa et al, 2002]. Input from the DG is thought to trigger an autoassociative recurrence in the CA3 that reconstructs 
a meaningful sequence of events that (temporally) contextualizes the state of the ECII, and applies that generalization to the CA1 [Tsukada and Fukushima, 2010]. CA3 cells have been found to more closely follow one set of cues than do CA1 cells, indicating more constraint to a single map and tighter binding to upstream coordinate systems [Eichenbaum et al. 1999]. Studies of aging animals also indicate a potential pathology in this system, since older animals show a tendency to use previously learned, but unrelated maps when switching between environments [Leutgeb and Leutgeb, 2007].

Data from individually recorded CA3 neurons indicates that the network enters different, and possibly unique, tonic states modulated by input stimuli [Vinogradova, 2001; Wiebe and Staubli, 1999]. In the Wiebe and Staubli study, rats were habituated to a "complex" task, but still had to make a decision involving both spatial and olfactory information in a delayed match-to-sample task. Some CA3 cells were found that discriminated between one or more of the parameters of the task, including odor, location, and performance. The recorded ISIs displayed a range of mostly non-Poisson distributions, including many that were distinctly multi-modal. Precisely correlated activity between cells was reported as modest, but loosely correlated behavior was found to be common [Frerking et al, 2005]. Non-random interactions between cells, lasting as long as 10 seconds, accounted for about $10 \%$ of the spike patterns. Interestingly, the spike patterns that could not be explained by random sampling were more likely to have been produced by cells that responded to environmental stimuli [ibid]. 
On the other hand, evidence from cultured hippocampal slices indicates a minimal influence of stimulation on the modulation of CA3 network states [Sasaki et al, 2007]. Metastable states in the CA3 were observed that showed resistance to perturbation by inputs from the DG, unless the inputs were "sufficiently strong" [ibid]. The CA3 dynam ics were stable for tens of seconds, transitioned abruptly to new states, and rarely revisited previous states. These data suggest that the recurrent CA3 network inherently supports attractor dynamics, and that the influence of the inputs is less predicitive of the network dynamics than is the current state of the network. A caveat with this result is that it was obtained from cultured tissue, so the state of the network may not bear exact resemblance to the in vivo network. Cultured slices do, however, retain more recurrent connections in the CA3 circuit than do acute slices [ibid].

Inherent metastability seems to be a characteristic of in vivo CA3 dynamics as well, though. For instance, CA3 place field activity tends to remain fairly consistent while an aspect of environmental context is slowly varied, until the variation more closely resembles another context, at which point the activity pattern shifts in an abrupt but coordinated remapping of place fields [Wills et al, 2005; Bischofberger et al, 2006]. This behavior was observed by training rats to recognize square and circular rooms, and then observing CA3 activity while the shape of the square room was gradually altered in discrete steps to resemble the circular room. An earlier review also concluded that the CA3 responds nonlinearly to variations in contextual similarity [Guzowski et al, 2004]. 
An exhaustive review of place cell behavior in response to morphed enclosures concluded that external inputs are secondarily associated with groups of neurons active at a given location [Colgin et al, 2010]. Experiments performed by Colgin et al were consistent with this conclusion (and also with [Leutgeb et al, 2005]), indicating that, “... although external features and events may affect the relative firing rates of the set of hippocampal neurons belonging to a particular coordinate representation, they typically scarcely affect the membership of the set. In contrast, changes in spatial location produce changes in the active set if the differences are sufficiently large" [Colgin et al, 2010]. These findings suggested to the authors that the autoassociative interpretation of the CA3 was unlikely, and that the region more probably served to create "phase sequences" of place codes that could "encode the coordinates of a route as a vector field amenable to flexible route selection" [ibid].

\section{CA1}

CA1 place cells have been found to be more responsive to similarities between tasks, while the opposite (greater responsiveness to differences) was seen in CA3 place cells [Bahar et al, 2011]. In comparison to CA3 cell ensembles, which were found to be distinct for separate rooms, regardless of the similarity between rooms, the overlap between CA1 cell ensembles increased as a function of similarity of room [Leutgeb et al, 2004]. CA1 cells also show a lesser degree of coherence with "disconcordant cue manipulations" than do CA3 cells, a behavior that may result from the lack of recurrent connections [Leutgeb and Leutgeb, 2007]. Diversity of response may be one of the 
defining characteristic of CA1 neuronal activity.

Place cells in the CA1 are also responsive to sensory features that are not uniquely related to spatial processing [Leutgeb and Leutgeb, 2007]. Such conjunctive place cells in CA1 are natural candidates for the identification of points, and their trajectories, on the object/space charts that encode episodic memories [Komorowski et al, 2009]. However, the translation to ECV is meaningful only if it corresponds to the appropriate chart. The identity of the chart must then be determined by the pattern of activity in the superficial layers of the EC, as transformed by the $\mathrm{CA} 2$ or DG/CA3. It must be mentioned, though, that the EC projections to CA2 pass through and synapse on the subiculum. EC layers II and III both innervate the CA2, which is able to activate the CA1, which in turn projects to the subiculum. Outputs from the CA1 and the subiculum converge on ECV. EC layer III might set up a rough expectation of object placement in the subiculum, which gets refined by $\mathrm{CA} 2 / \mathrm{CA} 1$, transformed again in the subiculum, and finally combined with direct output from CA1, in ECV. The topography of the projections to and from the $\mathrm{EC}$, that run through the $\mathrm{CA} 1$ and subiculum, is preserved and reciprocated [Tamamaki and Nojyo, 1995]. It is possible then, that the subiculum supports the selection of the appropriate chart in ECV. This explanation would have the benefit of including the influence of subcortical modulation directly in the chart selection process.

Coincidental input timing is also implicated in the activity of the CA1 region, since the temporally-specific arrival of inputs along the Schaffer collateral (SC) gates the 
inputs from the temporoammonic (TA) pathway from the EC at a specific phase relationship with respect to the theta-frequency network oscillation [Coulter et al, 2011]. Since the SC inputs are more proximal on the CA1 pyramidal dendrites, and thus more depolarizing than the TA inputs, which are limited to the apical tuft and rarely depolarize the soma on their own, the activity in the CA3 establishes the conditions by which the CA1 is sensitive to inputs from the EC.

The CA1 and subiculum also send projections directly to the medial prefrontal cortex (mPFC), and receive indirect excitatory projections from the mPFC by way of the nucleus reunions (in the thalamus) [Vertes, 2007]. The indirect inputs from the mPFC are proposed to activate the CA1 independently of either the CA3 or the EC [Bahar et al, 2011]. This pathway of activition was invoked to explain results indicating CA1 activity in the "absence" of CA3 place cell activity during a novel task [ibid]. Although there were fewer CA3 cells expressing place fields (about half as many as during the habituated tasks), there were more that did not display place fields, and although these other cells have to be assumed to have been silent during the trials, the data didn't indicate that the CA3 was completely devoid of activity as was suggested in the conclusions. Nevertheless, the CA1 is known to operate in the absence of CA3 input. The gating properties of the CA1 dendrites make it unlikely that the TA pathway is responsible for CA1 activity in the absence of CA3 inputs. However, until recently, the $\mathrm{CA} 2$ region has rarely been brought into the discussion, and since the CA2 region can excite the CA1, and it receives inputs from ECII and III, it remains a possible 
driver of CA1 activity not mentioned in [Bahar et al, 2011].

\section{CA2}

The CA2 field receives strong, direct inputs from both ECII and ECIII, implicating the CA2 region in coordinating activity between the trisynaptic and monosynaptic hippocampal pathways [Jones and McHough, 2011]. There is evidence that the CA2 forms a disynatpic pathway with CA1, but does not form a quadrisynaptic pathway with CA3 and CA1 [Chevaleyre and Siegelbaum, 2010]. As in the CA3 and CA1, the perforant path (PP) projects onto the distal dendrites of CA2 pyramidal neurons. However, unlike regions CA3 and CA1, which can only be indirectly activated by PP inputs, CA2 can be activated directly by PP inputs [Bartesaghi and Gessi, 2004]. CA3 Schaffer collateral (SC) projections pass through and synapse onto CA2, but have an inhibitory influence. CA2 pyramidal cells do not express spines on the proximal apical dendrite (unlike the CA1 and CA3 pyramidal cells) - possibly explaining the weaker influence of the proximal inputs [Amaral and Lavanex, 2007]. In addition, CA2 dendrites have fewer branches in stratum radiatum, compared with CA1 dendrites [Chevaleyre and Siegelbaum, 2010]. Region CA2 sends axons to both basal and apical CA1 dendrites, and excites region CA1 via strong unitary connections. CA2 pyramidal cells are also reported to display excitatory recurrent connectivity [Amaral and Lavanex, 2007], but this feature isn't often mentioned in the more recent literature.

The CA2 displays different LTP properties than the adjacent areas CA1 and CA3 [Frerking, personal communication]. CA3-to-CA2 connections fail to undergo LTP 
using typical protocols [Simons et al, 2009]. However, the distal synapses upon which ECII and ECIII inputs converge undergo robust LTP (which is pathway specific, i.e., ECII or ECIII inputs can independently or cooperatively induce LTP) [Chevaleyre and Siegelbaum, 2010].

The emerging picture is that region CA2 is involved in modulating the timing of the inputs from ECII and ECIII to the hippocampus, thereby coordinating the conjunction of different aspects of episodic experience [Jones and McHugh, 2011]. Mice that lack the vasopressin $\mathrm{V} 1 \mathrm{~b}$ receptor, which is highly expressed in CA2, show selective impairment in temporal event memory [DeVito et al, 2009b]. Lesions along the CA3/CA2 border also impair hippocampal operant conditioning (i.e., learning complex relationships between multiple stimuli that have varying degrees of association with a given response) [Samuel et al, 1997]. Along with the DG, region CA2 is the only hippocampal structure targeted by the supramammillary nucleus, a structure involved in controlling the frequency of theta activity [Chevaleyre and Siegelbaum, 2010] (see discussion of oscillatory activity in section 4.1.6).

Alterations in the balance of excitation and inhibition in region CA2 are known to correlate with some cognitive pathologies. For example, a reduction in interneuron expression in region $\mathrm{CA} 2$, leading to increased excitability in the region, has been correlated with schizophrenia and manic depression [Benes et al, 1998]. In addition, an overexcited CA2 has been linked to the spread of epileptic acitivity in the hippocampus [Williamson and Spencer, 1994]. However, region CA2 has been reported to show less 
vulnerability to neurotoxicity caused by sustained activation of excitatory amino acid receptors than the $\mathrm{CA} 1$ or $\mathrm{CA} 3$, making the $\mathrm{CA} 2$ region resistant to epileptic damage [Bartesaghi and Gessi, 2004]. Likewise, region CA2 is more resitant to damage induced by Alzheimers, hypoxia and head trauma.

\section{Subiculum}

The subiculum combines laminar and columnar microcircuits, forming a transition zone between neocortex (EC) and archicortex (hippocampus), where the lamination provides dense interconnectivity between the columns. The subiculum is also highly connected to a wide range of subcortical areas, suggesting that the subiculum is responsible for conveying modulatory infomation to and from the hippocampus [O'Mara, 2006]. These connections place the subiculum in a position to monitor the hippocampal inputs and outputs and relay the results back to the EC. Multiple place cells from CA1 converge upon single subiculum cells [Amaral and Lavenex, 2007], and CA1 and entorhinal projections synapse topographically and in-register in the molecular layer of the subiculum (onto pyramidal cell dendrites). CA1 projections synapse deeply in the molecular layer (proximal on the pyramidal dendrites), and entorhinal projections synapse superficially.

Whereas hippocampal place fields specialize to environmental details, subicular place fields generalize details, can expand and contract to different sized environments, and often display directional information in conjunction with place-related patterns [Sharp, 2006; O’Mara, 2006]. To quote O'Mara, “... subicular units tend to fire through- 
out the environment and show multiple peaks of activity; in general, subicular place fields appear to be of lower resolution and comprise much larger areas of comparable environments than those of area CA1" [O'Mara, 2006]. The finding that subicular representations can expand or shrink while retaining their topographical specificity may indicate a more topological than metrical role for the subiculum.

The broad and general receptive fields of place cells in the subiculum might encode enough information about the environment to enable novelty detection and rudimentary navigation. The subiculum may play a role in translating the cortical representation, which resembles general basis functions, to the hippocampal representation, which displays specificity. Perhaps the representation in the subiculum is akin to a set of basis functions that represent topological aspects of environments, such as borders, corners, intersections and the like.

\section{Subcortical regions}

The hippocampus is under subcortical modulatory influence, and also influences several subcortical systems. Subcortical inputs to the hippocampus include the septal nucleus and diagonal band, the contralateral hippocampus, as well as the amygdala, brainstem, hypothalamus and thalamus [Brown and Zador, 1990]. The septal inputs enter through the fimbria, dorsal fornix, supracallosal striae and the amygdaloid complex. The contralateral hippocampal inputs enter through the fornix. Brainstem inputs include a noradrenergic input from the locus coeruleus, a serotonergic input from the raphe nuclei and a dopaminergic input from the ventral tegmental area. There is a 
hypothalamic input from the mamillary bodies through the fimbria and a ventral route to the DG, CA2 and CA3. Also the anterior thalamic nucleus projects to the subiculum.

There are two major outputs of the hippocampus, one from the subiculum, and the other from the hippocampus proper [Martin, 2012]. The outputs from the subiculum collect in the fornix and project to mammillary bodies of the hypothalamus, completing a processing loop, part of the Papez circuit, which is involved in the cortical control of emotion. Outputs from the CA1 also project directly to the septal nuclei and to the entorhinal cortex, both directly, and indirectly through the subiculum.

\subsubsection{Coordinated activity}

Activity in the hippocampus and elsewhere in the brain is observed to be coordinated by the synchronous firing of neurons expressed by the local field potential (LFP) (although most spiking in the hippocampus happens in the absence of coordinated oscillatory activity [Frerking, personal communication]). In the hippocampus, LFP behavior is categorized into three main frequency ranges: theta, gamma, and sharp waves [O'Keefe, 2007]. The categories of oscillatory LFP activity are defined by their passbands. Theta occurs between about 4-12Hz, gamma between about $20-140 \mathrm{~Hz}$, and sharp waves around $140-200 \mathrm{~Hz}$.

Theta band activity is observed in all hippocampal and associated regions, but is most pronounced in area CA1 [Ang et al, 2005]. Hippocampal theta activity is correlated with neocortical and subcortical theta activity, so is proposed to be the rate at which experience is discretized and synchronized with other brain regions [Colgin and 
Moser, 2010; Hyman et al, 2011], and may represent a timing signal to which sequences of events are bound [Lisman et al, 2005; Mizuseki et al, 2009]. Theta-entrain. ment of mPFC cells has been found to be predictive of the outcome of working memory task trials, accounting for the firing rate of the mPFC cells, implying that more information is carried on the theta code during working memory tasks than can be accounted for by a simple rate code [Hyman et al, 2011].

The behavioral actions of exploration and learning co-occur with theta activity. Specifically, the hippocampal operations of encoding and retrieval are suspected to be correlated with the phase of the theta cycle [Hasselmo et al, 2002; Kunec et al, 2005; Villarreal et al, 2007]. In favor of this hypothesis, the preferred phase of firing, with respect to the theta LFP, shows a decrease in CA1 (but not in subiculum) that is correlated with the degree of sensory novelty, indicating that novel and familiar signals are separated as a function of theta phase [Lever et al, 2010]. Additionally, it has been found that states in the CA3 can switch, i.e., completely remap, within a theta cycle [Jezek et al, 2011].

Persistently firing EC layer III cells have been found to have "pronounced frequency specificity", which, across the population of cells, spans the theta range [Erchova et al, 2004]. However, layer III frequency specificity is limited to about $10 \mathrm{~Hz}$, or around the upper limit of theta, and the cells are inhibited by higher frequency inputs. Layer II cells are sensitive to inputs greater than $5 \mathrm{~Hz}$, or about the lower limit of theta. Thus, there is an overlapping region of sensitivity in EC layers II and III, 
approximately in the theta band, but layer II also responds to higher frequency stimulation.

Gamma band activity is superimposed upon the theta cycle, and has been correlated with episodes that are later recalled [Sederberg, et al, 2003; Montgomery and Buzsaki, 2007]. Theta and gamma synchrony between prefrontal cortex and hippocampus are both observed, possibly during different behavioral conditions [Benchenane et al, 2011]. Phase-amplitude cross-frequency coupling between theta and gamma oscillations (the modulation of gamma by theta waves) has been associated with the formation of item-context associations [Tort et al, 2009], and variations in the character of theta-modulated gamma between encoding and retrieval are reported (see Figs. 4.2 4.4) [Jones and McHugh, 2011]. Gamma-frequency inhibition in the CA3 has been suggested to prevent persistent attractor states, for which, as of 2007, there was apparently no experimental evidence [de Almeida et al, 2007; Levy et al, 2005] (although see [Lisman, 1999, citing Vinogradova, 1984; Hampson et al, 1993; Colombo and Gross, 1994]).

Hippocampal gamma oscillations are further observed to cluster into two distinct regimes: slow gamma (about 20-40Hz) and fast gamma (about $60-140 \mathrm{~Hz}$ ), that are generated independently in the CA3 and EC, respectively [Colgin and Moser, 2010]. Fast and slow gamma waves are generated intrinsically in the subiculum, where independent gamma phase modulation of cells is thought to allow selective participation in neural synchrony [Jackson et al, 2011]. 


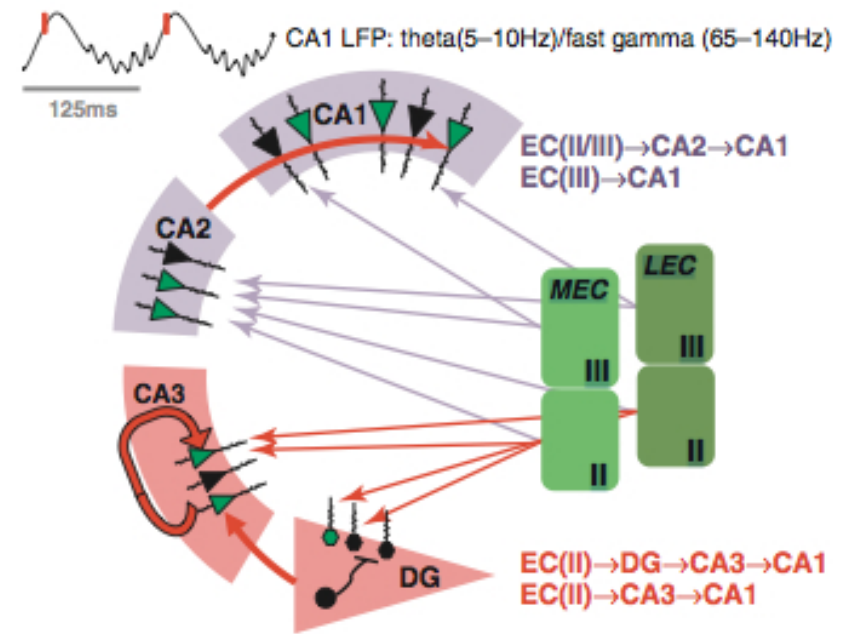

Figure 4.2. Jones and McHugh's proposed hippocampal encoding model. Figure credit: [Jones and McHugh, 2011]

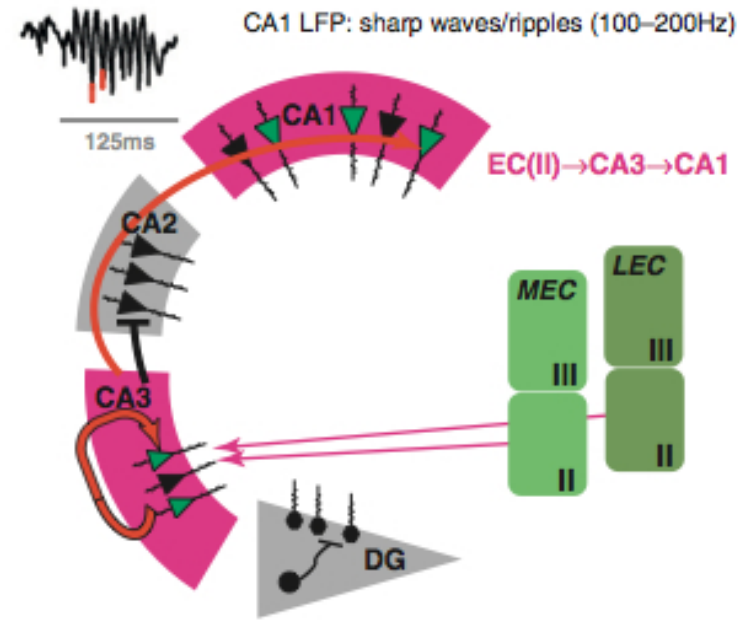

Figure 4.3. Jones and McHugh's proposed hippocampal consolidation model. Figure credit: [Jones and McHugh, 2011] 


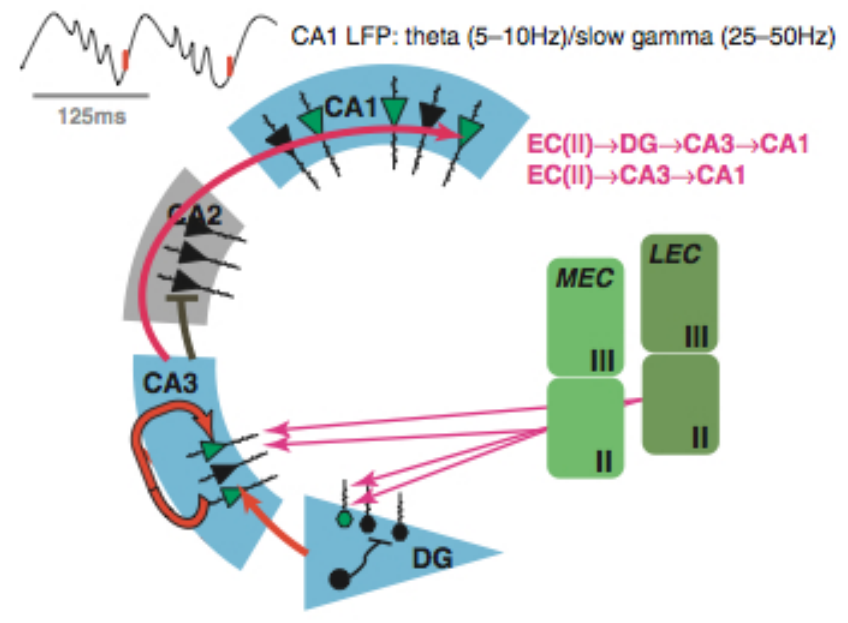

Figure 4.4. Jones and McHugh's proposed hippocampal recall model. Figure credit: [Jones and McHugh, 2011]

Place fields are represented at the gamma frequency, and as an animal moves through space, place cell activity precesses across the theta wave [O'Neill et al, 2010]. In the CA1, place field activity has been estimated to occur at frequencies greater than $50 \mathrm{~Hz}$ in about half of the spikes [Harris et al, 2001; Harvey et al, 2009]. Theta phase precession has also been interpreted as a cued prediction of the sequence of upcoming positions [Lisman and Redish, 2009]. Theta phase precession has been observed in the DG, CA, and superficial EC [Yamaguchi et al, 2007]. Although grid cells in EC layer III don't display theta phase precession, grid cells in EC III preferentially fire on only one half of the theta wave [Quilichini et al, 2010; Jones and McHugh, 2011].

Sharp wave ripples are brief bursts of coordinated principal cell activity that originate in the CA3 [Csicsvari et al, 2000]. Sharp wave ripple (SPW-R) activity occurs during slow-wave sleep and periods of inactivity, and has been associated with increased hippocampal-cortical communication [Csicsvari et al, 2000; Davidson et al, 
2009]. The level of spiking synchrony is about tenfold more during SPW-R than during theta activity [Gasparini and Magee, 2006]. During SPW-R activity, subcortical neuromodulator activity is reduced, causing disinhibition in the spread of activity in the CA3 collateral system [Hasselmo et al, 1995], and EC layers II and III are "relatively silent" [Chrobak and Buzsaki, 1994]. SPW-Rs are believed to be an intrinsic HPC network pattern that is present when the cortical and subcortical influences are reduced, since lesions of the EC increase the incidence of sharp waves and fast gamma frequency, while decreasing the amplitude of theta waves [Bragin et al, 1995].

SPW-Rs are regarded as signals that are appropriate for studying the CA3-to-CA1 connectivity [Csicsvari et al, 2000]. The cells observed firing during sharp wave activity are the same cells observed previously firing in theta during the encoding of episodes, with a preserved forward or reverse order of firing [Foster and Wilson, 2006; Gupta et al, 2010]. Therefore, it is possible that SPW-Rs represent the replay of attractors in the CA3 unconstrained by theta and gamma modulation.

SPW-R activity is thought to be associated with the consolidation of memories [Eschenko et al, 2008]. Historically, the protocol for LTP-induction in the hippocampus has consisted of a one second application of a high frequency (e.g., 100Hz) signal [Lawrence et al, 2009]. This protocol is not physiologically representative, and does not work well with frequencies less than $50 \mathrm{~Hz}$ [Behrens, et al, 2005]. However, LTP can be readily induced in the in-vitro and in-vivo hippocampus using a "theta" protocol consisting of $30-40 \mathrm{msec} 100 \mathrm{~Hz}$ bursts repeated for 5 seconds at a rate of $5 \mathrm{~Hz}$ [Staubli 
and Lynch, 1987; Nguyen and Kandel, 1997]. The in-vitro range of CA1 sensitivity to LTP using the theta-protocol spans the whole theta range, is maximal around $2 \mathrm{~Hz}$, and extends down to $0.05 \mathrm{~Hz}$, thereby including rates at which sharp wave bursts repeat during slow wave sleep [Grover et al, 2009].

\subsubsection{Novelty detection}

Novelty detection is a fundamental property of adaptive behavior [Kumaran and Maguire, 2007]. The detection of novelty has been variously attributed to region CA1 [Karlsson and Frank, 2008], CA3 [Villarreal et al, 2007; Vinogradova, 2001], the entorhinal cortex [Lorincz and Buzsaki, 2001], all the hippocampal regions (particularly the subiculum) [McNaughton, 2006], subcortical regions (reticular system via the medial septum [Vinogradova, 2001]), and the parahippocampal cortex [Howard et al, 2011]. It is safe to say that there may be more than one system involved in novelty detection. Likewise, novelty also has multiple facets. The hippocampus seems responsive to associative and contextual novelty [Kumaran and Maguire, 2007]. Associative novelty is defined by the occurance of familiar items in new spatial or temporal arrangements. Contextual novelty consists of an event or stimulus that is unexpected given the context. These two novelty types are constrasted by stimulus novelty, which is defined as an item never experienced before [ibid].

In general, novelty detection involves the comparison between an expected and an observed signal, thus requiring access to the input and output signals of a modeling region. If the observed and expected signals are the input and output from the DG and 
CA regions, then this constraint limits the location to somewhere between EC layers II and $\mathrm{V}$, potentially including the subiculum. At least one computational model suggests that novelty is detected by comparing the activity between EC layer II and layer V, the latter of which is influenced by hippocampal processing [Lorincz and Buzsaki, 2001].

Deep layer EC neurons have been found to fire more rarely than the superficial layer EC neurons during initial exposure to a novel environment, becoming more active during periods of rest [Burgalossi et al, 2011]. The deep layers also seem to be selectively activated by sharp wave activity, which is suspected to be correlated with memory consolidation [Chrobak and Buzsaki, 1994; Chrobak et al, 2000]. These results would indicate at least two things: first, that EC deep layer learning is indeed hippocampus-dependent, and second, that EC deep layers are involved in novelty-awareness, potentially through an inverse dependence on activity. If the deep layers of the EC maintain the current set of navigational charts (the atlas), then a lack of activity might indicate a mismatch between the patterns of inputs to the superficial layers, and the expected tranformation from the $\mathrm{CA} 1$ and subiculum.

Further evidence that the EC plays a role in novelty detection comes from lesion studies. Hippocampectomized animals display "insatiable curiosity", by showing defective habituation to reaction to novelty, indicating the importance of hippocampal outputs for the identification of novelty [Vinogradova, 2001]. Removing the cholinergic inputs to the EC selectively impairs non-match-to-sample performance for novel but not familiar odors [McGaughy et al, 2005]. Also, direct electrical stimulation 
applied to the EC, but not the hippocampus, has been found to improve spatial memory performance in humans when the stimulation is applied during the learning phase, presumably when the novelty signal is largest [Suthana et al, 2012].

Bax gene knockout mice, in which DG cells do not develop normally, show an impaired ability to locate targets when dead-reckoning and visual information are incongruent [Lee (CCJ) et al, 2009]. In this study, the EC-CA3 connection was apparently unimpaired. The implication of this study is that the DG is required for the associa tion of internal maps with external object location. The study also suggests that the hippocampus establishes a training signal for the cortical processing between layers II and $\mathrm{V}$ of the EC. If so, the hippocampus would have to be able to establish a faithful model of the world at the level of abstraction in the EC, which would align with the Lorincz-Buzsaki model.

Other computational models of the hippocampus [Hasselmo and McLelland, 1999; Rolls, 1996, 1989] suggest that the CA3 is involved in encoding and retrieval, while the CA1 is involved in match/mismatch detection operating on the temporoammonic and Schaffer collateral inputs. In these models, a mismatch between inputs excites the medial septum, raising acetycholine (ACh) levels in the CA3, attenuating the recurrent collaterals, and establishing an encoding phase. A match between inputs does not activate the medial septum, consequently allowing recurrent activity to dominate in the CA3, setting up retrieval. According to Hasselmo's model, scopolamine (a cholinergic antagonist) should, by inhibiting ACh, selectively impair encoding, whereas physostig- 
mine (an acetylcholinesterase inhibitor) should, by inhibiting the breakdown of ACh, selectively impair retrieval. [Rogers and Kesner, 2004] tested Hasselmo's cholinergic modulation model of encoding and retrieval using a task that was sensitive to hippocam. pal disruption. Scopolamine was found to disrupt encoding but not retrieval. Physostigmine was found to disrupt retrieval but not encoding. The results suggested that increased ACh levels are necessary for encoding new spatial contexts, and decreased ACh levels are necessary for retrieving previously learned spatial contexts.

The EC has also been shown to generate oscillations thought to be important in setting up theta modulation in the hippocampus [Dickson et al, 2000]. The EC could establish theta from the septum to the hippocampus, increasing ACh and activating the DG-CA3 pathway, thus facilitating encoding. During retrieval, the EC could activate the PP to the CA3 directly, resulting in little ACh increase. According to [Rogers and Kesner, 2004], Egorov's 2002 paper [Egorov et al, 2002] shows that the EC can sustain activity over a time delay, also implicating EC layer $\mathrm{V}$ as the potential match/mismatch mechanism. This picture aligns with the [Rolls, 1996] model suggesting that the DG mossy fiber inputs onto CA3 are responsible for encoding, and that direct PP inputs are responsible for retrieval.

Rogers and Kesner's 2004 results support both the Hasselmo and the Buzsaki models, but cannot distinguish between them. However, when combined with data from [Lee and Kesner, 2004] and [Rogers and Kesner, 2003], the findings support the Lorincz-Buzsaki model. [Lee (I) et al, 2005] found that CA1 lesions had at most a modest 
effect on novelty detection of recently familiarized (within three minutes) objects in new locations, which would also argue against Hasselmo's model since the CA1 is therein responsible for the match/mismatch operation. [Lee (I), et al, 2005] found instead that the DG and CA3 significantly influenced novelty detection beyond the level observed in the CA1 lesioned animals. Hasselmo responded to the [Lee (I) et al, 2005] results by considering the role of retrieval played by CA3 in novelty detection [Hasselmo, 2005]. Since the test for novelty detection used by Lee (I) et al required retrieval from a set of partial cues, it would be expected that the CA3 would be required for detection of novelty [ibid].

Hasselmo's response addressed the role of the DG and CA3. However, the fact that lesioning the CA1 had a lesser effect on novelty detection is puzzling, since the CA1 provides hippocampal output to the cortex, specifically EC, layer V. One explanation is the CA1 lesions in the study were incomplete [Hasselmo, 2005]. Another is that the subiculum also takes part in novelty detection [McNaughton, 2006]. It is also possible that there is a novelty detection process that occurs subcortically [ $\mathrm{Li}$ et al, 2003], perhaps in conjunction with the parahippocampal region [Furtak et al, 2007], and is involved with the hippocampus through the CA3, since the CA3 also has subcortical connections. The CA1 may also be more involved in detecting novelty under conditions in which recollection of "older" memories is involved [Lee (I) et al, 2005]. Finally, the Lee (I) et al study, like most studies, could not isolate the CA2, which was affected by lesions of both the CA3 and CA1 [ibid]. 


\subsubsection{Contextual processing}

The contextually motivated acquisition and consolidation of information may be one of the hallmarks of hippocampal operation [Smith and Mizumori, 2006; Vann and Albasser, 2011]. The explicit idea of contextual sensitivity in the hippocampus seems to have originated in 1974 [Hirsch, 1974], and has been defended as a means of coping with "predictable ambiguity" [Morris, 2007]. Morris described experimental evidence for the contextual theory of hippocampal function, although he conceded that many of the experiments were vague or even confusing when it came to the definition of context. In setting the stage for his discussion of the experimental paradigms of contextual theory, Morris was careful to state that some aspects of contextual encoding, modulation and recall are hippocampus-dependent, while others are not.

The original experimental paradigms supporting the contextual theory involve fear conditioning, in particular, "context-freezing". In these experiments, a context (a box with a conductive grid floor), is associated with a mild electrical shock, the response to which is sustained immobility, or "freezing" of the animal test subject. After this response is learned, the animal is removed from the context for a period of time, and then returned to the context. If the hippocampus of the animal has been immediately lesioned, little of the conditioned response is observed. Furthermore, the freezing response increases with the time between conditioning and lesioning. In all cases, the fear response can be extinguished with repeated exposure to the context in the absence of the fear stimulus, over a period of weeks. [Rudy et al, 2002] also found that an intact hippocampus was necessary for the association of independent features (i.e., the con- 
text) with an aversive stimulus, and [Kim and Fanselow, 1992] found that the hippocam pus mediates "contextual conditioning", which is conditioning paired to the context of the stimulus, so that the response is present in the absence of the stimulus, but the presence of the context.

A thread from a slightly older hippocampal theory, known as the declarative theory, concerns a potential model of long term consolidation [Morris, 2007]. In this theory, the hippocampus indexes (or points to) locales in the cortex and "cautiously" interleaves new information into existing encodings. Could this process be facilitated, both in terms of efficiency and continuity, by a topological association of indices formed in the hippocampus? This suggestion is not too different from what has been proposed in the spatial domain [Samsonovich and McNaughton, 1997]. Samsonovich showed, through simulations, that an animal's position in space could be mapped as quasicontinuous fixed point attractors on multiple 2-D manifolds. Nor is the suggestion of topological association of indices vastly different from Hasselmo's alternative explanation for the role of the DG/CA3 as "encoding the relationship between objects dependent on the relative times or locations at which they were encountered, providing a mechanism for relational memory" [Hasselmo, 2005].

Perhaps topological properties, such as nearness and homotopy, transfer to the neocortex as well, carrying with them the topological associations of space and context in episodic memory traces at the highest level of abstraction. In an extension of the fear conditioning experiments, it was found that if there are three contexts, $\mathrm{X}, \mathrm{Y}$, and $\mathrm{Z}$, of 
which, $\mathrm{X}$ and $\mathrm{Y}$ contain common elements, and $\mathrm{Z}$ is quite different, that the conditioned fear response will be observed in $\mathrm{X}$ and $\mathrm{Y}$, but not $\mathrm{Z}$, if the response was conditioned in context Y [Rudy and O'Reilly, 1999]. This result may indicate the presence of a mental construct that confers an analog to topological nearness upon contextual fear conditioning.

\section{The context discerning multifunction network}

To be useful, an abstracted indexing system must maintain some measure of similarity to the thing it indexes. Related items should maintain a metrical similarity between the space of the explicit and the space of the abstract (which, in the brain, would not necessarily imply topographical organization). Contextualizing abstracted or hidden variables with metrical, or at least topological consistency, has been proposed as a way to efficiently optimize control policies in rapidly changing processes [Lendaris, 2009], and as a way of solving reinforcement-learning problems in non-stationary environments [da Silva et al, 2006].

Research in artificial neural networks has yielded an architecture that can discover the meta-level correlational rules that govern the output of a non-stationary dynamical system [Lapedes and Farber, 1986; Prokhorov et al, 2002; Santiago, 2004]. The network architecture, dubbed the "context discerning multifunction network" (CDMN) by Santiago, has a master/slave organization, where the master and slave networks receive the same inputs, but the master network abstracts the input to a sparse representation that biases the operation of the slave network towards a specific region of operation. 
This arrangement of networks extends the capability of the neural network from approximating functions to approximating functionals (a functional is technically a map from a vector space into its underlying scalar field, or intuitively, a function of a function i.e., the topological space is a function space) [Back and Chen, 2002; Santiago, 2004]. In essence, the master network learns the functionality of the slave. In the time-dependent CDMN, the master circuit is instantiated by a recurrent neural network (RNN) that learns a family of fixed point attractors from the training set, and subsequently biases a subset of the nodes in the slave circuit. In the CDMN, the slave circuit is a feedforward muti-layer perceptron (MLP).

The hippocampus appears to share some architectural similarities to the CDMN. In particular, there appears to be a separation of biasing, or context-level, and output, or action-level, networks that receive similar inputs [Hirsch, 1974]. The ECII $\rightarrow$ DG/CA3 circuit has been suggested to operate specifically as a temporal contextual processing circuit [Hasselmo and Eichenbaum, 2005]. Hasselmo and Eichenbaum's proposed functional diagram of the hippocampus is shown in Fig. 4.5, and can be compared to the CDMN architecture, as shown in Fig. 4.6. In the ECII $\rightarrow$ DG/CA3 circuit, it appears that the DG either biases or triggers the CA3 [Doboli et al, 2000], perhaps in the same way that the RNN biases the MLP in the CDMN. 


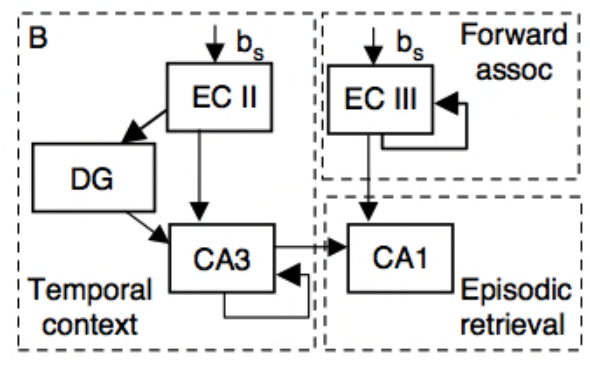

Figure 4.5. Hasselmo and Eichenbaum's proposed functional organization of the hippocampus and EC [Hasselmo and Eichenbaum, 2005]. The circuit in panel B bears architectural and functional similarity with the context discerning multifunction network shown in Fig. 4.6 [Santiago, 2004]. Figure credit: [Hasselmo and Eichenbaum, 2005]

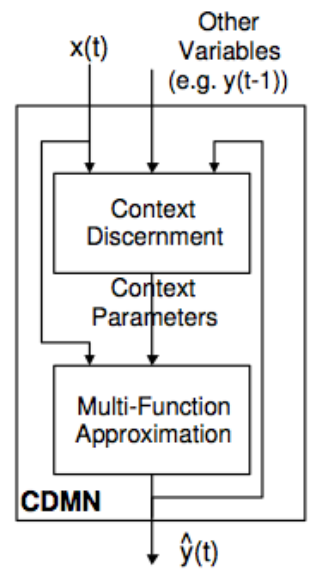

Figure 4.6. Architecture of the context discerning multifunction network [Santiago, 2004]. Note that the multi-function approximation stage sends a signal back to the context discernment network. Axons have been found that project from CA3 to DG [Scharfman, 2007]. Figure credit: [Santiago, 2004]

The pattern of context/action network differentiation also seems to appear in the $\mathrm{ECII} / \mathrm{ECIII} \rightarrow \mathrm{CA} 3 / \mathrm{CA} 1$ and $\mathrm{ECII} / \mathrm{ECIII} \rightarrow \mathrm{CA} 2 / \mathrm{CA} 1$ circuits. Even though the inputs from ECII and ECIII are not identical, the information is correlated since the layers are adjacent and heavily interconnected. Regardless, the context-action relationship also appears to exist, with the outputs from either the CA3 or CA2 biasing the CA1 neu- 
rons, thereby affecting the depolarizing potential of ECIII inputs.

The novelty of the CDMN paradigm is that the network learns to dynamically recognize distinct but related families of input-output mappings with fixed weights, after the training period [Santiago, 2004]. The biasing from the master network mirrors the topological consistency in the family of input-output functions, and allows interpolation to functions not explicitly included during training. The CDMN has been shown to be effective in simple low-dimensional problems [ibid]. However, the operational requirements of the hippocampus are high-dimensional and more involved. For one thing, the training and recall phases of operation are ongoing in the hippocampus, and appear to be interleaved at the theta rate [Kunec et al, 2005]. For another, the temporal relationship between events in a sequence of occurrences (including actions, and potentially decision points) is encoded in the hippocampus, whereas in the CDMN it is not. Also, the hippocampus is able to learn completely new families of input-ouput functions, presumably without losing the previous mappings, and is able to learn from a single presentation. The question that remains in order to draw a parallel between these two architectures is whether the hippocampal subregions and their interactions, as well as interaction with other limbic and cortical regions, allow for the operational elaborations beyond the CDMN without utilizing a fundamentally different network strategy.

\subsection{What does this have to do with STP at the Schaffer collateral?}

To summarize the main conclusion from the previous sections in this chapter: the hippocampal system organizes experiences into a relational framework that utilizes and 
maintains topological characteristics of the cortical representation of experience. The hippocampus is required in order to integrate the ongoing representation of experience into a continuous stream that can be mentally revisited [Lorincz and Szirtes, 2009; Wikenheiser and Redish, 2012]. If the organization of experience according to contextual relationships requires a means of recognition, then the hippocampal formation must be able to reconstruct arbitrary aspects of prior experiences from an arbitrary set of stimuli [Szirtes et al, 2004; Duncan et al, 2012; Meyer, 2012]. Additionally, the hippocampus seems necessary to imagine alternative outcomes of experience [Buckner, 2010; Gupta et al, 2010].

System identification is the process that circumscribes the construction and reconstruction functions proposed for the hippocampus [Lorincz and Szirtes, 2009; Berger et al, 2010; Buckner, 2010]. System identification involves the development of a model that can simulate arbitrary aspects of a dynamic process in order to predict the results of variations made to the inputs of the process [Ljung, 1987]. In the case of the hippocampus, the modeled process corresponds to the ongoing sequence of abstracted multi-modal stimuli that represent generic observations of cause and effect in the allocentric reference frame [Lisman and Otmakova, 2001; Treves, 2004; Lorincz and Szirtes, 2009; Lisman and Redish, 2009; Muezzinoglu et al, 2010]. The predictive performance of hippocampal system identification has implications for cognition [Berger et al, 2010; Addis et al, 2010; Meyer, 2012] and mental health [Green et al, 2005; Siekmeier et al, 2007; Behrendt, 2010; Bast, 2011]. 
One of the challenges that the hippocampal system must deal with in order to accurately model experience, is filtering the dense inputs from the entorhinal cortex [Coulter et al, 2011]. The hippocampus is proposed here to divide this filtering challenge in two computational stages that can be identified with connectionist paradigms. First, the dentate gyrus (DG) transforms the dense cortical code into a sparse representation through a dimensional expansion analogous to a "self-organizing map" (SOM) [Kohonen, 1993] (more specifically, a "growing" SOM, or GSOM [Mole and Araujo, 2010], or potentially a similar construct called a "growing cell structure" (GCS) [Fritzke, 1994]). The function of the DG is often identified with "pattern separation" [Myers and Scharfman, 2010; Yassa and Stark, 2011], although it has been suggested to correspond better with "memory resolution" when neurogenesis is taken into account [Aimone et al, 2011]. Next, the sparse representation in the DG triggers an associative signature embodied by a dynamic trajectory through the recurrent network of the CA3 [Behrendt, 2010]. The function of the CA3 is often identified with "pattern completion" [Myers and Scharfman, 2010].

Although the activity of the CA3 has been strongly implicated in associative processing [Kesner et al, 2008; Nakashiba et al, 2008; Ramamoorthi et al, 2011], as of yet, no perfect analogy to a computational paradigm for the CA3 exists [Colgin et al, 2010]. The evidence does not, it would seem, exclude a type of recurrent network known as a "liquid state machine" (LSM) [Maass, 2010]. The LSM network is composed of randomly connected excitatory and inhibitory computational units (spiking neurons), and 
is championed for its ability to simulate arbitrary dynamics without necessarily adjusting synaptic weights [ibid]. Instead, for an LSM, learning consists of discovering the appropriate weighted combination of its output neurons (usually a linear combination thereof) [ibid]. The conditions that have been proposed to be required for an LSM to operate, namely, a sparse set of inputs and an output network that can interpret the dynamic state of the LSM [ibid], are present in the hippocampus: the DG provides the sparse inputs, and the CA1 interprets the outputs of the CA3.

The hippocampus is proposed here to translate neural spike codes between the hippocampal and cortical representations in the EC, resulting in an enhanced associative flexibility of the compressed cortical signal. This translation could represent the brain's solution to the "stability-plasticity dilemma", that is, the trade-off in representational specificity inherent in balancing the ability to form new associations with the ability to recall previously encoded associations [Abraham and Robins, 2005; Grossberg, 2009]. According to the present proposal of the hippocampal function, the medial temporal lobe has solved the stability-plasticity dilemma by dividing the stable and flexible representational systems into separate regions (the EC and the hippocampus, respectively) [Manns and Eichenbaum, 2006].

The enhanced plasticity of the hippocampus renders it sensitive to over-excitation. However, gating mechanisms limit the amount of activity reaching the most plastic regions of the hippocampus (the CA3 and the CA1) [Ang et al, 2005; Ang et al, 2006; Coulter et al, 2011; Jones and McHough, 2011]. Hippocampal gating mechanisms 
filter the persistent EC activity in two ways [Ang et al, 2005; Ito and Schuman, 2012]. The first mechanism, corresponding to the action of the dentate gyrus, is a winner-takeall discriminatory operation that directly compresses the dense cortical code from the EC into a sparse representation in the higher-dimensional space of the DG [de Almeida et al, 2009]. The second gating mechanism indirectly affects the influence of the cortical inputs to the distal dendrites of the pyramidal cells in the CA regions by varying the phase relationship of frequency-modulated inputs that converge from different regions [Urban et al, 2001; Buzsaki and Draguhn, 2004; Ang et al, 2005; Coulter et al, 2011].

In light of the functional and anatomical organization of the gating/biasing circuits in the hippocampus, along with the observation of hippocampal phase-coding [Lisman, 2005], Schaffer collateral synaptic dynamics could affect the associative properties of hippocampal computations through transient alterations to the amplitude and phase of the CA1 firing probability. Theoretical and modeling studies indicate that recurrent neural networks may utilize dynamic transients for discrimination and identification [Buckley and Nowotny, 2011; Curto et al, 2012; Rabinovich and Varano, 2011]. Since the Schaffer collateral systematically depolarizes a subset of the CA1 dendrites that receive inputs from the ECIII [Ang et al, 2005], a contextual network selection process could operate transiently by utilizing the interaction between short-term synaptic plastic. ity and the dynamic state of depolarization of the CA1 dendrites [Speed and Dobrunz, 2009; Volman et al, 2009]. Task-dependent transient increases in oscillatory synchronization along with frequency-selective increases in oscillatory coherence are experi- 
mentally supported mechanisms that are consistent with an oscillatory gating hypothesis [Akam and Kullmann, 2010]. Finally, the interaction of synaptic dynamics with the oscillatory modulation of the depolarization potential of excitatory neurons may contribute to phase precession [Thurley et al, 2008], which might affect the magnitude and sign of STDP [Masquelier et al, 2009; Bush et al, 2010], in turn affecting the state of STP [Markram and Tsodyks, 1996; Liebold and Bendels, 2009; Carvalho and Buonomano, 2011].

In the conceptualization of the hippocampal circuit proposed here (Fig. 4.7), the CA1 interprets the dense cortical representation of the spatio-temporal signals from the EC by coordinating predictions from the CA3 and/or CA2 with observations from the EC. The means by which the hippocampus isolates the expected from the observed signals appears to involve the relative timing of the inputs to CA1 from CA3 (and/or CA2) and EC with respect to the theta-band modulation of the local field potential (LFP) [Cutsuridis and Wennekers, 2009; Mizuseki et al, 2009; Jones and McHough, 2011; Hyman et al, 2011]. The wealth of data pointing to a correlation between cognition and timing in the hippocampus [Tort et al, 2009; Uhlhaas and Singer, 2010; Benchenane et al, 2011; Holderith et al, 2011] indicates the sensitivity of the operations involved in interpreting the densely-encoded EC activity. 


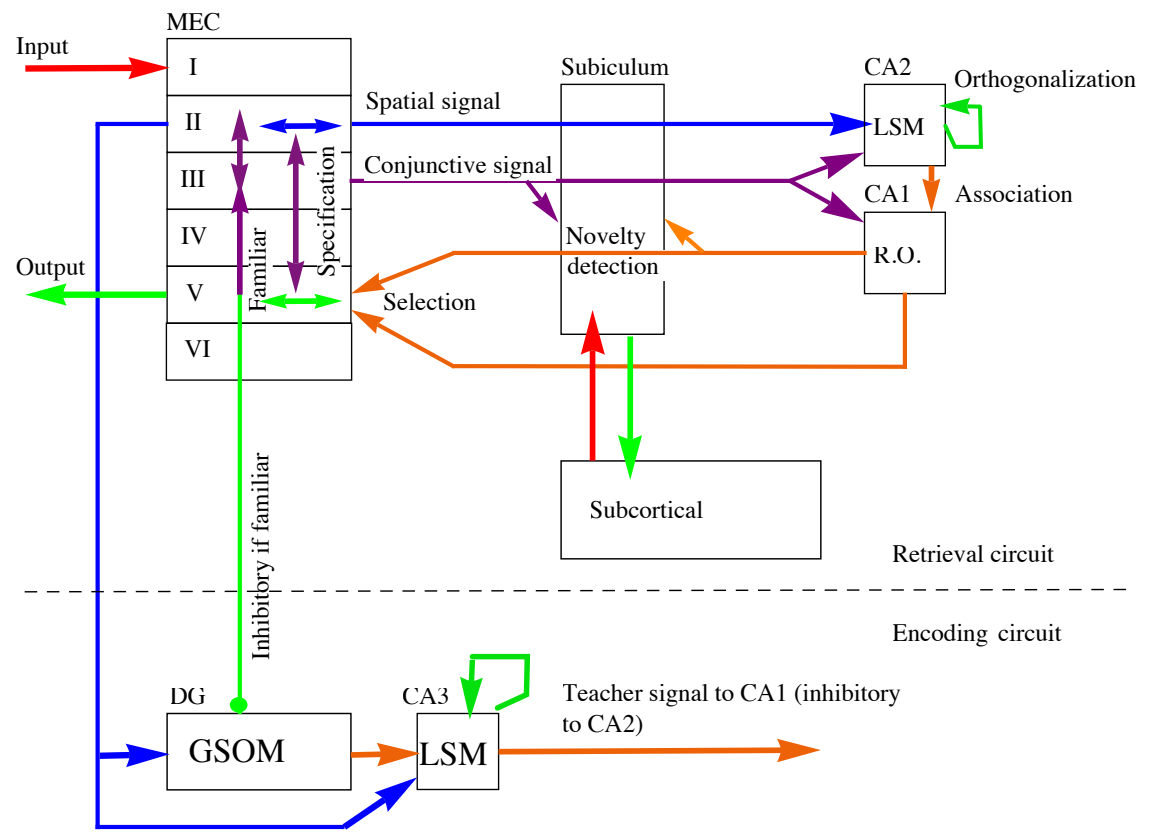

Figure 4.7. Diagram of a proposal for interpreting hippocampal processing. $L S M=$ "Liquid State Machine". R.O. = "Read-Out". GSOM = "Growing Self-Organizing Machine".

To indulge in evolutionary speculation: the dual role of the hippocampus in spatial awareness and the formation of episodic memories [Vann and Albassar, 2011; Stella et al, 2011] might suggest the possibility that contextualized episodic memory organization is an adaptive functionality co-opted from the spatial perception machinery [Whishaw and Wallace, 2003; Behrendt, 2010]. The constraint of topological consistency between the levels of cortical representation that are required for spatial cognition may have provided the selection pressure to drive the formation of the hippocampal-neocortical episodic memory system, while the neocortex diversified and expanded 
[Manns and Eichenbaum, 2006]. Indeed, the neocortex displays great diversity among mammals, while the parahippocampal system remains largely conserved [Eichenbaum and Lipton, 2008]. The speculative proposal that episodic memory organization has its origins in spatial awareness and processing might also gain support from philosophical arguments concerning embodied cognition [Johnson, 2006; Rabinovich and Varano, 2011]. Regardless of whether the function of contextualized episodic memory organization represents a repurposing of the spatial cognition system, the fact that the hippocampus interprets a compressed representation of cortical activity enables operations on the compressed cortical representation (at the level of the EC) to be meaningfully transformed back to the sparse and more permanent representation in the less-connected lobes of the cerebral cortex.

The lack of topological connections over the total space of the neocortex is (probably) a consequence of space limitations resulting in the impossibility of complete neuronal interconnectivity [Klyachko and Stevens, 2003; Raj and Chen, 2011], thus requiring topology-preserving mappings (morphisms) from the neocortex to the entorhinal cortex in order to coherently bind multimodal representations (cognitive percepts) from the sensory and associational cortices at the level of the EC and hippocampus [Dabaghian et al, 2011; Derdikman et al, 2009; Gustafson and Daw, 2011], and the cortex in general [Fiori, 2008]. The projections from neocortex to entorhinal cortex may evolve through a series of compositions,

$$
E=F_{e}\left(F_{p}\left(F_{n}(N)\right)\right),
$$


where $N$ represents the activity at the neocortex, $E$ represents the lower dimensional activity at the entorhinal cortex, and the middle function represents the parahippocampal transformation. At each level multiple output sets converge on the next level. For instance, $F_{n}$ consists of six primary regions, each of which has multiple subregions [Burwell, 2001]. The output from each subregion can be represented by the activity on one set of neurons (the principal neurons), although each subregional set comprises potentially more than one set of neurons if the interneurons are included. Each processing stage could be represented by the complete set of regional output sets. For instance, $F_{n}=\left\{F_{n, 1}, F_{n, 2}, \ldots, F_{n, q}\right\}$, for $q$ regions. The functions describing the output of each regional set of neurons could be written in terms of their component activities:

$$
F_{n, i}=\sum_{j=1}^{m} \boldsymbol{f}_{n, i, j}\left(\mathbf{W}_{\mathrm{post}, n, i, j} \boldsymbol{W}_{\mathrm{pre}, n, i, j}(t)+\boldsymbol{\eta}\right),
$$

where $\mathbf{W}_{\text {post }}$ is the $l \times k$ postsynaptic weight matrix, and $\boldsymbol{W}_{\text {pre }}$ is the $l \times k$ presynaptic weight function, and $k$ is the largest number of synapses on any of the $l$ neurons in the $i^{\text {th }}$ member of the set of $q$ regions. The function $f$ accounts for all electrochemical activity on the neuron, including receptor kinetics, dendritic integration and ion channel currents. The output of $f$ is an $l \times 1$ vector representing the spiking state of the neurons in the $i^{\text {th }}$ set. The array $\boldsymbol{\eta}$ is a guassian process representing the stochasticity of the individual synapses. The sum is over all $m$ sets of input neurons to $F_{n, i}$, including interneurons, modulatory inputs, recurrent inputs, and afferent inputs. $\mathbf{W}_{\text {post }}$ and $\boldsymbol{W}_{\text {pre }}$ are multiplied element-wise. The presynaptic weights are evaluated at time $t$ : 


$$
\boldsymbol{W}_{\text {pre }, n, i, j}(t)=\boldsymbol{g}_{\mathrm{pre}, n, i, j}\left(\boldsymbol{W}_{\mathrm{pre}, n, i, j}\left(t-\boldsymbol{\tau}_{n, i, j}\right), \mathcal{X}_{n, i, j}(t), \boldsymbol{\tau}_{n, i, j}\right),
$$

where $\tau$ are the times since the last spike arrivals at all $l \times k$ synapses on set $i, \mathcal{X}$ is the $l \times$ $k$ array of binary-valued inputs, and $g_{\text {pre }}$ is the function that relates the previous values of presynaptic release probabilities, $\boldsymbol{W}_{\text {pre }, n, i, j}\left(t-\tau_{n, i, j}\right)$, to the current values. The functions $\boldsymbol{f}$ and $\boldsymbol{g}_{\text {pre }}$ have additional parameters, not explicitly listed, some of which may be functions of the states of other variables, but most of which can be considered constant. The values in $\mathbf{W}_{\text {post }}$ are governed by rules that depend on the temporal difference between input and output spikes, and on the state and timing of modulatory inputs.

The reciprocation of anatomical connections through the cortical hierarchy [Lavenex and Amaral, 2000; Agster and Burwell, 2009] allows forward and inverse morphisms between regions, so that the composition of functions in Eqn.(4.1) might also be regarded as a progressive embedding of manifolds, not unlike the manifold learning formulation introduced by [Kramer, 1991]. In this conception, the task of the hippocampus is to learn the topological connections on the lowest dimension submanifold, such that an atlas of charts can be made which preserves the topology of the connections in the total space of the neocortex. The hippocampus also provides a dynamical extension to the operations that can be performed on the low-dimension manifolds [Derdikman and Moser, 2010].

If the CA1 Schaffer collateral (SC) inputs could be linearized, then the stability of the SC synaptic outputs could be determined from the eigenvalues of its presynaptic 
weight matrix [Curto et al, 2012]. The dynamic behavior of the presynaptic weight matrix might be regarded then, as a mechanism to briefly destabilize the CA1 region, which, if already near a bifurcation, could either momentarily separate trajectories [Siri et al, 2008], or push the network into a new attractor state [Cortes et al, 2006] (not to imply that the feedforward CA1 is capable of self-sustaining dynamics, as it lacks recurrence, but that the CA1's response to input patterns from the CA3 could differ based on the state of the SC synapses during the presentation of the inputs). The synaptic depression that follows facilitation in the gamma range could serve to keep the circuit from remaining destabilized, while the frequency dependence of the dynamic weight changes might filter the regimes during which destabilization is operant. Transient destabilization may also fit into the framework of Stable Heteroclinic Orbits (SHO) proposed by Rabinovich and others [Rabinovich et al, 2008; Muezzinoglu et al, 2010]: synaptic dynamics might affect the stability of any given saddle-node in a SHO.

\subsection{Conclusion}

A hypothesis about the function of STP at the Schaffer collateral was developed. Based on the observation that STP provides a narrow band of gain centered around the slow delta rate, the hypothesis states that STP provides a targeted emphasis of the ISIs upon which signals are transmitted to the CA1 from the CA3 during recall. The gain is hypothesized to temporarily destabilize the balance of excitation and inhibition in the CA1, enabling a nonlinear response within the communication band. The hypothesis originally postulated that an STP-enabled response nonlinearity would increase signal 
specificity in CA1. However, given the complexity of the relationship between gamma and theta in the CA1-CA3 regions [Atallah and Scanziani, 2009], it is possible that STP plays a role in the transfer of information between CA subregions, including signalling, triggering, or even accelerating a transition from fast to slow gamma activity [Colgin and Moser, 2010]. Furthermore, since fast and slow gamma are thought to correlate respectively with encoding and retrieval, the coordination of systems that operate on signals carried on these bands may be involved in perceptual processes, as perhaps indicated by interactions between activity on CA1 dendrites in stratum lacunosum-moleculare (distal: from the temporoammonic pathway) and stratum radiatum (more proximal: from the Schaffer collateral) [Takahashi and Magee, 2009; Ang et al, 2005]. Hence, upsetting the coordination of gamma-encoded signals may disturb certain cognitive processes that rely upon the separation of encoding and retrieval for proper functioning. Examples of the types of cognitive pathologies that may involve disruption of gamma encoding include schizophrenia [Uhlhaas and Singer, 2010], autism [Deng et al, 2011] and possibly Alzheimer's [Villette et al, 2010].

The molecular specificity of the DSCI model of STP could enable imbalances of exocytotic proteins to be mapped to synaptic activity. These maps could be used either to predict or to verify experimental results. For example, [Xu et al, 2012] found that syt1-specific knockdown in the hippocampus affected the precision of contextual fear conditioning. This type of result could have far-reaching implications. For instance, since the effect of syt1 knockdown is essentially that of a high-pass filter, this result 
suggests not only the importance of bursting in the hippocampus [Buzsaki, 2012], but also the possibility that syt 2 could operate in the place of syt1. For instance, the failure of syt1-mediated inhibition of dendritic calcium influx can lead to stronger bursting in pyramidal cells [ibid]. In the hippocampus, bursting behavior (defined as three or more spikes within 8ms [Ranck, 1973]) is much more common than in the EC or the mPFC [Fujisawa et al, 2008; Mizuseki et al, 2009]. If the DSCI model is found to reliably predict results like this, then the model may confidently be used as a component of larger models that simulate hippocampal information processing. 


\section{References}

Abbott LF (2008) Theoretical neuroscience rising. Neuron 60:489-495.

Abbott LF, Regehr WG (2004) Synaptic computation. Nature 431:769-803.

Abraham WC, Robins A (2005) Memory retention - the synaptic stability versus plasticity dilemma. Trends Neurosci 28:73-78.

Acsady L, Kali S (2007) Models, structure, function: the transformation of cortical signals in the dentate gyrus. In: Prog Brain Res 163 (Scharfman HE, ed), pp577-599. Elsevier B.V.

Addis DR, Cheng T, Roberts RP, Schacter DL, (2010) Hippocampal contributions to the episodic simulation of specific and general future events. Hippocampus 21:1045-1052.

Agster KL, Burwell RD, (2009) Cortical efferents of the perirhinal, postrhinal, and entorhinal cortices of the rat. Hippocampus 19:1159-1186.

Ahmed OJ, Mehta MR, (2009) The hippocampal rate code: anatomy, physiology and theory. Trends Neurosci 32:329-338.

Aimone JB, Deng W, Gage FH (2011) Resolving new memories: a critical look at the dentate gyrus, adult neurogenesis, and pattern separation. Neuron 70:589-596.

Akam T, Kullmann DM, (2010) Oscillations and filtering networks support flexible routing of information. Neuron 67:308-320.

Akopian G, Walsh JP (2002) Corticostriatal paired-pulse potentiation produced by voltage-dependent activation of NMDA receptors and L-type Ca2+ channels. J Neurophysiol 87:157-165.

Alahakoon D, Halgamuge SK, Srinivasan B (2000) Dynamic self-organizing maps with controlled growth for knowledge discovery. IEEE Trans Neural Netw 11:601-614.

de Almeida L, Idiart M, Lisman JE (2007) Memory retrieval time and memory capacity of the CA3 network: role of gamma frequency oscillations. Learn Mem 14:795-806.

de Almeida LD, Idiart M, Lisman JE (2009) The input-output transformation of the hippocampal granule cells: from grid cells to place fields. J Neurosci 29:7504-7512. 
Amaral D, Lavenex P (2007) Hippocampal neuroanatomy. In: The hippocampus book (Anderson P, Morris R, Amaral D, Bliss T, O'Keefe J, eds), pp37-114. Oxford: Oxford UP.

Amit DJ, Mongillo G (2003) Spike-driven synaptic dynamics generating working memory states. Neural Comput 15:565-596.

Anderson P, Morris R, Amaral D, Bliss T, O'Keefe J (2007) Historical perspective: proposed functions, biological characteristics, and neurobiological models of the hippocampus. In: The hippocampus book (Anderson P, Morris R, Amaral D, Bliss T, O'Keefe J, eds), pp9-36. Oxford: Oxford UP.

Ang CW, Carlson GC, Coulter DA (2005) Hippocampal CA1 circuitry dynamically gates direct cortical inputs preferentially at theta frequencies. J Neurosci 25:9567-9580.

Ang CW, Carlson GC, Coulter DA (2006) Massive and specific dysregulation of direct cortical input to the hippocampus in temporal lobe epilepsy. J Neurosci 26:11850-11856.

Ashby WR (1956) An introduction to cybernetics. London: Chapman \& Hall.

Atallah BV, Scanziani M (2009) Instantaneous modulation of gamma oscillation frequency by balancing excitation with inhibition. Neuron 62:566-577.

Atlas D (2010) Signaling role of the voltage-gated calcium channel as the molecular on/off-switch of secretion. Cell Signal 22:1597-1603.

Atluri PP, Regehr WG (1996) Determinants of the time course of facilitation at the granule cell to Purkinje cell synapse. J Neurosci 16:5661-5671.

Atwood HL, Karunanthi S (2002) Diversification of synaptic strength: presynaptic elements. Nat Rev Neurosci 3:497-516.

Augustine GJ (2001) How does calcium trigger neurotransmitter release? Curr Opin Neurobiol 11:320-326.

Augustine GJ, Santamaria F, Tanaka K (2003) Local calcium signaling in neurons. Neuron 40:331-346.

Averbeck BB, Latham P, Pouget A (2006) Neural correlations, population coding and computation. Nat Rev Neurosci 7:358-366.

Back AD, Chen T (2002) Universal approximation of multiple nonlinear operators by neural networks. Neural Comput 14:2561-2566.

Bahar AS, Shirvalkar PR, Shapiro ML (2011) Memory-guided learning: CA1 and CA3 neuronal ensembles differentially encode the commonalities and differences between situations. J Neurosci 31:12270-12281. 
Baker JL (2003) Is there a support vector machine hiding in the dentate gyrus? Neurocomputing 52:199-207.

Bartesaghi R, Gessi T (2004) Parallel activation of field CA2 and dentate gyrus by synaptically elicited perforant path volleys. Hippocampus 14:948-963.

Bast $\mathrm{T}$ (2011) The hippocampal learning-behavior translation and the functional significance of hippocampal dysfunction in shizophrenia. Curr Opin Neurobiol 21:492-501.

Beed P, Bendels MHK, Wiegand HF, Leibold C, Johenning FW, Schmitz D (2010) Analysis of excitatory microcircuitry in the medial entorhinal cortex reveals cell-typespecific differences. Neuron 68:1059-1066.

Behrendt RP (2010) Contribution of hippocampal region CA3 to consciousness and schizophrenic hallucinations. Neurosci Biobehav Rev 34:1121-1136.

Behrens CJ, van den Boom LP, de Hoz L, Fiedman A, Heinemann U (2005) Induction of sharp wave-ripple complexes in vitro and reorganization of hippocampal networks. Nat Neurosci 8:1560-1567.

Benchenane K, Tiesinga PH, Battaglia FP (2011) Oscillations in the prefrontal cortex: a gateway to memory and attention. Curr Opin Neurobiol 21:475-485.

Benes FM, Kwok EM, Vincent SL, Todtenkopf MS (1998) A reduction of nonpyramidal cells in sector CA2 of schizophrenics and manic depressives. Biol Psychiatry 44:88-97.

Berger TW, Song D, Chan RHM, Marmarelis VZ (2010) The neurobiological basis of cognition: identification by multi-input, multioutput nonlinear dynamical modeling. Proc IEEE 98:356-374.

Bethus I, Tse D, Morris RGM (2010) Dopamine and memory: modulation of the persistence of memory for novel hippocampal NMDA receptor-dependent paired associates. J Neurosci 30:1610-1618.

Berton F, Iborra C, Boudier JA, Seagar MJ, Marqueze B (1997) Developmental regulation of synaptotagmin I, II, III, and IV mRNAs in the rat CNS. J Neurosci 17:1206-1216.

Bhalla A, Tucker WC, Chapman ER (2005) Synaptotagmin isoforms couple distinct ranges of $\mathrm{Ca} 2+, \mathrm{Ba} 2+$, and $\mathrm{Sr} 2+$ concentration to SNARE-mediated membrane fusion. Mol Biol Cell 16:4755-4764.

Billups B, Forsythe ID (2002) Presynaptic mitochondrial calcium sequestration influences transmission at mammalian synapses. J Neurosci 22:5840-5847.

Bischofberger J, Engel D, Frotscher M, Jonas P (2006) Timing and efficacy of transmitter release at mossy fiber synapses in the hippocampal network. Pflugers Arch 
453:361-372.

Bradacs H, Cooper RL, Msghina M, Atwood HL (1997) Differential physiology and morphology of phasic and tonic motor axons in a crayfish limb extensor muscle. J Exp Biol 200:677-691.

Bragin A, Jando G, Nadasdy Z, Hetke J, Wise K, Buzsaki G (1995) Gamma (40-100 $\mathrm{Hz}$ ) oscillation in the hippocampus of the behaving rat. J Neurosci 15:47-60.

Branco T, Staras K (2009) The probability of neurotransmitter release: variability and feedback control at single synapses. Nat Rev Neurosci 10:373-383.

Brown TH, Zador AM (1990) Hippocampus. In: The synaptic organization of the brain, third edition (Shepherd GM, ed), pp346-388. New York: Oxford UP.

Brown TI, Ross RS, Keller JB, Hasselmo ME, Stern CE (2010) Which way was I going? Contextual retrieval supports the disambiguation of well learned overlapping navigational routes. J Neurosci 30:7414-7422.

Brunger AT (2006) Structure and function of SNARE and SNARE-interacting proteins. Quart Rev Biophys 38:1-47.

Brunger AT, Weninger K, Bowen M, Chu S (2009) Single-molecule studies of the neuronal SNARE fusion machinery. Annu Rev Biochem 78:903-928.

Buckley CL, Nowotny T (2011) Transient dynamics between displaced fixed points: an alternate nonlinear dynamical framework for olfaction. Brain Res 1434:62-72.

Buckmaster CA (2004) Entorhinal cortex lesions disrupt the relational organization of memory in monkeys. J Neurosci 24:9811-9825.

Buckner RL (2010) The role of hippocampus in prediction and imagination. Annu Rev Psychol 61:27-48.

Buesing L, Maass W (2010) A spiking neuron as information bottleneck. Neural Comput 22:1961-1992.

Buonomano DV (2000) Decoding temporal information: a model based on short-term synaptic plasticity. J Neurosci 20:1129-1141.

Burgalossi A, Herfst L, von Heimendahl M, Forste H, Haskic K, Schmidt M, Brecht M (2011) Microcircuits of functionally identified neurons in the rat medial entorhinal cortex. Neuron 70:773-786.

Burwell RD (2001) The parahippocampal region: corticocortical connectivity. Ann N Y Acad Sci:25-42.

Bush D, Philippides A, Husbands P, O'Shae M (2010) Dual coding with STDP in a spiking recurrent neural network model of the hippocampus. PLoS Comput Biol 
6:e1000839.

Buzsaki G (2012) How do neurons sense a spike burst? Neuron 73:857-859.

Buzsaki G, Draguhn A (2004) Neural oscillations in cortical networks. Science 304:1926-1929.

Canto CB, Wouterlood FG, Witter MP (2008) What does the anatomical organization of the entorhinal cortex tell us? Neural Plast 2008: article 381243.

Carvalho TP, Buonomano DV (2011) A novel learning rule for long-term plasticity of short-term synaptic plasticity enhances temporal processing. Front Comput Neurosci 5: article 20.

Catterall WA, Few AP (2008) Calcium channel regulation and presynaptic plasticity. Neuron 59:882-901.

Chapman ER (2008) How does synaptotagmin trigger neurotransmitter release? Annu Rev Biochem 77:615-641.

Chawla MK, Guzowski JF, Ramirez-Amaya V, Lipa P, Hoffman KL, Marriott LK, Worley PF, McNaughton BF, Barnes CA (2005) Sparse, environmentally selective expression of Arc RNA in the upper blade of the rodent fascia dentate by brief spatial experience. Hippocampus 15:579-586.

Chevaleyre V, Siegelbaum SA (2010) Strong CA2 pyramidal neuron synapses define a powerful disynaptic cortico-hippocampal loop. Neuron 66:560-572.

Chicka MC, Chapman ER (2009) Concurrent binding of complexin and synaptotagmin to liposome-embedded SNARE complexes. Biochemistry 48:657-659.

Chklovskii DB, Mel BW, Svoboda K (2004) Cortical rewiring and information storage. Nature 431:782-788.

Chrobak JJ, Buzsaki G (1994) Selective activation of deep layer (V-VI) retrohippocampal cortical neurons during hippocampal sharp waves in the behaving rat. J Neurosci 14:6160-6170.

Chrobak JJ, Lorincz A, Buzsaki G (2000) Physiological patterns in the hippocampalentorhinal cortex system. Hippocampus 10:457-465.

Cohen R, Atlas D (2004) R-type voltage-gated Ca2+ channel interacts with synaptic proteins and recruits synaptotagmin to the plasma membrane of Xenopus oocytes. Neuroscience 128:831-841.

Cohen R, Marom M, Atlas D (2007) Depolarization-evoked secretion requires two vicinal transmembrane cysteines of syntaxin 1A. PLoS ONE 2:e1273.

Colgin LL, Moser EI (2010) Gamma oscillations in the hippocampus. Physiology 
25:319-329.

Colgin LL, Moser EI, Moser MB (2008) Understanding memory through hippocampal remapping. Trends Neurosci 31:469-477.

Colgin LL, Leutgeb S, Jezek K, Leutgeb JK, Moser EI, McNaughton BL, Moser MB (2010) Attractor-map versus autoassociation based attractor dynamics in the hippocampal network. J Neurophysiol 104:35-50.

Colombo M, Gross CG (1994) Reponses of inferior temporal cortex and hippocampal neurons during delayed matching to sample in monkeys (Macaca fascicularis). Behav Neurosci 108:443-455.

Cortes JM, Garrido PL, Marro J, Torres JJ (2004) Switching between memories in neural automata with synaptic noise. Neurocomputing 58-60:67-71.

Cortes JM, Torres JJ, Marro J, Garrido PL, Kappen HJ (2006) Effects of fast presynaptic noise in attractor neural networks. Neural Comput 18:614-633.

Cotterill RMJ (2001) Cooperation of the basal ganglia, cerebellum, sensory cerebrum and hippocampus: possible implications for cognition, consciousness, intelligence and creativity. Prog Neurobiol 64:1-33.

Coulter DA, Carlson GC (2007) Functional regulation of the dentate gyrus by GABAmediated inhibition. In: Prog Brain Res 163 (Scharfman HE, ed), pp235-244. Elsevier B.V.

Coulter DA, Yue C, Ang CW, Weissinger F, Goldberg E, Hsu FC, Carlson GC, Takano H (2011) Hippocampal microcircuit dynamics probed using optical imaging approaches. J Physiol 589:1893-1903.

Csicsvari J, Hirase H, Mamiya A, Buzsaki G (2000) Ensemble patterns of hippocampal CA1-CA3 neurons during sharp wave-associated population events. Neuron 28:585-594.

Curto C, Degeratu A, Itzkov V (2012) Flexible memory networks. Bull Math Biol 74:590-614.

Cutsuridis V, Wennekers T (2009) Hippocampus, microcircuits and associative memory. Neural Netw 22:1120-1128.

Dabaghian Y, Cohn AG, Frank L (2011) Topological coding in hippocampus. In: Computational modeling and simulation of intellect: current state and future perspectives (Igelnik B, ed), pp293-320. Hershey PA: Information Science Reference.

Davidson TJ, Kloosterman F, Wilson MA (2009) Hippocampal replay of extended experience. Neuron 63:497-507. 
Davies JN, Zamponi GW (2008) Old proteins, developing roles. Channels 2:130-138.

Davis GW, Murphey RK (1994) Long-term regulation of short-term transmitter release properties: retrograde signaling and synaptic development. Trends Neurosci 17:9-13.

Dayan P, Abbott LF (2001) Theoretical neuroscience: computational and mathematical modeling of neural systems. Cambridge MA: The MIT Press.

Deco G, Rolls ET, Romo R (2010) Synaptic dynamics and decision making. Proc Natl Acad Sci USA 107:7545-7549.

Deng PY, Sojka D, Klyachko VA (2011) Abnormal presynaptic short-term plasticity and information processing in a mouse model of Fragile X Syndrome. J Neurosci 31:10971-10982.

Deng W, Aimone JB, Gage FH (2010) New neurons and new memories: how does adult hippocampal neurogenesis affect learning and memory? Nat Rev Neurosci 11:339-350.

Derdikman D, Moser EI (2010) A manifold of spatial maps in the brain. Trends Cogn Sci 14:561-569.

Derdikman D, Whitlock JR, Tsao A, Fyhn M, Hafting T, Moser, MB, Moser EI (2009) Fragmentation of grid cell maps in a multicompartment environment. Nat Neurosci 12:1325-1332.

Destexhe A, Pare D (1999) Impact of network activity on the integrative properties of neocortical pyramidal neurons in vivo. J Neurophysiol 81:1531-1547.

Destexhe A, Mainen ZF, Sejnowski TJ (1995) Fast kinetic models for simulating AMPA, NMDA, GABA $A$ and GABA $B$ receptors. In: The neurobiology of computation (Bower J, ed), pp9-14. Norwell MA: Kluwer Acedemic Press.

Destexhe A, Mainen ZF, Sejnowski TJ (2001) Kinetic models of synaptic transmission. In: Methods in neuronal modeling: from ions to networks, second edition (Koch C, Segev I, eds), pp1-26. Cambridge MA: The MIT Press.

DeVito LM, Eichenbaum H (2011) Memory for the order of events in specific sequences: contributions of the hippocampus and medial prefrontal cortex. J Neurosci 31:3169-3175.

DeVito LM, Kanter BR, Eichenbaum H (2009a) The hippocampus contributes to memory expression during transitive inference in mice. Hippocampus 20:208-217.

DeVito LM, Konigsberg R, Lykken C, Sauvage M, Young III WS, Eichenbaum H (2009b) Vasopressin 1b receptor knock-out impairs memory for temporal order. J Neurosci 29:2676-2683. 
Dickson CT, Magistretti J, Shalinsky MH, Fransen E, Hasselmo ME, Alonso A (2000) Properties and role of $I \mathrm{~h}$ in the pacing of subthreshold oscillations in entorhinal cortex layer II neurons. J Neurophysiol 83:2562-2579.

Dittman JS, Kreitzer AC, Regehr WG (2000) Interplay between facilitation, depression, and residual calcium at three presynaptic terminals. J Neurosci 20:1374-1385.

Doboli S, Minai AA, Best PJ (2000) Latent attractors: a model for context-dependent place representations in the hippocampus. Neural Comput 12:1009-1043.

Dobrunz LE, Stevens CF (1999) Response of hippocampal synapses to natural stimulation patterns. Neuron 22:157-166.

Dodge FA Jr, Rahamimoff R (1967) Co-operative action a calcium ions in transmitter release at the neuromuscular junction. J Physiol 193:419-432.

Domanska MK, Kiessling V, Stein A, Fasshaur D, Tamm LK (2009) Single vesicle millisecond fusion kinetics reveals number of SNARE complexes optimal for fast SNARE-mediated membrane fusion. J Biol Chem 284:32158-32166.

Duncan K, Ketz N, Inati SJ, Davachi SL (2012) Evidence for area CA1 as a match/mismatch detector: a high-resolution fMRI study of the human hippocampus. Hippocampus 22:389-398.

Durstewitz D (2009) Implications of synaptic biophysics for recurrent network dynamics and active memory. Neural Netw 22:1189-1200.

Egorov AV, Hamam BN, Fransen E, Hasselmo ME, Alonso AA (2002) Graded persistent activity in entorhinal cortex neurons. Nature 420:173-178.

Eichenbaum H (2004) Hippocampus: Cognitive processes and neural representations that underlie declarative memory. Neuron 44:109-120.

Eichenbaum H, Lipton PA (2008) Towards a functional organization of the medial temporal lobe memory system: role of the parahippocampal and medial entorhinal cortical areas. Hippocampus 18:1314-1324.

Eichenbaum H, Dudchencko P, Wood E, Shapiro M, Tanila H (1999) The hippocampus, memory, and place cells: is it spatial memory or memory space? Neuron 23:209-226.

Emes RD, Pocklington AJ, Anderson CNG, Bayes A, Collins MO, Vickers CA, Croning MDR (2008) Evolutionary expansion and anatomical specialization of synapse proteome complexity. Nat Neurosci 11:799-806.

Erchova I, Kreck G, Heinemann U, Herz AVM (2004) Dynamics of rat entorhinal cortex layer II/III cells: characteristics of membrane potential resonance at rest predict oscillation properties near threshold. J Physiol 560:89-110. 
Eschenko O, Ramadan W, Molle M, Born J, Sara SJ (2008) Sustained increase in hippocampal sharp-wave ripple activity during slow-wave sleep after learning. Learn Mem 15:222-228.

Ferguson GD, Wang H, Herschman HR, Storm DR (2004) Altered hippocampal shortterm plasticity and associative memory in synaptotagmin IV (-/-) mice. Hippocampus 14:964-974.

Fiori S (2008) Lie-group-type neural system learning by manifold retractions. Neural Netw 21:1524-1529.

Foldiak P (2002) Sparse coding in the primate cortex. In: The handbook of brain theory and neural networks, second edition (Arbib MA, ed), pp1064-1067. Cambridge MA: The MIT Press.

Fortune ES, Rose GJ (2001) Short-term synaptic plasticity as a temporal filter. Trends Neurosci 24:381-385.

Foster DJ, Wilson MA (2006) Reverse replay of behavioral sequences in hippocampal place cells during the awake state. Nature 440:680-683.

Franzius M, Sprekeler H, Wiskott L (2007) Slowness and sparseness lead to place, head-direction, and spatial-view cells. PLoS Comput Biol 3:1605-1622.

Frerking M, Schulte J, Wiebe SP, Staubli U (2005) Spike timing in CA3 pyramidal cells during behavior: implications for synaptic transmission. J Neurophysiol 94:1528-1540.

Fritzke B (1994) Growing cell structures - a self-organizing network for unsupervised and supervised learning. Neural Netw 7:1441-1460.

Fujisawa S, Amarasingham A, Harrison MT, Buzsaki G (2008) Behavior-dependent short-term assembly dynamics in the medial prefrontal cortex. Nat Neurosci 11:823-833.

Furtak SC, Wei SM, Agster KL, Burwell RD (2007) Functional neuroanatomy of the parahippocampal region in the rat: the perirhinal and postrhinal cortices. Hippocampus 17:709-722.

Fyhn M, Hafting T, Treves A, Moser MB, Moser EI (2007) Hippocampal remapping and grid realignment in entorhinal cortex. Nature 446:190-194.

Gabriel T, Garcia-Perez E, Mahfooz K, Goni J, Martinez-Turrillas R, Perez-Otano I, Lo DC, Wesseling JF (2011) A new kinetic framework for synaptic vesicle trafficking tested in synapsin knock-outs. J Neurosci 31:11563-11577.

Gaffaney JD, Dunning FM, Wang Z, Hui E, Chapman ER (2008) Synaptotagmin C2B domain regulates $\mathrm{Ca} 2+$-triggered fusion in vitro: critical residues revealed by scanning 
alanine mutagenesis. J Biol Chem 283:31763-31775.

Gasparini S, Magee JC (2006) State dependent dendritic computation in hippocampal CA1 pyramidal neurons. J Neurosci 26:288-2100.

Geppert M, Goda Y, Hammer RE, Li C, Rosahl TW, Stevens CF, Sudhof TC (1994) Synaptotagmin I: a major $\mathrm{Ca} 2+$ sensor for transmitter release at a central synapse. Cell 79:717-727.

Gillespie DT (1996) The mathematics of Brownian motion and Johnson noise. Am J Physics 64:225-240.

Goda Y, Sudhof TC (1997) Calcium regulation of neurotransmitter release: reliably unreliable. Curr Opin Cell Biol 9:513-18

Goldman MS, Maldonado P, Abbot LF (2002) Redundancy reduction and sustained firing with stochastic depressing synapses. J Neurosci 22:584-591.

Graupner M (2010) Mechanisms of induction and maintenance of spike-timing dependent plasticity in biophysical synapse models. Front Comput Neurosci 4: article 136.

Green MJ, Uhlhaas PJ, Coltheart M (2005) Context processing and social cognition in schizophrenia. Curr Psychiatr Rev 1:11-22.

Grossberg S (2009) Beta oscillations and hippocampal place cell learning during exploration of novel environments. Hippocampus 19:881-885.

Grover LM, Kim E, Cooke JD (2009) LTP in hippocampal area CA1 is induced by burst stimulation over a broad frequency range centered around delta. Learn Mem 16:69-81.

Gupta AS, van der Meer MAA, Touretsky DS, Redish AD (2010) Hippocampal replay is not a simple function of experience. Neuron 65:695-705.

Gustafson NJ, Daw ND (2011) Grid cells, place cells, and geodesic generalization for spatial reinforcement learning. PLoS Comput Biol 7:e1002235.

Guzowski JF, Knierim JJ, Moser EI (2004) Ensemble dynamics of hippocampal regions CA3 and CA1. Neuron 44:581-584.

Hafting T, Fyhn M, Molden S, Moser MB, Moser EI (2005) Microstructure of a spatial map in the entorhinal cortex. Nature 436:801-806.

Hampson RE, Heyser CJ, Deadwyler SA (1993) Hippocampal cell firing correlates of delayed-match-to-sample performance in the rat. Behav Neurosci 107:715-739.

Hanse E, Gustafson B (2001) Factors explaining heterogeneity in short-term synaptic dynamics of hippocampal glutamatergic synapses in the neonatal rat. J Physiol 537:141-149. 
Harris KD, Hirase H, Leinekugel X, Henze DA, Buzsaki G (2001) Temporal interaction between single spikes and complex spike bursts in hippocampal pyramidal cells. Neuron 32:141-149.

Harris KM, Sultan P (1995) Variation in the number, location and size of synaptic vesicles provides an anatomical basis for the nonuniform probability of release at hippocampal CA1 synapses. Neuropharmacology 34:1387-1395.

Harvey CD, Collman F, Dombeck DA, Tank DW (2009) Intracellular dynamics of hippocampal place cells during virtual navigation. Nature 461:941-946.

Harrison PJ (2004) The hippocampus in schizophrenia: a review of the neuropathological evidence and its pathophysiological implications. Psychopharmacology 174:151-162.

Hashimoto K, Kano M (1998) Presynaptic origin of paired-pulse depression at climbing fibre-Purkinje cell synapses in the rat cerebellum. J Physiol 506:391-405.

Hasselmo ME (2005) The role of hippocampal regions CA3 and CA1 in matching entorhinal input with retrieval and associations between objects and context: theoretical comments on Lee et al. (2005). Behav Neurosci 119:342-345.

Hasselmo ME, Eichenbaum H (2005) Hippocampal mechanisms for the context-dependent retrieval of episodes. Neural Netw 18:1172-1190.

Hasselmo ME, McClelland JL (1999) Neural models of memory. Curr Opin Neurobiol 9:184-188.

Hasselmo ME, Bodelon C, Wyble B (2002) A proposed function for hippocampal theta rhythm: seperate phases of encoding and retrieval of prior learning. Neural Comput 14:793-817.

Hasselmo ME, Schnell E, Barkai E (1995) Dynamics of learning and recall at excitatory recurrent synapses and cholinergic modulation in rat hippocampal region CA3. J Neurosci 15:5249-5262.

Hasselmo ME, Giocomo LM, Brandon MP, Yoshida M (2010) Cellular dynamical mechanisms for encoding the time and place of events along spatiotemporal trajectories in episodic memory. Behav Brain Res 215:261-274.

Haykin S (1999) Neural networks: a comprehensive foundation. Upper Saddle River NJ: Prentice Hall.

Helmchen F, Borst JGG, Sakmann B (1997) Calcium dynamics associated with a single action potential in a CNS presynaptic terminal. J Biophysics 72:1458-1471.

Hill AJ (1978) First occurrence of hippocampal spatial firing in a new environment. Exp Neurol 62:282-297. 
Hirano T, Best P, Olds J (1970) Units during habituation, discrimination learning, and extinction. Electroencephalogr Clin Neurophysiol 28:127-135.

Hirase H, Recce M (2011) What autoassociative network models may tell us about the neurobiology of memory. Network: Comput Neural Syst 22:231-250.

Hirsch R (1974) The hippocampus and contextual retrieval of information from memory: a theory. Behav Biol 12:421-444.

Hjelmstad GO, Nicoll RA, Malenka RC (1997) Synaptic refractory period provides a measure of probability of release in the hippocampus. Neuron 19:1309-1318.

Hjelmstad GO, Isaac JTR, Nicoll RA, Malenka RC (1999) Lack of AMPA receptor desensitization during basal synaptic transmission in the hippocampal slice. J Neurophysiol 81:3096-3099.

Holderith N, Nemeth B, Papp OI, Veres JM, Nagy GA, Hajos N (2011) Cannabinoids attenuate hippocampal gamma oscillations by suppressing excitatory synaptic input onto CA3 pyramidal neurons and fast spiking basket cells. J Physiol 589:4921-4934.

Hopfield JJ (1982) Neural networks and physical systems with emergent collective computational abilities. Proc Natl Acad Sci USA 79:2554-2558.

Hopfield JJ (1999) Brain, neural networks, and computation. Rev Mod Phys 71:S431S437.

Hosoi N, Sakaba T, Neher E (2007) Quantitative analysis of calcium-dependent vesicle recruitment and its functional role at the calyx of Held synapse. J Neurosci 27:14286-14298.

Howard LR, Kumaran D, Olafsdottir HF, Spiers HJ (2011) Double dissociation between hippocampal and parahippocampal responses to object-background context and scene novelty. J Neurosci 31:5253-5261.

Hua Y, Scheller RH (2001) Three SNARE complexes cooperate to mediate membrane fusion. Proc Natl Acad Sci USA 98:8065-8070.

Hui E, Bai J, Wang P, Sugimori M, Llinas RR, Chapman ER (2005) Three distinct kinetic groupings of the synaptotagmin family: candidate sensors for rapid and delayed exocytosis. Proc Natl Acad Sci USA 102:5210-5214.

Hyman JM, Hasselmo ME, Seamans JK (2011) What is the functional relevance of prefrontal cortex entrainment to hippocampal theta rhythms? Front Neurosci 5: article 24.

Iijima T, Witter MP, Ichikawa M, Tominanga T, Kajiwara R, Matsumoto G (1996) Entorhinal-hippocampal interactions revealed by real-time imaging. Science 272:1176-1179. 
Ito HT, Schuman EM (2012) Functional division of hippocampal area CA1 via modulatory gating of entorhinal cortical inputs. Hippocampus 22:372-387.

Izhekevich E (2006) Dynamical systems in neuroscience: the geometry of excitability and bursting. Cambridge MA: The MIT Press.

Jackson J, Goutagny R, Williams S (2011) Fast and slow gamma rhythms are intrinsically and independently generated in the subiculum. J Neurosci 31:12104-12117.

Jahn R, Lang T, Sudhof TC (2003) Membrane fusion. Cell 112:519-533.

Jarvis SE, Zamponi GW (2005) Masters or slaves? Vesicle release machinery and the regulation of presynaptic calcium channels. Cell Calcium 37:483-488.

Jeffery KJ (2011) Place cells, grid cells, attractors, and remapping. Neural Plasticity 2011: article 182602 .

Jerman T, Kesner RP, Hunsaker MR (2006) Disconnection analysis of CA3 and DG in mediating encoding but not retrieval in a spatial maze learning task. Learn Mem 13:458-464.

Jezek K, Henrikson EJ, Treves A, Moser EI, Moser MB (2011) Theta-paced flickering between place-cell maps in the hippocampus. Nature 478:246-249.

Johnson M (2006) Mind incarnate: from Dewey to Damasio. Daedelus 135:46-54.

Jones MW, McHugh TJ (2011) Updating hippocampal representations: CA2 joins the circuit. Trends Neurosci 34:526-535.

Kaiser M, Varier S (2011) Evolution and development of brain network: from Caenorhabditis elegans to Homo sapiens. Network: Comput Neural Syst 22:143-147.

Kandaswamy U, Deng PY, Stevens CF, Klyachko VA (2010) The role of presynaptic dynamics in processing of natural spike trains in hippocampal synapses. $\mathrm{J}$ Neurosci 30:15904-15914.

Kandel ER (2006) In search of memory: the emergence of a new science of mind. New York: WW Norton \& Company.

Kandel ER, Schwartz JH, Jessell TM (1991) Principles of neural science, 3rd ed. Appleton and Lange.

Kanerva P (1993) Sparse distributed memory and related models. In: Associative neural memories: theory and implementation (Hassoun MH, ed.), pp50-76. New York: Oxford UP.

Karlsson MP, Frank LM (2008) Network dynamics underlying the formation of sparse, informative representations in the hippocampus. J Neurosci 28:14271-14281.

Kesner RP (2007) A behavioral analysis of dentate gyrus function. In: Prog Brain Res 
163 (Scharfman HE, ed), pp567-576. Elsevier B.V.

Kesner RP, Hunsaker MR, Warthen MW (2008) The CA3 subregion of the hippocampus is critical for episodic memory processing by means of relational encoding in rats. Behav Neurosci 122:1217-1225.

van Kesteren MTR, Ruiter DJ, Fernandez G, Hensen RN (2012) How schema and novelty augment memory formation. Trends Neurosci 35:21-219.

Kim DK, Catterall WA (1997) Ca2+-dependent and -independent interactions of the isoforms of the $\alpha 1 \mathrm{~A}$ subunit of brain $\mathrm{Ca} 2+$ channels with presynaptic SNARE proteins. Proc Natl Acad Sci USA 94:14782-14786.

Kim JJ, Fanselow MS (1992) Modality-specific retrograde amnesia of fear. Science 256:675-677.

Kimble DP (1968) Hippocampus and internal inhibition. Psychol Bull 70:285-295.

King MJR, Atwood HL, Govind CK (1996) Structural features of crayfish phasic and tonic neuromuscular terminals. J Comp Neurol 372:618-626.

Klausberger T, Somogyi P (2008) Neuronal diversity and temporal dynamics: the unity of hippocampal circuit operations. Science 321:53-57.

Klyachko VA, Stevens CF (2003) Connectivity optimization and the positioning of cortical areas. Proc Natl Acad Sci USA 100:7937-7941.

Klyachko VA, Stevens CF (2006) Excitatory and feedforward inhibitory hippocampal synapses work synergistically as an adaptive filter of natural spike trains. PLoS Biol 4:1187-1200.

Knierim JJ, Lee I, Hargreaves EL (2006) Hippocampal place cells: parallel input streams, subregional processing, and implications for episodic memory. Hippocampus 16:755-764.

Koch C (1999) Biophysics of computation: information processing in single neurons. New York: Oxford UP.

Kochubey O, Schneggenburger R (2011) Synaptotagmin increases the dynamic range of synapses by driving $\mathrm{Ca} 2+-$ evoked release and by clamping a near-linear remaining $\mathrm{Ca} 2+$ sensor. Neuron 69:736-748.

Kochubey O, Lou X, Schneggenburger R (2011) Regulation of transmitter release by $\mathrm{Ca} 2+$ and synaptotagmin: insights from a large CNS synapse. Trends Neurosci 34:237-246.

Koester HJ, Sakmann B (2000) Calcium dynamics associated with action potentials in single nerve terminals of pyramidal cells in layer $2 / 3$ of the young rat neocortex. J Physiol 529:625-646. 
Kohonen T (1993) Physiological interpretation of the self-organizing map algorithm. Neural Netw 6:895-905.

Komorowski RW, Manns JR, Eichenbaum H (2009) Robust conjunctive item-place coding by hippocampal neurons parallels learning what happens where. J Neurosci 29:9918-9929.

Kramer MA (1991) Nonlinear principal component analysis using autoassociative neural networks. J Am Inst Chem Eng 37:233-243.

Kumaran D, Maguire EA (2007) Which computational mechanisms operate in the hippocampus during novelty detection? Hippocampus 17:735-748.

Kunec S, Hasselmo ME, Kopell N (2005) Encoding and retrieval in the CA3 region of hippocampus: a model of theta phase separation. J Neurophysiol 94:70-82.

Kviem O, Setekleiv J, Kaada BR (1964) Differential effects of hippocampal lesions on maze and passive avoidance learning in rats. Exp Neurol 9:59-72.

Lapedes A, Farber R (1986) A self-optimizing, nonsymmetrical neural net for content addressable memory and pattern recognition. Physica D 22:247-259.

Lavenex P, Amaral DG (2000) Hippocampal-neocortical interaction: a hierarchy of associativity. Hippocampus 10:420-430.

Lee CCJ, Anton M, Poon CS, McRae GJ (2009) A kinetic model unifying presynaptic short-term facilitation and depression. J Comput Neurosci 26:459-473.

Lee I, Kesner RP (2004) Encoding versus retrieval of spatial memory: double dissociation between the dentate gyrus and the perforant path inputs into CA3 in the dorsal hippocampus. Hippocampus 14:66-76.

Lee I, Solivan F (2010) Dentate gyrus is necessary for disambiguating similar objectplace representations. Learn Mem 17:252-258.

Lee I, Hunsaker MR, Kesner RP (2005) The role of hippocampal subregions in detecting spatial novelty. Behav Neurosci 119:145-153.

Lee JS, Ho WK, Lee SH (2012) Actin-dependent rapid recruitment of reluctant synaptic vesicles into a fast-releasing vesicle pool. Proc Natl Acad Sci USA:E765-E774.

Legenstein R, Maass W (2007) Edge of chaos and prediction of computational performance for neural circuit models. Neural Netw 20:323-324.

Lendaris GG (2009) Adaptive dynamic programming approach to experience-based systems identification and control. Neural Netw 22:822-832.

Leutgeb S, Leutgeb JK (2007) Pattern separation, pattern completion, and new neuronal codes within a continuous CA3 map. Learn Mem 14:745-757. 
Leutgeb S, Leutgeb JK, Treves A, Moser MB, Moser EI (2004) Distinct ensemble codes in hippocampal areas CA3 and CA1. Science 305:1295-1298.

Leutgeb S, Leutgeb JK, Moser MB, Moser EI (2005) Place cells, spatial maps and the population code for memory. Curr Opin Neurobiol 15:738-746.

Lever C, Burton S, Jeewajee A, Wills TJ, Cacucci F, Burgess N, O'Keefe J (2010) Environmental novelty elicits a later theta phase of firing in CA1 but not subiculum. Hippocampus 20:229-234.

Levy WB, Wu X, Baxter RA (1995) Unification of hippocampal function via computational/encoding considerations. Int J Neural Syst 6:71-80.

Li F, Pincet F, Perez E, Eng WS, Melia TJ, Rothman JE, Tareste D (2007) Energetics and dynamics of SNAREpin folding across lipid bilayers. Nat Struct Mol Biol 14:890-896.

Li S, Cullen WK, Anwyl R, Rowan MJ (2003) Dopamine-dependent facilitation of LTP induction in hippocampal CA1 by exposure to spatial novelty. Nat Neurosci 6:526-532.

Liaw JS, Berger TW (1999) Dynamic synapse: harnessing the computing power of synaptic dynamics. Neurocomputing 26-27:199-206.

Liaw JS, Xie X, Ghaffari T, Baudry M, Chauvet GA, Berger TW (2000) Role of synaptic geometry in the dynamics and efficacy of synaptic transmission. In: Advances in synaptic plasticity (Baudry M, Davis JL, Thompson RF, eds), pp103-142. Cambridge MA: MIT Press.

Liebold C, Bendels MHK (2009) Learning to discriminate through long-term changes of dynamical synaptic transmission. Neural Comput 21:3408-3428.

Lisman J (1999) Relating hippocampal circuitry to function: recall of memory sequences by reciprocal dentate-CA3 interaction. Neuron 22:233-242.

Lisman J (2005) The theta/gamma discrete phase code occurring during the hippocampal phase precession may be a more general brain coding scheme. Hippocampus 15:913-922.

Lisman J (2009) The pre/post LTP debate. Neuron 63:281-284.

Lisman JE, Otmakova NA (2001) Storage, recall, and novelty detection of sequences by the hippocampus: elaborating on the SOCRATIC model to account for normal and abberant effects of dopamine. Hippocampus 11:551-568.

Lisman J, Redish AD (2009) Prediction, sequences, and the hippocampus. Philos Trans R Soc Lond B Biol Sci 364:1193-1201. 
Lisman JE, Talamini LM, Raffone A (2005) Recall of memory sequences by interaction of the dentate and CA3: a revised model of the phase precession. Neural Netw 18:1191-1201.

Lisman J, Grace AA, Duzel E (2011) A neoHebbian framework for episodic memory; role of dopamine-dependent lat LTP. Trends Neurosci 34:536-547.

Llinas R (1999) The squid giant synapse: a model for chemical transmission. Oxford: Oxford UP.

Ljung L (1987) System identification: theory for the user. Englewood Cliffs NJ: Prentice-Hall.

Lorincz A, Buzsaki G (2001) Two-phase computational model training long-term memories in the entorhinal-hippocampal region. Ann N Y Acad Sci 911:83-111.

Lorincz A, Szirtes G (2009) Here and now: how time segments may become events in the hippocampus. Neural Netw 22:738-747.

Maass W (2010) Liquid state machines: motivation, theory, and applications. In: Computability in context: computation and logic in the real world (Cooper B, Sorbi A, eds), pp275-296. London: Imperial College Press.

Maass W, Markram H (2002) Synapses as dynamic memory buffers. Neural Netw 15:155-161.

Maass W, Sontag ED (2000) Neural systems as nonlinear filters. Neural Comput 12:1743.

Macaluso E, Driver J (2005) Multisensory spatial interactions: a window into functional integration in the human brain. Trends Neurosci 28:265-271.

Magee JC (2000) Dendritic integration of excitatory synaptic input. Nat Rev Neurosci 1:181-190.

Manns JR, Eichenbaum H (2006) Evolution of declarative memory. Hippocampus 16:795-808.

Markram H, Tsodyks M (1996) Redistribution of synaptic efficacy between neocortical pyramidal neurons. Nature 382:807-810.

Markram H, Lubke J, Frotscher M, Sakmann B (1997) Regulation of synaptic efficacy by coincidence of postsynaptic APs and EPSPs. Science 275:213-15.

Markus H (1977) Self-schemata and processing information about the self. J Pers Soc Psychol 35:63-78.

Marr D (1971) Simple memory: a theory for archicortex. Philos Trans R Soc Lond B Biol Sci 262:23-81. 
Marro J, Garrido PL, Torres, JJ (1998) Effect of correlated fluctuations of synapses in the performance of neural networks. Phys Rev Lett 81:2827-2830.

Marro J, Torres JJ, Cortes JM (2007) Chaotic hopping between attractors in neural networks. Neural Netw 20:230-235.

Martin JH (2012) The limbic system and cerebral circuits for reward, emotions, and memory. In: Neuroanatomy: text and atlas, fourth edition, pp 385-414. McGraw-Hill.

Masquelier T, Hugues E, Deco G, Thorpe SJ (2009) Oscillations, phase-of-firing coding, and spike timing-dependent plasticity: an efficient learning scheme. J Neurosci 29:13484-13493.

Maximov A, Sudhof TC (2005) Autonomous function of synaptotagmin 1 in triggering synchronous release independent of asynchronous release. Neuron 48:547-554.

Maximov A, Lao Y, Li H, Chen X, Rizo J, Sorenson JB, Sudhof TC (2008) Genetic analysis of synaptotagmin-7 function in synaptic vesicle exocytosis. Proc Natl Acad Sci USA 105:3986-3991.

Maximov A, Tang J, Yang X, Pang ZP, Sudhof TC (2009) Complexin controls the force transfer from SNARE complexes to membranes in fusion. Science 323:516-521.

Mayor J, Gerstner W (2004) Transient information flow in a network of excitatory and inhibitory neurons: role of noise and signal autocorrelation. J Physiol Paris 98:417-428.

McClelland JL, Goddard NH (1996) Considerations arising from a complementary learning systems perspective on hippocampus and neocortex. Hippocampus 6:654-665.

McGaughy J, Koene RA, Eichenbaum H, Hasselmo ME (2005) Cholinergic deafferentiation of the entorhinal cortex in rats impairs encoding of novel but not familiar stimuli in a delayed nonmatch-to-sample task. J Neurosci 25:10273-10281.

McHugh TJ, Tonegawa S (2009) CA3 NMDA receptors are required for the rapid formation of a salient contextual association. Hippocampus 19:1153-1158.

McNaughton N (2006) The role of the subiculum within the behavioural inhibition system. Behav Brain Res 174:232-250.

Meinrenken CJ, Borst JGG, Sakmann B (2003) Local routes revisited: the space and time dependence of the $\mathrm{Ca} 2+$ signal for phasic transmitter release at the rat calyx of Held. J Physiol 547:665-689.

Mejias JF, Torres JJ (2007) Improvement of spike coincidence detection with facilitating synapses. Neurocomputing 70:2026-2029.

Meyer K (2012) Another remembered present. Science 335:415-416.

Millar AG, Bradacs H, Charlton MP, Atwood HL (2002) Inverse relationship between 
release probability and readily releasable vesicles in depressing and facilitation synapses. J Neurosci 22:9661-9667.

Miller G (2009) On the origin of the nervous system. Science 325:24-26.

Mizumori SJY (2006) Hippocampal place fields: a neural code for episodic memory? Hippocampus 16:685-690.

Mizuseki K, Sirota A, Pastalkova E, Buzsaki G (2009) Theta oscillations provide temporal windows for local circuit computation in the entorhinal-hippocampal loop. Neuron 64:267-280.

Mohrmann R, de Wit H, Verhage M, Neher E, Sorensen JB (2010) Fast vesicle fusion in living cells requires at least three SNARE complexes. Science 330:502-505.

Mole VLD, Araujo AFR (2010) Growing self-organizing surface map: learning a surface topology from a point cloud. Neural Comput 22:689-729.

Montgomery SM, Buzsaki G (2007) Gamma oscillations dynamically couple hippocampal CA 3 and CA1 regions during memory task performance. Proc Natl Acad Sci USA 104:14495-14500.

Morris R (2007) Theories of hippocampal function. In: The hippocampus book (Anderson P, Morris R, Amaral D, Bliss T, O'Keefe J, eds), pp581-714. Oxford: Oxford UP.

Moser EI, Kropff E, Moser MB (2008) Place cells, grid cells, and the brain's spatial representation system. Annu Rev Neurosci 31:69-89.

Msghina M, Govind CK, Atwood HL (1997) Synaptic structure and transmitter release in crustacean phasic and tonic motor neurons. J Neurosci 18:1374-1382.

Muezzinoglu MK, Tristan I, Huerta R, Afraimovich VS, Rabinovich MI (2010) Transients versus attractors in complex networks. Int J Bifur Chaos 20:1653-1675.

Muller M, Felmy F, Schwaller B, Schneggenburger R (2007) Parvalbumin is a mobile presynaptic $\mathrm{Ca} 2+$ buffer in the calyx of Held that accelerates the decay of $\mathrm{Ca} 2+$ and short-term facilitation. J Neurosci 27:2261-2271.

Myers CE, Scharfman HE (2010) Pattern separation in the dentate gyrus: a role for the CA3 backprojection. Hippocampus 21:1190-1215.

Nadel L, Hardt O (2010) Update on memory systems and processes. Neuropsychopharmacology 36:251-273.

Nadel L, Willner J, Kurz EM (1985) Cognitive maps and environmental context. In: Context and learning (Balsam P, Tomie A, eds), pp385-406. Hillsdale NJ: Lawrence Erlbaum Associates. 
Nadkarni S, Bartol TM, Sejnowski TJ, Levine H (2010) Modeling vesicular release at hippocampal synapses. PLoS Comput Biol 6:e1000983.

Nakashiba T, Young JZ, McHugh TJ, Buhl DL, Tonegawa S (2008) Transgenic inhibition of synaptic transmission reveals role of CA3 output in learning. Science 319:1260-1264.

Nakazawa K, Quirk MC, Chitwood RA, Watanabe M, Yekel MF, Sun LD, Kato A, Carr CA, Johnston D, Wilson MA, Tonegawa S (2002) Requirement for hippocampal CA3 NMDA receptors in associative memory recall. Science 297:211-218.

Naraghi M, Neher E (1997) Linearized buffered Ca2+ diffusion in microdomains and its implications for calculation of $[\mathrm{Ca} 2+]$ at the mouth of a calcium channel. J Neurosci 17:6961-6973.

Natschlager T, Maass W, Zador A (2001) Efficient temporal processing with biologically realistic dynamic synapses. Network: Comput Neural Syst 12:75-87.

Neher E, Augustine G (1992) Calcium gradients and buffers in bovine chromaffin cells. J Physiol 450:273-301.

Neher E, Sakaba T (2008) Multiple roles of calcium ions in the regulation of neurotrans. mitter release. Neuron 59:861-872.

Nelson SB, Turrigiano GG (2008) Strength through diversity. Neuron 60:477-482.

Nguyen PV, Kandel ER (1997) Brief theta-burst stimulation induces a transcriptiondependent late phase of LTP requiring cAMP in area CA1 of the mouse hippocampus. Learn Mem 4:230-243.

Niebur E (2007) Generation of synthetic spike trains with defined pairwise correlations. Neural Comput 19:1720-1738.

O'Keefe J (2007) Hippocampal neurophysiology in the behaving animal. In: The hippocampus book (Anderson P, Morris R, Amaral D, Bliss T, O'Keefe J, eds), pp475-548. Oxford: Oxford UP.

O'Keefe J, Dostrovsky J (1971) The hippocampus as a spatial map: preliminary evidence from unit activity in the freely moving rat. Brain Res 34:171-175.

O'Keefe J, Nadel L (1978) The hippocampus as a cognitive map. Oxford: Oxford UP.

O'Mara S (2006) Controlling hippocampal output: the central role of subiculum in hippocampal information processing. Behav Brain Res 174:304-312.

O'Neill J, Pleydell-Bouverie B, Dupret D, Csicsvari J (2010) Play it again: reactivation of waking experience and memory. Trends Neurosci 33:220-229.

Ohliger-Frerking P, Wiebe SP, Staubli U, Frerking M (2003) GABAB receptor-medi- 
ated presynaptic inhibition has history-dependent effects on synaptic transmission during physiologically relevant spike trains. J Neurosci 23:4809-4814.

Onodera H, Sato G, Kogure K (1986) Lesions to Schaffer collaterals prevent ischemic death of CA1 pyramidal cells. Neurosci Lett 68:169-174.

Otsu Y, Shahrezaei V, Li B, Raymond LA, Delaney KR, Murphy TH (2004) Competition between phasic and asynchronous release for recovered synaptic vesicles at developing hippocampal autaptic synapses. J Neurosci 24:420-433.

Pan P, Zucker RA (2009) A general model of synaptic transmission and short-term plasticity. Neuron 62:539-554.

Pantic L, Torres JJ, Kappen HJ, Gielen S (2002) Association memory with dynamic synapses. Neural Comput 12:2903-2923.

Pawlak V, Wickens JR, Kirkwood A, Kerr JND (2010) Timing is not everything: neuromodulation opens the STDP gate. Front Syn Neurosci 2: article 146.

Pfister JP, Dayan P, Mate L (2010) Synapses with short-term plasticity are optimal estimators of presynaptic membrane potentials. Nat Neurosci 13:1271-1275.

Phelps EA (2004) Human emotion and memory: interactions of the amygdala and hippocampal complex. Curr Opin Neurobiol 14:198-202.

Phillips WA, Singer W (1997) In search of common foundations for cortical computation. Behav Brain Sci 20:657-722.

Piaget (1972) The principals of genetic epistemology. New York: Basic Books, Inc.

Pinsky PF, Rinzel J (1994) Intrinsic and network rhythmogenesis in a reduced Traub model for CA3 neurons. J Comput Neurosci 1:39-60.

Poirier GL, Amin E, Aggleton JP (2008) Qualitatively different hippocampal subfield engagement emerges with mastery of a spatial memory task by rats. J Neurosci 28:1034-1045.

Popov SV, Poo M (1993) Synaptotagmin: a calcium-sensitive inhibitor of exocytosis? Cell 73:1247-1249.

Prokhorov DV, Feldkamp LA, Tyukin IY (2002) Adaptive behavior with fixed weights in RNN: an overview. IJCNN'02 3:2018-2022.

Quilichini P, Sirota A, Buzsa'ki G (2010) Intrinsic circuit organization and thetagamma oscillation dynamics in the entorhinal cortex of the rat. J Neurosci 30:11128-11142.

Rabinovich M, Varona P (2011) Robust transient dynamics and brain functions. Front Comput Neurosci 5: article 24. 
Rabinovich M, Huerta R, Laurent G (2008) Transient dynamics for neural processing. Science 321:48-50.

Raj A, Chen Y (2011) The wiring economy principle: connectivity determines anatomy in the human brain. PLoS ONE 6:e14832.

Ranck JB Jr (1973) Studies on single neurons in dorsal hippocampus formation and septum in unrestrained rats: part I. Behavioral correlates and firing repertoires. Exp Neurol 41:462-531.

Ramamoorthi K, Fropf R, Belfort GM, Fitzmaurice HL, McKinney RM, Neve RL, Otto T, Lin Y (2011) Npas4 regulated a transcriptional program in CA3 required for contextual memory formation. Science 334:1669-1675.

Reber TP, Leuchinger R, Boesiger P, Henke K (2012) Unconscious relational inference recruits the hippocampus. J Neurosci 32:6138-6148.

Regehr WG, Carey MR, Best AR (2009) Activity-dependent regulation of synapses by retrograde messengers. Neuron 63:154-170.

Rieke F, Warland D, van Steveninck RR, Bialek W (1997) Spikes: exploring the neural code. Cambridge MA: The MIT Press.

Rizo J, Rosenmund C (2008) Synaptic vesicle fusion. Nat Struct Mol Biol 15:665-674.

Roberts PD, Leen TK (2010) Anti-Hebbian spike-timing-dependent plasticity and adaptive sensory processing. Front Comput Neurosci 4: article 146.

Roberts WM (1994) Localization of calcium signals by a mobile calcium buffer in frog saccular hair cells. J Neurosci 14:3246-3262.

Rogers JL, Kesner RP (2003) Cholinergic modulation of the hippocampus during encoding and retrieval. Neurobiol Learn Mem 80:332-342.

Rogers JL, Kesner RP (2004) Cholinergic modulation of the hippocampus during encoding and retrieval of tone/shock-induced fear conditioning. Learn Mem 11:102-107.

Rolls ET (1989) Functions of neural networks in the hippocampus and neocortex. In: Memory in neural models of plasticity (Byrne JH, Berry WO, eds), pp240-265. San Diego: Academic Press.

Rolls ET (1996) A theory of hippocampal function in memory. Hippocampus 6:601-620.

Rolls ET (2007) An attractor network in the hippocampus: theory and neurophysiology. Learn Mem 14:714-731.

Rolls ET (2010) A computational theory of episodic memory formation in the hip- 
pocampus. Behav Brain Res 215:180-196.

Rolls ET, Deco G (2010) The noisy brain: stochastic dynamics as a principle of brain function. Oxford: Oxford UP.

Rolls ET, Kesner RP (2006) A computational theory of hippocampal function, and empirical tests of the theory. Prog Neurobiol 79:1-48.

Rolls ET, Treves A (1998) Neural networks and brain function. Oxford: Oxford UP.

Rolls ET, Stringer SM, Elliot T (2006) Entorhinal cortex grid cells can map to hippocampal place cells by competitive learning. Network: Comput Neural Syst 17:447-465.

van Rossum MCW (2001) A novel spike distance. Neural Comput 13:751-763.

Rotman Z, Deng PY, Klyachko VA (2011) Short-term plasticity optimizes synaptic information transmission. J Neurosci 31:14800-14809.

Roweis ST, Saul LK (2000) Nonlinear dimensionality reduction by locally linear embedding. Science 290:2323-2326.

Rudy JW, O'Reilly RC (1999) Contextual fear conditioning, conjunctive representations, pattern completion, and the hippocampus. Behav Neurosci 113:867-880.

Rudy JW, Barrientos RM, O'Reilly RC (2002) Hippocampal formation supports conditioning to memory of a context. Behav Neurosci 116:530-538.

Rusakov DA (2006) Ca2+-dependent mechanisms of presynaptic control at central synapses. The Neuroscientist 12:317-326.

Sakaba T (2006) Roles of the fast-releasing and the slowly releasing vesicles in synaptic transmission at the calyx of Held. J Neurosci 26:5863-5871.

Sakaba T, Neher E (2001) Calmodulin mediates rapid recruitment of fast-releasing synaptic vesicles at a calyx-type synapse. Neuron 32:1119-1131.

Sakarya O, Armstrong KA, Adamska M, Wang IF, Tidor B, Degnan BM, Oakley TH, Kosik KS (2007) A post-synaptic scaffold at the origin of the animal kingdom. PLoS ONE 6:e506.

Samsonovich A, McNaughton B (1997) Path integration and cognitive mapping in a continuous attractor neural network model. J Neurosci 17:5900-5920.

Samuel W, Masliah E, Brush DE, Garcia-Munoz M, Patino P, Young SJ, Groves PM (1997) Lesions in the dentate hilum and CA2/CA3 regions of the rat hippocampus produce cognitive deficits that correlate with site-specific glial activation. Neurobiol Learn Mem 68:103-116.

Santiago RA (2004) Context discerning multifunction networks: reformulating fixed 
weight neural networks. IJCNN'04:\#1743.

Saraswati S, Adolfsen B, Littleton JT (2007) Characterization of the role of the synaptotagmin family as calcium sensors in facilitation and asynchronous release. Proc Natl Acad Sci USA 104:14122-14127.

Sargolini F, Fyhn M, Hafting T, McNaughton BL, Witter MP, Moser MB, Moser EI (2006) Conjunctive representation of position, direction, and velocity in entorhinal cortex. Science 312:758-762.

Sasaki T, Matsuki N, Ikegaya Y (2007) Metastability of active CA3 networks. J Neurosci 27:517-528.

Scharfman HE (2007) The CA3 "backprojection" to the dentate gyrus. Prog Brain Res 163 (Scharfman HE, ed), pp627-637. Elsevier B.V.

Schonn JS, Maximov A, Lao Y, Sudhof TC, Sorensen JB (2008) Synaptotagmin-1 and -7 are functionally overlapping $\mathrm{Ca} 2+$ sensors for exocytosis in adrenal chromaffin cells. Proc Natl Acad Sci USA 105:3998-4003.

Scoville WB, Milner B (1957) Loss of recent memory after bilateral hippocampal lesions. J Neurol Neurosurg Psychiatry 20:11-21.

Sederberg PB, Kahana MJ, Howard MW, Donner EJ, Madsen JR (2003) Theta and gamma oscillations during encoding predict subsequent recall. J Neurosci 23:10809-10814.

Segovia M, Ales E, Montes MA, Bonifas I, Jemal I, Lindau M, Maximov A, Sudhof TC, de Toledo GA (2010) Push-and-pull regulation of the fusion pore by synaptotagmin-7. Proc Natl Acad Sci USA 107:19032-19037.

Senn W, Segev I, Tsodyks M (1998) Reading neuronal synchrony with depressing synapses. Neural Comput 10:815-819.

Sharp, P (2006) Subicular place cells generate the same 'map' for different environments: comparison with hippocampal cells. Behav Brain Res 174:206-214.

Sheng ZH, Rettig J, Cook T, Catterall WA (1996) Calcium-dependent interaction of Ntype calcium channels with the synaptic core complex. Nature 379:451-454.

Shimamura AP (2003) Neural basis of memory: systems level. In: Encyclopedia of cognitive science (Nadel L, ed), pp218-224. London: Macmillan.

Shin OH, Xu J, Rizo J, Sudhof TC (2009) Differential but convergent functions of $\mathrm{Ca} 2+$ binding to synaptotagmin-1 C2 domains mediate neurotransmitter release. Proc Natl Acad Sci USA 106:16469-16474.

Siegelbaum SA, Kandel ER (1991) Learning-related synaptic plasticity: LTP and LTD. Curr Opin Neurobiol 1:113-120. 
Siekmeier PJ, Hasselmo ME, Howard MW, Coyle J (2007) Modeling of contextdependent retrieval in hippocampal region CA1: implications for cognitive function in schizophrenia. Schizophr Res 89:177-190.

Silberberg G, Wu C, Markram H (2004) Synaptic dynamics control the timing of neuronal excitation in the activated neocortical microcircuit. J Physiol 556:19-27.

da Silva BC, Basso EW, Bazzan ALC, Engel PM (2006) Dealing with non-stationary environments using context detection. Proc 23rd Int Conf Machine Learn: 217-224.

Simons SB, Escobedo R, Yasuda R, Dudek SM (2009) Regional differences in hippocampal calcium handling provide a cellular mechanism for limiting plasticity. Proc Natl Acad Sci USA 106:14080-14084.

Sinha SR, Wu LG, Saggau P (1997) Presynaptic calcium dynamics and transmitter release evoked by single action potentials at mammalian central synapses. Biophys $\mathbf{J}$ 72:637-651.

Siri B, Berry H, Cessac B, Delord B, Quoy M (2008) A mathematical analysis of the effects of Hebbian learning rules on the dynamics and structure of discrete-time random recurrent neural networks. arXiv:0705.3690v2.

Smith DM, Mizumori SJY (2006) Learning-related development of context-specific neuronal responses to places and events: the hippocampal role in context processing. $\mathrm{J}$ Neurosci 26:3154-3163.

Solstad T, Boccara CN, Kropf E, Moser MB, Moser EI (2008) Representation of geometric borders in the entorhinal cortex. Science 322:1865-1868.

Song D, Marmarellis VZ, Berger TW (2009) Parametric and non-parametric modeling of short-term synaptic plasticity. Part I: computational study. J Comput Neurosci 26:1-19.

Speed HE, Dobrunz LE (2009) Developmental changes in short-term facilitation are opposite at temporoammonic synapses compared to Schaffer collateral synapses onto CA1 pyramidal cells. Hippocampus 19:187-204.

Stanley EF (1997) The calcium channel and the organization of the presynaptic transmit ter release face. Trends Neurosci 20:404-409.

Stark C (2007) Functional role of the human hippocampus. In: The hippocampus book (Anderson P, Morris R, Amaral D, Bliss T, O'Keefe J, eds), pp549-580. Oxford: Oxford UP.

Staubli U, Lynch G (1987) Stable hippocampal long-term potentiation elicited by 'theta' pattern stimulation. Brain Res 435:227-234.

Stella F, Cerasti E, Si B, Jezek K, Treves A (2011) Self-organization of multiple spa- 
tial and context memories in the hippocampus. Neurosci Biobehav Rev: epub ahead of print.

Stevens CF (2003) Neurotransmitter release at central synapses. Neuron 40:381-388.

Stewart BA, Mohtashami M, Trimble WS, Boulianne GL (2000) SNARE proteins contribute to calcium cooperativity of synaptic transmission. Proc Natl Acad Sci USA 97:13955-13960.

Stief F, Zuschratter W, Hartmann K, Schmitz D, Draguhn A (2007) Enhanced synaptic excitation-inhibition ratio in hippocampal interneurons of rats with temporal lobe epilepsy. Eur J Neurosci 25:519-528.

Strong SP, Koberle R, van Steveninck RR, Bialek W (1998) Entropy and information in neural spike trains. Phys Rev Lett 80:197-200.

Sudhof TC (2004) The synaptic vesicle cycle. Annu Rev Neurosci 27:509-547.

Sudhof TC, Rizo J (2011) Synaptic vesicle exocytosis. Cold Spring Harb Perspect Biol 3:a005637.

Sugita S, Han W, Butz S, Liu X, Fernandez-Chacon R, Lao Y, Sudhof TC (2001) Synaptotagmin VII as a plasma membrane $\mathrm{Ca} 2+$ sensor in exocytosis. Neuron 30:459-473.

Sugita S, Shin OH, Han W, Lao Y, Sudhof TC (2010) Synaptotagmins form a hierarchy of exocytotic $\mathrm{Ca} 2+$ sensors with distinct $\mathrm{Ca} 2+$ affinities. EMBO J 21:270-280.

Suh J, Rivest AJ, Nakashiba T, Tominaga T, Tonegawa S (2011) Entorhinal cortex layer III input to the hippocampus is crucial for temporal association memory. Science 334:1415-1420.

Summerfield C, Behrens TE, Koechlin E (2011) Perceptual classification in a rapidly changing environment. Neuron 71:725-736.

Sun HY, Lyons SA, Dobrunz LE (2005) Mechanisms of target-cell specific short-term plasticity at Schaffer collateral synapses onto interneurones versus pyramidal cells in juvenile rats. J Physiol 568:815-830.

Sun J, Pang ZP, Qin D, Fahim AT, Adachi R, Sudhof TC (2007) A dual-Ca2+-sensor model for neurotransmitter release in a central synapse. Nature 450:676-682.

Suthana N, Haneef Z, Stern J, Mukamel R, Behnke E, Knowlton B, Fried I (2012) Memory enhancement and deep-brain stimulation of the entorhinal area. N Engl J Med 366:502-510.

Sweatt JD (2004) Hippocampal function in cognition. Psychpharmacology 174:99-110.

Szirtes G, Poczos B, Lorincz A (2004) Neural Kalman filter. Neurocomputing 
65-66:349-355.

Szucs A (1998) Applications of spike density function analysis of neuronal firing patterns. J Neurosci Methods 81:159-167.

Tahvildari B, Fransen E, Alonson AA, Hasselmo ME (2007) Switching between "on" and "off" states of persistent activity in lateral entorhinal layer III neurons. Hippocampus 17:257-263.

Takahashi H, Magee JC (2009) Pathway interactions and synaptic plasticity in the dendritic tuft regions of CA1 pyramidal neurons. Neuron 62:102-111.

Tamamaki N, Nojyo Y (1995) Preservation of topography in the connections between the subiculum, field CA1, and the entorhinal cortex in rats. J Comp Neurol 353:379-390.

Tang J, Maximov A, Shin OH, Dai H, Rizo J, Sudhof TC (2006) A complexin/synaptotagmin 1 switch controls fast synaptic vesicle exocytosis. Cell 126:1175-1187.

Tank DW, Regehr WG, Delaney KR (1995) A quantitative analysis of presynaptic calcium dynamics that contribute to short-term enhancement. J Neurosci 15:7940-7952.

Taube JS, Muller RU, Ranck JB Jr (1990) Head-direction cells recorded from the postsubiculum in freely moving rats. II. Effects of environmental manipulations. J Neurosci 10:436-447.

Thurley K, Liebold C, Gundlefinger A, Schmitz D, Kempter R (2008) Phase precession through synapse facilitation. Neural Comput 20:1285-1324.

Tolman EC (1948) Cognitive maps in rats and men. Psychol Rev 55:189-208.

Torres JJ, Marro J, Garrido PL, Cortes JM, Ramos F, Munoz MA (2005) Effects of static and dynamic disorder on the performance of neural automata. Biophys Chem 115:285-288.

Torres JJ, Cortes JM, Marro J, Kappan HJ (2007) Attractor neural networks with activity-dependent synapses: the role of synaptic facilitation. Neurocomputing 70:2022-2025.

Tort ABL, Komorowski RW, Manns JR, Kopell NJ, Eichenbaum H (2009) Thetagamma coupling increases during the learning of item-context association. Proc Natl Acad Sci USA 106:20942-20947.

Traub RD, Wong RKS, Miles R, Michelson H (1991) A model of a CA3 hippocampal pyramidal neuron incorporating voltage-clamp data on intrinsic conductances. J Neurophysiol 66:635-650.

Treves A (2004) Computational constraints between retrieving the past and predicting the future, and the CA3-CA1 differentiation. Hippocampus 14:539-556. 
Treves A (2009) Spatial cognition, memory capacity, and the evolution of mammalian hippocampal networks. In: Cognitive biology: evolutionary and developmental perspectives on mind, brain, and behavior (Tommasi L, Peterson MA, Nadel L, eds), pp41-59. Cambridge MA: The MIT Press.

Trommershauser J, Schneggenburger R, Zippelius A, Neher E (2003) Heterogeneous presynaptic release probabilities: functional relevance for short-term plasticity. Biophys J 84:1563-1579.

Trussell LO, Zhang S, Raman IM (1993) Desensitization of AMPA receptors upon multiquantal neurotransmitter release. Neuron 10:1185-1196.

Tse D, Langston RF, Kakeyama M, Bethus I, Spooner PA, Wood ER, Witter MP, Morris RGM (2007) Schemas and memory consolidation. Science 316:76-82.

Tsodyks M, Markram H (1997) The neural code between neocortical pyramidal neurons depends on neurotransmitter release proability. Proc Natl Acad Sci USA 94:719-723.

Tsodyks M, Pawelzik K, Markram H (1998) Neural networks with dynamics synapses. Neural Comput 10:821-835.

Tsukada M, Fukushima Y (2010) A context-sensitive mechanism in hippocampal CA1 networks. Bull Math Biol 73:417-435.

Tulving E (2002) Episodic memory: from mind to brain. Annu Rev Psychol 53:1-25.

Uhlenbeck GE, Ornstein LS (1930) On the theory of the Brownian motion. Phys Rev 36:823-841.

Uhlhaas PJ, Singer W (2010) Abnormal neural oscillations and synchrony in schizophre nia. Nat Rev Neurosci 11:100-113.

Urban NN, Henze DA, Barrionuevo G (2001) Revisiting the role of the hippocampal mossy fiber synapse. Hippocampus 11:408-417.

Vago DR, Bevan A, Kesner RP (2007) The role of the direct perforant path input to the CA1 subregion of the dorsal hippocampus in memory retention and retrieval. Hippocam pus 17:977-987.

Vann SD, Albasser MM (2011) Hippocampus and neocortex: recognition and spatial memory. Curr Opin Neurobiol 21:440-445.

Vapnik VN (1999) An overview of statistical learning theory. IEEE Trans Neural Netw 10:988-999.

Varela JA, Sen K, Gibson J, Fost J, Abbot LF, Nelson SB (1997) A quantitative descrip. tion of short-term plasticity at excitatory synapses in layer $2 / 3$ of rat primary visual 
cortex. J Neurosci 17:7926-7940.

Vertes RP, Hoover WB, Szigeti-Buck K, Leranth C (2007) Nucleus reuniens of the midline thalamus: link between the medial prefrontal cortex and the hippocampus. Brain Res Bull 71:601-609.

Victor JD, Purpura KP (1997) Metric-space analysis of spike trains: theory, algorithms and application. Network 8:127-164.

Villarreal DM, Gross AL, Derrick BE (2007) Modulation of CA3 afferent inputs by novelty and theta rhythm. J Neurosci 27:13547-13467.

Villette V, Poindessous-Jazat F, Simon A, Lena C, Roullot E, Bellessort B, Epelbaum J, Dutar P, Stephan A (2010) Decreased rhythmic GABAergic septal activity and memory-associated $\theta$ oscillations after hippocampal amyloid- $\beta$ pathology in the rat. $\mathbf{J}$ Neurosci 30:10991-11003.

Vinogradova OS (1984) Functional organization of the limbic system in the process of registration of information: facts and hypotheses. In: The hippocampus (Isaakson RL, Pribram KH, eds), pp1-69. New York: Plenum Press.

Vinogradova OS (2001) Hippocampus as comparator: role of the two input and two output systems of the hippocampus in selection and registration of information. Hippocampus 11:578-598.

Vinogradova OS, Semyonova TP, Konovalov VP (1970) Trace phenomena in the neurons of the limbic structures. In: Biology of memory (Pribram K, Broadbent A, eds), pp191-221. New York: Academic Press.

Volman V, Levine H, Ben-Jacob E, Sejnowski TJ (2009) Locally balanced dendritic integration by short-term synaptic plasticity and active dendritic conductances. J Neurophysiol 102:3234-3250.

Walter AM, Groffen AJ, Sorensen JB, Verhage M (2011) Multiple Ca2+ sensors in secretion: teammates, competitors or autocrats? Trends Neurosci 34:487-497.

Wang SH, Morris RGM (2010) Hippocampal-neocortical interactions in memory formation, consolidation, and reconsolidation. Annu Rev Psychol 61:49-79.

Wen H, Linhoff MW, McGinley MJ, Li GL, Corson GM, Mandel G (2010) Distinct roles for two synaptotagmin isoforms in synchronous and asynchronous transmitter release at zebrafish neuromuscular junction. Proc Natl Acad Sci USA June 16, 2010 Early Edition.

Whishaw IQ, Wallace DG (2003) On the origins of autobiographical memory. Behav Brain Res 138:113-119.

Whitlock JR, Heynen AJ, Shuler MG, Bear MF (2006) Learning induces long-term 
potentiation in the hippocampus. Science 313:1093-1097.

Wiebe SP, Staubli UV (1999) Dynamic filtering of recognition memory codes in the hippocampus. J Neurosci 19:10562-10574.

Wikenheiser AM, Redish AD (2012) Hippocampal sequences link past, present, and future. Trends Cog Sci 16:361-362.

Williamson A, Spencer DD (1994) Electrophysiological characterization of CA2 pyramidal cells from epileptic humans. Hippocampus 4:226-237.

Willmore B, Tolhurst DJ (2001) Characterizing the sparseness of neural codes. Network: Comput Neural Syst 12:225-279.

Wills TJ, Lever C, Cacucci F, Burgess N, O'Keefe J (2005) Attractor dynamics in the hippocampal representation of the local environment. Science 308:873-876.

Wilson IA, Gallagher M, Eichenbaum H, Tanila H (2006) Neurocognitive aging: prior memories hinder new hippocampal encoding. Trends Neurosci 29:662-670.

Wilson MA, McNaughton BL (1993) Dynamics of the hippocampal ensemble code for space. Science 261:1055-1058.

Wilson RI, Nicoll RA (2002) Endocannabinoid signaling in the brain. Science 296:678-682.

Wolfel M, Lou X, Schneggenburger R (2007) A mechanism intrinsic to the vesicle fusion machinery determines fast and slow transmitter release at a large CNS synapse. J Neurosci 27:3198-3210.

Wood ER, Dudchenko PA, Eichenbaum H (1999) The global record of memory in hippocampal neuronal activity. Nature 397:613-616.

Wu ZZ, Li DP, Chen SR, Pan HL (2009) Aminopyridines potentiate synaptic and neuromuscular transmission by targeting the voltage-activated calcium channel subunit. J Biol Chem 284:36453-36461.

Xu J, Clancy CE (2008) Ionic mechanisms of endogenous bursting in CA3 hippocampal pyramidal neurons: a model study. PLoS ONE 3:e2056.

Xu W, Morishita W, Buckmaster PS, Pang ZP, Malenka RC, Sudhof TC (2012) Distinct neuronal coding schemes in memory revealed by selective erasure of fast synchronous synaptic transmission. Neuron 73:990-1001.

Xu-Friedman M, Regehr W (2004) Structural contributions to short-term synaptic plasticity. Physiol Rev 84:69-85.

Xue M, Craig KC, Shin OH, Li L, Brautigam CA, Tomchick DR, Sudhof TC, Rosenmund C, Rizo J (2010) Structural and mutational analysis of functional differentiation 
between synaptotagmins-1 and -7. PLoS ONE 5:e12544.

Yamaguchi Y, Sato N, Wagatsuma H, Wu Z, Molter C, Aota Y (2007) A unified view of theta-phase coding in the entorhinal-hippocampal system. Curr Opin Neurobiol 17:197-204.

Yang X, Kaeser-Woo YJ, Pang ZP, Xu W, Sudhof TC (2010) Complexin clamps asynchronous release by blocking a secondary $\mathrm{Ca} 2+$-sensor via its accessory $\alpha$-helix. Neuron 68:907-920.

Yao CK, Lin YQ, Ly CV, Ohyama T, Haueter CM, Moiseenkova-Bell VY, Wensel TG, Bellen HJ (2009) A synaptic vesicle-associated Ca2+ channel promotes endocytosis and couples exocytosis to endocytosis. Cell 138:947-960.

Yassa MA, Stark CEL (2011) Pattern seperation in the hippocampus. Trends Neurosci 34:515-525.

Yoshihara M, Littleton T (2002) Synaptotagmin I functions as a calcium sensor to synchronize neurotransmitter release. Neuron 36:897-908.

Young SM Jr, Neher E (2009) Synaptotagmin has an essential function in synaptic vesicle positioning for synchronous release in addition to its role as a calcium sensor. Neuron 63:482-496.

Zador AM (2001) Synaptic connectivity and computation. Nat Neurosci 4:1157-1158.

Zador AM, Dobrunz LE (1997) Dynamic synapses in the cortex. Neuron 19:1-4.

Zhang Z, Hui E, Chapman ER, Jackson MB (2010) Regulation of exocytosis and fusion pores by synaptotagmin-effector interactions. Mol Biol Cell 21:2821-2831.

Zhao C, Deng W, Gage FH (2008) Mechanisms and functional implications of adult neurogenesis. Cell 132:645-660.

Zucker RS, Regehr WG (2002) Short-term synaptic plasticity. Annu Rev Physiol 64:355-405.

Zucker RS, Kullmann DM, Bennett M (1999) Release of neurotransmitters. In: Fundamental neuroscience (Zigmond MJ, Bloom FL, Landis SC, Roberts JL, Squire LR, eds), pp155-192. San Diego: Academic Press. 


\section{Appendices}


A P P E N D I X A

\section{DSCI model equations}

\section{Terms}

Table A.1. DSCI model parameters. Color code: Blue $\rightarrow$ calcium processes. Green $\rightarrow$ vesicle pools and refilling. Pink $\rightarrow$ vesicle release (exocytosis) processes.

\begin{tabular}{|c|c|}
\hline Parameter & Description \\
\hline$\left[\mathrm{Ca}^{2+}\right]_{\text {res }, 0}$ & Initial residual calcium concentration \\
\hline$\overline{\Delta_{\mathrm{Ca}, \mu}}$ & Peak nanodomain calcium concentration \\
\hline$\Delta_{\text {Ca,int }}$ & Residual calcium update, intermediate component \\
\hline$\Delta_{\text {Ca,slow }}$ & Residual calcium update, slow component \\
\hline$\tau_{\mathrm{Ca}, \text { int }}$ & Calcium decay time constant, intermediate component \\
\hline$\tau_{\text {Ca,slow }}$ & Calcium decay time constant, slow component \\
\hline$n_{\text {rest }}$ & Number of vesicles at rest \\
\hline$\phi_{n}$ & Fraction of total vesicles in willing pool \\
\hline$\tau_{\text {fast }}$ & Willing pool reloading rate time constant \\
\hline$\tau_{\text {slow }}$ & Reluctant pool reloading rate time constant \\
\hline$\tau_{k, \text { refill }}$ & Willing pool accelerated reloading decay time constant \\
\hline Amp $_{\text {refill,fast }}$ & Willing pool accelerated reloading amplitude \\
\hline Kd $_{\text {refill,fast }}$ & Willing pool accelerated reloading dissociation constant \\
\hline$\phi_{\mathrm{Ca}, \mu}$ & Fraction of nanodomain calcium concentration at reluctant vesicles \\
\hline$p_{\text {syt1,SNARE,min,willing }}$ & Min probability of syt1 binding to SNARE at willing vesicles \\
\hline$p_{\text {syt } 1, \text { SNARE,min,reluctant }}$ & Min probability of syt1 binding to SNARE at reluctant vesicles \\
\hline$p_{\text {syt7,SNARE,min }}$ & Min probability of syt7 binding to SNARE at all vesicles \\
\hline$p_{\text {syt1,SNARE,max,willing }}$ & Max probability of syt1 binding to SNARE at willing vesicles \\
\hline$p_{\text {syt1,SNARE,max,reluctant }}$ & Max probability of syt1 binding to SNARE at reluctant vesicles \\
\hline$p_{\text {syt7,SNARE,max }}$ & Max probability of syt7 binding to SNARE at all vesicles \\
\hline $\mathbf{K d}_{\text {Ca,syt1,SNARE }}$ & Calcium concentration at half-max syt 1 -to-SNARE binding \\
\hline Kd $_{\text {Ca,syt7,SNARE }}$ & Calcium concentration at half-max syt7-to-SNARE binding \\
\hline $\mathbf{K d}_{\text {Ca,syt1,trigger }}$ & Calcium concentration at half-max syt 1 -triggered fusion \\
\hline $\mathbf{K d}_{\text {Ca,syt } 7 \text {,rigger }}$ & Calcium concentration at half - max syt 7 -triggered fusion \\
\hline$E_{\text {syt1 }}$ & Syt1 energetic contribution to membrane fusion \\
\hline$E_{\text {syt7 }}$ & Syt7 energetic contribution to membrane fusion \\
\hline$E_{\text {fusion }}$ & Energetic barrier to membrane fusion \\
\hline$N_{\text {SNARE }}$ & Number of SNAREs associated with each vesicle \\
\hline$n_{\text {Ca,syt1,trigger }}$ & Cooperativity of syt1-triggered fusion in calcium \\
\hline$n_{\text {Ca,syt7,trigger }}$ & Cooperativity of syt7-triggered fusion in calcium \\
\hline$n_{\text {Ca,syt1,SNARE }}$ & Cooperativity of syt1-to-SNARE binding in calcium \\
\hline$n_{\text {Ca,syt7,SNARE }}$ & Cooperativity of syt7-to-SNARE binding in calcium \\
\hline
\end{tabular}




\section{Equations}

Mean resting vesicle pool populations (willing and reluctant).

$$
\begin{gathered}
n_{\text {rest,willing }}=n_{\text {rest }} \phi_{n}=n_{\text {willing }, 0} \\
n_{\text {rest,reluctant }}=n_{\text {rest }}\left(1-\phi_{n}\right)=n_{\text {reluctant }, 0}
\end{gathered}
$$

Calcium dynamics ( $M$ is the number of spikes in the input spike train).

$$
\begin{gathered}
\frac{d}{d t}\left[\mathrm{Ca}^{2+}\right]_{\text {res,int }}(t)=-\frac{\left[\mathrm{Ca}^{2+}\right]_{\text {res,int }}(t)}{\tau_{\mathrm{Ca}, \text { int }}}+\Delta_{\mathrm{Ca}, \text { int }} \delta\left(t-t_{i}\right), \forall i \in\{1, \ldots, M\} \\
\frac{d}{d t}\left[\mathrm{Ca}^{2+}\right]_{\text {res,slow }}(t)=-\frac{\left[\mathrm{Ca}^{2+}\right]_{\text {res,int }}(t)}{\tau_{\mathrm{Ca}, \text { slow }}}+\Delta_{\mathrm{Ca}, \text { slow }} \delta\left(t-t_{i}\right), \forall i \in\{1, \ldots, M\} \\
{\left[\mathrm{Ca}^{2+}\right]_{\mathrm{res}}(t)=\left[\mathrm{Ca}^{2+}\right]_{\mathrm{res}, 0}+\left[\mathrm{Ca}^{2+}\right]_{\mathrm{res}, \text { int }}(t)+\left[\mathrm{Ca}^{2+}\right]_{\mathrm{res}, \text { slow }}(t)} \\
{\left[\mathrm{Ca}^{2+}\right]_{\mu, i}=\left[\mathrm{Ca}^{2+}\right]_{\mathrm{res}}\left(t_{i}\right)+\Delta_{\mathrm{Ca}, \mu}}
\end{gathered}
$$

Vesicle pool refilling.

$$
\begin{aligned}
& n_{\text {willing }}\left(t_{i}\right)=n_{\text {willing }}\left(t_{i-1}\right)+n_{\text {refill,willing }}\left(t_{i}\right), \forall i \in\{1, \ldots, M\} \\
& n_{\text {reluctant }}\left(t_{i}\right)=n_{\text {reluctant }}\left(t_{i-1}\right)+n_{\text {refill,reluctant }}\left(t_{i}\right), \forall i \in\{1, \ldots, M\} \\
& n_{\text {refill,willing }}\left(t_{i}\right)=\min \left(n_{\text {rest,willing }}-n_{\text {willing }}\left(t_{i}\right), n_{\text {rest,willing }} \eta_{\text {willing }}\left(t_{i}\right)\right) \\
& n_{\text {refill,reluctant }}\left(t_{i}\right)=\min \left(n_{\text {rest,reluctant }}-n_{\text {reluctant }}\left(t_{i}\right), n_{\text {rest,reluctant }} \eta_{\text {reluctant }}\left(t_{i}\right)\right) \\
& \frac{d \eta_{\text {reluctant }}(t)}{d t}=\frac{1-\eta_{\text {reluctant }}(t)}{\tau_{\text {slow }}}, \eta_{\text {reluctant }}(0)=0 \\
& \eta_{\text {willing }}(t)=\frac{k_{\text {refill,fast }}(t) \tilde{\eta}_{\text {willing }}(t)+\eta_{\text {reluctant }}(t)}{k_{\text {refill,fast }}(t)+1} \\
& \frac{d \tilde{\eta}_{\text {willing }}(t)}{d t}=\frac{1-\tilde{\eta}_{\text {willing }}(t)}{\tau_{\text {fast }}} \\
& k_{\text {refill,fast }}(t)=\operatorname{Amp}_{\text {refill,fast }} \tilde{k}(t)\left(\frac{\left[\mathrm{Ca}^{2+}\right]_{\text {res }}(t)}{\left[\mathrm{Ca}^{2+}\right]_{\text {res }}(t)+\mathrm{Kd}_{\text {refill,fast }}}\right)
\end{aligned}
$$




$$
\frac{d \tilde{k}(t)}{d t}=-\frac{\tilde{k}(t)}{\tau_{k, \text { refill }}}
$$

Exocytosis.

$$
\begin{aligned}
& P_{\text {release }}\left(t_{i}\right)=1-\left(1-P_{\text {ves,willing }}\left(t_{i}\right)\right)^{n_{\text {willing }}\left(t_{i}\right)}\left(1-P_{\text {ves,reluctant }}\left(t_{i}\right)\right)^{n_{\text {reluctant }}\left(t_{i}\right)} \\
& P_{\mathrm{ves}, \text { willing }, i}=\frac{e^{-E_{\mathrm{diff}, \text { willing, }, i}}}{1+e^{-E_{\mathrm{diff}, \text { willing, }, i}}} \\
& P_{\mathrm{ves}, \text { reluctant }, i}=\frac{e^{-E_{\mathrm{diff}, \text { elluctant }, i}}}{1+e^{-E_{\mathrm{diff}, \text { eluctant }, i}}} \\
& E_{\text {diff,willing }, i}=E_{\mathrm{SNARE}, \text { willing }, i}-E_{\text {fusion }} \\
& E_{\text {diff, reluctant }, i}=E_{\mathrm{SNARE}, \text { reluctant }, i}-E_{\text {fusion }} \\
& E_{\mathrm{SNARE}, \text { willing }, i}=E_{\mathrm{syt} 1} \hat{N}_{\mathrm{syt} 1, \text { willing }, i}+E_{\mathrm{syt}} \hat{N}_{\mathrm{syt} 7, \text { willing }, i} \\
& E_{\mathrm{SNARE}, \text { reluctant }, i}=E_{\mathrm{syt1}} \hat{N}_{\text {syt } 1, \text { reluctant }, i}+E_{\mathrm{syt} 7} \hat{N}_{\text {syt7, reluctant }, i} \\
& \hat{N}_{\text {syt1,willing }, i}=N_{\text {syt1,willing }}\left(t_{i}\right) \Phi_{\text {syt1, willing }, i} \\
& \hat{N}_{\text {syt7,willing }, i}=N_{\text {syt7,willing }}\left(t_{i}\right) \Phi_{\text {syt7,willing }, i} \\
& \hat{N}_{\text {syt 1, reluctant }, i}=N_{\text {syt } 1, \text { reluctant }}\left(t_{i}\right) \Phi_{\text {syt } 1, \text { reluctant }, i} \\
& \hat{N}_{\text {syt } 7, \text { reluctant }, i}=N_{\text {syt } 7 \text {,reluctant }}\left(t_{i}\right) \Phi_{\text {syt } 7, \text { reluctant }, i}
\end{aligned}
$$

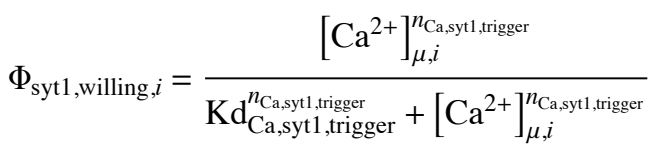

$$
\begin{aligned}
& \Phi_{\text {syt7,willing }, i}=\frac{\left[\mathrm{Ca}^{2+}\right]_{\mu, i}^{n_{\text {Ca,sy7,trigger }}}}{\mathrm{Kd}_{\text {Ca,syt7, trigger }}^{n_{\text {Ca,yst,tigger }}}+\left[\mathrm{Ca}^{2+}\right]_{\mu, i}^{n_{\text {Ca,sy7, trigger }}}}
\end{aligned}
$$

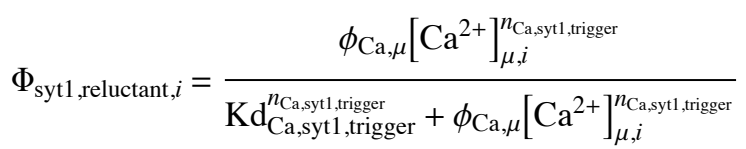




$$
\begin{aligned}
& \Phi_{\text {syt7,reluctant }, i}=\frac{\phi_{\mathrm{Ca}, \mu}\left[\mathrm{Ca}^{2+}\right]_{\mu, i}^{n_{\text {Ca,syt7,trigger }}}}{\mathrm{Kd}_{\mathrm{Ca}, \text { syt7,trigger }}^{n_{\text {Ca,yyt,tigger }}}+\phi_{\mathrm{Ca}, \mu}\left[\mathrm{Ca}^{2+}\right]_{\mu, i}^{n_{\mathrm{Ca}, \text { yt7, trigger }}}} \\
& N_{\text {syt } 1, \text { willing }}\left(t_{i}\right)=N_{\text {SNARE }} \hat{p}_{\text {syt } 1, \text { SNARE,willing }}\left(t_{i}\right) \\
& N_{\text {syt7,willing }}\left(t_{i}\right)=N_{\text {SNARE }} \hat{p}_{\text {syt7,SNARE,willing }}\left(t_{i}\right) \\
& N_{\text {syt } 1, \text { reluctant }}\left(t_{i}\right)=N_{\text {SNARE }} \hat{p}_{\text {syt } 1, \text { SNARE,reluctant }}\left(t_{i}\right) \\
& N_{\text {syt7,reluctant }}\left(t_{i}\right)=N_{\text {SNARE }} \hat{p}_{\text {syt7,SNARE,reluctant }}\left(t_{i}\right) \\
& \hat{p}_{\text {syt1,SNARE,willing }}\left(t_{i}\right)=p_{\text {syt1,SNARE,willing }}\left(t_{i}\right)-p_{\text {comp,willing }}\left(t_{i}\right)+p_{\text {syt1,comp,willing }}\left(t_{i}\right) \\
& \hat{p}_{\text {syt7,SNARE,willing }}\left(t_{i}\right)=p_{\text {syt7,SNARE,willing }}\left(t_{i}\right)-p_{\text {comp,willing }}\left(t_{i}\right)+p_{\text {syt7,comp,willing }}\left(t_{i}\right) \\
& \hat{p}_{\text {syt1,SNARE,reluctant }}\left(t_{i}\right)=p_{\text {syt } 1, \text { SNARE,reluctant }}\left(t_{i}\right)-p_{\text {comp,reluctant }}\left(t_{i}\right)+p_{\text {syt } 1, \text { comp,reluctant }}\left(t_{i}\right) \\
& \hat{p}_{\text {syt7,SNARE,reluctant }}\left(t_{i}\right)=p_{\text {syt7,SNARE,reluctant }}\left(t_{i}\right)-p_{\text {comp,reluctant }}\left(t_{i}\right)+p_{\text {syt7,comp,reluctant }}\left(t_{i}\right) \\
& p_{\text {syt } 1, \text { comp,willing }}\left(t_{i}\right)=\frac{p_{\text {comp,willing }}\left(t_{i}\right)}{\frac{p_{\text {syt }, \text { SNARE }}\left(t_{i}\right)}{p_{\text {syt } 1, \text { NARE,willing }}\left(t_{i}\right)}+1} \\
& p_{\text {syt7,comp,willing }}\left(t_{i}\right)=p_{\text {comp,willing }}\left(t_{i}\right)-p_{\text {syt } 1, \text { comp,willing }}\left(t_{i}\right) \\
& p_{\text {syt } 1, \text { comp,reluctant }}\left(t_{i}\right)=\frac{p_{\text {comp,reluctant }}\left(t_{i}\right)}{\frac{p_{\text {syt }, \mathrm{SNARE}}\left(t_{i}\right)}{p_{\text {syt } 1, \mathrm{SNARE}, \text { reluctant }}\left(t_{i}\right)}+1} \\
& p_{\text {syt7,comp,reluctant }}\left(t_{i}\right)=p_{\text {comp,reluctant }}\left(t_{i}\right)-p_{\text {syt7,comp,reluctant }}\left(t_{i}\right) \\
& p_{\text {comp,willing }}\left(t_{i}\right)=p_{\text {syt } 1, \mathrm{SNARE}, \text { willing }}\left(t_{i}\right) p_{\text {syt7,SNARE }}\left(t_{i}\right) \\
& p_{\text {comp,reluctant }}\left(t_{i}\right)=p_{\text {syt } 1, \text { SNARE,reluctant }}\left(t_{i}\right) p_{\text {syt7,SNARE }}\left(t_{i}\right) \\
& \frac{d}{d t} p_{\text {syt } 1, \text { SNARE,willing }}(t)= \\
& \frac{\left(p_{\text {syt } 1, \text { SNARE,max,willing }}-p_{\text {syt1,SNARE,min,willing }}\right)}{N_{\text {SNARE }}} \frac{d}{d t} \operatorname{Syt}_{\text {CaSNARE }} \text { willing }(t)
\end{aligned}
$$




$$
\begin{aligned}
& \frac{d}{d t} p_{\text {syt } 1, \text { SNARE,reluctant }}(t)= \\
& \frac{1}{N_{\text {SNARE }}}\left(p_{\text {syt } 1, \text { SNARE,max,reluctant }}-p_{\text {syt } 1, \mathrm{SNARE}, \text { min,reluctant }}\right) \frac{d}{d t} \operatorname{Syt}_{1 \mathrm{CaSNARE}} \mathrm{reluctant}(t) \\
& \frac{d}{d t} p_{\text {syt7,SNARE }}(t)=\frac{\left(p_{\text {syt } 7, \text { SNARE,max }}-p_{\text {syt7,SNARE,min }}\right)}{N_{\text {SNARE }}} \frac{d}{d t} \operatorname{Syt7CaSNARE~}(t) \\
& \frac{d}{d t} \operatorname{Syt}_{t} \operatorname{CaSNARE}_{\text {willing }}(t)=
\end{aligned}
$$

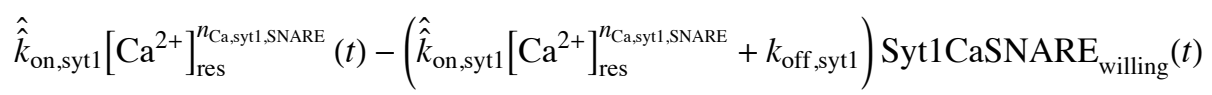

$$
\begin{aligned}
& \frac{d}{d t} \operatorname{Syt}_{\mathrm{CaSNARE}} \mathrm{reuctant}(t)=
\end{aligned}
$$

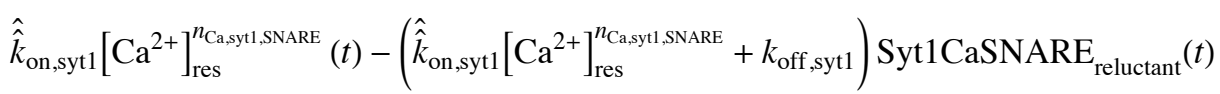

$$
\begin{aligned}
& \frac{d}{d t} \operatorname{Syt} 7 \operatorname{CaSNARE}(t)= \\
& \hat{\hat{k}}_{\text {on, syt } 7}\left[\mathrm{Ca}^{2+}\right]_{\text {res }}^{n_{\text {Casyt } 7, \text { NARE }}}(t)-\left(\hat{\hat{k}}_{\text {on, syt } 7}\left[\mathrm{Ca}^{2+}\right]_{\text {res }}^{n_{\text {Casyt } 7, \text { SARE }}}+k_{\text {off, syt7 }}\right) \operatorname{Syt7CaSNARE}(t) \\
& \hat{\hat{k}}_{\text {on,syt1 }}=\hat{k}_{\text {on,syt1 }} N_{\text {SNARE }} \\
& \hat{k}_{\text {on,syt } 1}=k_{\text {on,syt } 1}[\text { Syt } 1]
\end{aligned}
$$

The code to run the DSCI model was implemented in Matlab. 


\section{A P P E N D I X B}

\section{The Hill equation}

Cooperative dose-response curves can be approximated by the Hill equation, which describes the fraction of macromolecules saturated by a ligand as a function of ligand concentration at equilibrium. $K_{A}$ is the ligand concentration producing half occupation, and is related to the dissociation constant, $K_{d}$, by, $K_{d}=K_{A}{ }^{n}$, where $n$ is the cooperativity, interpreted as the number of ligands needed for full binding.

The kinetics of a chemical or molecular reaction process in which two compounds, $A$ and $B$, react to form a third compund, $C$, is written as,

$$
A+B \underset{k_{\mathrm{off}}}{\stackrel{k_{\mathrm{on}}}{\rightleftharpoons}} A B
$$

where $k_{\text {on }}$ and $k_{\text {off }}$ are the forward and reverse reaction rate constants. In a biomolecular reaction, $B$ could be a protein, and $A$, a ligand. The expression is shorthand for the equations,

$$
\begin{aligned}
& \frac{d[B]}{d t}=-k_{\mathrm{on}}[A][B]+k_{\mathrm{off}}[B] \\
& \frac{d[A]}{d t}=-k_{\mathrm{on}}[A][B]+k_{\mathrm{off}}[A] \\
& \frac{d[A B]}{d t}=k_{\mathrm{on}}[A][B]-k_{\mathrm{off}}[A B]
\end{aligned}
$$

The reaction is in quilibrium when the formation $=k_{\text {on }}[A][B]$, and the breakdown $=k_{\text {off }}[A B]$, (i.e, the forward and reverse rates) are the same (the Law of Mass Action):

$$
\begin{gathered}
\frac{d[A B]}{d t}=k_{\text {on }}[A][B]-k_{\text {off }}[A B]=0 \\
{[A][B] k_{\text {on }}=[A B] k_{\text {off }}}
\end{gathered}
$$

The dissociation constant, $K_{D}$, is defined as the ratio of the forward and reverse rate constants, and is 
equivalent to the inverse of the association constant, $K_{A}$ :

$$
K_{D}=\frac{1}{K_{A}}=\frac{k_{\text {on }}}{k_{\text {off }}}=\frac{[A][B]}{[A B]}
$$

The fractional saturation, or occupancy, $Y$, is the fraction of protein bound to ligand,

$$
\begin{aligned}
& Y=\frac{[A B]}{[A]+[A B]} \\
& Y=\frac{K_{\mathrm{eq}}[B]}{1+K_{\mathrm{eq}}[B]} \\
& Y=\frac{[B]}{K_{D}+[B]} \\
& Y=\frac{[A]}{K_{D}+[A]}
\end{aligned}
$$

If there are multiple reactions between a ligand, $A$, and a protein, $B_{0}$, having identical binding sites, then the intermediate states, $A B_{j}$, for $j=0, \ldots, n-1$, can be written as,

$$
\begin{gathered}
A+B_{0} \underset{k_{\mathrm{off}, 0}}{\stackrel{k_{\mathrm{on}, 0}}{\rightleftharpoons}} A B_{0}=B_{1} \\
A+B_{1} \underset{k_{\mathrm{off}, 1}}{\stackrel{k_{\mathrm{on}, 1}}{\rightleftharpoons}} A B_{1}=B_{2} \\
\ldots \\
A+B_{n-1} \underset{k_{\mathrm{off}, n-1}}{\stackrel{k_{\mathrm{on}, n-1}}{\rightleftharpoons}} A B_{n-1}=B_{n}
\end{gathered}
$$

which are equivalent to the coupled equations, 


$$
\begin{gathered}
\frac{d\left[B_{1}\right]}{d t}=k_{\mathrm{on}, 0}[A]\left[B_{0}\right]-k_{\mathrm{off}, 0}\left[A B_{0}\right] \\
\frac{d\left[B_{2}\right]}{d t}=k_{\mathrm{on}, 1}[A]\left[B_{1}\right]-k_{\mathrm{off}, 1}\left[A B_{1}\right] \\
\cdots \\
\frac{d\left[B_{n}\right]}{d t}=k_{\mathrm{on}, n-1}[A]\left[B_{n-1}\right]-k_{\mathrm{off}, n-1}\left[A B_{n-1}\right]
\end{gathered}
$$

The dissociation constants are defined for $j=1, \ldots, n$ as,

$$
K_{D, j}=\frac{k_{\mathrm{on}, j-1}}{k_{\mathrm{off}, j-1}}=\frac{[A]\left[B_{j-1}\right]}{\left[B_{j}\right]}=\frac{1}{K_{A, j}}
$$

It may be difficult to measure $\left[B_{j}\right]$. A more convenient quantity is the average number, $r$, of ligand molecules bound to the protein:

$$
r=\frac{\left[B_{1}\right]+2\left[B_{2}\right]+\ldots+n\left[B_{n}\right]}{\left[B_{0}\right]+\left[B_{1}\right\}+\ldots\left[B_{n}\right]}
$$

When the expressions for $K_{A, j}$ are substituted in, Adair's equation results:

$$
r=\frac{K_{A, 1}[A]+2 K_{A, 1} K_{A, 2}[A]^{2}+\ldots+n K_{A, 1} K_{A, 2} \ldots K_{A, n}[A]^{n}}{1+K_{A, 1}[A]+K_{A, 1} K_{A, 2}[A]^{2}+\ldots+K_{A, 1} K_{A, 2} \ldots K_{A, n}[A]^{n}}
$$

Or, defining intrinsic association constants, $K_{A, j}=\frac{(n-j+1)}{j} K_{j}$,

$$
\begin{gathered}
r=\left(n K_{1}[A]+n K_{1} K_{2}[A]^{2}+\ldots+n K_{1} K_{2} \ldots K_{n}[A]^{n}\right) / \\
\left(1+n K_{1}[A]+n \frac{(n-1)}{2} K_{1} K_{2}[A]^{2}+\ldots+n \frac{(n-1)}{2} \frac{(n-2)}{3} \ldots \frac{1}{n} K_{1} K_{2} \ldots K_{n}[A]^{n}\right) \\
r=\frac{n K_{1}[A]+n K_{1} K_{2}[A]^{2}+\ldots+n K_{1} K_{2} \ldots K_{n}[A]^{n}}{1+n K_{1}[A]+n \frac{(n-1)}{2} K_{1} K_{2}[A]^{2}+\ldots+K_{1} K_{2} \ldots K_{n}[A]^{n}} \\
Y=\frac{r}{n}=\frac{K_{1}[A]+K_{1} K_{2}[A]^{2}+\ldots+K_{1} K_{2} \ldots K_{n}[A]^{n}}{1+n K_{1}[A]+n \frac{(n-1)}{2} K_{1} K_{2}[A]^{2}+\ldots+K_{1} K_{2} \ldots K_{n}[A]^{n}}
\end{gathered}
$$


For highly cooperative binding (with $n$ identical interacting binding sites where intermediate states can be neglected because $\left.k_{\mathrm{on}, j+1} \gg k_{\mathrm{on}, j}\right)$,

$$
A+n B \underset{k_{\mathrm{off}}}{\stackrel{k_{\mathrm{on}}}{\rightleftharpoons}} C
$$

From the law of mass action, the rate is defined as, $\frac{d}{d t} A=-k_{\mathrm{on}} B^{n} A$, so the corresponding rate equation for $C$ is:

$$
\begin{gathered}
\frac{d[C]}{d t}=k_{\mathrm{on}}[A][B]^{n}-k_{\mathrm{off}}[C] \\
K_{D}=\frac{k_{\mathrm{on}}}{k_{\mathrm{off}}}=\frac{[A][B]^{n}}{[C]} \\
Y=\frac{[B]^{n}}{K_{D}+[B]^{n}} \\
Y=\frac{[A]^{n}}{K_{D}+[A]^{n}}
\end{gathered}
$$




\section{Optimization procedure}

The optimization problem is defined by:

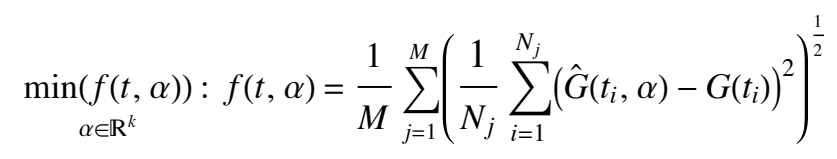

where $G$ is the data (normalized gain), $\hat{G}$ is the model, $\alpha$ is the vector of model parameters, $N_{j}$ is the number of points in the $j^{\text {th }}$ data set, and $M$ is the number of data sets included in the minimization problem. Five of the eight ordered trains and one of the two complex trains were included in the fitting set. Six of the 32 model parameters were fixed, resulting in a $k=26$ dimensional search space. The ratio of data points to parameters was 12.2. The parameters were box-constrained to physiologically reasonable values, and optionally scaled by the sensitivity of the cost function to the individual parameters around the initial point. The parameters and their constraints are listed in Table 2.3.

In addition to the error defined in Eqn.(C.1), several of the parameters were given a crude form of regularization in which a linearly increasing error was added if the parameters exceeded a connected set within their bounded domain. Also, certain data points were weighted, including the PPF points in the ordered trains, the depressed points in the ordered trains, and the two extreme points of the dynamic range in all data sets. These selected data points were given weight values between 5 and 45 . Finally, certain physiologically motivated constraints were established for some parameters. For instance, the initial calcium-dependent probabilities of syt-SNARE binding were constrained to be less than the maximal calcium-saturated probabilities.

Sequential quadratic programming (SQP) was initially used to solve the optimization problem. The Matlab function, fmincon, was used to implement the optimization routine. Gradients were approximated 
numerically by forward difference. The quadratic programming subproblems were solved using the active set method. The function fmincon was modified to allow for vectorization where possible, resulting in at least a 5x speedup. Also, the DSCI model function evaluations made use of graphics processing units (GPUs) through the Accelereyes Jacket interface. The performance of the GPUs exceeded the CPUs when at least 15,000 DSCI models were simultaneously evaluated, but memory resources limited the number of model evaluations to about 200,000 . At the upper limit of function evaluations, the GPUs provided about a 4x speedup over CPU performance. GPUs were used to evaluate the DSCI model with a large batch of randomly sampled parameters. From this set, the best 1,000 parameter vectors were applied to the optimization problem.

To identify local minima, the resulting parameter vectors were hierarchically clustered using the Ward linkage method. Prior to clustering, parameters were normalized and sorted according to the ratio of the standard deviation to the kurtosis in their distributions. This statistic estimated the tendency of the parameters to cluster, allowing the parameters that did not vary to be excluded from the clustering routine, thereby improving the clustering results. Local minima were identified based on a threshold value in the difference between the distances between the clusters. The cross-correlogram of the clustered parameters was used to visualize the minima.

The identification of local minima in the parameter space allowed parameters to be automatically selected for initializing subsequent rounds of optimization. Local minima could be thoroughly explored through multiple iterations of this process, and occasionally new minima discovered. However, given the tendency of the SQP algorithm to find local minima, as well as the relative innefficiency of the SQP algorithm (explainable mostly by the extra processes required to calculate numerical gradients), the vast parameter space was still under-explored. The performance of the SQP algorithm on the problem was not explainable by non-differentiability of the error surface. 
To improve the search efficiency, the derivative-free Nelder-Mead method of optimization was employed. The Nelder-Mead method was prone to early convergence (presumably to non-stationary points), but with restarting, the Nelder-Mead algorithm operated about 10 times more efficiently than SQP. However, despite the improvement in efficiency, the Nelder-Mead method, like the SWP method, did not take full advantage of the GPUs (because of the relatively small number of models that could be run for any appreciable number of iterations), so in an effort to increase the use of the GPUs, another direct, derivative-free search strategy was developed.

In the new search strategy, dubbed here the shell-search method, parameters were randomly selected at a fixed radius from the initial point, as defined by some fraction of the bounded domain. If, in the sampling of this shell around the initial point, a value lower than the initial point was found, a line search was performed in the direction of the lowest value, while respecting the parameter bounds. The next iteration was centered on the new point, with a slightly smaller shell radius (the radius was decreased by a small, fixed fraction). If a lower point was not found, the shell radius was increased by a small fixed fraction, and the search repeated until either a lower point was found, or some maximum radius limit achieved. If, after a fixed number of iterations, no change in the current lowest error value was recorded, the shell was reset to the initial radius, and the process repeated, until some stopping criteria was met (for instance, number of iterations or negligable change in error).

Finally, to insure that local minima were achieved, a direct search method was applied in one of two strategies. In one case, each of the model parameters was first varied by a fixed fraction of it's bounded domain, and the parameter that generated the largest decrease in the error was adjusted to the new value. In the other case, each parameter was simply varied in turn, so that the parameters were cycled through sequentially. When no further improvements were made at the current parameter variation step, the fraction of parameter variation was decreased by a determined amount. Again, the process was terminated when some stopping criteria was met. 
The mean performance of the optimization methods are compared in Fig.C.1 $(n=4)$. In the figures, an equivalent evaluation time is compared. This way, the efficiency of the methods can be compared. The initial point and the variation around the initial point were controlled in these tests, but the starting points were randomly selected at the start of each test. Each of the four comparisons started with 100 randomly initialized points. The Nelder-Mead method, while the most efficient, did not always reach the lowest error. The surprising result of this comparison is that one of the direct search methods outperformed the SQP method on this problem.

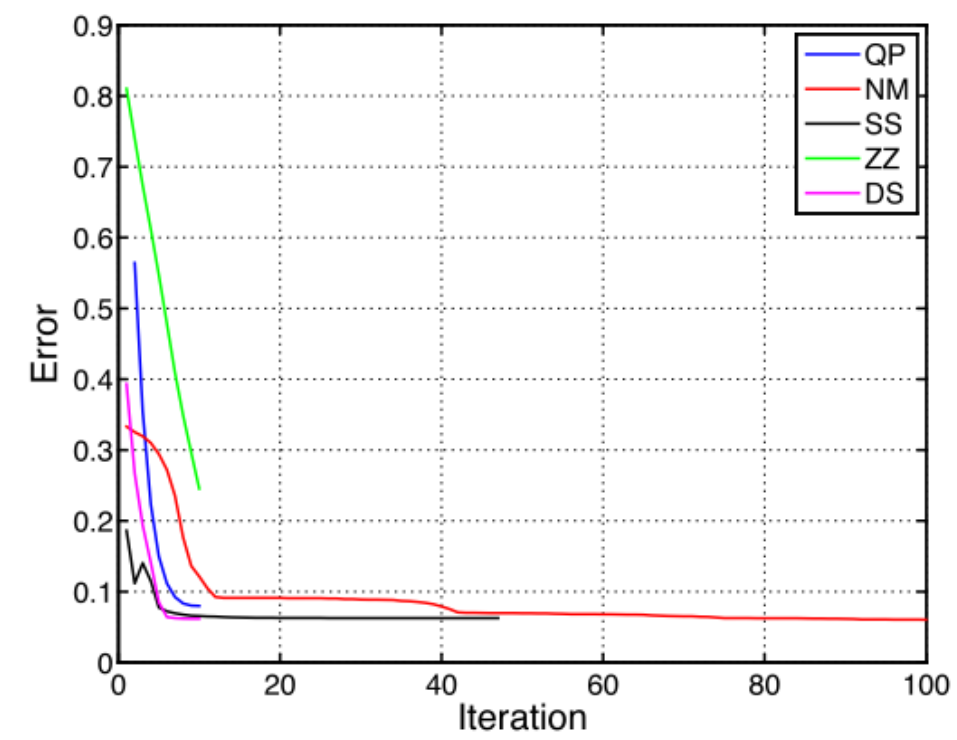

Figure C.1. Comparison of five search methods. The mean of four trials of each method are shown for an equivalent time. Shown are means of the searches from each of the four trials that finished with the lowest value. (QP = quadratic programming, $\mathrm{NM}=$ Nelder-Mead, $\mathrm{SS}=$ shell search, $\mathrm{ZZ}=$ zig-zag, DS = direct search). All optimization was performed in Matlab. 


\section{A P P E N D I X D}

\section{CA1 pyramidal cell model}

\section{The Pinsky-Rinzel CA3 model}

The CA1 pyramidal cell used in the simulations is based on a CA3 pyramidal model published in 1994 [Pinsky and Rinzel, 1994]. The Pinsky-Rinzel model is a two-compartment reduction of a previously developed 19-compartment model [Traub et al, 1991]. One of the compartments in the Pinsky-Rinzel model describes the soma of the cell, and the other compartment describes the dendrites. Both compartments are assumed to have a spherical shape.

The model is presented below as published by Pinsky and Rinzel, however, the model was modified to simulate CA1 pyramidal cell behavior. CA1 pyramidal cells display more regular spiking behavior (less bursting) than CA3 pyramidal cells, due to a reduced calcium conductance in the dendrite. The modifications also include an additional leak conductance with a potassium reversal potential, and an adjustment of the coupling parameters between the compartments.

The somatic compartment voltage of the CA3 model is defined by,

$$
C_{M} \frac{d V_{s}}{d t}=-I_{\text {leak }}\left(V_{s}\right)-I_{\mathrm{Na}}\left(V_{s}, h\right)-I_{K-\mathrm{DR}}\left(V_{s}, n\right)+\frac{g_{c}}{p}\left(V_{d}-V_{s}\right)+\frac{I_{s}}{p}
$$

The parameters $p$ and $g_{c}$, are a coupling constants between the two compartments. The individual currents are described by,

$$
\begin{gathered}
I_{\text {leak }}\left(V_{s}\right)=\bar{g}_{\text {leak }}\left(V_{s}-V_{\text {leak }}\right) \\
I_{\mathrm{Na}}\left(V_{s}, h\right)=\bar{g}_{\mathrm{Na}} m_{\infty}^{2}\left(V_{s}\right) h\left(V_{s}-V_{\mathrm{Na}}\right) \\
I_{K-\mathrm{DR}}\left(V_{s}, n\right)=\bar{g}_{K-\mathrm{DR}} n\left(V_{s}-V_{K}\right),
\end{gathered}
$$

where, 


$$
\begin{gathered}
\frac{d h}{d t}=\frac{h_{\infty}\left(V_{s}\right)-h}{\tau_{h}\left(V_{s}\right)} \\
\frac{d n}{d t}=\frac{n_{\infty}\left(V_{s}\right)-n}{\tau_{n}\left(V_{s}\right)},
\end{gathered}
$$

with,

$$
\begin{aligned}
& m_{\infty}\left(V_{s}\right)=\frac{\alpha_{m}\left(V_{s}\right)}{\alpha_{m}\left(V_{s}\right)+\beta_{m}\left(V_{s}\right)} \\
& h_{\infty}\left(V_{s}\right)=\frac{\alpha_{h}\left(V_{s}\right)}{\alpha_{h}\left(V_{s}\right)+\beta_{h}\left(V_{s}\right)} \\
& \tau_{h}\left(V_{s}\right)=\frac{1}{\alpha_{h}\left(V_{s}\right)+\beta_{h}\left(V_{s}\right)} \\
& n_{\infty}\left(V_{s}\right)=\frac{\alpha_{n}\left(V_{s}\right)}{\alpha_{n}\left(V_{s}\right)+\beta_{n}\left(V_{s}\right)} \\
& \tau_{n}\left(V_{s}\right)=\frac{1}{\alpha_{n}\left(V_{s}\right)+\beta_{n}\left(V_{s}\right)},
\end{aligned}
$$

and,

$$
\begin{gathered}
\alpha_{m}\left(V_{s}\right)=\frac{0.32\left(13.1-V_{s}\right)}{e^{\frac{13.1-V_{s}}{4}}-1} \\
\beta_{m}\left(V_{s}\right)=\frac{0.28\left(V_{s}-40.1\right)}{e^{\frac{40.1-V_{s}}{5}}-1} \\
\alpha_{h}\left(V_{s}\right)=0.128 e^{\frac{17-V_{s}}{18}} \\
\beta_{h}\left(V_{s}\right)=\frac{4}{1+e^{\frac{40-V_{s}}{5}}} \\
\alpha_{n}\left(V_{s}\right)=\frac{0.016\left(35.1-V_{s}\right)}{e^{\frac{35.1-V_{s}}{5}}-1} \\
\beta_{n}\left(V_{s}\right)=0.25 e^{0.5-0.025 V_{s}} .
\end{gathered}
$$

The dendritic compartment voltage of the CA 3 model is defined by, 


$$
\begin{gathered}
C_{M} \frac{d V_{d}}{d t}= \\
-I_{\text {leak }}\left(V_{d}\right)-I_{\mathrm{Ca}}\left(V_{d}, s\right)-I_{K-\mathrm{AHP}}\left(V_{d}, q\right)-I_{K-C}\left(V_{d}, \mathrm{Ca}, c\right)-\frac{I_{\mathrm{syn}}}{1-p}+\frac{g_{c}}{1-p}\left(V_{s}-V_{d}\right)+\frac{I_{d}}{1-p},
\end{gathered}
$$

where the currents are described by,

$$
\begin{gathered}
I_{\text {leak }}\left(V_{d}\right)=\bar{g}_{\text {leak }}\left(V_{d}-V_{\text {leak }}\right) \\
I_{\mathrm{Ca}}\left(V_{d}, s\right)=\bar{g}_{\mathrm{Ca}} s^{2}\left(V_{d}-V_{\mathrm{Ca}}\right) \\
I_{K-\mathrm{AHP}}\left(V_{d}, q\right)=\bar{g}_{K-\mathrm{AHP}} q\left(V_{d}-V_{K}\right) \\
I_{K-C}\left(V_{d}, \mathrm{Ca}, c\right)=\bar{g}_{K-C} c \chi(\mathrm{Ca})\left(V_{d}-V_{K}\right),
\end{gathered}
$$

with,

$$
\begin{gathered}
\frac{d s}{d t}=\frac{s_{\infty}\left(V_{d}\right)-s}{\tau_{s}\left(V_{d}\right)} \\
\frac{d q}{d t}=\frac{q_{\infty}\left(V_{d}\right)-q}{\tau_{q}\left(V_{d}\right)} \\
\frac{d c}{d t}=\frac{c_{\infty}\left(V_{d}\right)-c}{\tau_{c}\left(V_{d}\right)} \\
\frac{d \mathrm{Ca}}{d t}=-0.13 I_{\mathrm{Ca}}-0.075 \mathrm{Ca},
\end{gathered}
$$

and,

$$
\begin{gathered}
\alpha_{s}\left(V_{d}\right)=\frac{1.6}{1+e^{-0.072\left(V_{d}-65\right)}} \\
\beta_{s}\left(V_{d}\right)=\frac{0.02\left(V_{d}-51.1\right)}{e^{\frac{V_{d}-51.1}{5}}-1} \\
\alpha_{q}(\mathrm{Ca})=\min (0.00002 \mathrm{Ca}, 0.01) \\
\beta_{q}=0.001 \\
\alpha_{c}\left(V_{d}\right)=\frac{e^{\frac{V_{d}-10}{11}}-e^{\frac{V_{d}-6.5}{27}}}{18.975}, \text { for } V_{d} \leq 50,
\end{gathered}
$$




$$
\begin{gathered}
\alpha_{c}\left(V_{d}\right)=2 e^{\frac{6.5-V_{d}}{27}}, \text { for } V_{d}>50 \\
\beta_{c}\left(V_{d}\right)=2 e^{\frac{6.5-V_{d}}{27}}-\alpha_{c}, \text { for } V_{d} \leq 50 \\
\beta_{c}\left(V_{d}\right)=0, \text { for } V_{d}>50 \\
\chi(\mathrm{Ca})=\min \left(\frac{\mathrm{Ca}}{250}, 1\right) .
\end{gathered}
$$

The synaptic currents are described by,

$$
I_{\mathrm{syn}}=I_{\mathrm{AMPA}}+I_{\mathrm{NMDA}},
$$

where,

$$
I_{\mathrm{AMPA}}\left(V_{d}, V_{\mathrm{syn}}\right)=\bar{g}_{\mathrm{AMPA}} W_{i}(t)\left(V_{d}-V_{\mathrm{syn}}\right),
$$

with,

$$
\frac{d W_{i}}{d t}=\sum_{j=1}^{N} \Theta\left(V_{s, j}-20\right)-\frac{W_{i}}{2},
$$

and,

$$
I_{\mathrm{NMDA}}\left(V_{d}, V_{\mathrm{syn}}\right)=\bar{g}_{\mathrm{NMDA}} S_{i}(t) \frac{\left(V_{d}-V_{\mathrm{syn}}\right)}{1+0.28 e^{-0.062\left(V_{d}-60\right)}}
$$

with,

$$
\frac{d S_{i}}{d t}=\sum_{j=1}^{N} \Theta\left(V_{s, j}-10\right)-\frac{S_{i}}{150} .
$$

In these expressions, $\Theta(\cdot)$ is the Heaviside function, and $N$ is the total number of cells synapsing onto the $i^{\text {th }}$ cell.

\section{Parameter values published in [Pinsky and Rinzel, 1994]}

Maximal conductances $\left(\frac{\mathrm{mS}}{\mathrm{cm}^{2}}\right)$ :

$$
\bar{g}_{\text {leak }}=0.1, \bar{g}_{\mathrm{Na}}=30, \bar{g}_{K-\mathrm{DR}}=15, \bar{g}_{\mathrm{Ca}}=10, \bar{g}_{K-\mathrm{AHP}}=0.8, \bar{g}_{K-C}=15 \text {. }
$$

Reversal potentials (mV): 


$$
V_{\text {leak }}=0, V_{\mathrm{Na}}=120, V_{K}=-15, V_{\mathrm{Ca}}=140, V_{\text {syn }}=60 \text {. }
$$

Coupling parameters:

$$
g_{c}=2.1 \frac{\mathrm{mS}}{\mathrm{cm}^{2}}, p=0.5
$$

The membrane capacitance, $C_{M}$, was set to $3 \frac{\mu \mathrm{F}}{\mathrm{cm}^{2}}$, and the total membrane area was set to one (which is why it doesn't appear in the equations above). The bursting behavior was illustrated in [Pinsky and Rinzel, 1994] with an applied somatic current, $I_{s}$, of $0.75 \frac{\mu \mathrm{A}}{\mathrm{cm}^{2}}$, and with an applied dendritic curent, $I_{d}$, of zero. The synaptic conductances, $\bar{g}_{\mathrm{AMPA}}$ and $\bar{g}_{\mathrm{NMDA}}$, were both set to $0 \frac{\mathrm{mS}}{\mathrm{cm}^{2}}$. The published bursting behavior is shown in Fig. D.1. The published spiking behavior for 1.5 seconds is shown in Fig. D.2.

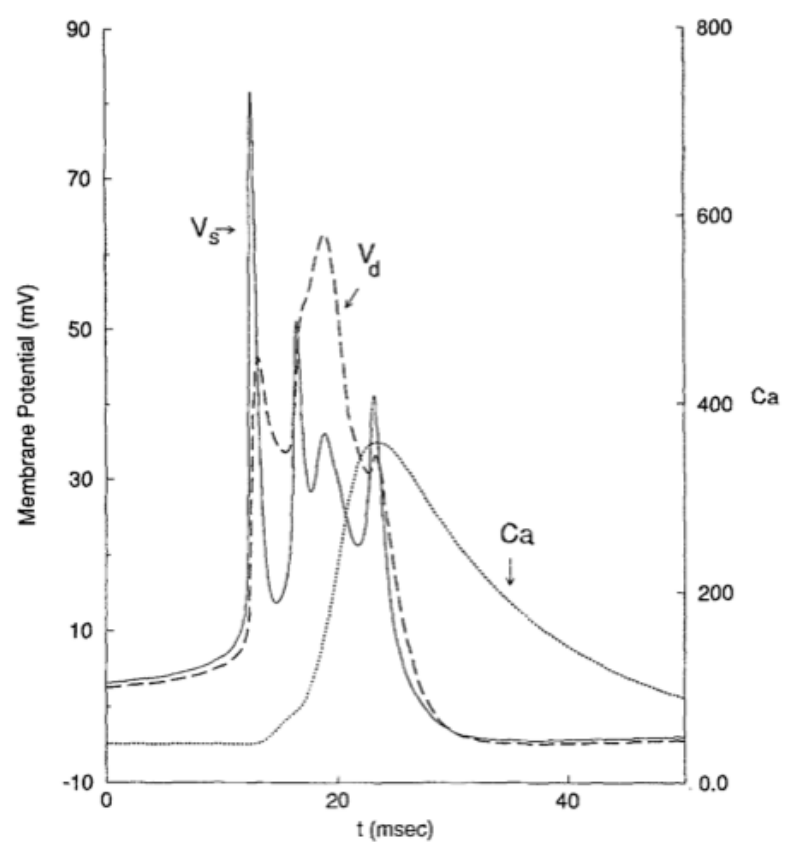

Figure D.1. Published behavior of Pinsky-Rinzel CA3 pyramidal cell spike. $V_{s}$ : somatic potential, $V_{d}$ : dendritic potential, Ca: calcium concentration. Figure credit: [Pinsky and Rinzel, 1994] 


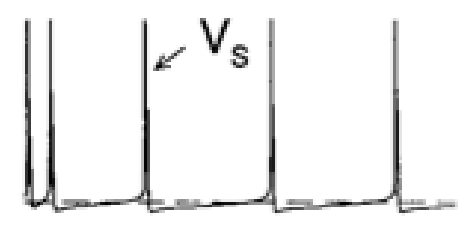

Figure D.2. Published behavior of Pinsky-Rinzel CA3 pyramidal cell for 1.5 seconds with same parameters and inputs as shown in Fig. D.1. Figure credit: [Pinsky and Rinzel, 1994]

\section{Parameter values used to recover $\mathrm{CA3}$ behavior}

To match the published behavior of the Pinsky-Rinzel CA3 model, a potassium leak current was introduced, $\bar{g}_{K \text {,leak }}=0.005 \frac{\mathrm{mS}}{\mathrm{cm}^{2}}$, with $V_{K \text {,leak }}=-15 \mathrm{mV}$, and the coupling parameters were adjusted to, $g_{c}=1.625 \frac{\mathrm{mS}}{\mathrm{cm}^{2}}$, and $p=0.325$. The other parameters were unchanged. The model was integrated implicitly using the Crank-Nicolson method with a constant time step of $0.05 \mathrm{~ms}$. The linear system solved at each time step using the method described in (Gaussian elimination) [Dayan and Abbott, 2001]. The model behavior is shown in Figs. D.3 and D.4.

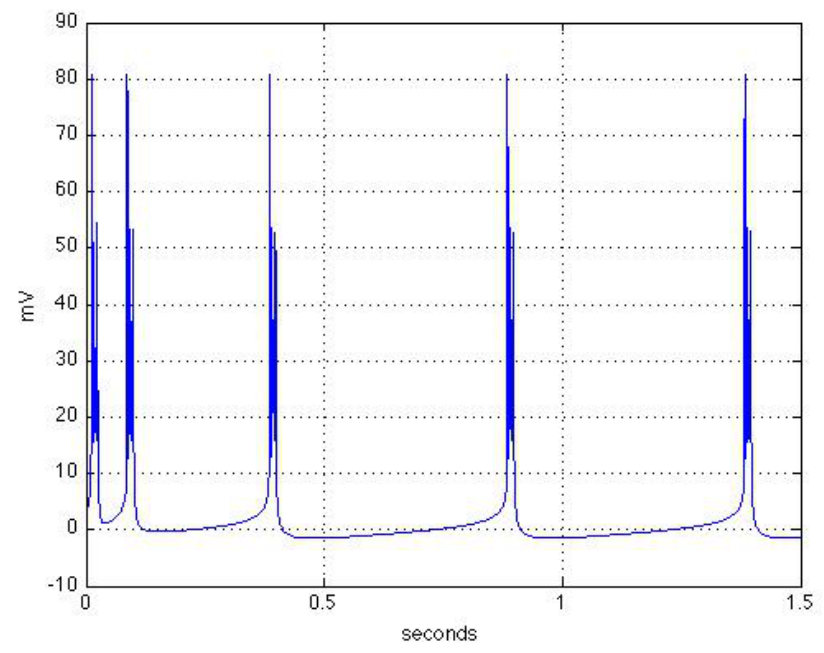

Figure D.3. Behavior of modified Pinsky-Rinzel CA3 pyramidal cell for 1.5 seconds. Compare to Fig. D.2. 


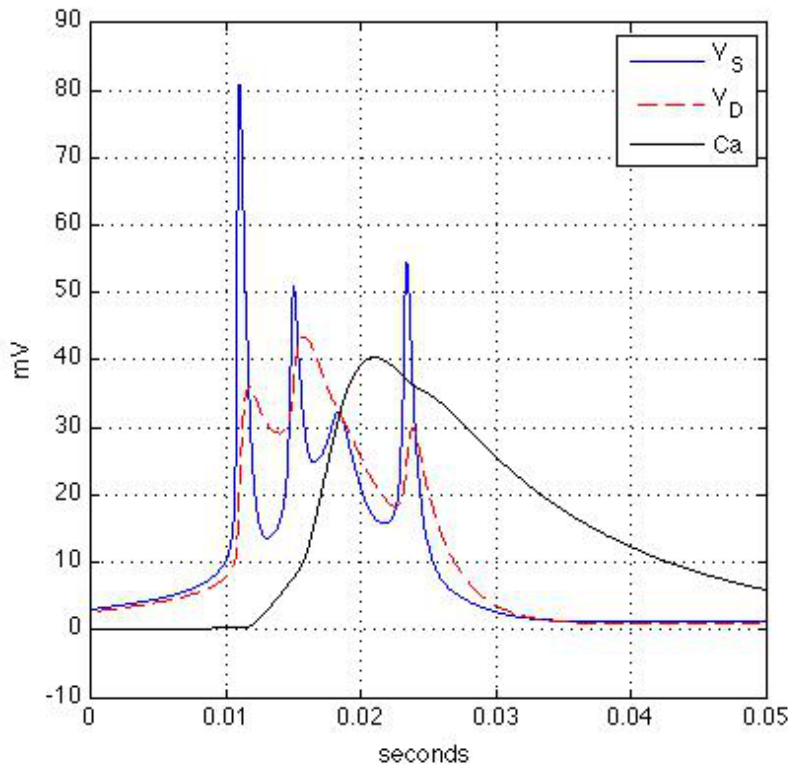

Figure D.4. Behavior of modified Pinsky-Rinzel CA3 pyramidal cell spike. Compare to Fig. D.1. Calcium is displayed on a different scale.

\section{Simulating CA1 behavior}

To obtain CA1 pyramidal cell behavior, the maximum calcium conductance, $\bar{g}_{\mathrm{Ca}}$, was reduced from 10 to $3.5 \frac{\mathrm{mS}}{\mathrm{cm}^{2}}$. This reduces the prolonged depolarizing effect of the slow calcium channel kinetics. The model behavior is compared to another CA1 model that was also based upon the Pinsky-Rinzel CA3 model [Ferguson, KA and Campbell, SA (unpublished), "A two-compartment model of a CA1 pyramidal neuron"]. The Ferguson and Campbell model added an inward calcium current, an outward potassium after-hyperpolarization current, and an outward calcium-activated potassium current to the soma compartment. For validation, a somatic current of $1.25 \frac{\mu \mathrm{A}}{\mathrm{cm}^{2}}$ was applied. The Ferguson and Campbell response to the injected somatic current is shown in Fig. D.5. The behavior of the CA1 model used in this dissertation in response to the injected current is shown in Fig. D.6. 


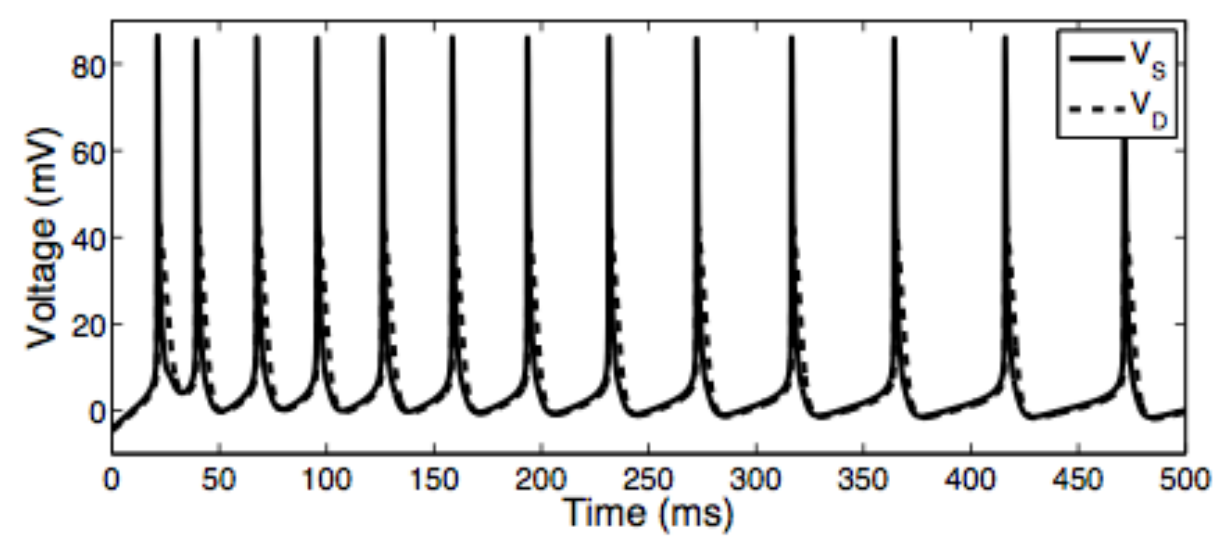

Figure D.5. Behavior of Ferguson and Campbell CA1 model in response to injected current of 1.25 $\frac{\mu \mathrm{A}}{\mathrm{cm}^{2}}$ applied to the soma. Compare to Fig. D.6. Figure credit: [Ferguson and Campbell]

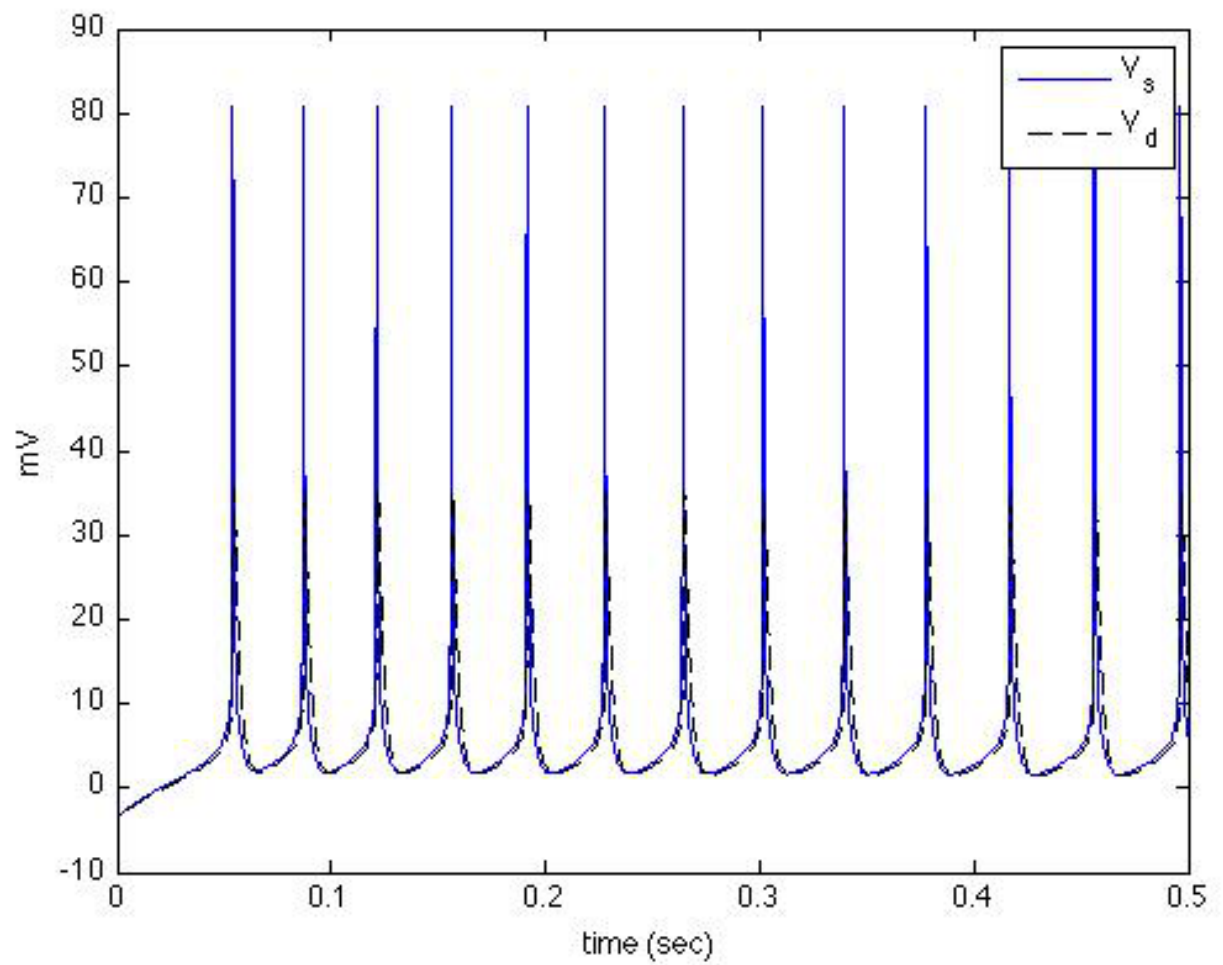

Figure D.6. Behavior of the CA1 model used in this dissertation in response to injected current of $1.25 \frac{\mu \mathrm{A}}{\mathrm{cm}^{2}}$ applied to the soma. 
In the simulations involving synaptic inputs, both of the applied currents, $I_{s}$ and $I_{d}$, were set to zero, and the synaptic conductances, $\bar{g}_{\mathrm{AMPA}}$ and $\bar{g}_{\mathrm{NMDA}}$, were both set to $0.0015 \frac{\mathrm{mS}}{\mathrm{cm}^{2}}$.

\section{Numerical integration}

The CA1 model was integrated numerically using the Crank-Nicolson scheme with a fixed time step of $0.05 \mathrm{~ms}$. The Crank-Nicolson scheme is a semi-implicit numerical integration method that uses a central-difference approximation to the time derivative at time $t=t_{i}+\frac{1}{2} \Delta t$. For an ordinary differential equation with $n$ unknowns in the vector $\phi$,

$$
\frac{d \phi(t)}{d t}=f(\phi(t), t)
$$

the Crank-Nicolson method approximates the solution to $\phi$ at time $t_{i+1}$ by,

$$
\phi\left(t_{i+1}\right)=\phi\left(t_{i}\right)+\frac{1}{2} \Delta \mathrm{t}\left[f\left(\phi\left(t_{i}\right), t_{i}\right)+f\left(\phi\left(t_{i+1}\right)\right), t_{i+1}\right] .
$$

The method is implicit because the solution, $\phi\left(t_{i+1}\right)$ appears on both sides of the equation.

The Crank-Nicolson scheme exhibits numerical stability and has error of $O(\Delta t)^{3}$, but is computationally intensive because a system of algebraic equations must be solved at each time step. The literature on compartmental modeling of neural systems refers to an efficient method of solving Eqn. (D.42) [Dayan and Abbott, 2001].

The code to numerically integrate the CA1 model was implemented in Matlab.

\section{Stochastic background current}

The stochastic background current fluctuation defined by Destexhe et al, consists of excitatory and inhibitory conductances, $g_{e}(t)$ and $g_{i}(t)$,

$$
I_{\mathrm{syn}}=g_{e}(t)\left(V-E_{e}\right)+g_{i}(t)\left(V-E_{i}\right),
$$

where the conductances are defined by a stochastic process [Uhlenbeck and Ornstein, 1930], 


$$
\begin{gathered}
\frac{d g_{e}(t)}{d t}=-\frac{1}{\tau_{e}}\left[g_{e}(t)-g_{\mathrm{e} 0}\right]+\sqrt{D_{e}} \chi_{1}(t) \\
\frac{d g_{i}(t)}{d t}=-\frac{1}{\tau_{i}}\left[g_{i}(t)-g_{\mathrm{i} 0}\right]+\sqrt{D_{i}} \chi_{2}(t),
\end{gathered}
$$

where the $\tau$ are time constants, $g_{\mathrm{e} 0}$ and $g_{\mathrm{i} 0}$ are average conductances, $D_{e}$ and $D_{i}$ are noise diffusion coefficients, and the $\chi$ are Gaussian white noise with zero mean and unit standard deviation. The numerical scheme for these equations is [Gillespie, 1996]:

$$
\begin{aligned}
& g_{e}(t+\Delta \mathrm{t})=g_{\mathrm{e} 0}+\left[g_{e}(t)-g_{\mathrm{e} 0}\right] e^{-\Delta \mathrm{t} / \tau_{e}}+A_{e} N_{1}(0,1) \\
& g_{i}(t+\Delta \mathrm{t})=g_{\mathrm{i} 0}+\left[g_{i}(t)-g_{\mathrm{i} 0}\right] e^{-\Delta \mathrm{t} / \tau_{i}}+A_{i} N_{2}(0,1),
\end{aligned}
$$

where $N_{1}(0,1)$ and $N_{2}(0,1)$ are normal random numbers, and $A_{e}$ and $A_{i}$ are amplitudes given by,

$$
\begin{gathered}
A_{e}=\sqrt{\frac{D_{e} \tau_{e}}{2}\left[1-e^{-\frac{2 \Delta t}{\tau_{e}}}\right]} \\
A_{i}=\sqrt{\frac{D_{i} \tau_{i}}{2}\left[1-e^{-\frac{2 \Delta t}{\tau_{i}}}\right] .}
\end{gathered}
$$

Values for the parameters were taken directly from the pubished vaues [Destexhe et al, 2001]. 


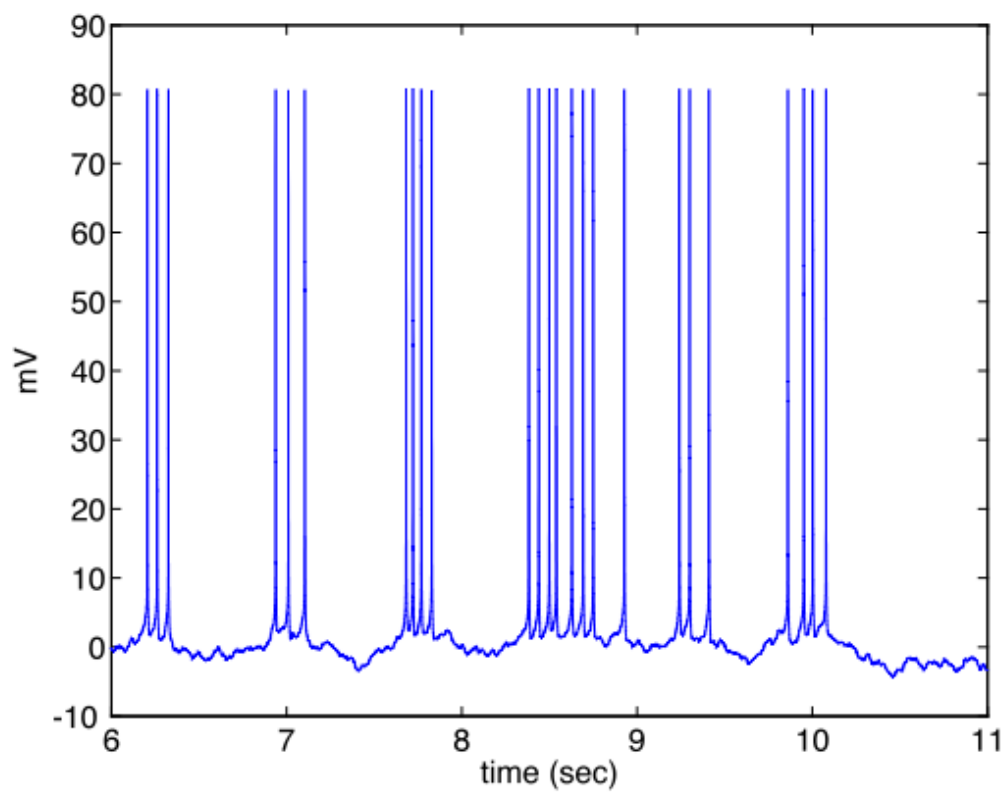

Figure D.7. Example of CA1 model spiking behavior in the presence of stochastic background current. Input consists of 75 trains sampled at $5 \mathrm{~Hz}$ mean frequency from Frerking empirical CA3 ISI distribution [Frerking et al, 2005].

\section{Input/output characterization}

The mean input/output spike rate curves for the CA1 model are shown in Fig. D.8. These curves establish the range of expected output spike rates. The input current was adjusted by increasing the number of input trains, and the tests were performed in the presence of stochastic background current fluctuations. Inputs having 25, 50, 75, and 100 constant-rate trains from $1 \mathrm{~Hz}$ to $15 \mathrm{~Hz}$ were applied, and from these tests, it was determined that 75 inputs provided the best dynamic range between $1 \mathrm{~Hz}$ and 10 Hz. Figure D.9 shows the confidence intervals on the distributions of the output rates for 10 trials of the 75-input test. 

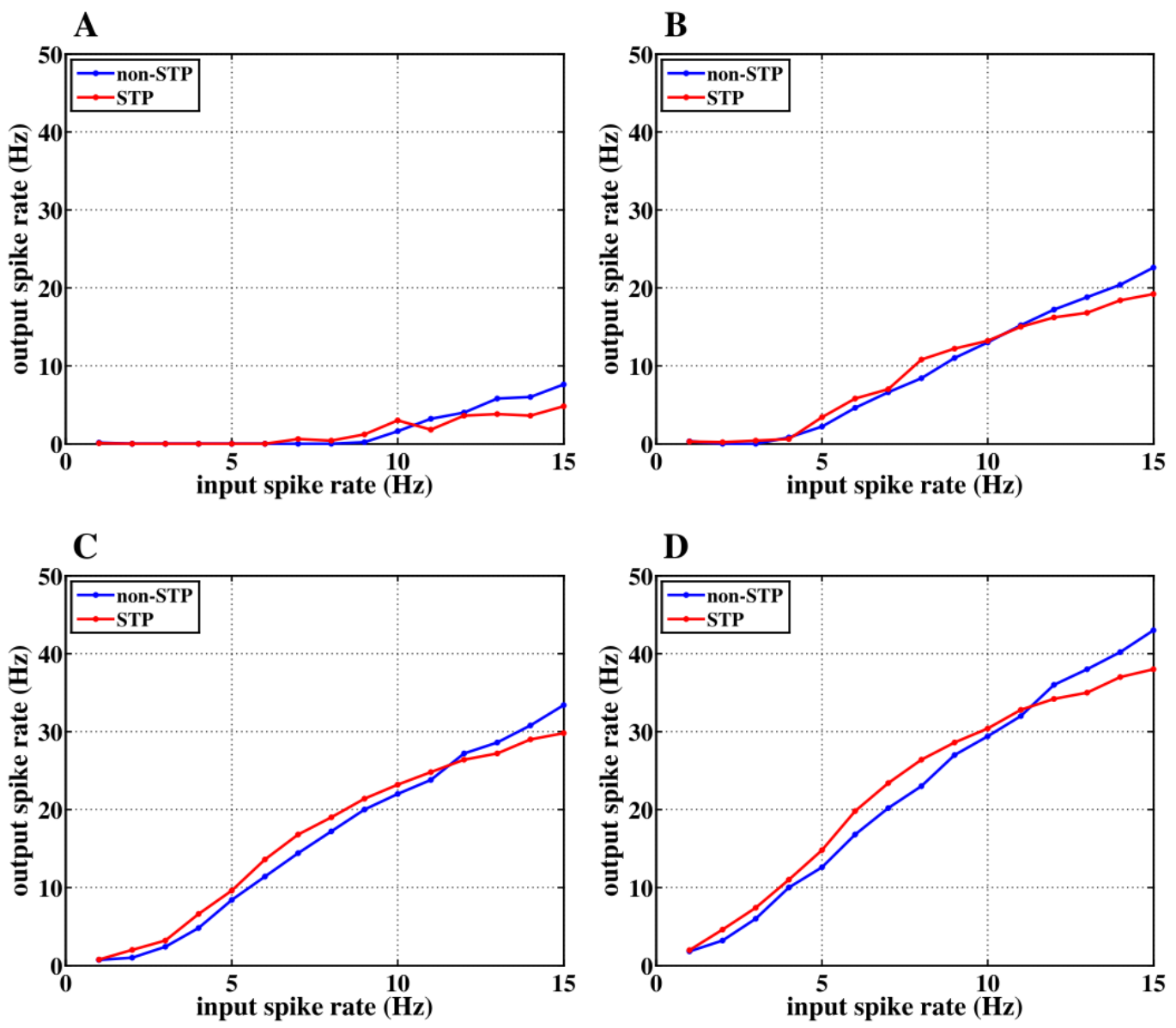

Figure D.8. CA1 pyramidal cell model input/output rate functions. (A): 25 input spike trains. (B): 50 input spike trains. (C): 75 input spike trains. (D): 100 input spike trains. Stochastic background current added. No correlation, jitter, or modulation of input trains was applied. 


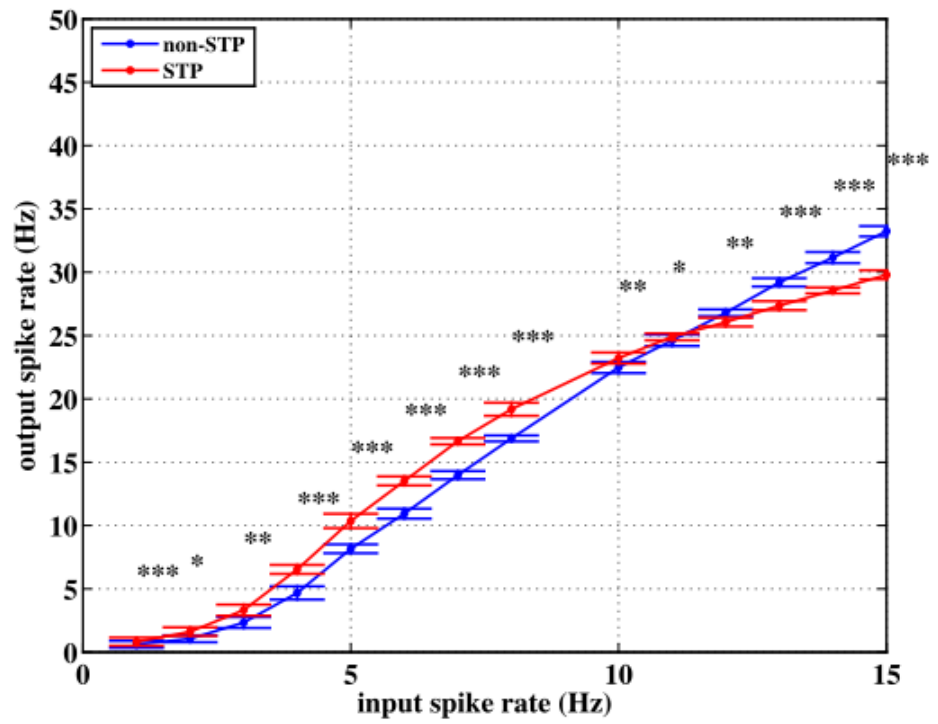

Figure D.9. CA1 pyramidal cell model input/output rate function with 75 input spike trains $(n=10)$. Stochastic background current added. No correlation, jitter, or modulation of input trains. Significance shown above plot points $(* \Rightarrow 0.01<p \leq 0.05, * * \Rightarrow 0.001<p \leq 0.01, * * * \Rightarrow p \leq 0.001)$. 